Army Environmental Quality/Installations Program

\title{
Environmental Assessment During Intelligence Preparation of the Battlefield (IPB) - Evaluation of the TIC-Master Computer Program
}

Victor F. Medina, Dennis L. Brandon, K. James Hay,

April 2012

Dhiren S. Khona, Heather K. Smith, and Agnes B. Morrow

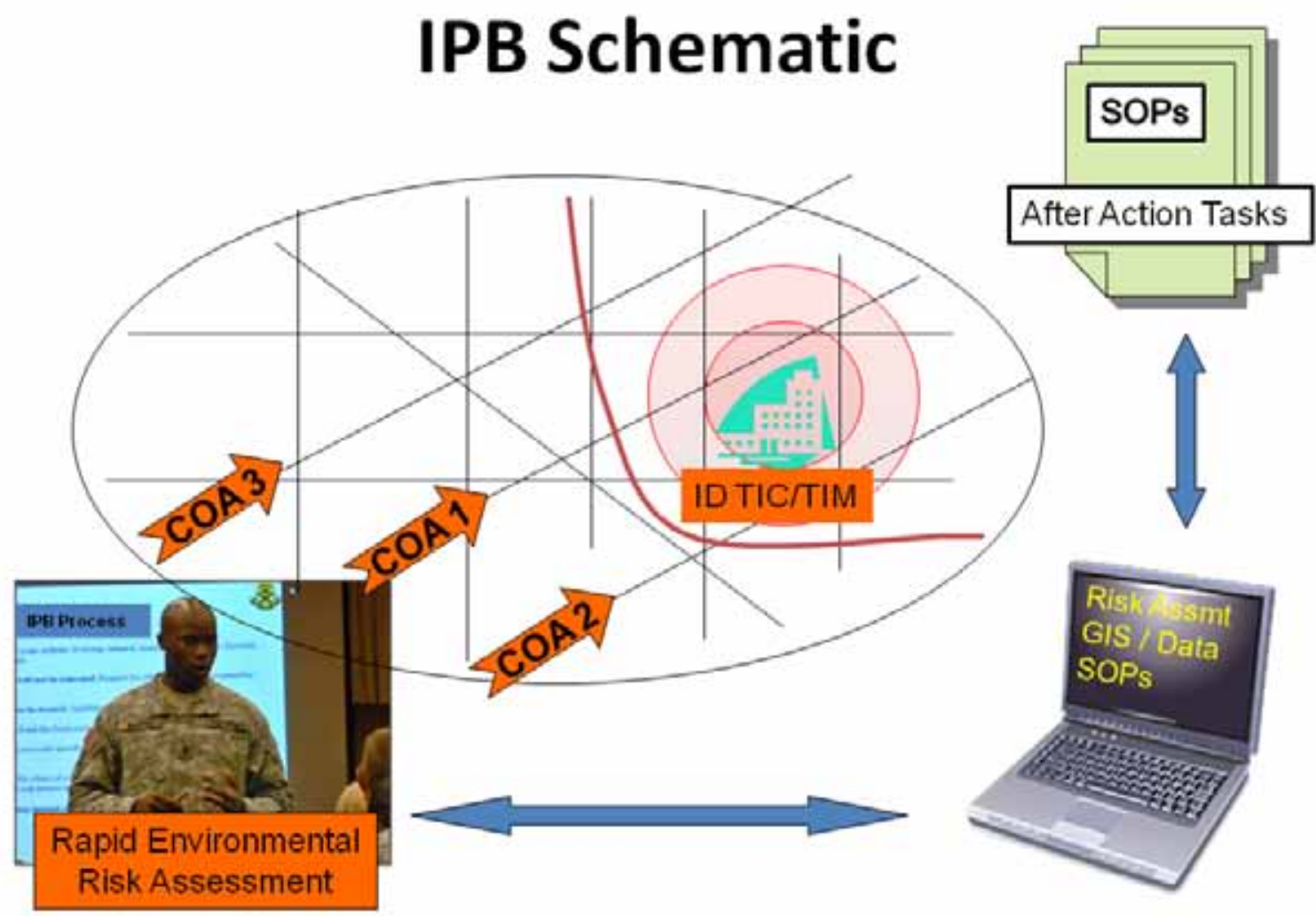




\title{
Environmental Assessment During Intelligence Preparation of the Battlefield (IPB) - Evaluation of the TIC-Master Computer Program
}

\author{
Victor F. Medina, Dennis L. Brandon, Heather K. Smith, and Agnes B. Morrow \\ U.S. Army Engineer Research and Development Center \\ Environmental Laboratory \\ 3909 Halls Ferry Road \\ Vicksburg, MS 39180-6199 \\ K. James Hay \\ Construction Engineering Research Laboratory \\ 2902 Newmark Ave. \\ Champaign, IL 61826-9005 \\ Dhiren S. Khona \\ Army Geospatial Center \\ 7701 Telegrapn Rd. \\ Alexandria, VA 22315-3864
}

Final report

Approved for public release; distribution is unlimited. 


\section{Abstract}

The Army needs to manage threats from toxic industrial chemicals (TICs) during the Intelligence Preparation of the Battlefield (IPB) Process. This work unit involved developing a database of TICs that could pose environmental/operational risk, creating an industry/chemical crosswalk, linking the chemical database to a GIS component, applying and evaluating the system in five hypothetical scenarios. The resultant program, which was christened "TIC-Master," has the capability to enhance urban theater of operations analysis to identify TIC/toxic industrial material (TIM) affiliated activities within the battlefield operational footprint and evaluate consequence management alternatives for decision support during the IPB process. TIC-Master was tested in a series of demonstrations. Facilities in the Jackson/Vicksburg MS area were used to test the program. Actual chemical data were obtained for several facilities by securing Tier 2 EPA (Environmental Protection Agency) reports for the facilities of interest. A test team was assembled to develop scenarios based on the industries represented by the facilities. TIC-Master was then used to address the scenarios. Although some deficiencies were identified, the program was successful at encompassing the chemical risk presented by the TICs found at the facilities. Plume modeling was used to assess release scenarios. Subsequent briefings of the program were conducted with potential users at the Army Maneuver Support Center of Excellence (MSCoE) and the Army Intelligency Center (AIC). Constructive suggestions were received and are presented in this document. Overall, the potential users agree that the TICMaster could be a valuable tool for the Army and other organizations. Ultimately, populating tactical decision-making tools with critical information leads to a greater knowledge of the battlefield and increases survivability.

DISCLAIMER: The contents of this report are not to be used for advertising, publication, or promotional purposes. Citation of trade names does not constitute an official endorsement or approval of the use of such commercial products. All product names and trademarks cited are the property of their respective owners. The findings of this report are not to be construed as an official Department of the Army position unless so designated by other authorized documents. 


\section{Contents}

Figures and Tables.........................................................................................................................

Preface .........................................................................................................................

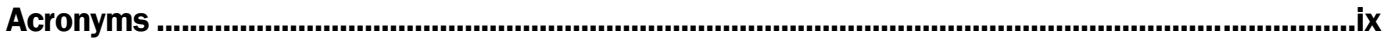

Definitions......................................................................................................................................... xii

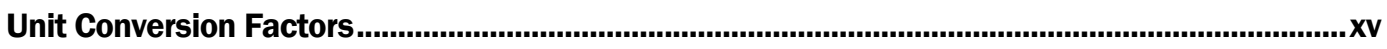

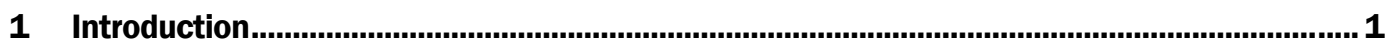

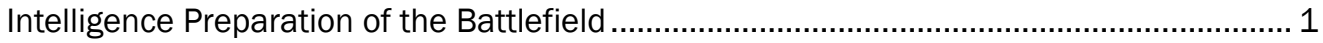

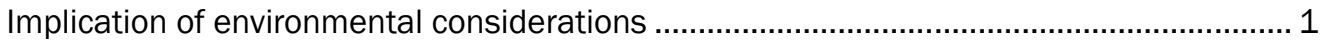

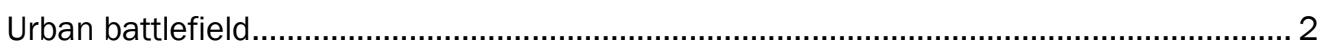

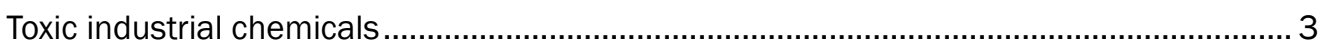

Emergency Planning and Community Right to Know Act ................................................ 5

Community Right to Know Reporting (EPA Tier II Reporting).............................................. 6

Toxic Release Inventory (TRI) reporting ..................................................................... 6

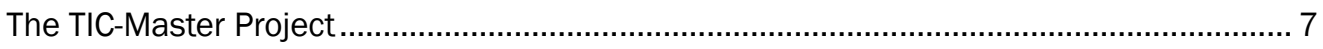

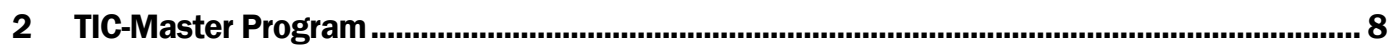

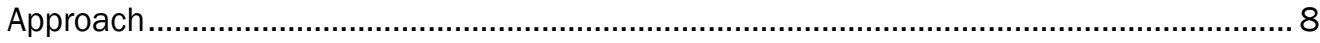

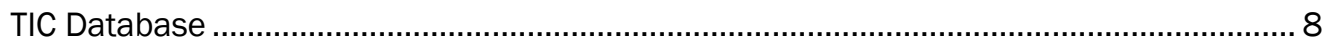

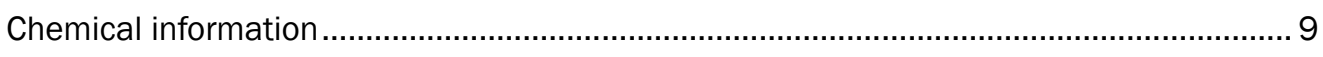

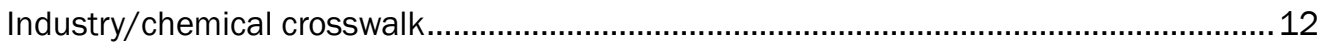

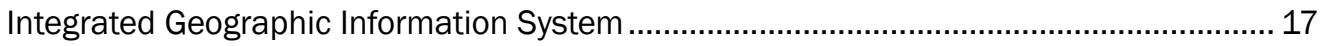

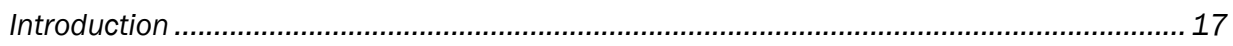

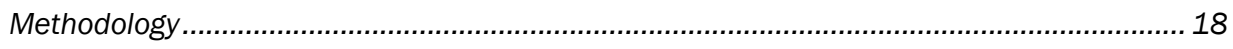

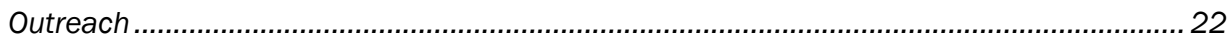

Release modeling using ALOHA (Areal Locations of Hazardous Atmospheres)...................23

Standard operating procedures for environmental releases of selected chemicals.......... 24

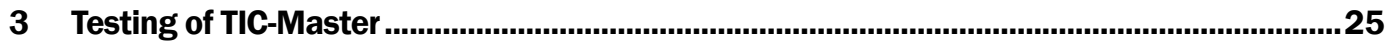

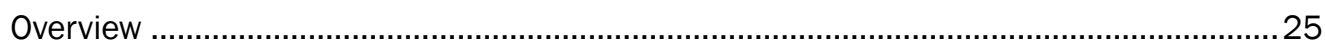

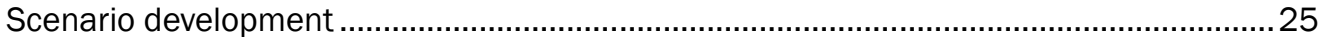

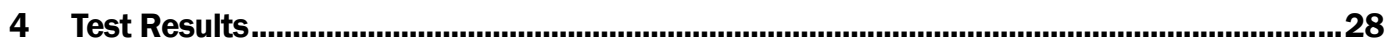

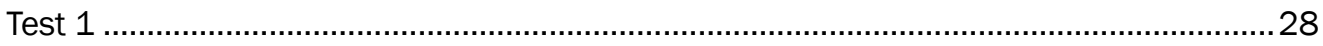

Scenario

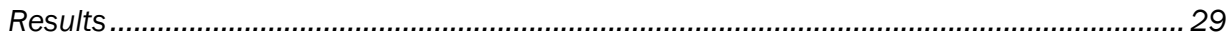

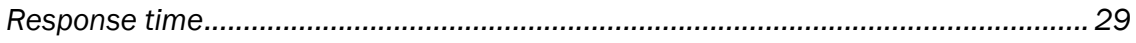

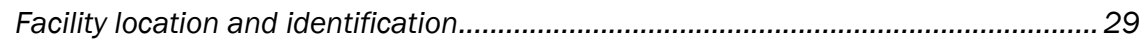

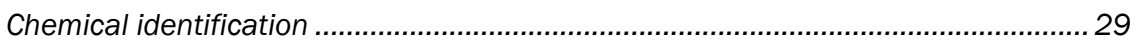

Most probable chemicals................................................................................... 33 
Most significant chemical hazard ........................................................................ 33

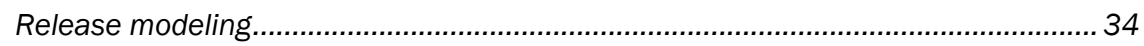

Evaluation of chemical risks from ITF-40 63 most toxic chemicals ............................. 35

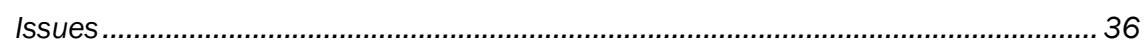

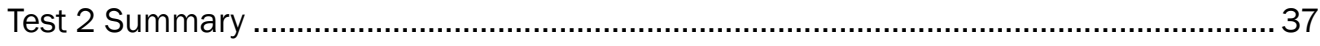

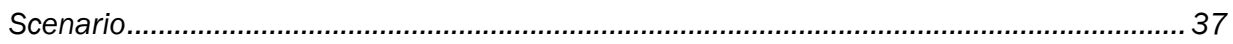

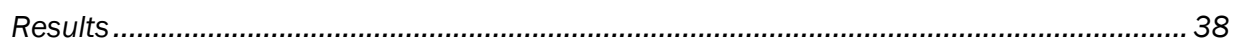

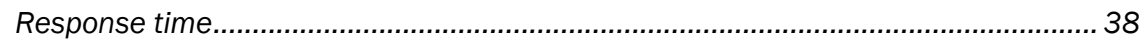

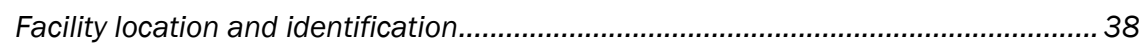

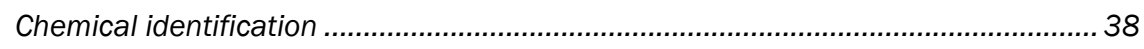

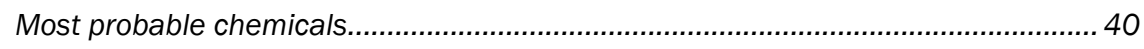

Most significant chemical hazards..................................................................... 41

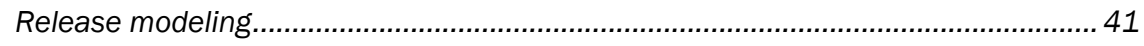

SOPs from ITF-40 63 most significant list.......................................................... 43

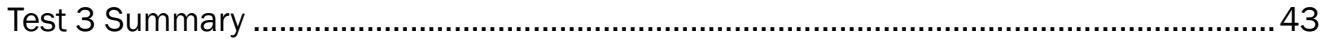

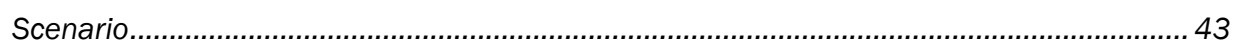

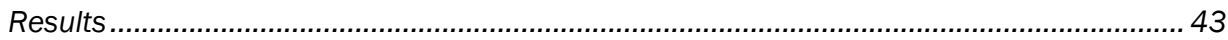

Response time

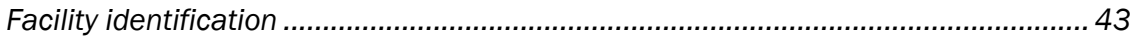

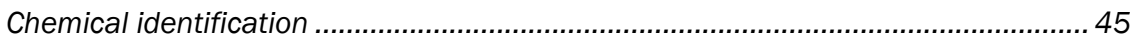

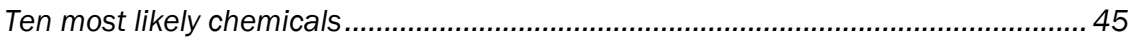

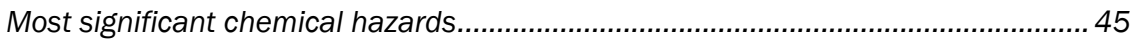

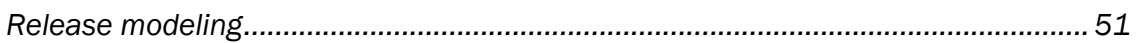

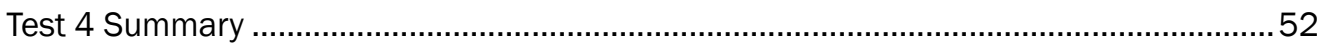

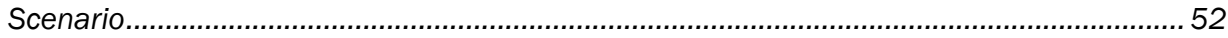

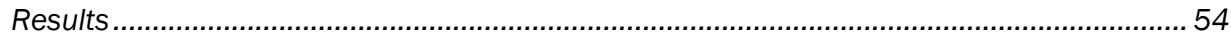

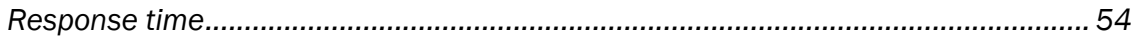

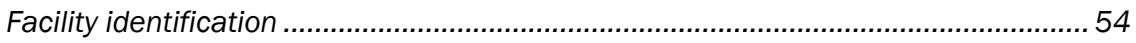

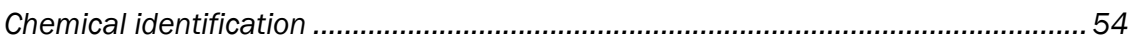

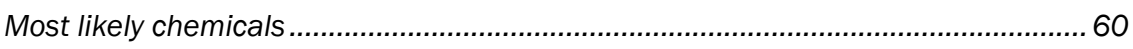

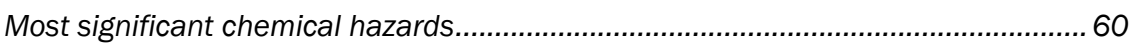

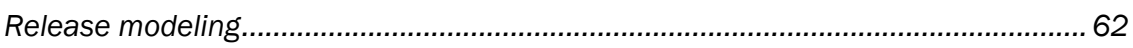

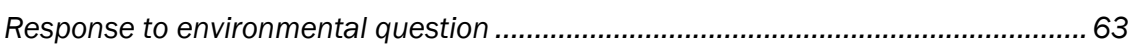

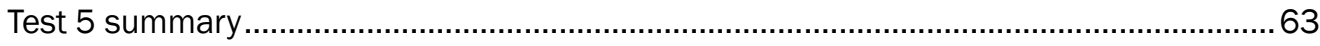

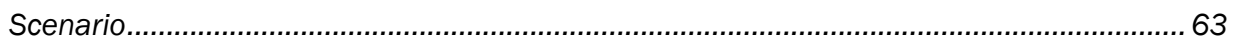

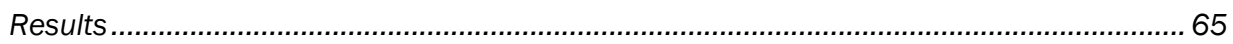

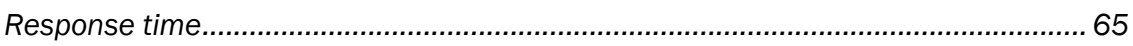

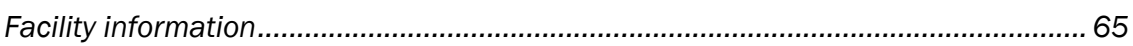

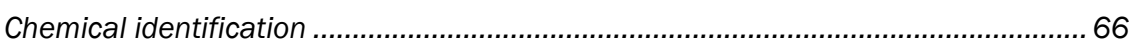

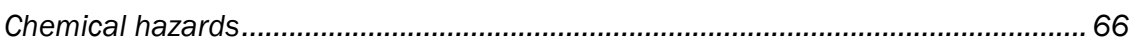

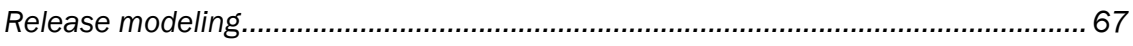

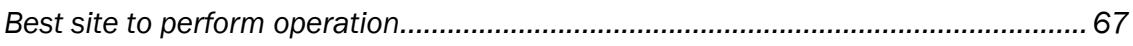

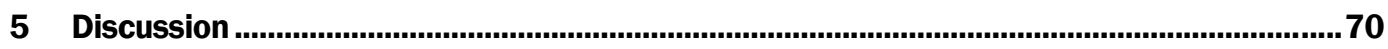

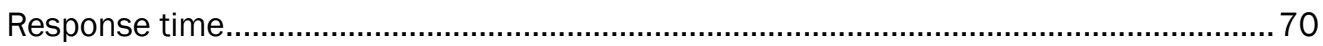

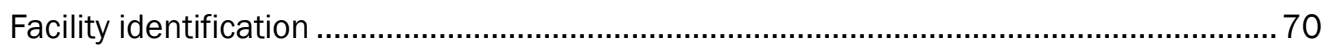

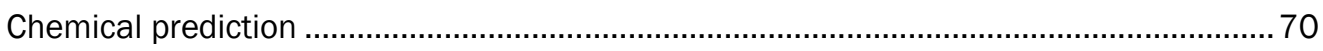

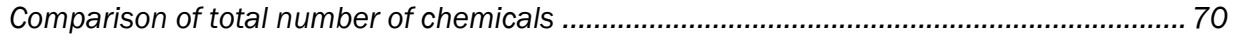


TIC chemical predictions versus chemicals actually reported.............................................. 71

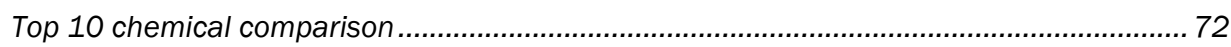

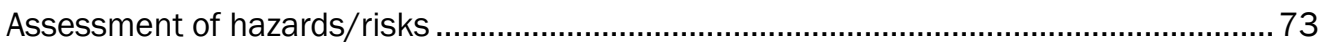

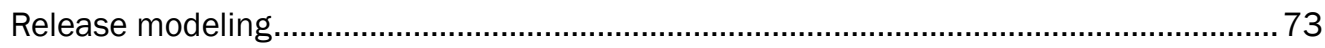

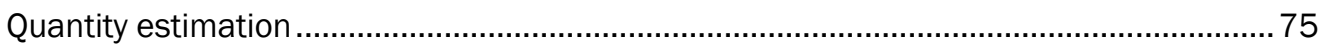

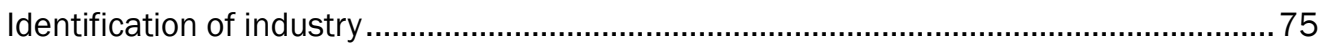

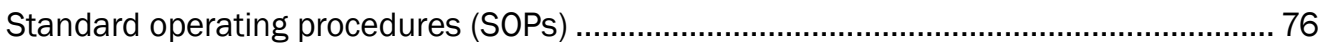

System modification recommendations based on test results ....................................... 76

Feedback from possible user organizations (MANSCEN, the Army Intelligence

Center, Army Geospatial Center, Program Manager for Army Geospatial Data Model).

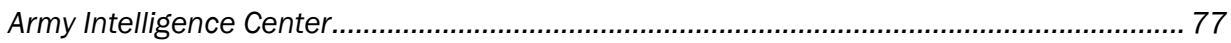

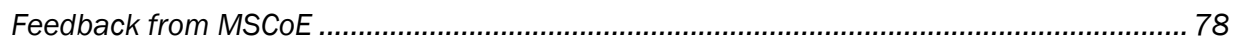

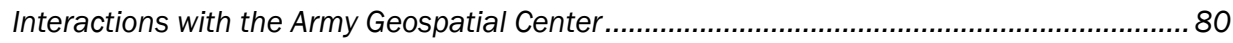

Adaptations for civilian use .................................................................................... 80

6 Conclusions...............................................................................................................................82

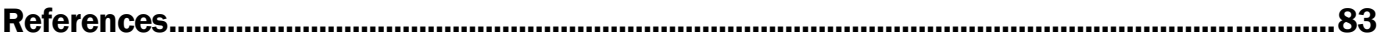

Appendix A: Chemical Database for the TIC-Master Program ................................................86

Appendix B: Instructions on Using EPA TRI Database to Create ICCW ...................................87

Appendix C: Standard Operating Procedures (SOPs) Used in This Study.................................90

Appendix D: TIC-Master Fact Sheet......................................................................................... 242

Appendix E: Email from the Army Intelligence Center .............................................................. 244

Appendix F: White Paper for Modification of TIC-Master for Use by Installations................... 246

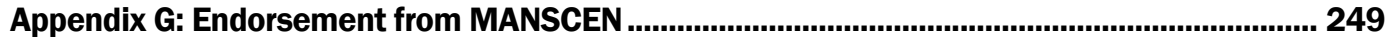

Report Documentation Page 


\section{Figures and Tables}

\section{Figures}

Figure 1. Schematic depicting the use of the TIC-Master program.................................................. 9

Figure 2. Relational TIC data model. ............................................................................................ 18

Figure 3. Pull-down screen showing facility type........................................................................... 19

Figure 4. Phosgene example.............................................................................................. 20

Figure 5. ESRI GIS interface............................................................................................... 21

Figure 6. Location of toxic industrial chemical facilites in the 2009 Warfigher III test area............. 22

Figure 7. Demonstration of the polygon tool to obtain census data. ................................................ 22

Figure 8. Example of part of an SOP for acrolein. …………....................................................... 24

Figure 9. Test scenario submission form. …………….............................................................. 26

Figure 10. Scenario description for Test 1........................................................................... 28

Figure 11. Location map for facility identified in Test 1....................................................................2

Figure 12. Release modeling of an explosion of a 200-gal tank of xylene. The innermost red circle indicates lethality within 60 seconds, and was about $200 \mathrm{~m}$ in diameter. The circular

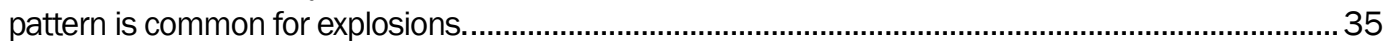

Figure 13. Scenario description for Test 2 …….................................................................. 37

Figure 14. Location map for Test 2. The pink dot is the location of the facility - in the Port

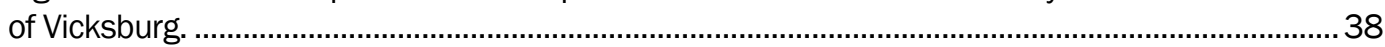

Figure 15. Release modeling of an explosion of a propylene tank for Test 2 ….............................. 42

Figure 16. Scenario description for Test 3.............................................................................. 44

Figure 17. Location map for Test 3. Skull and crossbones symbol marks the identified

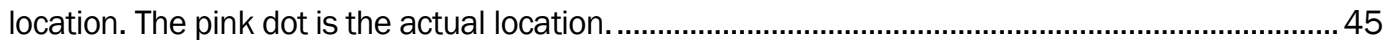

Figure 18. Release modeling supporting Test 3. ......................................................................52

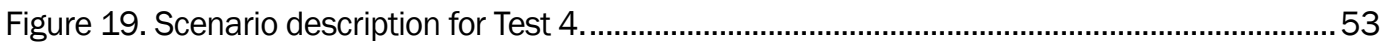

Figure 20. Location map for Test 4. The pink dot is Site A. Skull and crossbones was Site B. ...........54

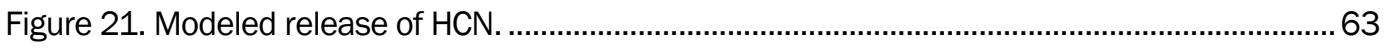

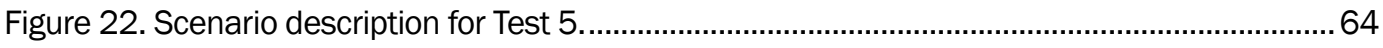

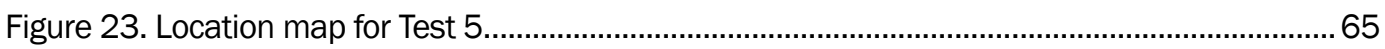

Figure 24. Maps of releases from two facilities from Test 5 scenario. ...............................................68

\section{Tables}

Table 1. Parameters included in chemical spreadsheet................................................................ 10

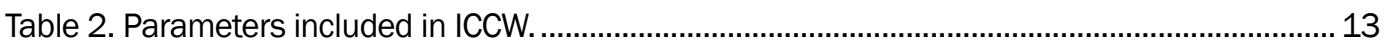

Table 3. Industry types with populated data in ICCW with number of predicted chemicals............. 13

Table 4. List of facilities used for TIC-Master testing...................................................................... 27

Table 5. Chemical identification list for Test 1 scenario. CAS is the chemical abstracts services number for the chemical. 


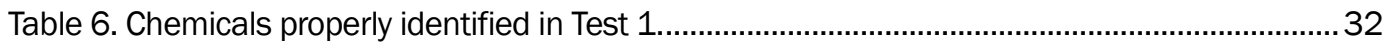

Table 7. Chemicals not precisely identified by the TIC-Master process for Test 1.............................33

Table 8. The 10 most probable chemicals predicted by the TIC-Master database for Test 1.

Chemicals found in the actual inventory are starred..........................................................................3.

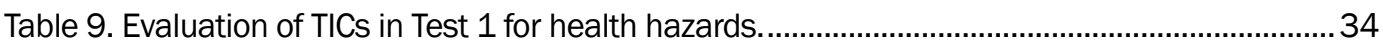

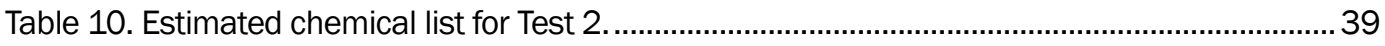

Table 11. Correctly identified chemicals for Test 2 simulation. ........................................................40

Table 12. Comparison of database predicted chemicals with actual chemicals............................. 40

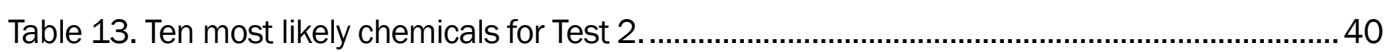

Table 14. Ranking of Test 2 chemicals on fire/flammability: chemicals with NFPA-F ratings

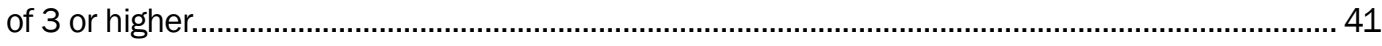

Table 15. Ranking of Test 2 chemicals on toxicity/health: Chemicals with NFPA-H ratings

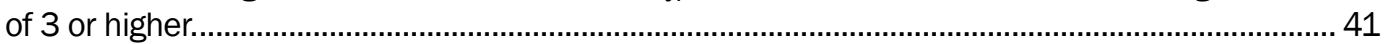

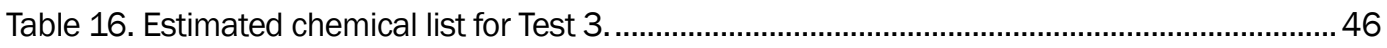

Table 17. Chemicals listed on Nissan Tier II report and the TIC-Master database. .......................... 47

Table 18. Fifteen chemicals identified by the test team appear in the Tier II report and TIC-

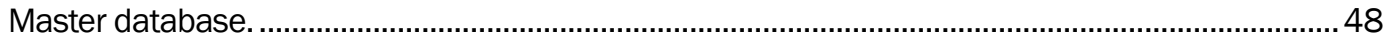

Table 19. Most probable chemicals for Test 3. Starred Items were included in the actual

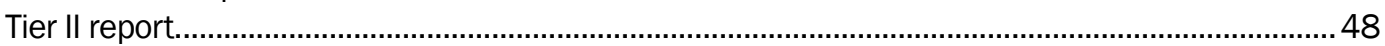

Table 20. Chemical hazard list for test 3 based on fire/flammability (NFPA-F). All

chemicals with rankings of 3 or greater.

Table 21. Chemical hazard list for Test 3 based on toxicity/health (NFPA-H). All chemicals with rankings of 3 or greater. 50

Table 22. Three most significant chemical hazards selected by the test team. .............................. 51

Table 23. Full listing of chemicals predicted for chemical refining, with the first 30 ranked. ..........55

Table 24. Correctly identified chemicals for both Ergon and Clorox................................................... 57

Table 25. TIC chemicals in the Tier II report for Ergon not Identified by TIC-Master. ..........................57

Table 26. Full listing of chemicals predicted for sanitation product manufacturing, with the first 30 ranked. 58

Table 27. TIC chemicals in the Tier II report for Clorox not identified by TIC-Master......................... 59

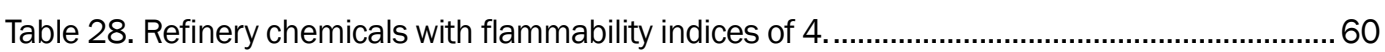

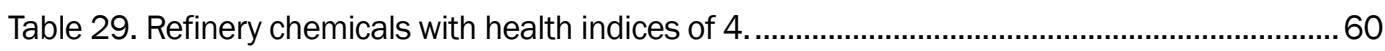

Table 30. Cleaning product chemicals with flammability indices of 4 or higher............................... 61

Table 31. Cleaning product chemicals with health indices of 4 or higher (i indicates insufficient data).

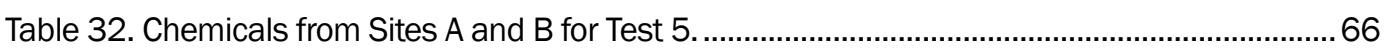

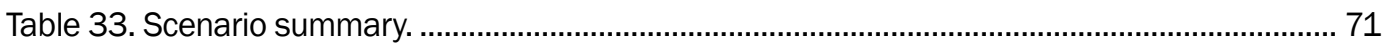




\section{Preface}

This report evaluates the TIC-Master Computer Program. This work was part of a project funded by the Army Environmental Quality/Installations Program titled "Environmental Assessment during the Intelligence Preparation of the Battlefield.” This report was prepared by Dr. Victor F. Medina, Environmental Engineering Branch (EEB), Dr. Dennis L. Brandon, Environmental Risk Assessment Branch (ERAB), Environmental Processes and Engineering Division (EPED), Environmental Laboratory (EL), U.S. Army Engineer Research and Development Center (ERDC), Vicksburg, MS; Dr. K. James Hay, ERDC Construction Engineering Research Laboratory (CERL), Champaign, IL; and Dhiren S. Khona, Army Geospatial Center, Alexandria, VA. Technical reviews were provided by Debbie Felt and Dr. Molly Reif, both of ERDC-EL.

This work was conducted under the general supervision of Andy Martin, Chief, EEB, and Warren Lorentz, Chief, EPED. Dr. Elizabeth Ferguson was the Technical Director.

At the time of publication of this report, Dr. Beth Fleming was Director of EL. COL Kevin J. Wilson was Commander of ERDC, and Dr. Jeffery P. Holland was the Director of ERDC. 


\section{Acronyms}

\%Prob - Percent probability. Used in describing the probability of a chemical being present for a specific industrial process based on analysis conducted by the TIC-Master Database.

AERTA - Army Environmental Requirements and Technology Assessments

AIC - United States Army Intelligence Center (Fort Huachuca)

ALOHA - Areal Locations of Hazardous Atmospheres, a computer program that estimates atmospheric release of chemicals

CAMEO - Computer Aided Management of Emergency Operations

CANUKUS - Canada, the United Kingdom, and the United States. The acronym refers to an international task force consisting of these countries.

CAS - Chemical Abstract Service

CCIR - Commander's Critical Information Requirements

CBRN - Chemical Biological Radiological and Nuclear

CCIR - Commander's Critical Information Requirements

C/JMTK - Commercial Joint Mapping Toolkit

COA: - Course of Action

CoBRA $\AA$ - Chemical Biological Response Aid chemical database

CONUS: - Within the Continental United States

COP - Common Operational Picture 
CDC - Centers for Disease Control

DOA - Department of the Army

DoD - Department of Defense

DPW - Department of Public Works

EPCRA - Emergency Planning and Community Right to Know Act

ERDC - U.S. Army Engineer Research and Development Center

ESRI - Environmental Systems Research Institute. The key vendor of GIS products to US Federal Agencies.

FHP - Force Health Protection

FSO - Full Spectrum Operations

GAO - General Accounting Office

$\mathrm{G}-\mathrm{Gas}$

GIS - Geographic Information Systems

GWOT - Global War on Terror

HPAC - Hazard Prediction and Assessment Capability Model

HPC - Hazard Probability Code. A numeric hazard probability score based on physical state, product, and weapon/accident history

HSC - Hazard Severity Category. A numerical ranking combining maximum hazard from toxicity, flammability, and instability)

HSDB - Hazardous Substances Data Bank

HIFLD-WG - Homeland Infrastructure Foundation Level Database Working Group 
ICCW - Industry/Chemical Crosswalk

ICSC - International Chemical Safety Cards

IPB - Intelligence Preparation of the Battlefield

ITF-25, 40 - International Task Forces 25 and 40, respectively

JEM - Joint Effects Model

L - Liquid

Lat - Latitude

Long - Longitude

LTC - Lieutenant Colonel

MDMP - Military Decision Making Process

MEMA - Mississippi Emergency Management Agency

MSCoE - United States Army Manuever Support Center of Excellence (Fort Leonard Wood)

OCONUS - Outside the Continental United States

NAICS - North American Industrial Classification System

NFPA - National Fire Protection Association. F - Flammability (ranking), $\mathrm{H}$ - Health (ranking)

NIOSH - National Institute for Occupational Safety and Health

NOAA - National Oceanic and Atmospheric Administration

ODUSD(I\&E) - Office of the Deputy Under Secretary of Defense (Installations and the Environment)

RA - Risk Assessment 
RM - Risk Management
RR - Risk Ranking. A ranking combining HSC and HPC parameters.
S - Solid

SOP(s) - Standard Operating Procedure(s)

TIC - Toxic Industrial Chemical

TIM - Toxic Industrial Material

TRI - Toxic Release Inventory

UK - United Kingdom

US - United States

USACE - United States Army Corps of Engineers

USACHPPM or CHPPM - U.S. Army Center for Health Promotion and Preventive Medicine. Now known as the Army Public Health Command.

USBCANZPO - United States, Britain, Canada, Australia, and New Zealand Program Office

USEPA or EPA - U.S. Environmental Protection Agency

USDOA - United States Department of the Army

VFD - City of Vicksburg Fire Department (Vicksburg, MS)

WISER - Wireless Information System for Emergency Responders

WMD - Weapons of Mass Destruction 


\section{Definitions}

Environmental considerations: The spectrum of environmental media, resources, or programs that may impact, or are affected by, the planning and execution of military operations; factors may include, but are not limited to, environmental compliance, pollution prevention, conservation, protection of historical and cultural sites, and protection of flora and fauna (USDoD 2001).

Environmental hazard: All activities that may pollute, create negativenoise-related effects, degrade archeological/cultural resources, or negatively affect threatened or endangered species habitats: also included are environmental health-related hazards.

Environmental stewardship: The integration and application of environmental values into the military mission in order to sustain readiness, improve quality of life, strengthen civil relations, and preserve valuable natural resources (U.S. Department of Defense (USDoD) 2001).

Full Spectrum Operations (FSO): Full spectrum operations describe the combination of offensive, defensive, and stability or civil support operations simultaneously as part of an interdependent joint force to seize, retain, and exploit the initiative, accepting prudent risk to create opportunities to achieve decisive results. They employ synchronized action-lethal and nonlethalproportional to the mission and informed by a thorough understanding of all variables of the operational environment. Mission command that conveys intent and an appreciation of all aspects of the situation guides the adaptive use of Army forces.

Hazard: A condition with the potential to cause injury, or death of personnel; damage to or loss of equipment or property; or mission degradation (USDoD 2001).

Intelligence Preparation of the Battlefield (IPB): IPB is an analytical methodology that is conducted at all levels of command to produce graphical intelligence assessments, estimates, and other intelligence products in support of the commander's decision-making process (United 
States, British, Canadian, Australian and New Zealand Armies' Program Office 2009).

Natural environment: The human ecosystem, including both the physical and biological systems, that provides the resources (i.e. clean air, clean water, healthy surroundings, and sufficient food) necessary to sustain productive human life. Included in the natural resource environment are manmade structures such as water and waste water treatment facilities and natural/cultural resources (U.S. Department of the Army (USDOA) 2004). 


\section{Unit Conversion Factors}

\begin{tabular}{|l|l|l|}
\hline Multiply & By & To Obtain \\
\hline feet & 0.3048 & meters \\
\hline gallons (U.S. liquid) & $3.785412 \mathrm{E}-03$ & cubic meters \\
\hline pounds (force) & 4.448222 & newtons \\
\hline pounds (force) per square inch & 6.894757 & kilopascals \\
\hline pounds (mass) & 0.45359237 & kilograms \\
\hline
\end{tabular}




\section{Introduction}

\section{Intelligence Preparation of the Battlefield}

The Intelligence Preparation of the Battlefield (IPB) is a planning process undertaken in preparation for combat operations. It involves gaining key intelligence that will assist commanders in determining the best way to maneuver their forces and apply firepower to gain maximum advantage over their adversaries. The steps involved with the IPB process are:

1. Define the battlefield environment.

2. Describe the battlefield's effects.

3. Evaluate the threat.

4. Determine threat course of action (COA) (U.S. Department of the Army (USDOA) 1994).

Defining the battlefield environment usually focuses on two key pieces of information. The first is the size, disposition, and condition of the enemy forces. The second focuses on the terrain. Although chemical weapons are a critical part of the IPB process (Center for Counterproliferation Research 2000), industrial chemicals have not traditionally been considered as a substantial threat. However, because of changes in the battle space environment, areas of concentrated industrial chemicals, such as those found at industrial facilities, could cause casualties and impact movement if released or if used intentionally (Hauschild and Lee 2004). An exteme example of the latter was the use of released oil and oil fires by defeated Iraqi forces during the first Gulf War. There has been a report of a chlorine tanker being used in an improvised explosive device attack in Afghanistan; however, in that case, the explosion consumed the chlorine gas, limiting its effect. ${ }^{1}$

\section{Implication of environmental considerations}

The U.S. Army has developed policy that values the interdependence among military missions, the global community, and the environment, which is known as full spectrum operations (FSO). Environmental stewardship represents the reflection of leader and individual awareness of and

\footnotetext{
1 Personal Communication. 2011. LTC Robert Tucker, Fort Leonard Wood, MO.
} 
commitment to protecting the environment. It is a proactive, values-based concept that helps to ensure the sustainability and conservation of resources. Environmental considerations include a myriad of specifics dealing with protection of the natural and cultural environment, as well as environmental considerations in Force Health Protection (FHP). Commanders must integrate environmental considerations into the mission analysis phase of the Military Decision Making Process (MDMP) (Jederberg et al. 2002; USDOA 2010). During the mission analysis, a risk assessment (RA), which includes identifying all environmental-related hazards, is ideally conducted (Jederberg et al. 2002). The RA would identify hazards, which the military planners must anticipate, plan for, and mitigate. The risk management (RM) process is one of controlling risk arising from operational factors and balancing that risk with mission accomplishment (Langenberg 2007). Environmental risks are those risks both to and from the environment that must be included in the RM process.

Many of the factors that affect the health of service members may be directly related to environmental considerations within the theater and to the effects on the environment created by military actions. In many cases, environmental considerations need to be integrated into the IPB process. In certain circumstances, environmental considerations may be significant enough to be included in the commander's critical information requirements (CCIR). These environmental considerations will impact all levels of command and should be integrated, as appropriate, into the planning process (USDOA 2010).

\section{Urban battlefield}

Discussions conducted with experts from both the Army Maneuver Support Center of Excellence (MSCoE) and the Army Intelligence Center (AIC) indicated that before the Global War on Terror (GWOT), including Operations Enduring Freedom and Iraqi Freedom, American military doctrine was focused on the destruction of foreign standing armies using rapid movement in open spaces. This process was brilliantly displayed during the First Gulf War (Operation Desert Storm), when coalition forces used maneuver and concentrated firepower to route Iraqi forces that had invaded Kuwait. Urban spaces were considered unsuitable for this type of combat model. The use of maneuver and firepower to defeat hostile forces was again successfully demonstrated in the early phases of the liberation of Afghanistan (Operation Enduring Freedom) and the invasion of Iraq (Operation Iraqi Freedom or the Second Gulf War). However, as operations 
in both Iraq and Afghanistan continued, American and coalition forces have had to engage in operations in urban environments to deal with insurgents. The U.S. Army has had to develop new strategies, weapons, and equipment for this challenging environment. Urban environments frequently have industrial centers where industrial chemicals are used. Urban operations increase the possibility of troop exposure to industrial chemicals, as well as their release during operations.

\section{Toxic industrial chemicals}

Chemicals are a part of modern industry. Production of virtually all goods requires at least some chemical processes. The tenets of toxicology indicate that any chemical can be toxic, as it is just a matter of dosage. However, some chemicals are clearly more problematic than others. One category of chemicals that is of particular concern is the toxic industrial chemicals (TICs) category.

TICs are a special group of chemicals that are deemed of great threat to human health and have the possibility of being weaponized. TICs have been defined by two international task forces (ITFs). The first was ITF-25, which was started in 1994 and consisted of three countries, the United States, the United Kingdom (UK), and Canada (CANUKUS 1994). ITF-25 created a prioritized list of industrial chemicals for military use to assist in identifying, assessing, and controlling exposure risks from industrial chemicals in the battlefield (Steumpfle et al. 1996). This database identifies whether chemicals are listed on the ITF-25 priority list and to what degree (low, medium, or high). The ITF-25 assessed chemicals primarily based on two criteria, toxicity and producibility, which favor chemicals that are acute inhalation hazards produced in large quantities. The ITF-25 priority list consists of 98 chemicals that met certain hazard index criteria. Resta (2001) discussed the activities of international task forces (ITFs, consisting of the United States, the United Kingdom, and Canada) to define and evaluate toxic industrial chemicals. He emphasized the need to evaluate more chemicals than the number included in the first (ITF-25) agreement, which ultimately resulted in ITF-40.

The ITF-40 (which was expanded to include Australia and New Zealand) reviewed, updated, and enhanced the methodology of the ITF-25 work to produce a new list of prioritized industrial chemicals (U.S. Army Center for Health Promotion and Preventive Medicine (USACHPPM) 2003; U.S. British, Canadian, Australian, and New Zealand Armies' Program Office 
(USBCANZPO) 2009). The task force determined that the primary deficiency of the ITF-25 list is that it did not consider other important hazardous chemical properties, such as reactivity, ignitability, or corrosiveness. In particular, the task force recognized that a large number of industrial disasters have involved flammable chemicals. ITF-40 attempted to correct this deficiency as well as making several other improvements in ranking hazards and increasing the number of evaluated chemicals. The ITF-40 database includes 1756 chemicals, 1042 of which are ranked, and 63 of which are ranked high enough to be listed as military priority industrial chemical hazards.

Several sources have emphasized the threat of TICs during military operations. In a Master's thesis for the Army Command and General Staff College, Small (2002) concluded "that in the hands of terrorists, aggressive states or nonstate actors, TICs will serve as WMD (weapons of mass destruction), undermine regional stability, and threaten US interests." Small (2002) concluded that joint doctrine for combat and noncombat operations inadequately takes account of current TIC realities. Based on the documents cited in this thesis, "the US Armed Forces have not collectively identified overall force structure requirements for dealing with this threat." In 1998, the Government Accounting Office (GAO) concluded that DoD had not developed doctrine that addressed low- level exposures to chemical agents found on the battlefield. DoD has initiated research to identify the cause (environmental exposures) and effects (medical outcome) relationships associated with various chemicals (Hauschild 2004).

The USBCANZPO (2009) stated that the presence and threat of TICs continues to increase. In environments where normal security regulation has broken down, the failure to secure TIM, CBRN weapons, or precursors, may have significant security or health implications for military personnel. Identification and security of such materials will need to be addressed. This will be especially relevant to operations conducted in complex urban and suburban terrain. As a recent example, senators have asked the Pentagon's inspector general to investigate the precise circumstances surrounding the apparent exposure of Army National Guard soldiers to the chemical sodium dichromate at an industrial facility in Iraq. The issues debated include 1) the Army's role in warning soldiers about the contamination, 2) the personal protective gear provided, and 3) the subsequent testing of soldiers to determine the health impact of the exposure (USEPA 2009). 
The USBCANZPO (2009) also provides a preliminary planning guide to enhance preparation, resourcing, and the conduct of operations to mitigate the effects of CBRN/TIM hazards in order to conduct effective operations across the spectrum of conflict. The following CBRN/TIM factors need to be incorporated into the planning process:

a. Meteorology.

b. National Infrastructure.

1) Population and industrial centers.

2) Communications (air, road, rail, waterway transportation).

3) Energy resource demands (oil, electricity, gas, nuclear and water).

4) Emergency response capability (mitigation and containment).

Hill (2009) set forth the Army Environmental Requirements and Technology Assessments (AERTA), which represent the critical environmental technology needs for accomplishing the Army's mission while minimizing impact to the environment. These requirements are Army-level requirements and include installation needs only when the need is critical to the execution of the Army's mission. TICs in FSO are applicable to two AERTAs: ER-6-07-01, "Environmental Restoration during Full Spectrum Operations (FSO)," and ER-10-07-01 “Avoidance of Environmental Risk during Contingency Operations” (Hill 2009).

The combined military services have identified TICs as an increasing hazard to military operations. Cutclasure and McClellan (2009) prepared a briefing discussing a Joint Program to improve assessment of TIC hazards in order to improve consequence management approaches.

In addition, the Chief of the U.S. Army Corps of Engineers (USACE), General R.L Van Antwerp, directed the COE to revise policies to evaluate hazardous and toxic agents that may be encountered with overseas contingency operations (Van Antwerp 2010). This further indicates the recognition that these chemicals could present significant hazards to U.S. forces and surrounding native populations.

\section{Emergency Planning and Community Right to Know Act}

The Emergency Planning and Community Right to Know Act (EPCRA) was passed in 1986 and has been amended and reauthorized several times since then. 


\section{Community Right to Know Reporting (EPA Tier II Reporting)}

The EPCRA requires industrial facilities to annually report their hazardous and toxic chemicals to the USEPA, the State agency equivalent, and to local emergency responders, particularly fire departments (Cornell University Law School (CULS), undated; USEPA 2010). Industries that maintain either $10,000 \mathrm{lb}$ of total hazardous chemicals or $500 \mathrm{lb}$ of extremely hazardous materials must conduct inventory reporting.

The reports consist of two parts. Tier I reports must be submitted by 1 March of each year and include total chemicals for specific hazardous chemical categories. Tier II reporting is much more detailed, involving specific hazardous chemicals and their quantities. The definition of hazardous chemicals is quite broad involving lists from other regulations as well as a criteria definition. Thresholds exist for the chemicals, which vary from $1000 \mathrm{~kg}$ to as little as $1 \mathrm{~kg}$ for the most hazardous. If the chemical is below the threshold, the company is not required to report it.

Tier II reports are an excellent, in fact definitive, source of chemical information for a specific facility, as a wide range of chemicals are reported. One limitation is that if the chemical is not above the threshold, it does not have to be reported. The good news is that most facilities will take a conservative approach and report a below-threshold chemical if (1) there is any chance they might reach or get near the threshold in order to avoid a penalty, and (2) as a courtesy to local emergency responders. A second limitation is that companies can withhold some specific chemicals if they are trade secrets. They do, however, have to report the quanitity and properties of any trade secret chemicals.

As mentioned, Tier II reports are valuable records in that they define the chemicals used by a specific facility. Unfortunately, as of this date, Tier II reports are not digital. This means there is no means of easily searching them. This limits their effectiveness in terms of determining chemicals generated from an industry.

\section{Toxic Release Inventory (TRI) reporting}

Another component of EPCRA requires industries to file annual reports of chemical releases and wastes. There are approximately 650 chemicals and chemical categories that are subject to reporting by these industries. The USEPA maintains this collected data in the Toxic Release Inventory (TRI), 
where the public may view and analyze the data in many different ways. For example, the data may be sorted by facility, geographical location, chemical, and industry. The ability to search these data makes this a valuable resource for predicting chemicals used at industrial facilities. That said, the TRI database does have some key limitations. The chemicals list is relatively small (about 650 chemicals). Second, the chemicals identified are limited only to those released over the timeframe searched.

\section{The TIC-Master Project}

TIC exposures may result during military urban operations involving industrial or manufacturing facilities. Even if soldiers are adequately protected, TIC releases can affect neutral or friendly populations in the environs of an urban operation. Better understanding and mapping of the types of TICs that may be present in a battlefield from industrial sources are needed as an important part of an assessment tool that could be incorporated into the IPB Process. A database/GIS system known as the TIC-Master program was built containing over 2,000 TICs, primarily from the ITF-40 and EPA TRI. The database included physical properties, hazard assessment information, and environmental effects and remediation information. A data model was also constructed to relate these chemicals and industrial types using the 6-digit codes from the North American Industrial Classification System (NAICS). Data to relate the chemicals to the industries was queried from the TRI database. Analysis of the data allows for a crude estimation of the probability to encounter specific TICs at a particular industrial facility. A custom GIS standards-based platform has been designed to specifically map industrial facilities within a designated region. The application allows relevant information about the chemical(s) to be queried from the TIC database for a specific site and the mapping of areas of highest hazard probability. The industrial chemical database has been integrated with a GIS mapping method. Testing compared the prediction of primary industrial chemicals at specific industrial locations within the geographical region to the actual chemicals and locations. 


\section{TIC-Master Program}

\section{Approach}

In order to evaluate the capability to estimate TICs associated with the industrial process during the IPB process, a computer-based program was created, which will be referred to as the TIC-Master program. This program consists of a database of chemical information based on lists of TICs identified by the ITF-40 and the EPA TRI. This database was then crosslinked to a database that related the chemicals to industries and industrial processes. Finally, this database was placed in a geographic information system (GIS) that allowed for mapping of the facility (or facilities) in an environment that allowed users to identify features in spatial proximity to the site(s), and model releases.

Figure 1 shows how the TIC-Master would be used to improve the IPB process. A course of action (COA) would be proposed. In this case, COA 1 involves interaction with an industrial facility suspected of using TICs. The TIC-Master program will be used to identify TICs associated with the industry, and will also be used to determine some release scenarios. In this case, the pink circles indicate effects of potential chemical releases. It turns out that COA 1 does involve possible exposure to the chemical hazard (be it toxicity, explosion, or fire). Two other possibilities are also evaluating COA 2 and COA 3. COA 2 does involve a possible chemical hazard, but not as significant as COA 1. COA 3 avoids all. However, other considerations may make COA 1 the COA chosen. If so, the TIC-Master can provide chemical information to assist in preparing personnel. Also, environmental standard operating procedures can be used to address environmental issues that may arise from the operation.

\section{TIC Database}

The purpose of the TIC database was to provide hazard information for TICs that may be found in the battlefield and the necessary industrial information for the TIC-Master to provide a reasonable prediction of potential TICs that may be found at a particular type of industrial site. The TIC database was electronically created as a Microsoft Office 97-2003 Excel worksheet file consisting of several spreadsheets, each with a different purpose (Appendix A). For integration into the TIC Master, two spreadsheets were 


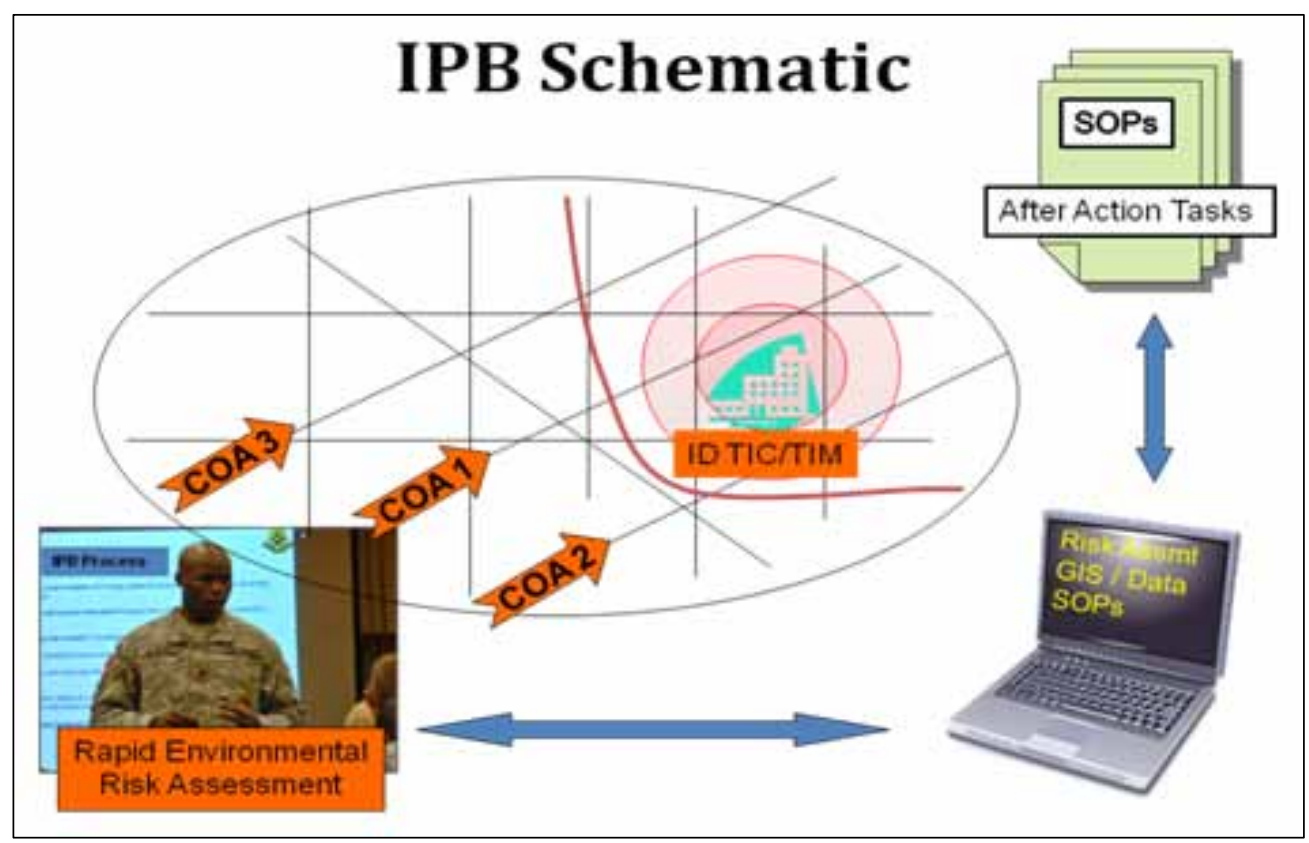

Figure 1. Schematic depicting the use of the TIC-Master program.

utilized. One contains the physical properties and hazard assessment information for each chemical and the other includes a list of industrial types and potential chemicals associated with those industries (industry/chemical crosswalk or ICCW).

\section{Chemical information}

The chemical spreadsheet was created based on a list of 1756 chemicals identified by the ITF-40, (USACHPPM 2003) and is supplemented by 578 listed chemicals from the USEPA Toxic Release Inventory (TRI) giving a total size of 2092 chemicals (242 chemicals are common between the two lists). For comparison, GeoRAMS, a system designed to model chemical spills that was developed for the U.S. Army, had a library of 44 chemicals (U.S. Army Engineer Research and Development Center (ERDC) - Environmental Laboratory (EL) 2005). Chemicals are presented in ascending order based on their Chemical Abstract Service (CAS) registry number. The spreadsheet includes 26 columns/parameters for each chemical. These include four identifying columns: Chemical ID, CAS, CAS Text, and Chemical Name. Eight parameters are designated for physical properties that include physical state, color, odor, storage state, melting point, boiling point, vapor density, and water solubility. The remaining 14 columns provide hazard assessment data. The hazard assessment columns include National Fire Protection Association (NFPA 2006) diamond ratings; data provided directly from the ITF-40 database; 
indicators as to whether the chemical is regarded as a high priority chemical by ITF-40 and ITF-25 (Steumpfle et al. 1996), which was the predecessor task group to ITF-40; and a column for any special considerations to be noted. Each parameter is described in Table 1.

Table 1. Parameters included in chemical spreadsheet.

\begin{tabular}{|c|c|c|}
\hline Parameter & Data Type & Description \\
\hline Chemical_ID & Integer & Primary key for chemical in ascending order \\
\hline CAS & Integer & $\begin{array}{l}\text { Chemical Abstract Service registry number in integer form } \\
\text { (minus hyphens) }\end{array}$ \\
\hline CAS_Text & Text & $\begin{array}{l}\text { Chemical Abstract Service registry number in text form as } \\
\text { commonly presented with hyphens }\end{array}$ \\
\hline Chemical_Name & Text & $\begin{array}{l}\text { Chemical name, usually a commonly accepted systematic } \\
\text { name }\end{array}$ \\
\hline State & Text & $\begin{array}{l}\text { Physical state near } 25 \text { degrees Centigrade, listed as G (gas), } \\
\text { L (liquid), S (solid), or any combination of the three }\end{array}$ \\
\hline Color & Text & $\begin{array}{l}\text { Color in the visible spectrum, listed as "no" or simple color } \\
\text { description }\end{array}$ \\
\hline Odor & Text & Odor description, listed as “no”, “yes”, or simple description \\
\hline Storage_State & Text & $\begin{array}{l}\text { Likely state that chemical will be stored: S (solid), L (liquid in } \\
\text { closed container or cylinder), G (compressed gas in vessel), } \\
\text { SL (solid or liquid in closed container, LG (liquefied } \\
\text { compressed gas in vessel), GLG (liquefied or non-liquefied } \\
\text { gas in vessel), GIG (compressed gas diluted with inert gases), } \\
\text { GAC (compressed gas dissolved in acetone), A (aqueous } \\
\text { liquid solution), Ssol (Solid or liquid solution other than } \\
\text { aqueous), SA (solid or aqueous liquid solution) }\end{array}$ \\
\hline Melting_Point & Numeric & Melting point in degrees Centigrade \\
\hline Boiling_Point & Numeric & Boiling point in degrees Centigrade \\
\hline Vapor_Density & Numeric & Relative vapor density (air $=1.0$ ) \\
\hline Water_Solubility & Text & $\begin{array}{l}\text { Simple description of water solubility (e.g., insoluble, poor, } \\
\text { very good, miscible, reaction) }\end{array}$ \\
\hline Exposure_Route & Text & Primary exposure route risk as determined by ITF- 40 \\
\hline NBCFilter_Protection & Text & $\begin{array}{l}\text { NBC filter protection as provided by FM 3-11.4, Table E-1: E } \\
\text { (effective), M (marginal), and P (poor) }\end{array}$ \\
\hline NFPA_H & Integer & $\begin{array}{l}\text { NFPA } 704 \text { health hazard rating }(0 \text { to } 4) \text {; or in cases where } \\
\text { NFPA } 704 \text { health rating were not inputted, ITF- } 40 \\
\text { health/toxicity score based on highest toxicity rating for three } \\
\text { potential exposure routes ( } 1 \text { to } 4)\end{array}$ \\
\hline NFPA_F & Integer & NFPA 704 flammability hazard rating (0 to 4 ) \\
\hline
\end{tabular}




\begin{tabular}{|l|l|l|}
\hline Parameter & Data Type & Description \\
\hline NFPA_I & Integer & NFPA 704 instability hazard rating (0 to 4) \\
\hline NFPA_Special & Text & $\begin{array}{l}\text { NFPA 704 special hazard, limited to W (reacts violently or } \\
\text { explosively with water) and OX (possesses oxiziding } \\
\text { properties) }\end{array}$ \\
\hline HPV & $\begin{array}{l}\text { Text } \\
\text { (Boolean) }\end{array}$ & $\begin{array}{l}\text { High production volume as imported from ITF-40 data: yes or } \\
\text { no }\end{array}$ \\
\hline ITF25_Hazard & Text & ITF -25 hazard rating: low, medium, or high \\
\hline ITF40_Priority & $\begin{array}{l}\text { Text } \\
\text { (Boolean) }\end{array}$ & $\begin{array}{l}\text { Indication of inclusion in ITF-40 military priority industrial } \\
\text { chemical hazards, table II-13 (ref): yes or no }\end{array}$ \\
\hline ITF40_HSC & Text & $\begin{array}{l}\text { ITF-40 Hazard Severity Category: maximum hazard rank from } \\
\text { ITF-40 health/toxicity, NFPA704 flammability, and NFPA 704 } \\
\text { instability (negligible, marginal, critical, catastrophic) }\end{array}$ \\
\hline ITF40_HPC & Text & $\begin{array}{l}\text { ITF-40 Hazard Probability Category: summed probability rank } \\
\text { based on physical state, production, and history (unlikely, } \\
\text { seldom, occasional, likely, or frequent) }\end{array}$ \\
\hline ITF40_RR & Text & $\begin{array}{l}\text { ITF-40 Risk Ranking: matrix application of ITF-40 HSC and } \\
\text { ITF-40 HPC to provide overall risk (low, moderate, high, or } \\
\text { extreme) }\end{array}$ \\
\hline ITF40_History & Text & ITF-40 history converted to text (accident, listed, or weapon) \\
\hline Considerations & Text & \begin{tabular}{l} 
Comments that may provide pertinent additional information \\
\hline
\end{tabular} \\
\hline
\end{tabular}

Physical property data and NFPA hazard ratings were collected through the ChemIDplus Lite data searching tool provided by the United States National Library of Medicine (http://chem.sis.nlm.nih.gov/chemidplus/chemidlite.jsp). This tool allows a search for chemical information from numerous databases. Of these databases, there were four that provided pertinent physical property data included in this spreadsheet: National Institute for Occupational Safety and Health (NIOSH) International Chemical Safety Cards (NIOSH, undated), NIOSH Pocket Guide to Chemical Hazards (NIOSH 2005), Computer Aided Management of Emergency Operations (CAMEO), and the National Library of Medicine Hazardous Substances Data Bank (HSDB). It should be noted that sometimes the data within these sources are conflicting and best judgment was used to decide on the values entered into the chemical spreadsheet. 


\section{Industry/chemical crosswalk}

The industries provided in the industry/chemical crosswalk (ICCW) are listed by the six-digit North American Industrial Classification System (NAICS) codes, which were obtained via http://www.census.gov/eos/www/naics/. There are 1,175 six-digit codes, of which 126 were populated with data.

The ICCW provides data that can be used to predict chemicals that may be present at a particular industrial site if the six-digit NAICS code is known or can be determined. Chemical/industry relationships were developed by reviewing the USEPA TRI data for given industries. Custom queries were performed for each six-digit code to list all reported releases of chemicals by a given industry type. These data allow for the generation of a list of chemicals reported, the number of facilities reporting, the frequency a particular chemical is reported, and the probability that a particular chemical will be reported as released by a facility that can be identified by that particular six-digit NAICS code. If the chemicals and probabilities generated by this procedure are assumed to be characteristic of this industry type, then these data can be used to predict chemicals and their likelihood of being found at this type facility. It should be noted that custom queries used to populate the data in the ICCW were performed during May and June 2009 utilizing the TRI database that was current at that time. The exact procedure used to gather and manipulate these data is provided in Appendix B.

Several limitations are associated with the method for populating the ICCW. For each six-digit NAICS code, there may be many subtypes of industries. This means that chemical lists generated will contain possible chemicals for all the subtypes under a particular industry type, which in many cases will be excessive. The chemicals list generated will only contain TRI listed chemicals, of which there are 578 (only includes chemicals with an assigned CAS and does not include chemical mixtures). So, many chemicals that are in the chemical spreadsheet will not be included in a prediction of chemicals. Also, the TRI data only include data from industrial sites located within the United States, which may limit applicability to other countries.

The industry/chemical crosswalk spreadsheet contains six data columns: ICCW ID, NAICS Code, NAICS Title, CAS, TRICHEM Name, and TRI\% Prob. These parameters are defined in Table 2. Table 3 lists the industries with populated data in the industry/chemical crosswalk with the number of 
Table 2. Parameters included in ICCW.

\begin{tabular}{|l|l|l|}
\hline Parameter & Value & Description \\
\hline ICCW_ID & Integer & $\begin{array}{l}\text { Primary key for industry/chemical relationship in crosswalk, } \\
\text { in ascending order }\end{array}$ \\
\hline NAICS_Code & Integer & 6-digit NAICS code identifying industry \\
\hline NAICS_Title & Text & Text description of NAICS industry listing \\
\hline CAS & Integer & $\begin{array}{l}\text { Chemical Abstract Service registry number in integer form } \\
\text { (minus hyphens) }\end{array}$ \\
\hline TRICHEM_NAME & Text & Chemical name as provided by USEPA TRI database \\
\hline TRI\%_Prob & Numeric & $\begin{array}{l}\text { Probability that chemical was reported in the USEPA TRI } \\
\text { database by a facility associated with the 6-digit NAICS code }\end{array}$ \\
\hline
\end{tabular}

Table 3. Industry types with populated data in ICCW with number of predicted chemicals.

\begin{tabular}{|c|c|c|}
\hline NAICS Code & NAICS Title & \# Chemicals \\
\hline 212319 & Other Crushed and Broken Stone Mining and Quarrying & 1 \\
\hline 212324 & Kaolin and Ball Clay Mining & 11 \\
\hline 221112 & Fossil Fuel Electric Power Generation & 76 \\
\hline 221310 & Water Supply and Irrigation Systems & 6 \\
\hline 221320 & Sewage Treatment Facilities & 6 \\
\hline 311119 & Other Animal Food Manufacturing & 37 \\
\hline 311222 & Soybean Processing & 26 \\
\hline 311225 & Fats and Oils Refining and Blending & 25 \\
\hline 311421 & Fruit and Vegetable Canning & 28 \\
\hline 311511 & Fluid Milk Manufacturing & 18 \\
\hline 311615 & Poultry Processing & 41 \\
\hline 311919 & Other Snack Food Manufacturing & 25 \\
\hline 311991 & Perishable Prepared Food Manufacturing & 9 \\
\hline 312111 & Soft Drink Manufacturing & 21 \\
\hline 321113 & Sawmills & 36 \\
\hline 321114 & Wood Preservation & 43 \\
\hline 321211 & Hardwood Veneer and Plywood Manufacturing & 26 \\
\hline 321918 & Other Millwork (including Flooring) & 26 \\
\hline 321991 & Manufactured Home (Mobile Home) Manufacturing & 30 \\
\hline 322121 & Paper (except Newsprint) Mills & 90 \\
\hline 322130 & Paperboard Mills & 81 \\
\hline 322211 & Corrugated and Solid Fiber Box Manufacturing & 33 \\
\hline
\end{tabular}




\begin{tabular}{|c|c|c|}
\hline NAICS Code & NAICS Title & \# Chemicals \\
\hline 322212 & Folding Paperboard Box Manufacturing & 34 \\
\hline 322214 & Fiber Can, Tube, Drum, and Similar Products Manufacturing & 24 \\
\hline 324110 & Petroleum Refineries & 158 \\
\hline 324121 & Asphalt Paving Mixture and Block Manufacturing & 42 \\
\hline 324191 & Petroleum Lubricating Oil and Grease Manufacturing & 105 \\
\hline 324199 & All Other Petroleum and Coal Products Manufacturing & 75 \\
\hline 325110 & Petrochemical Manufacturing & 182 \\
\hline 325120 & Industrial Gas Manufacturing & 137 \\
\hline 325131 & Inorganic Dye and Pigment Manufacturing & 71 \\
\hline 325132 & Synthetic Organic Dye and Pigment Manufacturing & 105 \\
\hline 325181 & Alkalies and Chlorine Manufacturing & 173 \\
\hline 325182 & Carbon Black Manufacturing & 39 \\
\hline 325188 & All Other Basic Inorganic Chemical Manufacturing & 285 \\
\hline 325191 & Gum and Wood Chemical Manufacturing & 88 \\
\hline 325192 & Cyclic Crude and Intermediate Manufacturing & 140 \\
\hline 325193 & Ethyl Alcohol Manufacturing & 52 \\
\hline 325199 & All Other Basic Organic Chemical Manufacturing & 286 \\
\hline 325211 & Plastics Material and Resin Manufacturing & 277 \\
\hline 325212 & Synthetic Rubber Manufacturing & 136 \\
\hline 325221 & Cellulosic Organic Fiber Manufacturing & 89 \\
\hline 325222 & Noncellulosic Organic Fiber Manufacturing & 112 \\
\hline 325311 & Nitrogenous Fertilizer Manufacturing & 76 \\
\hline 325312 & Phosphatic Fertilizer Manufacturing & 32 \\
\hline 325314 & Fertilizer (Mixing Only) Manufacturing & 77 \\
\hline 325320 & Pesticide and Other Agricultural Chemical Manufacturing & 330 \\
\hline 325411 & Medicinal and Botanical Manufacturing & 156 \\
\hline 325412 & Pharmaceutical Preparation Manufacturing & 171 \\
\hline 325413 & In-Vitro Diagnostic Substance Manufacturing & 22 \\
\hline 325414 & Biological Product (except Diagnostic) Manufacturing & 32 \\
\hline 325510 & Paint and Coating Manufacturing & 156 \\
\hline 325520 & Adhesive Manufacturing & 166 \\
\hline 325611 & Soap and Other Detergent Manufacturing & 120 \\
\hline 325612 & Polish and Other Sanitation Good Manufacturing & 113 \\
\hline 325613 & Surface Active Agent Manufacturing & 121 \\
\hline
\end{tabular}




\begin{tabular}{|c|c|c|}
\hline NAICS Code & NAICS Title & \# Chemicals \\
\hline 325620 & Toilet Preparation Manufacturing & 60 \\
\hline 325910 & Printing Ink Manufacturing & 64 \\
\hline 325920 & Explosives Manufacturing & 57 \\
\hline 325991 & Custom Compounding of Purchased Resins & 87 \\
\hline 325992 & $\begin{array}{l}\text { Photographic Film, Paper, Plate, and Chemical } \\
\text { Manufacturing }\end{array}$ & 105 \\
\hline 325998 & $\begin{array}{l}\text { All Other Miscellaneous Chemical Product and Preparation } \\
\text { Manufacturing }\end{array}$ & 268 \\
\hline 326140 & Polystyrene Foam Product Manufacturing & 46 \\
\hline 326150 & $\begin{array}{l}\text { Urethane and Other Foam Product (except Polystyrene) } \\
\text { Manufacturing }\end{array}$ & 71 \\
\hline 326160 & Plastics Bottle Manufacturing & 25 \\
\hline 326191 & Plastics Plumbing Fixture Manufacturing & 32 \\
\hline 326192 & Resilient Floor Covering Manufacturing & 38 \\
\hline 326199 & All Other Plastics Product Manufacturing & 116 \\
\hline 326299 & All Other Rubber Product Manufacturing & 95 \\
\hline 327112 & $\begin{array}{l}\text { Vitreous China, Fine Earthenware, and Other Pottery Product } \\
\text { Manufacturing }\end{array}$ & 15 \\
\hline 327215 & Glass Product Manufacturing Made of Purchased Glass & 51 \\
\hline 327332 & Concrete Pipe Manufacturing & 12 \\
\hline 327390 & Other Concrete Product Manufacturing & 35 \\
\hline 327910 & Abrasive Product Manufacturing & 48 \\
\hline 331111 & Iron and Steel Mills & 100 \\
\hline 331221 & Rolled Steel Shape Manufacturing & 51 \\
\hline 331316 & Aluminum Extruded Product Manufacturing & 55 \\
\hline 331319 & Other Aluminum Rolling and Drawing & 42 \\
\hline 331422 & Copper Wire (except Mechanical) Drawing & 54 \\
\hline 331491 & $\begin{array}{l}\text { Nonferrous Metal (except Copper and Aluminum) Rolling, } \\
\text { Drawing, and Extruding }\end{array}$ & 63 \\
\hline 332311 & Prefabricated Metal Building and Component Manufacturing & 50 \\
\hline 332312 & Fabricated Structural Metal Manufacturing & 68 \\
\hline 332321 & Metal Window and Door Manufacturing & 62 \\
\hline 332439 & Other Metal Container Manufacturing & 69 \\
\hline 332812 & $\begin{array}{l}\text { Metal Coating, Engraving (except Jewelry and Silverware), } \\
\text { and Allied Services to Manufacturers }\end{array}$ & 85 \\
\hline 332813 & Electroplating, Plating, Polishing, Anodizing, and Coloring & 102 \\
\hline
\end{tabular}




\begin{tabular}{|c|c|c|}
\hline NAICS Code & NAICS Title & \# Chemicals \\
\hline 332911 & Industrial Valve Manufacturing & 34 \\
\hline 332912 & Fluid Power Valve and Hose Fitting Manufacturing & 28 \\
\hline 332919 & Other Metal Valve and Pipe Fitting Manufacturing & 42 \\
\hline 333319 & $\begin{array}{l}\text { Other Commercial and Service Industry Machinery } \\
\text { Manufacturing }\end{array}$ & 41 \\
\hline 333518 & Other Metalworking Machinery Manufacturing & 21 \\
\hline 333911 & Pump and Pumping Equipment Manufacturing & 46 \\
\hline 333924 & $\begin{array}{l}\text { Industrial Truck, Tractor, Trailer, and Stacker Machinery } \\
\text { Manufacturing }\end{array}$ & 53 \\
\hline 333991 & Power-Driven Handtool Manufacturing & 30 \\
\hline 334111 & Electronic Computer Manufacturing & 42 \\
\hline 334513 & $\begin{array}{l}\text { Instruments and Related Products Manufacturing for } \\
\text { Measuring, Displaying, and Controlling Industrial Process } \\
\text { Variables }\end{array}$ & 36 \\
\hline 335122 & $\begin{array}{l}\text { Commercial, Industrial, and Institutional Electric Lighting } \\
\text { Fixture Manufacturing }\end{array}$ & 33 \\
\hline 335211 & Electric Housewares and Household Fan Manufacturing & 29 \\
\hline 335311 & $\begin{array}{l}\text { Power, Distribution, and Specialty Transformer } \\
\text { Manufacturing }\end{array}$ & 50 \\
\hline 335313 & Switchgear and Switchboard Apparatus Manufacturing & 46 \\
\hline 335911 & Storage Battery Manufacturing & 36 \\
\hline 335929 & Other Communication and Energy Wire Manufacturing & 47 \\
\hline 335931 & Current-Carrying Wiring Device Manufacturing & 51 \\
\hline 335999 & $\begin{array}{l}\text { All Other Miscellaneous Electrical Equipment and } \\
\text { Component Manufacturing }\end{array}$ & 58 \\
\hline 336111 & Automobile Manufacturing & 91 \\
\hline 336370 & Motor Vehicle Metal Stamping & 61 \\
\hline 336399 & All Other Motor Vehicle Parts Manufacturing & 101 \\
\hline 336419 & $\begin{array}{l}\text { Other Guided Missile and Space Vehicle Parts and Auxiliary } \\
\text { Equipment Manufacturing }\end{array}$ & 23 \\
\hline 336612 & Boat Building & 52 \\
\hline 336999 & All Other Transportation Equipment Manufacturing & 40 \\
\hline 337122 & Nonupholstered Wood Household Furniture Manufacturing & 57 \\
\hline 337124 & Metal Household Furniture Manufacturing & 30 \\
\hline 337127 & Institutional Furniture Manufacturing & 61 \\
\hline 337211 & Wood Office Furniture Manufacturing & 50 \\
\hline 337214 & Office Furniture (except Wood) Manufacturing & 49 \\
\hline
\end{tabular}




\begin{tabular}{|l|l|l|}
\hline NAICS Code & NAICS Title & \# Chemicals \\
\hline $\mathbf{3 3 7 9 2 0}$ & Blind and Shade Manufacturing & 32 \\
\hline $\mathbf{3 3 9 9 9 9}$ & All Other Miscellaneous Manufacturing & 108 \\
\hline $\mathbf{4 2 4 4 1 0}$ & General Line Grocery Merchant Wholesalers & 3 \\
\hline $\mathbf{4 2 4 6 9 0}$ & Other Chemical and Allied Products Merchant Wholesalers & 164 \\
\hline $\mathbf{4 2 4 7 1 0}$ & Petroleum Bulk Stations and Terminals & 89 \\
\hline $\mathbf{4 2 4 9 1 0}$ & Farm Supplies Merchant Wholesalers & 6 \\
\hline $\mathbf{4 9 3 1 2 0}$ & Refrigerated Warehousing and Storage & 1 \\
\hline $\mathbf{5 6 1 4 9 9}$ & All Other Business Support Services & 1 \\
\hline $\mathbf{5 6 2 1 1 2}$ & Hazardous Waste Collection & 213 \\
\hline $\mathbf{5 6 2 2 1 1}$ & Hazardous Waste Treatment and Disposal & 372 \\
\hline $\mathbf{7 2 2 3 1 0}$ & Food Service Contractors & 1 \\
\hline
\end{tabular}

chemicals predicted for each industry. Although the 126 industries covered were just a little over $10 \%$ of the industrial possibilities, the range of industries and industrial processes was impressive, ranging from chemical manufacturing and processing, to food service industries, to furniture manufacturing. As an example, 34 industrial types were identified in the Vicksburg/Jackson area, and the TIC-Master industry list thoroughly encompassed these industries.

\section{Integrated Geographic Information System}

\section{Introduction}

Both the intelligence and emergency management communities rely on having accurate, timely, and authoritative sources of geospatial information for their respective missions (Haefner 2004, Channell et al. 2009). Geographic Information Systems (GIS) play a critical role for visualization, and spatial analysis of population, critical infrastructure, and potential impact area to help manage disasters, and share a common operational picture (COP) amongst the stakeholders.

The primary purpose of the TIC-GIS effort was to research methods to design a toxic industrial chemicals database linked to a GIS, develop a framework to share the TIC database for peer review, and receive feedback from the emergency management community. 
Objectives:

1. Develop a TIC data model and a data dictionary.

2. Develop a capability to query the TIC database on a map.

3. Demonstrate the value of a geographic information system through relevant spatial analysis and automated reporting to support emergency management.

\section{Methodology}

A relational TIC data model (Figure 2) was designed based on the tables containing the toxic industrial chemicals and relevant properties created for this project.

\begin{tabular}{|c|c|c|c|c|c|c|c|c|c|}
\hline \multicolumn{4}{|c|}{ Top 63 TICS } & \multicolumn{2}{|c|}{ Chemicain - Industries Crons Walk } & \multicolumn{4}{|c|}{ Industries } \\
\hline bo chemisine & WWERE & $(2.92$ & 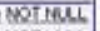 & Cherricallo & HTEOCR $\{7.0) \mathrm{MLL}$ & Sinduetryo & Mareak & $(2.02$ & Qormu: \\
\hline CASS D 0 & MUERE: & $(7,0)$ & NoT NeEL & industry D & Numeroc $(7,0) \mathrm{mLL}$ & Fusinese Mame & TEXT & (150) & NeL \\
\hline Mame & text & (100) & NOT NuLL & Chantical Name & TEXT (100) MULL. & Bunness Type & TEKT & (150) & nat. \\
\hline Suste & TEXT & & Not Nuel & CAS D & MUAFAC $(7,0)$ NOT MUL. & Otflee liame & TEXT & (150) & Mu \\
\hline Colo & TECT & (20) & Met & NAICS Thie & TEXT (2S5) MUL & Teleghore & IEXI & (12) & not meel \\
\hline Oder & TEXT & (20) & mol & Tripet Probseaty & Otcaeal $(6,2)$ mel & Enstoyeet & LONO WTEGER & & nese \\
\hline Secrage State & TEXT & (1) & weil & Naucs code & numere (6) lus. & Birect AddI & TEXT & (150) & nest. \\
\hline Metrmg Pont & FLOAT & & pare & 80 & BUPEAC (7) VOTMUL & Street Add2 & TEXT & (150) & wet \\
\hline Boling Pourt & FLOAT & & MeL & Benmarlingue & Key (D) & cty & IEXT & (100). & NoLL \\
\hline Vapor Densaty & DECRAAL. & $(6.2)$ & Nall & & & Stath & TEXT & (100) & Nuse \\
\hline Woter Solvbay & TEXT & (15) & mil & & & Zkxodh & Munerpic & $(10,0)$ & ) nous \\
\hline Exposure Rate & TLXT & (15) & mes & & & Lathide & DOUELE PFECSOON & & nes. \\
\hline IAC Faner Protection & TEXT & & mer & & & Longhude & DOUELE PFECTSOOA & & wet \\
\hline IEPAH & MAMEAC & $(1,0)$ & mal & & & Dota Seurce & TEXT & (100) & MUL \\
\hline IEPAF & MUERE & $(1,0)$ & Nall & & & Dutn Colncted & DATE & & ser. \\
\hline MPAJ & MUMEPRE & $(1,0)$ & ani & & & Aecuracy & TEXT & (100) & nas \\
\hline IFPA_Special & TEXT & (15) & Nou & & & SCCode & MUMEATC & (4) & whe \\
\hline HPV & BOCLEAN & & meil & & & SK Detcription & TEXT & (255) & ane \\
\hline IfF25 Jazardoun & TEXT & (6) & Nel & & & maks coove & MUAERA: & (6) & sase \\
\hline Ifl 40 Piorty & BOOLEAH & & mell & & & MALCS Descrighen & TEXT & (255) & mos \\
\hline IfF $40 \mathrm{HSC}$ & IEXT & (12) & wase & & & SHAPE & BELARY EY Y Y Y Y & & Net \\
\hline ITF $40 \mathrm{HPC}$ & TEXT & (12) & mele & & & Bg primary turique Ke & ey ondustry on & & \\
\hline ITF 40 FA & TEXT & (12) & nell & & & & & & \\
\hline ITr 40 Hist & TExt & (12) & MeL & & & & & & \\
\hline \multicolumn{4}{|c|}{ So Prinary / Unaque Key (Cnemica D) } & & & & & & \\
\hline
\end{tabular}

Figure 2. Relational TIC data model.

This model was developed using best industry practices for designing a normalized relational model to include a crosswalk table for handling the multiple relationships between the chemicals and industries tables, respectively.

A TIC database was created with the data model in an Environmental Systems Research Institute (ESRI) file geodatabase format. The ESRI file geodatabase format was selected for a rapid prototyping of a TIC database linked to a geographic information system. The data model enables a user to click a facility on a map and retrieve the list of chemicals likely to be found at that facility based on the facility type (Figure 3). 


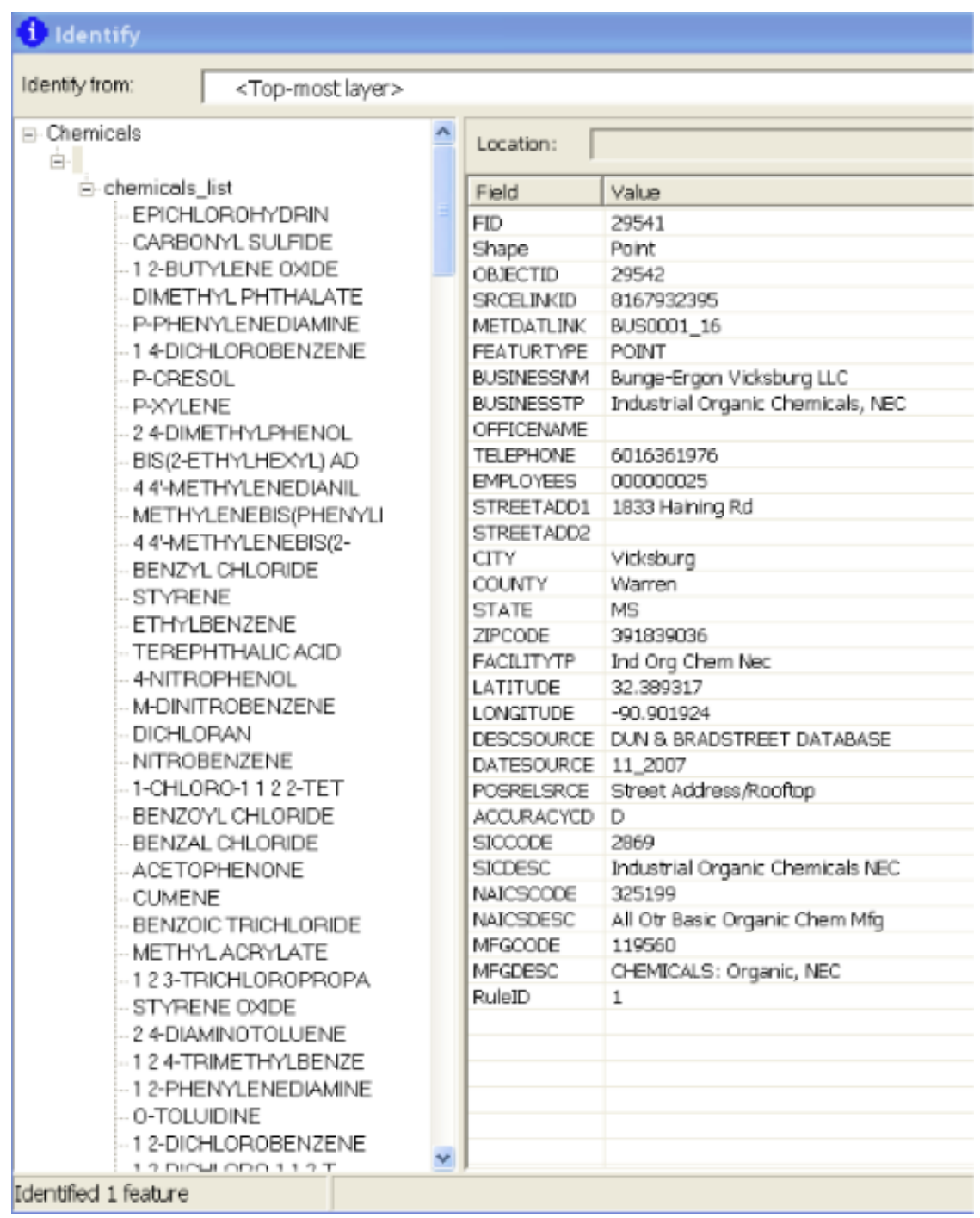

Figure 3. Pull-down screen showing facility type.

Users can click on a chemical to get its properties, as illustrated in Figure 4 for the chemical "phosgene."

ESRI software is part of the Commercial Joint Mapping Toolkit (C/JMTK) and a viable and completely compatible mechanism for transitioning capabilities to the Warfighter (Figure 5). A data dictionary was developed, containing tables, attributes, definitions of attributes, and attribute type.

A custom graphical user interface was designed in ARCGIS to demonstrate the value of linking a TIC database to an emergency management system developed around a geographic information system. The custom code was written in Visual Basic with the ESRI ARCOBJECTS library. The customized interface allows a user to navigate based on latitude/longitude, city, 


\begin{tabular}{|c|c|c|c|c|}
\hline \multicolumn{5}{|l|}{ (i) Identify } \\
\hline \multicolumn{5}{|c|}{$<$ Top-most layer > } \\
\hline \multirow{38}{*}{ : } & \multirow{38}{*}{ 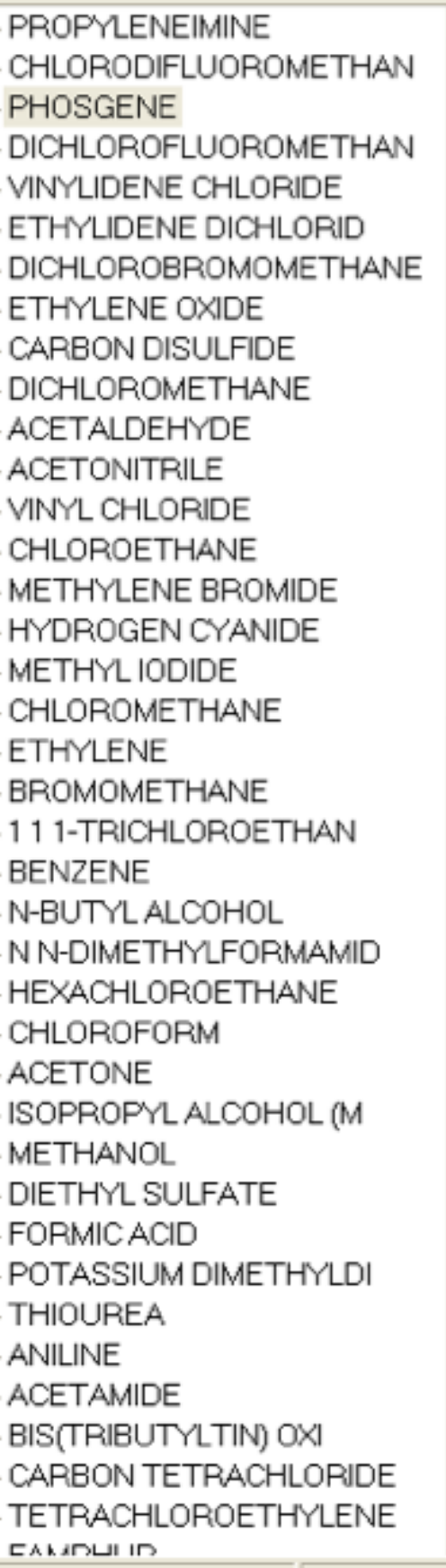 } & \multirow[t]{37}{*}{$\hat{-}$} & \multicolumn{2}{|l|}{ Location: } \\
\hline & & & Field & Value \\
\hline & & & OID & 38 \\
\hline & & & ICCW_ID & 39 \\
\hline & & & NAICS_TITL & All Other Basic Or \\
\hline & & & CAS & 75445 \\
\hline & & & TRICHEM_NA & PHOSGENE \\
\hline & & & TRI_PROB & 2.12 \\
\hline & & & NAICS & 325199 \\
\hline & & & OID_1 & 101 \\
\hline & & & CHEMICAL_I & 102 \\
\hline & & & CAS_1 & 75445 \\
\hline & & & CAS_TEXT & $75-44-5$ \\
\hline & & & CHEMICAL_N & phosgene \\
\hline & & & STATE & G \\
\hline & & & COLOR & no \\
\hline & & & ODOR & yes \\
\hline & & & STORAGE_ST & LG \\
\hline & & & MELTING_PO & -118 \\
\hline & & & BOILING_PO & 8 \\
\hline & & & VAPOR_DENS & 3 \\
\hline & & & WATER_SOLU & reaction \\
\hline & & & EXPOSURE_R & Inh \\
\hline & & & NBCFILTER_- & $\mathrm{E}$ \\
\hline & & & NFPA_H & 4 \\
\hline & & & NFPA_F & 0 \\
\hline & & & NFPA_I & 1 \\
\hline & & & NFPA_SPECI & \\
\hline & & & HPV & yes \\
\hline & & & ITF25_HAZA & high \\
\hline & & & ITF40_PRIO & yes \\
\hline & & & ITF40_HSC & Critical \\
\hline & & & ITF40_HPC & Likely \\
\hline & & & ITF40_RR & High \\
\hline & & & ITF40_HIST & Weapon \\
\hline & & & SPECIAL_CO & \\
\hline & & & & \\
\hline & & $\underline{v}$ & & \\
\hline \multicolumn{5}{|c|}{ Identified 1 feature } \\
\hline
\end{tabular}

Figure 4. Phosgene example. 


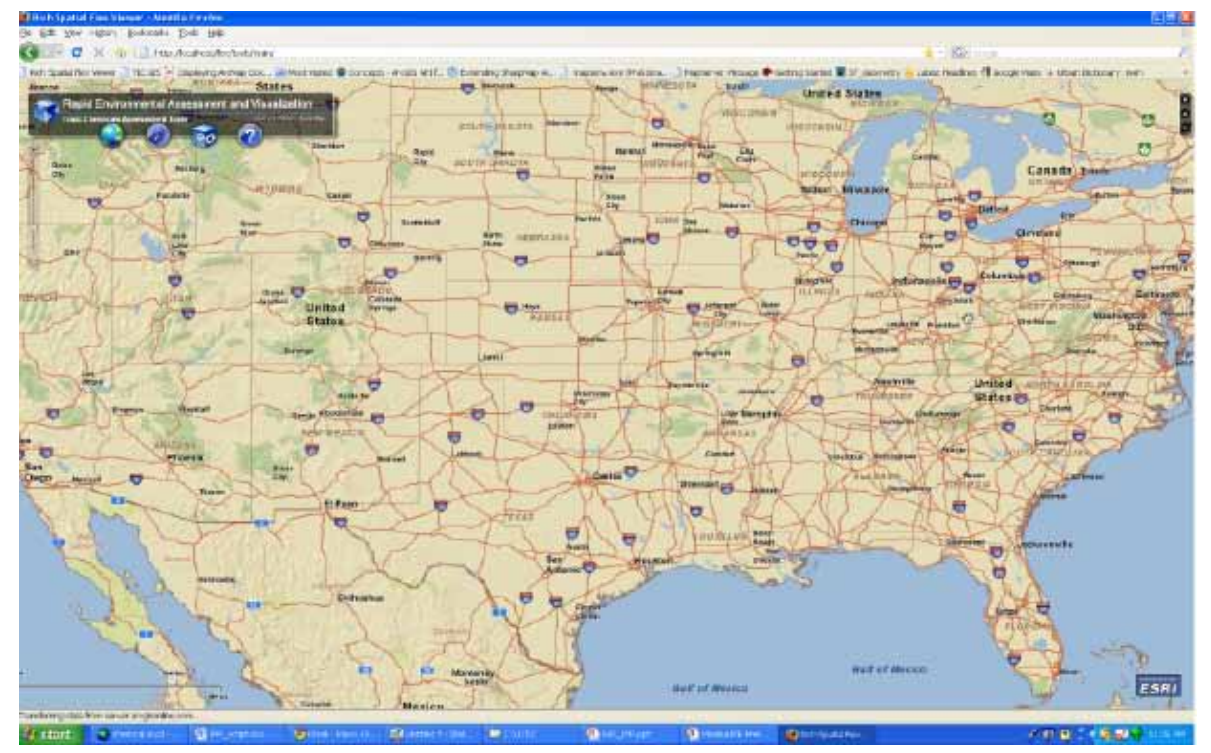

Figure 5. ESRI GIS interface.

state, and ZIP code in the continental United States (Figure 5). The GIS system could be applied to areas all over the world. However, some of the features were only available within the United States. Databases are available to apply most of these capabilities worldwide, but were not investigated as part of the scope for this project since it was developed as a scoping project to see how the concept would function using primarily free source data.

The system allowed for searches of key entities, such as fire and police departments and schools. The system also had the capability to search for facilities that use industrial chemicals within the United States (Figure 6 shows an example of facilities identified within the Warfighter III training area). For the system tested, this feature was only available within the United States (by using the "Homeland Security Infrastructure Protection Database" provided by the National Geospatial-Intelligence Agency); however, international databases exist that allow for similar capabilities around the world (such as the "Directory of World Chemical Producers"). In unstable areas where armed conflict occurs, industrial activity information may be inaccurate. However, most industries have international ties in terms of chemical and machinery sources and in terms of export agreements. Therefore, these records are probably fairly useful for most situations. A polygon feature allows for estimation of populations within a defined area based on census data (Figure 7). Once again, this feature is available with international database add-ons. 


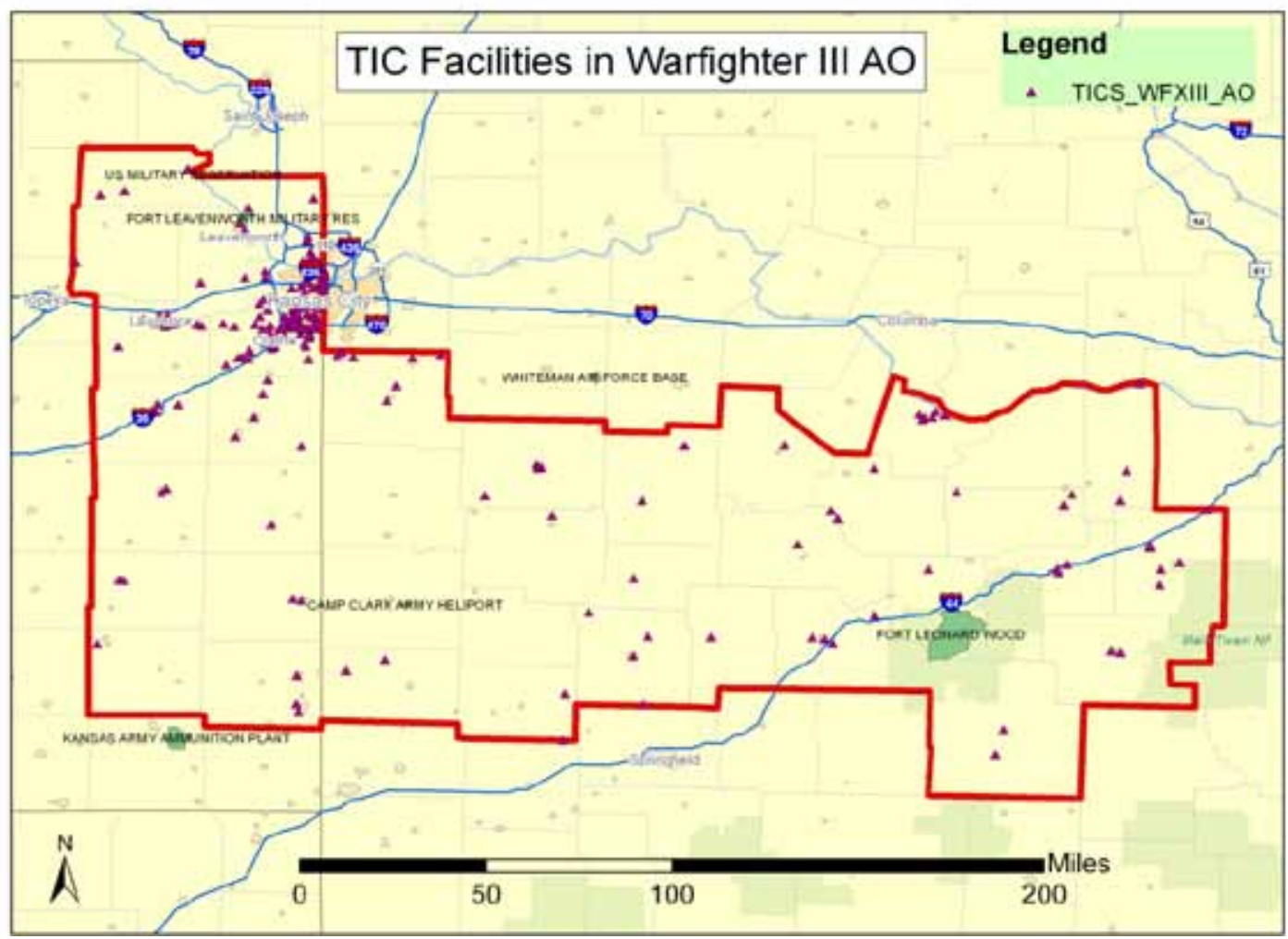

Figure 6. Location of toxic industrial chemical facilites in the 2009 Warfigher III test area.

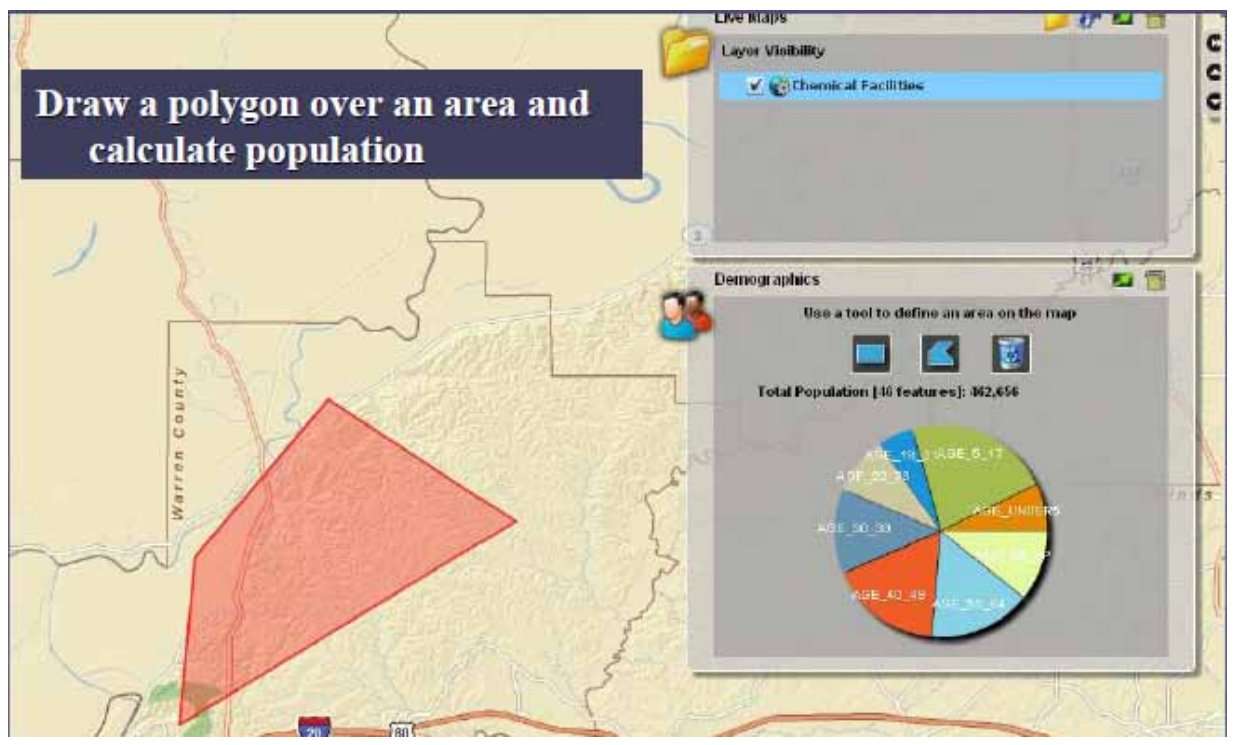

Figure 7. Demonstration of the polygon tool to obtain census data.

\section{Outreach}

An outreach activity was used to expose the TIC-Master Data Model to the Emergency Management Community through the Geospatial Acquisition Support Directorate of the Army Geospatial Center, and the Homeland 
Infrastructure Foundation Level Database Working Group (HIFLD-WG). Within the HIFLD-WG, outreach involved collaboration with Craig P. Adams from the Office of the Deputy Under Secretary of Defense (Installations and the Environment) (ODUSD(I\&E)). Mr. Adams is the Business Enterprise Integration Program Manager responsible for the central DoD chemicals database. This project provided value-added attributes, including the probability of a toxic industrial chemical to be found at a particular location based on the facility type. The Army Data Model Program Manager was contacted and utilized to transition the TIC data model into the Army Data Model.

\section{Release modeling using ALOHA (Areal Locations of Hazardous Atmospheres)}

ALOHA (Areal Locations of Hazardous Atmospheres) was selected as the means of release modeling for this project. On 27 May 2009, Dr. Victor Medina and Agnes Morrow interviewed representatives of the Vicksburg Fire Department (VFD) (Vicksburg, MS) on emergency response issues to guide the development of the TIC-Master. The VFD indicated that they use a number of release models, but that ALOHA is the one they rely on in most cases and that ALOHA is commonly used by other first responder organizations. ALOHA was jointly developed by the National Oceanic and Atmospheric Administration (NOAA) and the USEPA and is designed especially for use by emergency responders, as well as for emergency planning and training. ALOHA models key hazards (toxicity, flammability, thermal radiation (heat), and overpressure (explosion blast force)) related to chemical releases that result in toxic gas dispersions, fires, and/or explosions. ALOHA runs quickly on small, easily transportable computers (Microsoft $®$ Windows $($ ) or Macintosh $®$ ). It is designed to be easy to use so that it can be successfully operated during high-stress situations. Its chemical library contains information about the physical properties of approximately 1,000 common hazardous chemicals. Its computations represent a compromise between accuracy and speed; ALOHA has been designed to produce good results quickly enough to be of use to responders. ALOHA is also designed to minimize operator error as it checks entered information and warns the user when a mistake seems evident. ALOHA's on-screen help offers quick access to explanations of its features and computations, as well as background information to help in interpreting its output. Although ALOHA was the only program used in this study, other release modeling programs could easily be added to the GIS interface. 


\section{Standard operating procedures for environmental releases of selected chemicals}

A goal of the project was to provide detailed environmental response information for TICs - Standard Operating Procedures (SOPs). This process required a detailed literature review for each chemical. Based on the resources for the project, it was determined that it would not be possible to create an SOP for the $>2000$ chemicals in the database. So, the focus was narrowed to the 63 chemicals identified as most problematic in ITF-40.

Of these 63 chemicals, 35 existed only as a gas. Gaseous releases would likely have a minimal long-term environmental impact, so SOPs were not prepared for those materials. Therefore, SOPs were prepared for 28 chemicals. Figure 8 is an example of the SOP for acrolein. Appendix C contains the SOPs prepared for the project.

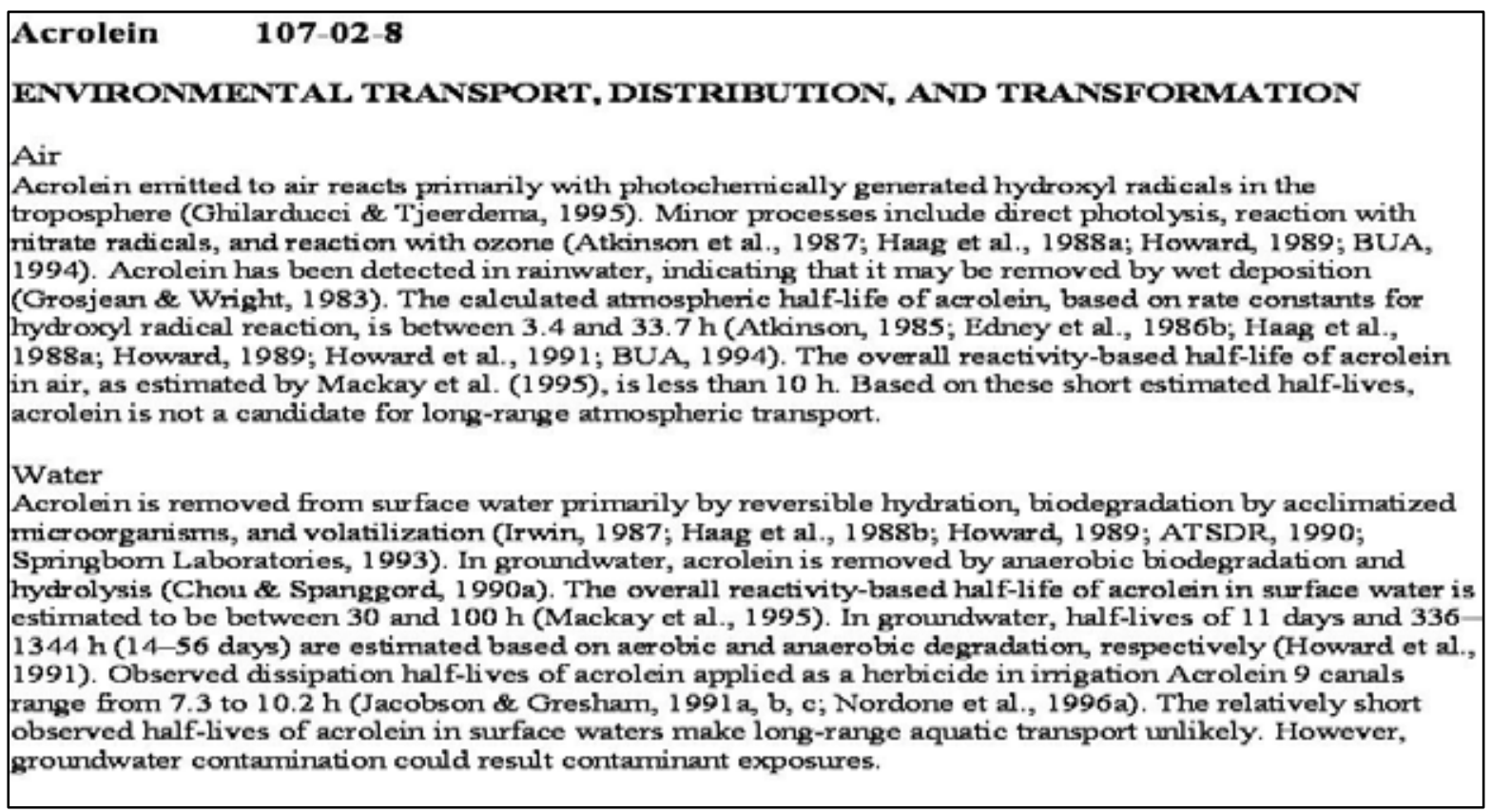

Figure 8. Example of part of an SOP for acrolein. 


\section{Testing of TIC-Master}

\section{Overview}

A testing program was developed to evaluate the TIC-Master program. Key aspects of the test addressed the following questions:

- How accurate was the TIC-Master at predicting specific chemicals from a given industrial process and at determining an overall chemical threat?

- Could TIC-Master prove useful in operational planning (particularly using release modeling)?

- How easy was the program to use?

- What could be improved?

A test team consisting of Dr. Dennis Brandon, Dr. Heather Smith, and Agnes Morrow, developed test scenarios and evaluated the results. This group was not involved in any way with the development of the TICMaster program. Nor were these team members involved with the TICMaster operation during testing. The TIC-Master operation was conducted by Dhiren Khona, Dr. James Hay, and Dr. Victor Medina. The Vicksburg/Jackson MS area was chosen as the area to test, primarily because it was convenient for the ERDC Vicksburg staff to obtain industrial information for this area.

\section{Scenario development}

Five scenarios were developed and submitted to the test team separately. Each was submitted using a standard form (Figure 9), which provided background information for the test team. The industrial process, TIC release mechanism (i.e., area secured after a fierce aerial assault, explosion due to unknown cause, suicide bombers drive vehicles into facility) and atmospheric conditions changed with each scenario. Four scenarios provided the industrial process or NAICS. The other scenarios provided a partial or complete list of chemicals. Several scenarios included multiple sites, other hazards, and additional site characteristics. The primary route of exposure alternated between air, water, and surface soil. The sites varied in their proximity to civilian populations, water bodies, and intrastructure (i.e., schools, hospitals, and historical landmarks). 


\begin{tabular}{|c|c|c|}
\hline \multicolumn{3}{|c|}{ Test Scenario } \\
\hline \multicolumn{3}{|c|}{$\begin{array}{l}\text { Date: } \quad \text { Time Submitted: } \\
\text { Team Initial Start Time: }\end{array}$} \\
\hline $\begin{array}{l}\text { Industrial Processes or } \\
\text { Process Name: }\end{array}$ & TIC Release Mechanism: & $\begin{array}{l}\text { Atmospheric Conditions: } \\
\text { Temperature: } \\
\text { Wind Speed: } \\
\text { Wind Direction: } \\
\text { Precipitation: }\end{array}$ \\
\hline Suspected TICs: & $\begin{array}{l}\text { Other Industrial } \\
\text { Chemicals: }\end{array}$ & Other Potential Hazards: \\
\hline $\begin{array}{l}\text { Site Designation: } \\
\text { Single Site: } \\
\text { Multiple Sites: } \\
\text { General Location*: }\end{array}$ & $\begin{array}{l}\text { Site A*: } \\
\text { Longitude: } \\
\text { Latitude: } \\
\text { Elevation: }\end{array}$ & $\begin{array}{l}\text { Site B: } \\
\text { Longitude: } \\
\text { Latitude: } \\
\text { Elevation: }\end{array}$ \\
\hline Other Characteristics: & & \\
\hline $\begin{array}{l}\text { * See the Other Charact } \\
\text { occupy a } 2 \text {-square-mile }\end{array}$ & $\begin{array}{l}\text { ics section. For example, } t \\
\text { centered at the location } p\end{array}$ & $\begin{array}{l}\text { 1e U.S. Army may wish to } \\
\text { ovided by Site A*. }\end{array}$ \\
\hline
\end{tabular}

Figure 9. Test scenario submission form.

The selected facilities were in the vicinity of Jackson/Vicksburg, MS (Table 4). Copies of 2008 EPA TIER 2 reports were obtained from the Mississippi Emergency Management Agency (MEMA). This request was based on the Freedom of Information Act. The evaluation team used these reports to validate the site chemical lists provided by the test team. 
Table 4. List of facilities used for TIC-Master testing.

\begin{tabular}{|l|l|l|}
\hline Name & NAICS & Location \\
\hline AKZO Nobel Coatings Inc. & 325510 & Clinton, MS \\
\hline Bunge-Ergon Vicksburg, LLC & 311222 & Vicksburg, MS \\
\hline $\begin{array}{l}\text { Clorox Products } \\
\text { Manufacturing Co. }\end{array}$ & 325612 & Pearl, MS \\
\hline Double G Coatings Co., L.P. & 332813 & Jackson, MS \\
\hline Ergon Refining, Inc. & 324110 & Vicksburg, MS \\
\hline Georgia Gulf Corp. and Vinyls & 325991 & Hazelhurst, MS \\
\hline International Paper & 322121 & Redwood, MS \\
\hline Jackson Plating Co. & 332813 & Jackson, MS \\
\hline Nissan North America & $336111 / 336112$ & Canton, MS \\
\hline
\end{tabular}




\section{Test Results}

\section{Test 1}

\section{Scenario}

The scenario assumes an explosion has occurred and remnants of the subsequent fire remain. The NAICS code was 325510. Figure 10 is the Test 1 scenario description form.

\section{Test__ Scenario}

Date:10/09/09 Time Submitted:_0830 CDT Time Team Response Received: 09:00

Team Initial Start Time:

We worked on the test from 09:45 to 10:45, then from 13:15 to 14:45. total time 3 hours

\begin{tabular}{l|l|l}
\hline Industrial Processes or Process & TIC Release Mechanism: & Atmospheric Conditions:
\end{tabular}

Name:

NAICS: 325510

An explosion has occurred. Temperature: $79^{\circ} \mathrm{F}$

Remnants of the subsequent

Wind Speed: $20 \mathrm{mph}$

Suspected TICs:

Suspected TICs: fire remain.

Wind Direction: NE

Precipitation: None

\begin{tabular}{|l|l|l|}
\hline Suspected TICs: & Other Industrial Chemicals: & Other Potential Hazards: \\
Flammable liquids are on site. \\
\hline $\begin{array}{l}\text { Site Designation: } \\
\begin{array}{l}\text { Multiple Sites: } \\
\text { General Location*: }\end{array}\end{array}$ & $\begin{array}{l}\text { Site A*: } \\
\text { Longitude: } 90^{\circ} 21^{\prime} 47.16^{\prime \prime} \text { W W } \\
\text { Latitude: } 32^{\circ} 21^{\prime} 02.57^{\prime} \mathrm{N} \\
\text { Elevation: }\end{array}$ & $\begin{array}{l}\text { Longitude: } \\
\text { Latitude: } \\
\text { Elevation: }\end{array}$ \\
\hline$\underline{\text { Other Characteristics: }}$ & \begin{tabular}{l} 
Site B: \\
\hline
\end{tabular} & \\
\hline
\end{tabular}

* See the Other Characteristics section. For example, the U.S. Army may wish to occupy a 2square-mile area centered at the location provided by Site $A^{*}$. 


\section{Results}

Response time

The total response time was $3.5 \mathrm{hr}$, above the 2.0-hr goal. The challenge came from sorting out chemical hazards, which was done manually for this test.

\section{Facility location and identification}

Figure 11 is a location map of the facility based on the GIS coordinates. The facility was correctly identified as a painting and coatings manufacturing facility. The actual facility was AKZO Nobel Coatings.

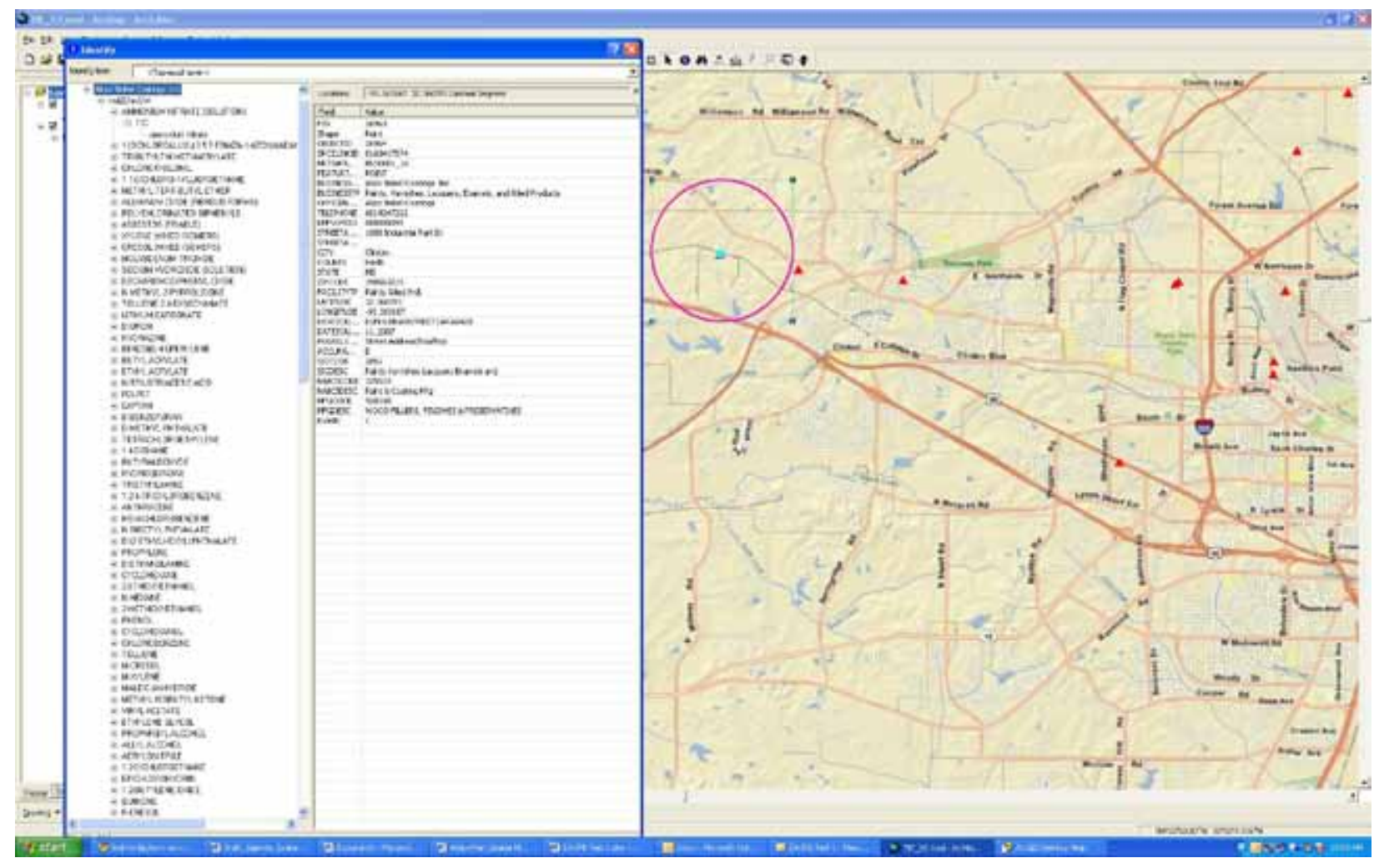

Figure 11. Location map for facility identified in Test 1.

\section{Chemical identification}

The 2009 Tier II report (reporting from 1 January to 31 December 2008) submitted by AKZO Nobel Coatings Inc. was used to verify the chemicals identified by the test team. The test team identified 156 TICs (Table 5). 
Table 5. Chemical identification list for Test 1 scenario. CAS is the chemical abstracts services number for the chemical.

\begin{tabular}{|c|c|c|c|}
\hline CAS & Chemical Name & CAS & Chemical Name \\
\hline 50000 & FORMALDEHYDE & 80626 & METHYL METHACRYLATE \\
\hline 56235 & CARBON TETRACHLORIDE & 84662 & DIETHYL PHTHALATE \\
\hline 56359 & BIS(TRIBUTYLTIN) OXIDE & 84742 & DIBUTYL PHTHALATE \\
\hline 57749 & CHLORDANE & 85018 & PHENANTHRENE \\
\hline 64186 & FORMIC ACID & 85449 & PHTHALIC ANHYDRIDE \\
\hline 64675 & DIETHYL SULFATE & 85687 & BUTYL BENZYL PHTHALATE \\
\hline 67561 & METHANOL & 91087 & TOLUENE-2 6-DIISOCYANATE \\
\hline 67630 & ISOPROPYL ALCOHOL & 91203 & NAPHTHALENE \\
\hline 67641 & ACETONE & 92524 & BIPHENYL \\
\hline 68122 & N N-DIMETHYLFORMAMIDE & 94360 & BENZOYL PEROXIDE \\
\hline 71363 & N-BUTYL ALCOHOL & 95476 & O-XYLENE \\
\hline 71432 & BENZENE & 95487 & O-CRESOL \\
\hline 71556 & 11 1-TRICHLOROETHANE & 95501 & 1 2-DICHLOROBENZENE \\
\hline 74873 & CHLOROMETHANE & 95636 & 12 4-TRIMETHYLBENZENE \\
\hline 75058 & ACETONITRILE & 96333 & METHYL ACRYLATE \\
\hline 75092 & DICHLOROMETHANE & 98828 & CUMENE \\
\hline 75354 & VINYLIDENE CHLORIDE & 98884 & BENZOYL CHLORIDE \\
\hline 75456 & CHLORODIFLUOROMETHANE & 98953 & NITROBENZENE \\
\hline 75558 & PROPYLENEIMINE & 100210 & TEREPHTHALIC ACID \\
\hline 75569 & PROPYLENE OXIDE & 100414 & ETHYLBENZENE \\
\hline 75650 & TERT-BUTYL ALCOHOL & 100425 & STYRENE \\
\hline 75694 & TRICHLOROFLUOROMETHANE & 100447 & BENZYL CHLORIDE \\
\hline 75718 & DICHLORODIFLUOROMETHANE & 101144 & 4 4'-METHYLENEBIS(2-CHLOROANILINE) \\
\hline 76131 & FREON 113 & 101688 & METHYLENEBIS(PHENYLISOCYANATE) \\
\hline 77736 & DICYCLOPENTADIENE & 101779 & 4 4'-METHYLENEDIANILINE \\
\hline 78922 & SEC-BUTYL ALCOHOL & 101906 & DIGLYCIDYL RESORCINOL ETHER \\
\hline 78933 & METHYL ETHYL KETONE & 103231 & BIS(2-ETHYLHEXYL) ADIPATE \\
\hline 79005 & 11 2-TRICHLOROETHANE & 105679 & 2 4-DIMETHYLPHENOL \\
\hline 79016 & TRICHLOROETHYLENE & 106423 & P-XYLENE \\
\hline 79061 & ACRYLAMIDE & 106445 & P-CRESOL \\
\hline 79107 & ACRYLIC ACID & 106514 & QUINONE \\
\hline 79469 & 2-NITROPROPANE & 106887 & 12-BUTYLENE OXIDE \\
\hline 79947 & TETRABROMOBISPHENOL A & 106898 & EPICHLOROHYDRIN \\
\hline 80057 & 4 4'-ISOPROPYLIDENEDIPHENOL & 107062 & 1 2-DICHLOROETHANE \\
\hline 80159 & CUMENE HYDROPEROXIDE & 107131 & ACRYLONITRILE \\
\hline
\end{tabular}




\begin{tabular}{|c|c|c|c|}
\hline CAS & Chemical Name & CAS & Chemical Name \\
\hline 107186 & ALLYL ALCOHOL & 302012 & HYDRAZINE \\
\hline 107197 & PROPARGYL ALCOHOL & 330541 & DIURON \\
\hline 107211 & ETHYLENE GLYCOL & 554132 & LITHIUM CARBONATE \\
\hline 108054 & VINYL ACETATE & 584849 & TOLUENE-2 4-DIISOCYANATE \\
\hline 108101 & METHYL ISOBUTYL KETONE & 872504 & N-METHYL-2-PYRROLIDONE \\
\hline 108316 & MALEIC ANHYDRIDE & 1163195 & DECABROMODIPHENYL OXIDE \\
\hline 108383 & M-XYLENE & 1310732 & SODIUM HYDROXIDE (SOLUTION) \\
\hline 108394 & M-CRESOL & 1313275 & MOLYBDENUM TRIOXIDE \\
\hline 108883 & TOLUENE & 1319773 & CRESOL (MIXED ISOMERS) \\
\hline 108907 & CHLOROBENZENE & 1330207 & XYLENE (MIXED ISOMERS) \\
\hline 108930 & CYCLOHEXANOL & 1332214 & ASBESTOS (FRIABLE) \\
\hline 108952 & PHENOL & 1336363 & POLYCHLORINATED BIPHENYLS \\
\hline 109864 & 2-METHOXYETHANOL & 1344281 & ALUMINUM OXIDE (FIBROUS FORMS) \\
\hline 110543 & N-HEXANE & 1634044 & METHYL TERT-BUTYL ETHER \\
\hline 110805 & 2-ETHOXYETHANOL & 1717006 & 1 1-DICHLORO-1-FLUOROETHANE \\
\hline 110827 & CYCLOHEXANE & 1897456 & CHLOROTHALONIL \\
\hline 111422 & DIETHANOLAMINE & 2155706 & TRIBUTYLTIN METHACRYLATE \\
\hline 115071 & PROPYLENE & 4080313 & $\begin{array}{l}\text { 1-(3-CHLOROALLYL)-3 } 5 \text { 7-TRIAZA-1- } \\
\text { AZONIAADAMANTANE CHLORIDE }\end{array}$ \\
\hline 117817 & DI(2-ETHYLHEXYL) PHTHALATE & 6484522 & AMMONIUM NITRATE (SOLUTION) \\
\hline 117840 & N-DIOCTYL PHTHALATE & 7429905 & ALUMINUM (FUME OR DUST) \\
\hline 118741 & HEXACHLOROBENZENE & 7439921 & LEAD \\
\hline 120127 & ANTHRACENE & 7439965 & MANGANESE \\
\hline 120821 & 12 4-TRICHLOROBENZENE & 7439976 & MERCURY \\
\hline 121448 & TRIETHYLAMINE & 7440020 & NICKEL \\
\hline 123319 & HYDROQUINONE & 7440224 & SILVER \\
\hline 123728 & BUTYRALDEHYDE & 7440360 & ANTIMONY \\
\hline 123911 & 14 -DIOXANE & 7440393 & BARIUM \\
\hline 127184 & TETRACHLOROETHYLENE & 7440439 & CADMIUM \\
\hline 131113 & DIMETHYL PHTHALATE & 7440473 & CHROMIUM \\
\hline 132649 & DIBENZOFURAN & 7440484 & COBALT \\
\hline 133062 & CAPTAN & 7440508 & COPPER \\
\hline 133073 & FOLPET & 7440666 & ZINC (FUME OR DUST) \\
\hline 139139 & NITRILOTRIACETIC ACID & 7632000 & SODIUM NITRITE \\
\hline 140885 & ETHYL ACRYLATE & 7647010 & $\begin{array}{l}\text { HYDROCHLORIC ACID (1995 AND AFTER } \\
\text { "ACID AEROSOLS" ONLY) }\end{array}$ \\
\hline 141322 & BUTYL ACRYLATE & 7664382 & PHOSPHORIC ACID \\
\hline 191242 & BENZO(G H I)PERYLENE & 7664393 & HYDROGEN FLUORIDE \\
\hline
\end{tabular}




\begin{tabular}{|l|l||l|l|}
\hline CAS & Chemical Name & CAS & Chemical Name \\
\hline 7664417 & AMMONIA & 12122677 & ZINEB \\
\hline 7664939 & $\begin{array}{l}\text { SULFURIC ACID (1994 AND AFTER "ACID } \\
\text { AEROSOLS" ONLY) }\end{array}$ & 20325400 & $\begin{array}{l}\text { 3 3'-DIMETHOXYBENZIDINE } \\
\text { DIHYDROCHLORIDE }\end{array}$ \\
\hline 7697372 & NITRIC ACID & 25321226 & DICHLOROBENZENE (MIXED ISOMERS) \\
\hline 7726956 & BROMINE & 26471625 & $\begin{array}{l}\text { TOLUENE DIISOCYANATE (MIXED } \\
\text { ISOMERS) }\end{array}$ \\
\hline 7782492 & SELENIUM & 35691657 & $\begin{array}{l}\text { 1-BROMO-1-(BROMOMETHYL)-13- } \\
\text { PROPANEDICARBONITRILE }\end{array}$ \\
\hline 7782505 & CHLORINE & 55406536 & 3-IODO-2-PROPYNYL BUTYLCARBAMATE \\
\hline 10294345 & BORON TRICHLORIDE & 60207901 & PROPICONAZOLE \\
\hline
\end{tabular}

The Tier II report listed 58 chemicals, but of these, only 35 were part of the database used for the project. The test team identified 15 of the 35 chemicals in the database (Table 6). The 20 remaining chemicals are listed in Table 7. Furthermore, the test team identified chemicals similar to those found at the site, including at least one chemical in the following classes: alkane, aldehyde, alcohol, ketone, aromatic, amide, and inorganic metals.

Table 6. Chemicals properly identified in Test 1.

\begin{tabular}{|l|l|l|}
\hline CAS \# & Chemical & Reported Max Daily Quantity (Ibs) \\
\hline 50000 & FORMALDEHYDE & $1,000-9,999$ \\
\hline 67561 & METHANOL & $10,000-99,999$ \\
\hline 67630 & ISOPROPYL ALCOHOL & $100,000-999,999$ \\
\hline 67641 & ACETONE & $100,000-999,999$ \\
\hline 71363 & N-BUTYL ALCOHOL & $10,000-99,999$ \\
\hline 78933 & METHYL ETHYL KETONE & $10,000-99,999$ \\
\hline 95636 & $124-T R I M E T H Y L B E N Z E N E$ & $10,000-99,999$ \\
\hline 100414 & ETHYLBENZENE & $10,000-99,999$ \\
\hline 108101 & METHYL ISOBUTYL KETONE & $10,000-99,999$ \\
\hline 108883 & TOLUENE & $10,000-99,999$ \\
\hline 108952 & PHENOL & $0-99$ \\
\hline 121448 & TRIETHYLAMINE & $1,000-9,999$ \\
\hline 1330207 & XYLENE (MIXED ISOMERS) & $10,000-99,999$ \\
\hline 7429905 & ALUMINUM (FUME OR DUST) & $1,000-9,999$ \\
\hline 7664417 & AMMONIA & $1,000-9,999$ \\
\hline
\end{tabular}


Table 7. Chemicals not precisely identified by the TIC-Master process for Test 1.

\begin{tabular}{|l|l|l|l|l|}
\hline CAS No. & Chemical & NFPA & HSC & Reported max daily quantity (Ibs) \\
\hline $64-17-5$ & Ethyl alcohol & H2, F3 & Critical & $10,000-99,999$ \\
\hline $78-83-1$ & Isobutanol & H2, F3 & Critical & $10,000-99,999$ \\
\hline $97-85-8$ & isobutyl isobutyrate & H1, F3 & Critical & $1,000-9,999$ \\
\hline $98-56-6$ & Parachlorobenzofluoride & H1 & Negligible & $10,000-99,999$ \\
\hline $104-15-4$ & p-toluene sulfonic acid & H2, F1 & Marginal & $10,000-99,999$ \\
\hline $108-21-4$ & Isopropyl acetate & H1, F3 & Critical & $1,000-9,999$ \\
\hline $108-65-6$ & 1-methoxy-2-acetolypropane & H1, F2 & Marginal & $10,000-99,999$ \\
\hline $110-19-0$ & Isobutl acetate & H2, F3 & Critical & $10,000-99,999$ \\
\hline $111-76-2$ & Butoxyethanol & H3, F2 & Critical & $10,000-99,999$ \\
\hline $110-43-0$ & Heptanone & H4, F2 & Catastrophic & $10,000-99,999$ \\
\hline $123-86-4$ & Butyl acetate & H2, F3 & Critical & $10,000-99,999$ \\
\hline $763-69-9$ & Ethyl-3-ethoxyproprionate & H1 & Negligible & $10,000-99,999$ \\
\hline $1314-13-2$ & Zinc oxide & H2 & Marginal & $10,000-99,999$ \\
\hline $1314-98-3$ & Zinc sulfide & & & $10,000-99,999$ \\
\hline $1333-86-4$ & Carbon black & H2 & Marginal & $10,000-99,999$ \\
\hline $8052-41-3$ & Petroleum hydrocarbon & H2, F2 & Marginal & $10,000-99,999$ \\
\hline $21645-51-2$ & Aluminum trihydroxide & & Indeterminant & $10,000-99,999$ \\
\hline $34590-94-8$ & Methoxtmethyl ethoxypropanol & H2, F2 & Marginal & $1,000-9,999$ \\
\hline $64742-89-8$ & Aliphatic hydrocarbon & & Indeterminate & $10,000-99,999$ \\
\hline $42978-66-5$ & Tripopylene glycol diacrylated & H1 & Marginal & $1,000-9,999$ \\
\hline
\end{tabular}

\section{Most probable chemicals}

The system allowed for ranking the probability of chemicals that would be found. The top ten chemicals are given in Table 8. Eight of these top ten chemicals were actually found in the inventory, including the first six chemicals on this list.

\section{Most significant chemical hazard}

Chlormethane, benzene, and styrene were the most significant hazards identified by the test team. This recommendation was based on the assumption that flammability was the greatest hazard. None of these chemicals were reported in the AKZO Tier II report. However, as these chemicals had flammability hazard indexes of 3 and 4 , their effect would be well within flammable hazards from other chemicals at the site. 
Table 8. The 10 most probable chemicals predicted by the TIC-Master database for Test 1. Chemicals found in the actual inventory are starred.

\begin{tabular}{|l|l|l|l|}
\hline \%Probability & CAS & NAME & Rank \\
\hline 65.41 & 1330207 & XYLENE (MIXED ISOMERS)* & 1 \\
\hline 56.16 & 108883 & TOLUENE* & 2 \\
\hline 47.51 & 78933 & METHYL ETHYL KETONE* & 3 \\
\hline 35.81 & 108101 & METHYL ISOBUTYL KETONE* & 4 \\
\hline 32.66 & 71363 & N-BUTYL ALCOHOL* & 5 \\
\hline 31.88 & 100414 & ETHYLBENZENE* & 6 \\
\hline 29.17 & 107211 & ETHYLENE GLYCOL & 7 \\
\hline 26.46 & 67641 & ACETONE* & 8 \\
\hline 25.15 & 95636 & 12 4-TRIMETHYLBENZENE & 9 \\
\hline 23.32 & 67561 & METHANOL* & 10 \\
\hline
\end{tabular}

There are, however, other ways to rank the most significant chemical hazards. HSC is a hazard severity ranking based on the ITF 40's method. Table 9 summarizes the chemicals identified by the TIC-Master program and found at the site. It is clear that there are several chemicals that could be severe chemical hazards.

Table 9. Evaluation of TICs in Test 1 for health hazards.

\begin{tabular}{|l|l|l|l|l|}
\hline CAS No. & Chemical & NFPA & HSC & Reported max daily quantity (Ibs) \\
\hline $110-43-0$ & 2 heptanone & H-4, F-2 & Catastrophic & $10,000-99,999$ \\
\hline $108-95-2$ & Phenol & H-4, F-2 & Catastrophic & $0-99$ \\
\hline $111-76-2$ & 2 butoxyethanol & H-3, F-3 & Critical & $10,000-99,999$ \\
\hline $67-56-1$ & Methyl alcohol & H-3, F-3 & Critical & $10,000-99,999$ \\
\hline $108-88-3$ & Toluene & H-3, F-3 & Critical & $10,000-99,999$ \\
\hline $1330-20-7$ & Xylene & H-3 & Critical & $10,000-99,999$ \\
\hline $121-44-8$ & Triethylamine & H-3, F-3 & Critical & $1,000-9,999$ \\
\hline
\end{tabular}

NFPA: National Fire Protection Association.

$\mathrm{H}$ : Health Hazard Rating (0 to 4 Scale). F: Flammability Hazard Rating (0 to 4 Scale).

HSC: ITF-40 Hazard Severity Classification.

\section{Release modeling}

Figure 12 is a map identifying the effects of a flammable release of xylene using ALOHA. The estimation was based on a reportable limit of $500 \mathrm{~kg}$. The TIC-Master program does not have the ability to specify storage vessels. 
Presumably, it would be possible for military intelligence to obtain storage vessel information. However, that was not provided in this case. ALOHA does, however, list likely storage vessels depending on the contaminant. This feature, as well as information on the density of xylene, was used to estimate that xylene was stored in a 200-gallon spherical tank. The explosive release included the weather conditions given in the start conditions.

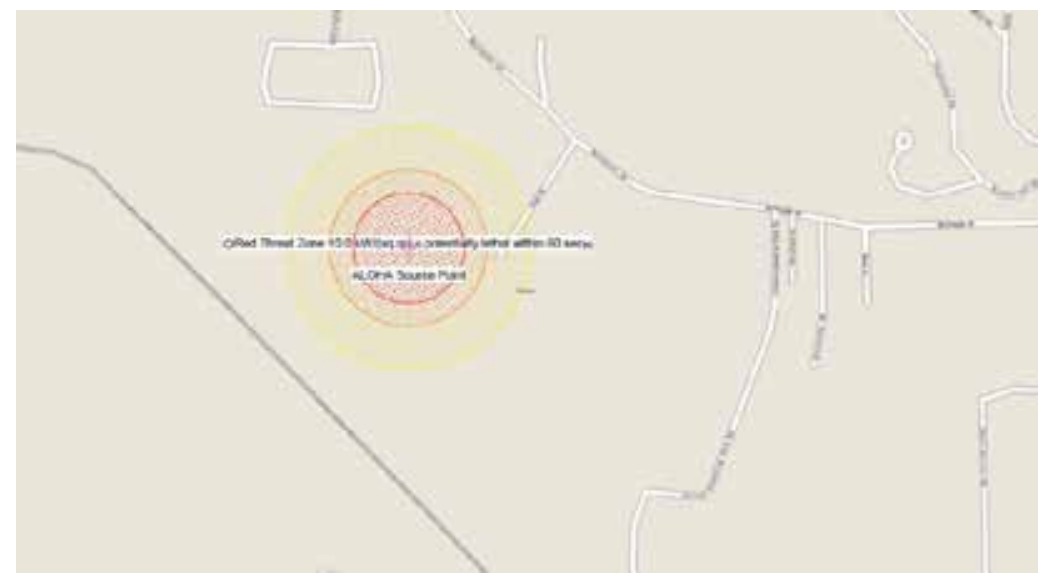

Figure 12. Release modeling of an explosion of a 200-gal tank of xylene. The innermost red circle indicates lethality within 60 seconds, and was about $\mathbf{2 0 0} \mathrm{m}$ in diameter. The circular pattern is common for explosions.

As seen, the explosive release from a circular tank creates a circular area of influence that is relatively uninfluenced by the wind. The zone of impact was about $500 \mathrm{~m}$ in diameter, with a $200-\mathrm{m}$ zone indicating lethality within 60 seconds. It should be possible to model secondary contaminant release following the explosion, if that is needed; however, it would be reasonable to assume that the contaminants would be largely consumed in the explosion.

Evaluation of chemical risks from ITF-40 63 most toxic chemicals

Three chemicals were on the SOP list: Acrylonitrile, formaldehyde, and propylene oxide.

\section{Acrylonitrile}

Fate - Biodegrades in soil.

Mitigation - Limit movement in soil and surface water and promote soil biodegradation. 


\section{Formaldehyde}

$\underline{\text { Risks }}$ - Because it readily biodegrades in soil, the primary issue is air. May also contaminate water $(\mathrm{MCL}=0.7 \mathrm{ug} / \mathrm{L})$.

Mitigation - Promote biodegradation in soil and groundwater. Waterbased sprays for air and absorbents for soil spills.

\section{Propylene oxide}

Risks - Photochemically reactive in atmosphere. Doesn't readily sorb to soil, so may migrate, but also undergoes hydrolysis reaction (reaction with water).

Mitigation - Limit spread in soils and water. Use water mists to remove from air.

\section{Issues}

Several issues were identified in this first test. First, it was hoped that all test team members would have access to the program, as the Army has a general license to ArcGIS. However, the license covers only the Reader version of the program, not the Editor version. The Reader version alone would not run the program, which limited the GIS portion of the work to Dhiren Khona.

Initially there were problems with automating the process of ranking the most probable contaminants. However, once this process was straightened out, the ranking system was found to be very accurate.

Ranking of the most hazardous materials was challenging. Initially, there was no search mechanism, which required manually searching the database for hazard information. Fortunately, this deficiency was rectified. Choosing criteria for hazard ranking would prove to be more challenging. Flammability and reactivity could be critical for certain applications. However, health and toxicity are also crucial. Issues regarding factors such as the likelihood of the presence or potential quantity of hazardous materials would continue to be dealt with throughout subsequent tests. 


\section{Test 2 Summary}

\section{Scenario}

A fire has occurred at a soybean processing facility. Six inches of rain has fallen within the last $8 \mathrm{hr}$. There are other flammable liquids on site. The scenario form is given as Figure 13.

\section{Test_ 2 _ Scenario}

Date: $\underline{10 / 16 / 09}$ Time Submitted:_0915 CDT Time Team Response Received:_09:30

Team Initial Start Time:_09:45__ Time Team Response Submitted:_14:15

Completed: 11:20

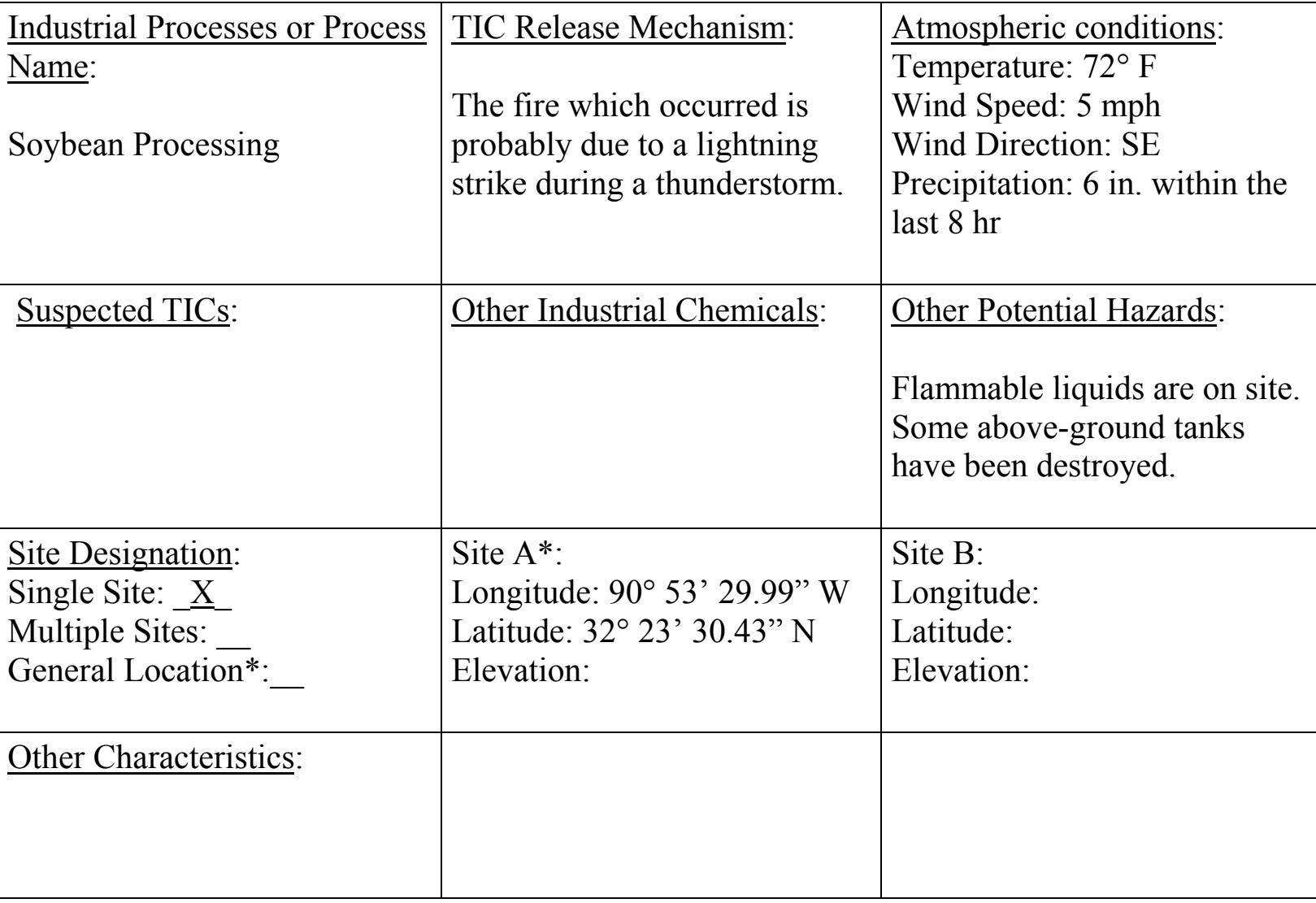

* See the Other Characteristics section. For example, the U.S. Army may wish to occupy a 2-square-mile area centered at the location provided by Site A*.

Figure 13. Scenario description for Test 2. 


\section{Results}

Response time

The response time was $1 \mathrm{hr} 35$ minutes. This response time is consistent with the test team's goal of providing a response within $2 \mathrm{hr}$.

\section{Facility location and identification}

Figure 14 is a location map for the facility. The facility and code NAICS 311222 were correctly identified as soybean processing. The actual facility was the Bunge-Ergon ethanol production facility in Vicksburg, MS.

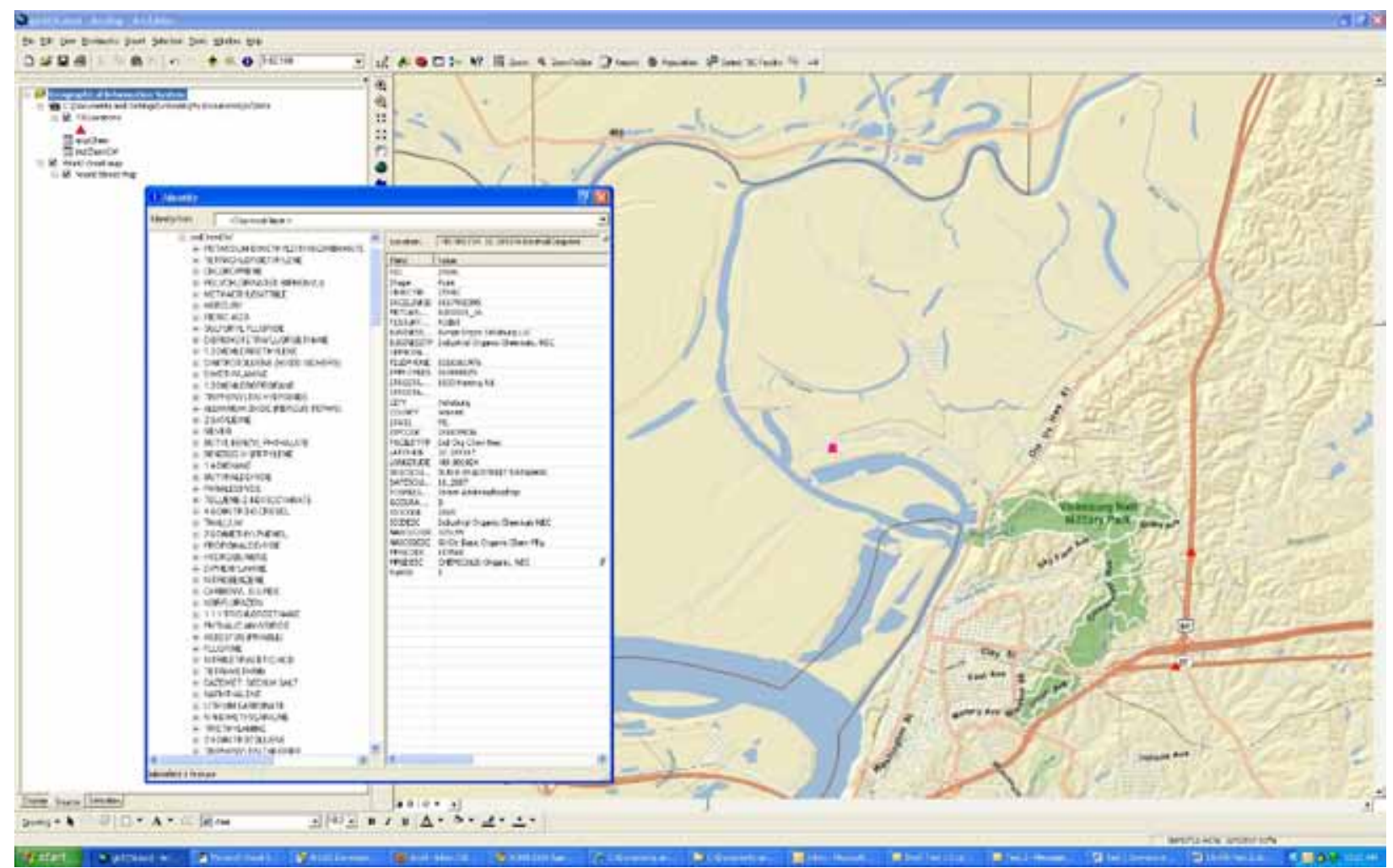

Figure 14. Location map for Test 2. The pink dot is the location of the facility - in the Port of Vicksburg.

Chemical identification

The database identified 26 TICs (Table 10). These included hydrocarbons, alcohols, ketones, metals, and toxic gases.

The 2009 Tier II report (reporting from 1 January to 31 December 2008) submitted by Bunge-Ergon Vicksburg, LLC was used to verify the chemicals identified by the test database. This report listed five chemicals, four of which were in the test database. The database correctly identified two of 
Table 10. Estimated chemical list for Test 2.

\begin{tabular}{|l|}
\hline CHEMICAL \\
\hline BENZO(G H I)PERYLENE \\
\hline N-HEXANE \\
\hline LEAD \\
\hline MERCURY \\
\hline SULFURIC ACID \\
\hline SODIUM HYDROXIDE (SOLUTION) \\
\hline PHOSPHORIC ACID \\
\hline METHANOL \\
\hline ACETONE \\
\hline ZINC (FUME OR DUST) \\
\hline NITRIC ACID \\
\hline HYDROCHLORIC ACID \\
\hline NICKEL \\
\hline CHLORINE \\
\hline AMMONIA \\
\hline NAPHTHALENE \\
\hline BIPHENYL \\
\hline PROPYLENE \\
\hline ASBESTOS (FRIABLE) \\
\hline POLYCHLORINATED BIPHENYLS \\
\hline MANGANESE \\
\hline ARSENIC \\
\hline COPPER \\
\hline SODIUM NITRITE \\
\hline SELENIUM \\
\hline AMMONIUM SULFATE (SOLUTION) \\
\hline
\end{tabular}

these four chemicals (Table 11). Of the remaining two chemicals, the database identified very similar chemicals - ammonia vs. aqueous ammonia and methanol vs. ethanol (Table 12).

Methanol and ethanol have very similar hazard characteristics. However, comparing ammonia to aqueous ammonia reveals some subtle, but possibly important, differences in hazard assessment, with the aqueous ammonia having a greater effect in terms of health, but the gaseous form having a slight flammability. 
Table 11. Correctly identified chemicals for Test 2 simulation.

\begin{tabular}{|l|l|l|l|}
\hline CAS & Chemical Name & Inventory (Max Daily Ibs) & Inventory (Ave Daily Ibs) \\
\hline $7664-93-9$ & Sulfuric Acid & $100,000-999,999$ & $10,000-99,999$ \\
\hline $1310-73-2$ & Sodium Hydroxide & $100,000-999,999$ & $100,000-999,999$ \\
\hline
\end{tabular}

Table 12. Comparison of database predicted chemicals with actual chemicals.

\begin{tabular}{|l|l|l|l|l|l|l|l|l|l|l|}
\hline \multicolumn{8}{|c|}{ A. Database } \\
\hline CAS & $\begin{array}{l}\text { Chemical } \\
\text { Name }\end{array}$ & State & $\begin{array}{l}\text { Exposure } \\
\text { Route }\end{array}$ & \multicolumn{1}{|l|}{ NFPA H/F/ } & \multicolumn{2}{l|}{ ITF40 (HSC/HPC/RR) } \\
\hline $7664-41-7$ & Ammonia & G & Inhalation & 3 & 1 & 0 & Negligible & Frequent & Moderate \\
\hline $67-56-1$ & Methanol & L & Oral & 3 & 3 & 0 & Critical & Likely & High \\
\hline \multicolumn{8}{|c|}{ B. Actual } \\
\hline CAS & $\begin{array}{l}\text { Chemical } \\
\text { Name }\end{array}$ & State & $\begin{array}{l}\text { Exposure } \\
\text { Route }\end{array}$ & NFPA H/F/I & ITF40 (HSC/HPC/RR) \\
\hline $1336-21-6$ & $\begin{array}{l}\text { Aqueous } \\
\text { Ammonia }\end{array}$ & L & Oral & 4 & & & Catastrophic & Occasional & High \\
\hline $64-17-5$ & Ethanol & L & Oral & 2 & 3 & 0 & Critical & Likely & High \\
\hline
\end{tabular}

Most probable chemicals

Table 13 summarizes the chemicals predicted to be most likely at the site. Two of these, sulfuric acid and sodium hydroxide, were found at the site. These were the second and third most likely chemicals, respectively. Ammonia was the eigth most likely chemical. As discussed in the section above, this chemical was similar, but not identical to, aqueous ammonia. The most likely chemical was n-hexane, which was actually not in the EPA report. However, this solvent chemical would likely serve the same role as ethanol, which was identified at the site.

Table 13. Ten most likely chemicals for Test 2.

\begin{tabular}{|l|l|l|}
\hline \%prob & CAS & Name \\
\hline 76.19 & 110543 & N-HEXANE \\
\hline 38.10 & 7664939 & SULFURIC ACID \\
\hline 26.19 & 1310732 & SODIUM HYDROXIDE (SOLUTION) \\
\hline 25.00 & 7664382 & PHOSPHORIC ACID \\
\hline 19.05 & 7647010 & HYDROCHLORIC ACID \\
\hline 14.29 & 7440020 & NICKEL \\
\hline 13.10 & 7782505 & CHLORINE \\
\hline 11.90 & 7664417 & AMMONIA \\
\hline 8.33 & 191242 & BENZO(G H I)PERYLENE \\
\hline 7.14 & 7439921 & LEAD \\
\hline
\end{tabular}




\section{Most significant chemical hazards}

The test team ranked the chemicals based on fire and flammability. Four chemicals in the original 26 chemical list had flammability rankings of 3 or higher (Table 14). The database was also used to rank the chemicals on toxicity (NFPA Health) (Table 15). Sulfuric acid (high health rating), ammonia ( 3 in health and 1 in flammability), and methanol ( 3 in health and 3 in flammability) were the most significant hazards identified by the test team. However, a good case could be made for several other chemicals.

Table 14. Ranking of Test 2 chemicals on fire/flammability: chemicals with NFPA-F ratings of 3 or higher.

\begin{tabular}{|l|l|l|l|}
\hline NFPA F & CAS & Chemical Name & \%Prob \\
\hline 4 & 115071 & PROPYLENE & 1.19 \\
\hline 3 & 110543 & N-HEXANE & 76.19 \\
\hline 3 & 67561 & METHANOL & 2.38 \\
\hline 3 & 67641 & ACETONE & 2.38 \\
\hline
\end{tabular}

Table 15. Ranking of Test 2 chemicals on toxicity/health: Chemicals with NFPA-H ratings of 3 or higher.

\begin{tabular}{|l|l|l|l|}
\hline NFPA H & CAS & Name & \%Prob \\
\hline 4 & 7664939 & SULFURIC ACID & 38.10 \\
\hline 4 & 7647010 & HYDROCHLORIC ACID & 19.05 \\
\hline 4 & 7782505 & CHLORINE & 13.10 \\
\hline 4 & 7439976 & MERCURY & 4.76 \\
\hline 4 & 7632000 & SODIUM NITRITE & 1.19 \\
\hline 3 & 7664417 & AMMONIA & 11.90 \\
\hline 3 & 67561 & METHANOL & 2.38 \\
\hline 3 & 91203 & NAPHTHALENE & 1.19 \\
\hline 3 & 92524 & BIPHENYL & 1.19 \\
\hline
\end{tabular}

Release modeling

A tank explosion was conducted using propylene, a gas with an NFPA flammability rating of "4." Explosion of a 4000-gal cylindrical tank was assumed, with a diameter of $10 \mathrm{ft}$ and a pressure of $75 \mathrm{psig}$. A relative humidity of 50\% and a single story, metal building were also assumed. 
ALOHA estimated a circular hazard pattern based on explosive energy (kwatts $/ \mathrm{m}^{2}$ ) (Figure 15a). The diameter of the circle of impact (lethal within 60 seconds) was $400 \mathrm{~m}$. In addition, the program estimated that a 30-m-diam fireball would be generated. Such an explosion would impact a significant portion of the harbor area and would potentially cut off traffic on the roads servicing the area (Figure 15b).

a. Size diagram of modeled explosion of a 4000-gal propylene tank.

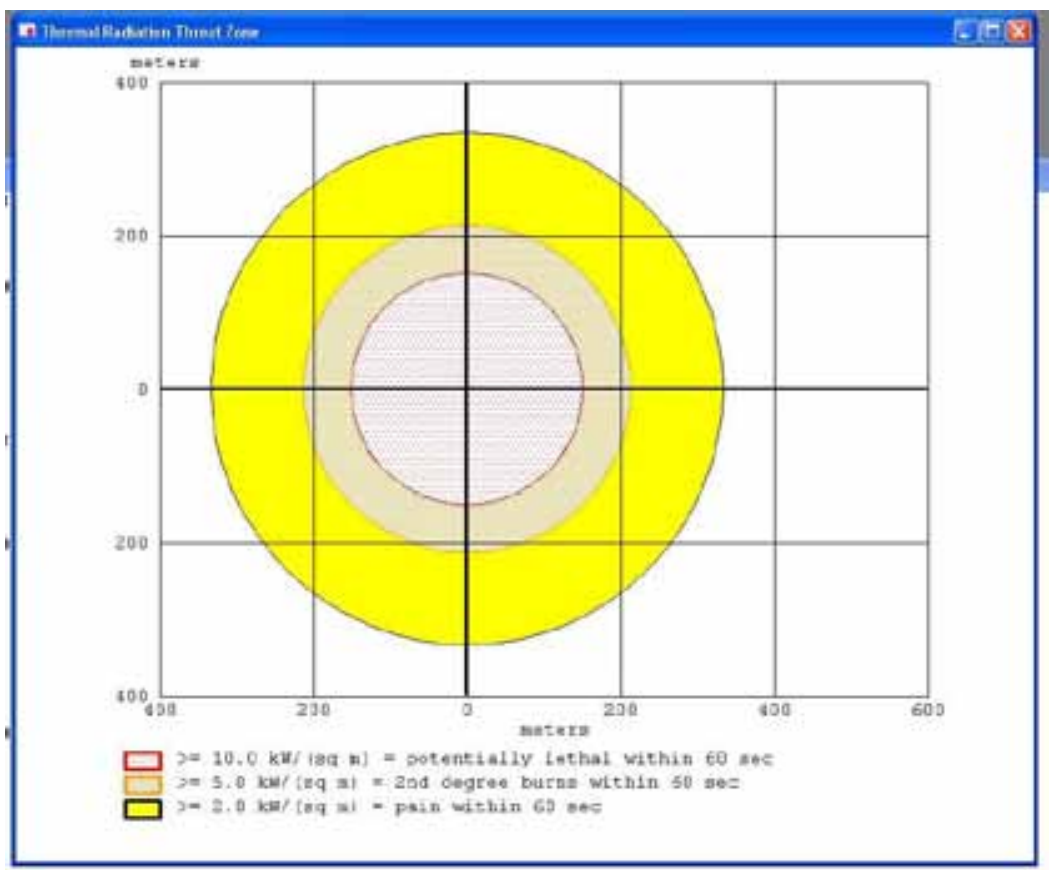

b. Explosion of a 4000-gal propylene tank for the facility in Test 2.

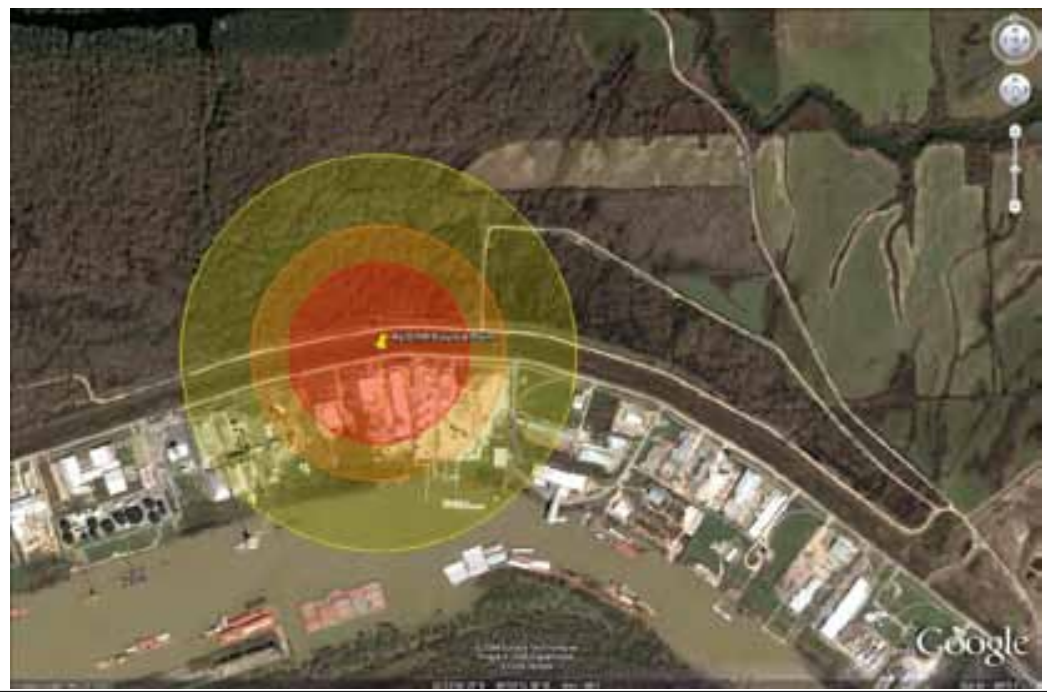

Figure 15. Release modeling of an explosion of a propylene tank for Test 2. 
SOPs from ITF-40 63 most significant list

Only one chemical was found with an SOP, hydrogen chloride, with this information derived from the SOP:

Hydrogen chloride - Its major concern is depression of $\mathrm{pH}$, creating acidic issues. It can cause severe burns. When diluted, it may not be a great hazard, but could mobilize metals. It can be neutralized, but care must be used because strong bases can explosively react with strong acids

\section{Test 3 Summary}

\section{Scenario}

The Test 3 scenario assumes multiple sites or industrial processes exist within a 1-square-mile area. At least one railroad car tank containing 7664-93-9 sulfuric acid was hit by a mortar shell. Surface soil modeling of 7664-93-9 is requested. Figure 16 is the submission sheet for the test.

\section{Results}

Response time

Due to sickness and differences in schedules, the team worked separately in increments of $1.5 \mathrm{hr}, 1.5 \mathrm{hr}$, and $1 \mathrm{hr}$, respectively. It probably would have taken approximately $2.5 \mathrm{hr}$ of labor if the team had worked together. This response time exceeded the goal of providing a respond within $2 \mathrm{hr}$.

\section{Facility identification}

The NAICS code: 336111 (automotive manufacturing) and 336112 (light truck and utility vehicle manufacturing) were correct. The test team's database only contained information for 336111, automotive manufacturing. However, it was reasonable to assume that the processes for automotive manufacturing and light truck manufacturing would be about the same. The 2008 Tier II report submitted by the Nissan North America, Inc., Canton Manufacturing Facility was used to verify the chemicals identified by the test team. 


\section{Test $\_$_ Scenario}

Date:10/26/09 Time Submitted:_0915 CDT_ Time Team Response Received:

Team Initial Start Time: Time Team Response Submitted:

\begin{tabular}{|c|c|c|}
\hline $\begin{array}{l}\text { Industrial Processes or } \\
\text { Process Name: } \\
\text { NAICS: } 336111 \text { and } 336112\end{array}$ & $\begin{array}{l}\text { TIC Release Mechanism: } \\
\text { The enemy has vacated this } \\
\text { area after a fierce aerial } \\
\text { assault. This assault resulted } \\
\text { in numerous fires at least } 8 \\
\text { hours ago. }\end{array}$ & $\begin{array}{l}\text { Atmospheric conditions: } \\
\text { Temperature: } 100^{\circ} \mathrm{F} \\
\text { Wind Speed: } 15 \\
\text { Wind Direction: SW } \\
\text { Precipitation: None }\end{array}$ \\
\hline $\begin{array}{l}\text { Suspected TICs: } \\
\text { At least one railroad car tank } \\
\text { containing } 7664-93-9 \text { sulfuric } \\
\text { acid was hit by a mortar shell. } \\
\text { Surface soil modeling of } \\
7664-93-9 \text { is requested. }\end{array}$ & Other Industrial Chemicals: & $\begin{array}{l}\text { Other Potential Hazards: } \\
\text { Flammable liquids are on } \\
\text { site. Drought conditions exist. }\end{array}$ \\
\hline $\begin{array}{l}\text { Site Designation: } \\
\text { Single Site: } \\
\text { Multiple Sites: } \\
\text { General Location*: } \underline{X}\end{array}$ & $\begin{array}{l}\text { Site A*: } \\
\text { Longitude: } 90^{\circ} 04,45.69^{\prime \prime} \mathrm{W} \\
\text { Latitude: } 32^{\circ} 35^{\prime} 17.42^{\prime \prime} \mathrm{N} \\
\text { Elevation: }\end{array}$ & $\begin{array}{l}\text { Site B: } \\
\text { Longitude: } \\
\text { Latitude: } \\
\text { Elevation: }\end{array}$ \\
\hline $\begin{array}{l}\text { Other Characteristics: } \\
\text { Assume multiple sites or } \\
\text { industrial processes exist } \\
\text { within a 1-square-mile area. } \\
\text { The area of concern includes } \\
\text { a half-mile distance in all four } \\
\text { directions from the } \\
\text { coordinates provided above. }\end{array}$ & & \\
\hline
\end{tabular}

Figure 16. Scenario description for Test 3.

Figure 17 is a location map. The skull and crossbones symbol marks the location of the facility located by the test team. However, the facility was apparently mislocated due to a discrepancy of the longitude and latitude provided. Perhaps the location of an office was provided, although technically the plant location should be reported in the Tier II report. The pink dot shows the actual location of the plant. 


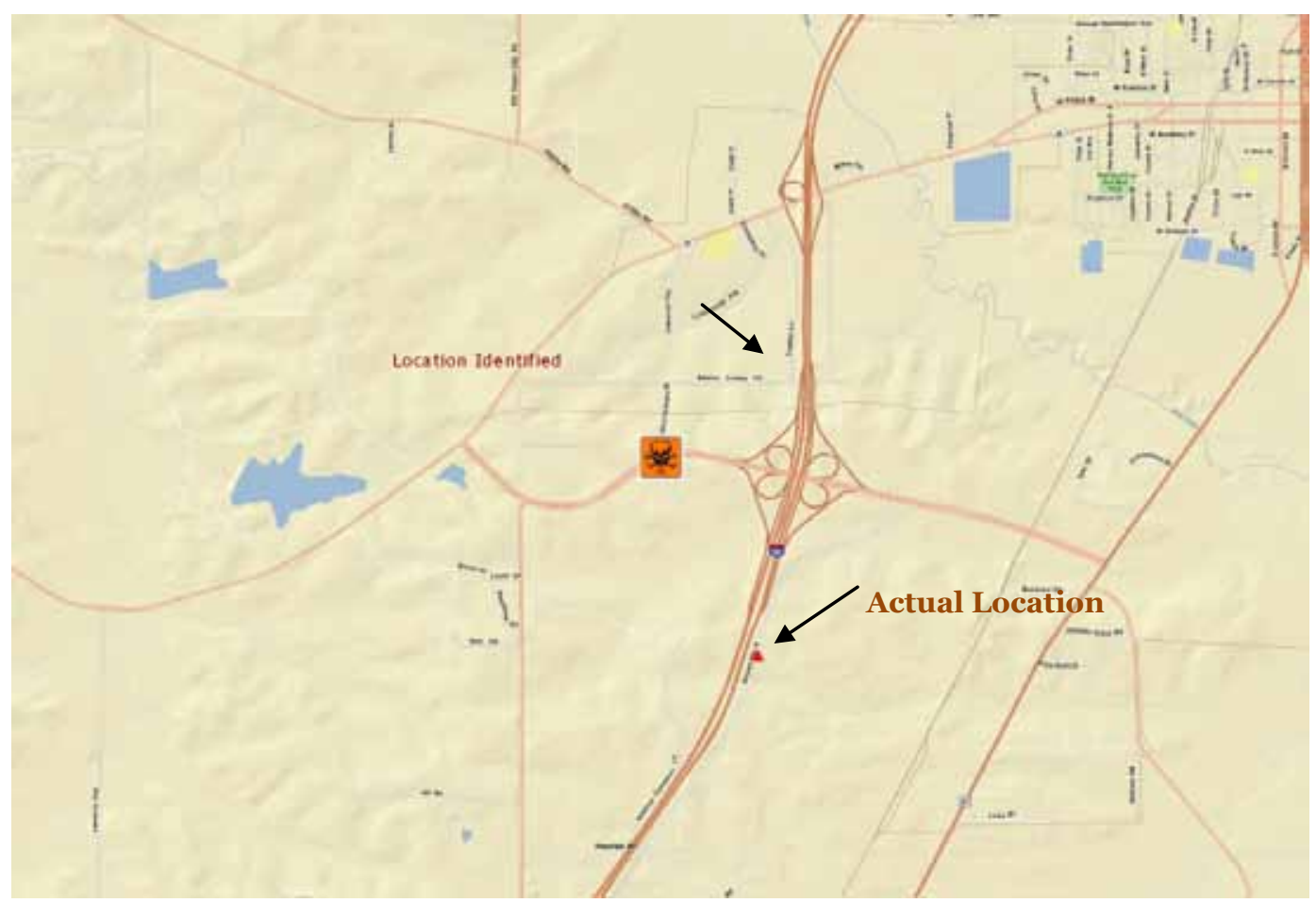

Figure 17. Location map for Test 3. Skull and crossbones symbol marks the identified location. The pink dot is the actual location.

\section{Chemical identification}

The test team identified 91 potential TICs (Table 16). Fifty-three chemicals were listed on the Tier II report from Nissan. Of the 53, 30 were actually in the program database (Table 17). The database correctly identified 15 of the 30 remaining chemicals (Table 18).

\section{Ten most likely chemicals}

The test team identified the 10 most probable chemicals (Table 19), six of which were included in the Nissan Tier II report.

\section{Most significant chemical hazards}

Tables 20 and 21 show the highest-rated chemical hazards for fire/flammability and for toxicity/health for the chemicals identified in Table 19. The test team chose the top three risks to be sulfuric acid (4 health risk and identified by the scenario as a high probability of release), benzene ( 4 health risk, and 3 flammability), and vinylidene chloride ( 4 fire risk, 3 health) (Table 22). Strong cases could be made for other chemicals as well. 
Table 16. Estimated chemical list for Test 3.

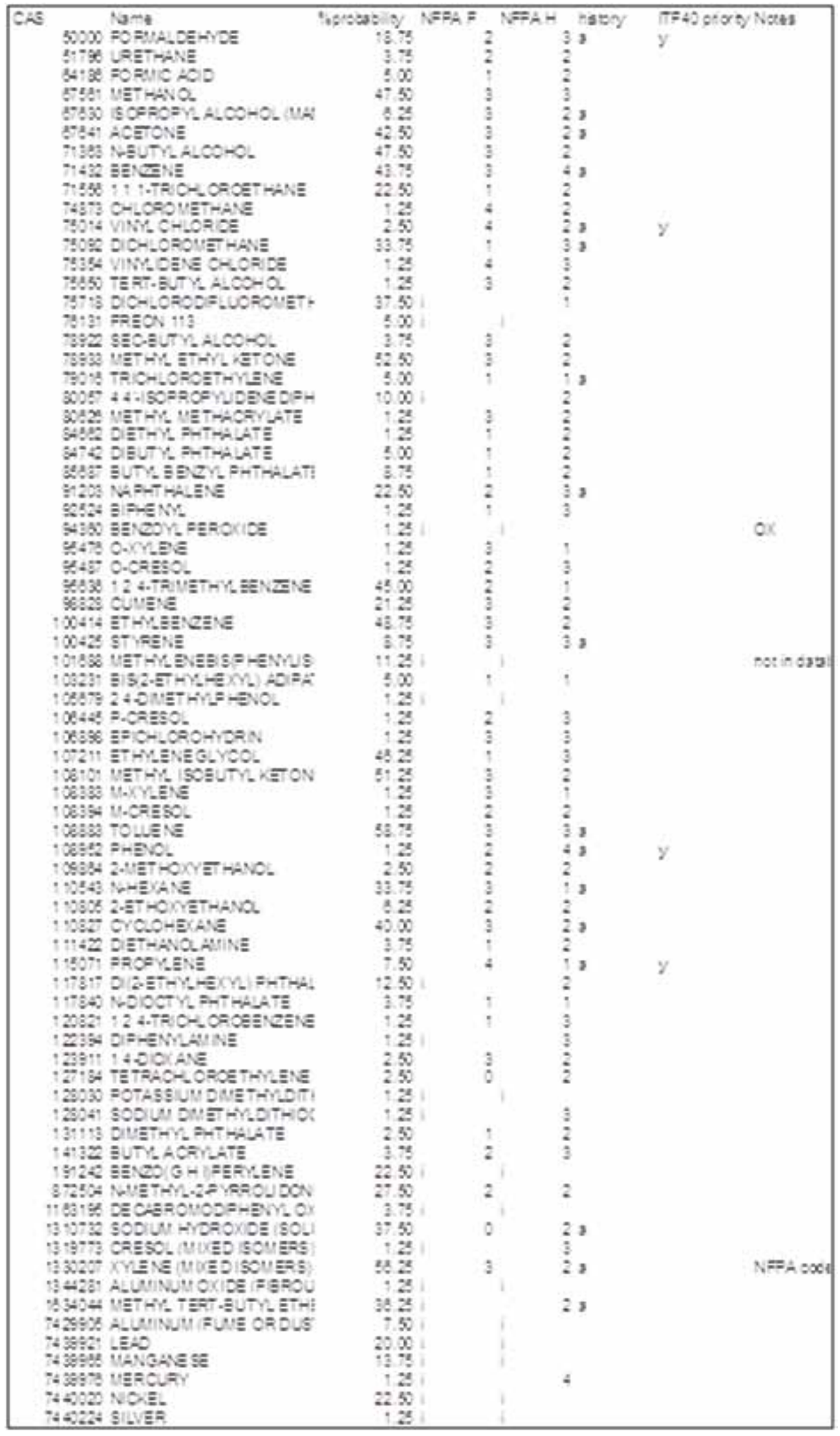


Table 17. Chemicals listed on Nissan Tier II report and the TIC-Master database.

\begin{tabular}{|c|c|c|c|c|}
\hline CAS_Text & Chemical_Name & State & \begin{tabular}{|l|} 
Inventory \\
(Max Daily lb)
\end{tabular} & \begin{tabular}{|l|} 
Inventory \\
(Ave Daily lb)
\end{tabular} \\
\hline 7664-93-9 & Sulfuric acid & L & $10,000-99,999$ & $10,000-99,999$ \\
\hline $107-21-1$ & Ethane-1,2-diol & L & $100,000-999,999$ & $100,000-999,999$ \\
\hline $75-65-0$ & 2-methylpropan-2-ol & L & $10,000-99,999$ & $10,000-99,999$ \\
\hline $1310-73-2$ & Sodium hydroxide & $S$ & $10,000-99,999$ & $10,000-99,999$ \\
\hline $8008-20-6$ & Kerosene & $\mathrm{L}$ & $10,000-99,999$ & $10,000-99,999$ \\
\hline $763-69-9$ & Ethyl 3-ethoxypropionate & $\mathrm{L}$ & $10,000-99,999$ & $10,000-99,999$ \\
\hline $7705-08-0$ & Iron trichloride & $L$ & $100,000-999,999$ & $100,000-999,999$ \\
\hline $67-63-0$ & Propan-2-ol & L & $10,000-99,999$ & $10,000-99,999$ \\
\hline $78-93-3$ & Butanone & $\mathrm{L}$ & $1,000-9,999$ & $1,000-9,999$ \\
\hline $123-86-4$ & n-butyl acetate & L & $100,000-999,999$ & $100,000-999,999$ \\
\hline $811-97-2$ & 1,1,1,2-Tetrafluoroethane & $G$ & $10,000-99,999$ & $10,000-99,999$ \\
\hline $5131-66-8$ & 2-Propanol, 1-butoxy-(PGnBE) & L & $100,000-999,999$ & $100,000-999,999$ \\
\hline $67-56-1$ & Methanol & $\mathrm{L}$ & $10,000-99,999$ & $10,000-99,999$ \\
\hline $1305-78-8$ & Calcium oxide & $S$ & $100,000-999,999$ & $10,000-99,999$ \\
\hline $497-19-8$ & Sodium carbonate & S & $10,000-99,999$ & $1,000-9,999$ \\
\hline $112-80-1$ & Oleic acid & L & $10,000-99,999$ & $10,000-99,999$ \\
\hline $1310-58-3$ & Potassium hydroxide & $S$ & $10,000-99,999$ & $10,000-99,999$ \\
\hline 7697-37-2 & Nitric acid & L & $10,000-99,999$ & $10,000-99,999$ \\
\hline $95-63-6$ & 1,2,4-trimethylbenzene & L & & \\
\hline $112-25-4$ & 2-hexyloxyethanol & L & $10,000-99,999$ & $10,000-99,999$ \\
\hline $7632-00-0$ & Sodium nitrite & $S$ & $10,000-99,999$ & $10,000-99,999$ \\
\hline $7631-99-4$ & Sodium nitrate & $S$ & $10,000-99,999$ & $10,000-99,999$ \\
\hline 108-10-1 & 4-methylpentan-2-one & $L$ & $10,000-99,999$ & $10,000-99,999$ \\
\hline $124-38-9$ & Carbon dioxide & G & $10,000-99,999$ & $10,000-99,999$ \\
\hline $7727-37-9$ & Nitrogen, compressed & G & $10,000-99,999$ & $10,000-99,999$ \\
\hline $6846-50-0$ & $\begin{array}{l}\text { 1-isopropyl-2,2-dimethyltrimethylene } \\
\text { diisobutyrate }\end{array}$ & & & \\
\hline 67-64-1 & Acetone & L & 1,000-9,999 & 1,000-9,999 \\
\hline 7647-01-0 & Hydrogen chloride & $G$ & $100,000-999,999$ & 10,000-99,999 \\
\hline $872-50-4$ & 1-methyl-2-pyrrolidone & $L$ & $10,000-99,999$ & $10,000-99,999$ \\
\hline $306-83-2$ & Ethane, 2,2-dichloro-1,1,1-trifluoro- & L & $10,000-99,999$ & $10,000-99,999$ \\
\hline
\end{tabular}

blue font: Chemicals on the ITF-40 Top 63 list

State Symbols: $\mathrm{S}=$ Solid, $\mathrm{L}=$ Liquid, $\mathrm{G}=$ Gas. 
Table 18. Fifteen chemicals identified by the test team appear in the Tier II report and TIC-Master database.

\begin{tabular}{|l|l|}
\hline CAS_Text & Chemical Identified by the Test Team \\
\hline $67-56-1$ & Methanol \\
\hline $67-63-0$ & Propan-2-ol \\
\hline $67-64-1$ & Acetone \\
\hline $71-36-3$ & $\begin{array}{l}\text { Butan-1-ol } \\
\text { (n-butyl alcohol) }\end{array}$ \\
\hline $75-65-0$ & 2-methylpropan-2-ol \\
\hline $78-93-3$ & $\begin{array}{l}\text { Butanone } \\
\text { (methyl ethyl ketone) }\end{array}$ \\
\hline $95-63-6$ & $1,2,4-$ trimethylbenzene \\
\hline $107-21-1$ & $\begin{array}{l}\text { Ethane-1,2-diol } \\
\text { (ethylene glycol) }\end{array}$ \\
\hline $108-10-1$ & $\begin{array}{l}\text { 4-methylpentan-2-one } \\
\text { (methyl isobutyl ketone) }\end{array}$ \\
\hline $872-50-4$ & 1-methyl-2-pyrrolidone \\
\hline $1310-73-2$ & Sodium hydroxide \\
\hline $7632-00-0$ & Sodium nitrite \\
\hline $7647-01-0$ & Hydrogen chloride \\
\hline $7664-93-9$ & Sulfuric acid \\
\hline $7697-37-2$ & Nitric acid \\
\hline
\end{tabular}

Table 19. Most probable chemicals for Test 3. Starred Items were included in the actual Tier II report.

\begin{tabular}{|l|l|l|}
\hline \%prob & CAS & Name \\
\hline 58.75 & 108883 & TOLUENE \\
\hline 56.25 & 1330207 & XYLENE (MIXED ISOMERS) \\
\hline 52.50 & 78933 & METHYL ETHYL KETONE \\
\hline 51.25 & 108101 & METHYL ISOBUTYL KETONE \\
\hline 48.75 & 100414 & ETHYLBENZENE \\
\hline 47.50 & 67561 & METHANOL \\
\hline 47.50 & 71363 & N-BUTYL ALCOHOL \\
\hline 47.50 & 7664939 & SULFURIC ACID \\
\hline 46.25 & 107211 & ETHYLENE GLYCOL \\
\hline 46.25 & 7664382 & PHOSPHORIC ACID \\
\hline
\end{tabular}

Note: 37 chemicals with $10 \%$ or greater probability 
Table 20. Chemical hazard list for Test 3 based on fire/flammability (NFPA-F). All chemicals with rankings of 3 or greater.

\begin{tabular}{|c|c|c|c|c|}
\hline NFPA F & CAS \# & CHEMICAL NAME & \%Prob & NFPA H \\
\hline 4 & 115071 & PROPYLENE & 7.50 & 1 \\
\hline 4 & 75014 & VINYL CHLORIDE & 2.50 & 2 \\
\hline 4 & 74873 & CHLOROMETHANE & 1.25 & 2 \\
\hline 4 & 75354 & VINYLIDENE CHLORIDE & 1.25 & 3 \\
\hline 3 & 108883 & TOLUENE & 58.75 & 3 \\
\hline 3 & 1330207 & XYLENE (MIXED ISOMERS) & 56.25 & 2 \\
\hline 3 & 78933 & METHYL ETHYL KETONE & 52.50 & 2 \\
\hline 3 & 108101 & METHYL ISOBUTYL KETONE & 51.25 & 2 \\
\hline 3 & 100414 & ETHYLBENZENE & 48.75 & 2 \\
\hline 3 & 67561 & METHANOL & 47.50 & 3 \\
\hline 3 & 71363 & N-BUTYL ALCOHOL & 47.50 & 2 \\
\hline 3 & 71432 & BENZENE & 43.75 & 4 \\
\hline 3 & 67641 & ACETONE & 42.50 & 2 \\
\hline 3 & 110827 & CYCLOHEXANE & 40.00 & 2 \\
\hline 3 & 110543 & N-HEXANE & 33.75 & 1 \\
\hline 3 & 98828 & CUMENE & 21.25 & 2 \\
\hline 3 & 100425 & STYRENE & 8.75 & 3 \\
\hline 3 & 67630 & ISOPROPYL ALCOHOL & 6.25 & 2 \\
\hline 3 & 78922 & SEC-BUTYL ALCOHOL & 3.75 & 2 \\
\hline 3 & 123911 & 1 4-DIOXANE & 2.50 & 2 \\
\hline 3 & 75650 & TERT-BUTYL ALCOHOL & 1.25 & 2 \\
\hline 3 & 80626 & METHYL METHACRYLATE & 1.25 & 2 \\
\hline 3 & 95476 & O-XYLENE & 1.25 & 1 \\
\hline 3 & 106898 & EPICHLOROHYDRIN & 1.25 & 3 \\
\hline 3 & 108383 & M-XYLENE & 1.25 & 1 \\
\hline
\end{tabular}


Table 21. Chemical hazard list for Test 3 based on toxicity/health (NFPA-H). All chemicals with rankings of 3 or greater.

\begin{tabular}{|c|c|c|c|c|}
\hline NFPA H & CAS & Chemical Name & $\%$ prob & NFPA F \\
\hline 4 & 7664939 & SULFURIC ACID & 47.50 & 0 \\
\hline 4 & 71432 & BENZENE & 43.75 & 3 \\
\hline 4 & 7632000 & SODIUM NITRITE & 43.75 & i \\
\hline 4 & 7697372 & NITRIC ACID & 41.25 & 0 \\
\hline 4 & 7647010 & HYDROCHLORIC ACID & 35.00 & 0 \\
\hline 4 & 7782505 & CHLORINE & 6.25 & 0 \\
\hline 4 & 7723140 & $\begin{array}{l}\text { PHOSPHORUS (YELLOW OR } \\
\text { WHITE) }\end{array}$ & 5.00 & i \\
\hline 4 & 108952 & PHENOL & 1.25 & 2 \\
\hline 4 & 7439976 & MERCURY & 1.25 & i \\
\hline 4 & 7440439 & CADMIUM & 1.25 & i \\
\hline 4 & 7664393 & HYDROGEN FLUORIDE & 1.25 & 0 \\
\hline 3 & 108883 & TOLUENE & 58.75 & 3 \\
\hline 3 & 67561 & METHANOL & 47.50 & 3 \\
\hline 3 & 107211 & ETHYLENE GLYCOL & 46.25 & 1 \\
\hline 3 & 75092 & DICHLOROMETHANE & 33.75 & 1 \\
\hline 3 & 91203 & NAPHTHALENE & 22.50 & 2 \\
\hline 3 & 50000 & FORMALDEHYDE & 18.75 & 2 \\
\hline 3 & 100425 & STYRENE & 8.75 & 3 \\
\hline 3 & 7664417 & AMMONIA & 5.00 & 1 \\
\hline 3 & 141322 & BUTYL ACRYLATE & 3.75 & 2 \\
\hline 3 & 75354 & VINYLIDENE CHLORIDE & 1.25 & 4 \\
\hline 3 & 92524 & BIPHENYL & 1.25 & 1 \\
\hline 3 & 95487 & O-CRESOL & 1.25 & 2 \\
\hline 3 & 106445 & P-CRESOL & 1.25 & 2 \\
\hline 3 & 106898 & EPICHLOROHYDRIN & 1.25 & 3 \\
\hline 3 & 120821 & 12 4-TRICHLOROBENZENE & 1.25 & 1 \\
\hline 3 & 122394 & DIPHENYLAMINE & 1.25 & i \\
\hline 3 & 128041 & $\begin{array}{l}\text { SODIUM } \\
\text { DIMETHYLDITHIOCARBAMATE }\end{array}$ & 1.25 & i \\
\hline 3 & 1319773 & CRESOL (MIXED ISOMERS) & 1.25 & i \\
\hline
\end{tabular}


Table 22. Three most significant chemical hazards selected by the test team.

\begin{tabular}{|l|l|l|}
\hline Chemicals & Health & Fire \\
\hline Sulfuric Acid & 4 & 0 \\
\hline Benzene & 4 & 3 \\
\hline Vinylidene Chloride & 4 & 3 \\
\hline
\end{tabular}

\section{Release modeling}

The scenario stated "At least one railroad car tank containing 7664-93-9 sulfuric acid was hit by a mortar shell. Surface soil modeling of 7664-93-9 is requested." In this case, the ALOHA model's limitations prevented modeling of the scenario presented. First, ALOHA does not include surface release modeling. However, it does allow for modeling of air contamination from a surface release; therefore efforts were focused on modeling the atmospheric effects. Secondly, ALOHA does not include sulfuric acid as one of its chemicals. The available acids were reviewed, including nitric acid, hydrochloric acid, hydrofluoric acid, and sulfurous acid. Sulfurous acid was investigated and found to be different than sulfuric acid in chemical behavior. Sulfurous acid also required inputs that were not available. HF acid, which is generally considered the worst of the strong acids, modeled as a worst-case scenario.

The model focused on the air effects of a release of 100 gal of HF, substantially less than a railcar. This was an oversight by the response team. The modeling assumed that the release was from $2 \times 55$ gallon drums spilled on the ground. The release included the weather conditions given in the start conditions. As seen, the surface spill release from a circular tank creates a plume-shaped area of influence that is greatly impacted by the wind (Figure 18a).

Figure $18 \mathrm{~b}$ shows the modeled plume. The plume was rather narrow in diameter, less than $0.1 \mathrm{~km}$. The 44-ppm portion of the plume was about $0.1 \mathrm{~km}$ long. However, the 1-ppm portion of the plume, which is great enough to affect the eyes and respiratory system, extended for a length of $1 \mathrm{~km}$. 


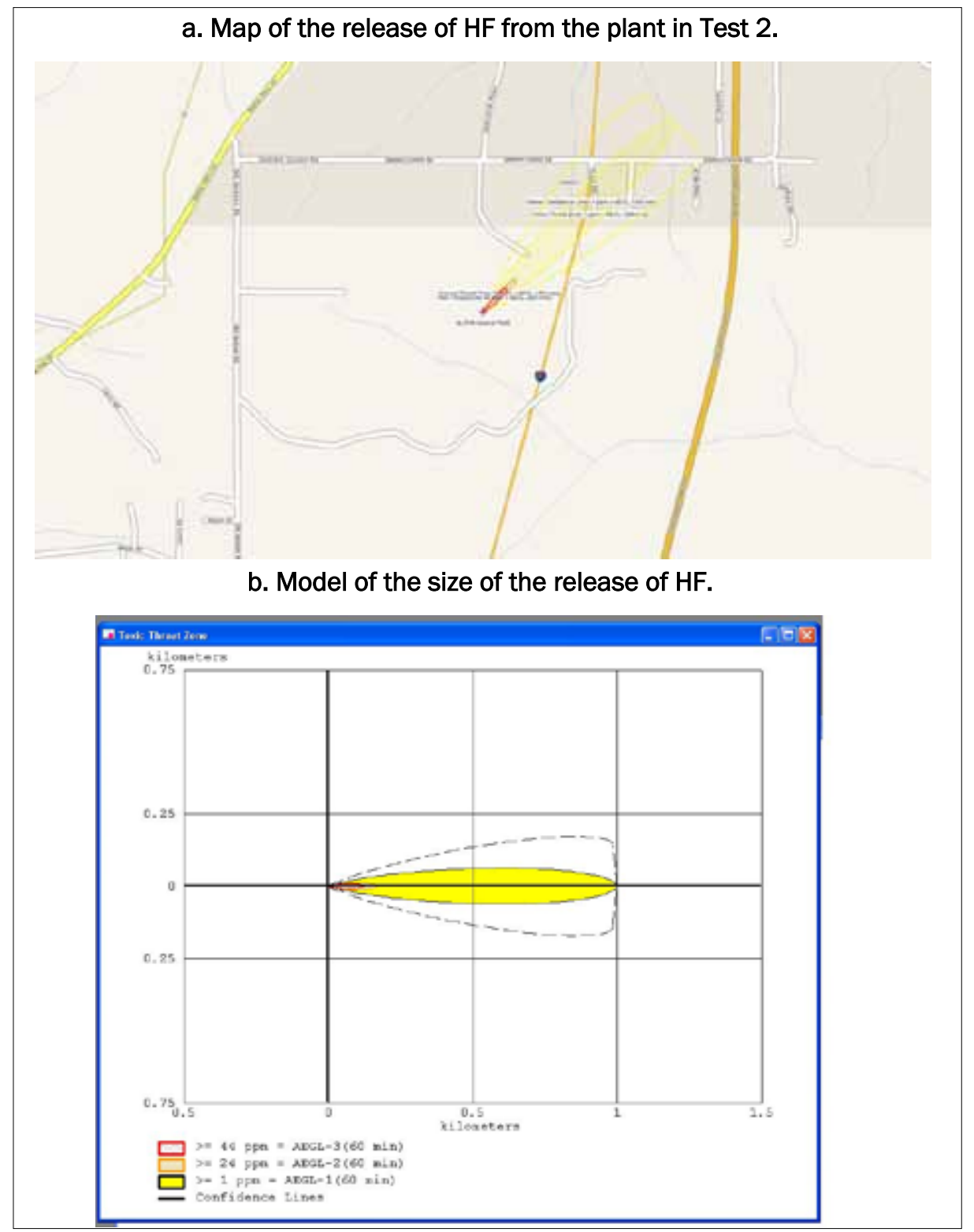

Figure 18. Release modeling supporting Test 3.

\section{Test 4 Summary}

\section{Scenario}

Coordinated suicide bombers detonated trucks at two facilities. Massive fires ensued. A partial chemical list is available for each facility. The commander needs to address community concerns about hydrogen sulfide (CAS \# 7783-06-4) in a nearby waterway. Figure 19 is the test scenario submission sheet. 


\section{Test_4__Scenario}

Date:11/2/09 Time Submitted:_0945 CST__ Time Team Response Received:

Team Initial Start Time:___ Time Team Response Submitted:

We estimate our working time on this to be on the order of 2.75 hours.

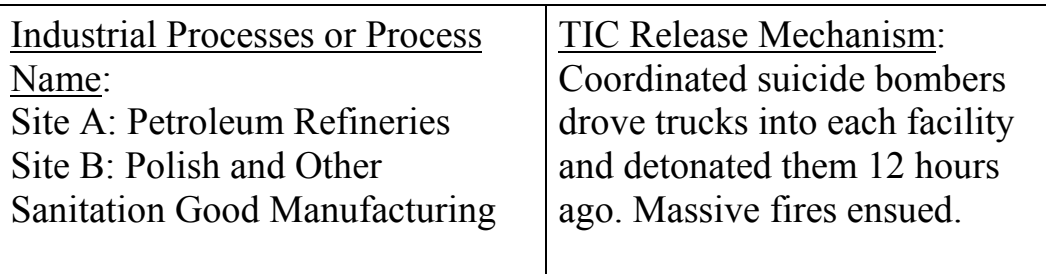

TICs:

Site A: Chemicals include 007647-01-0 Muriatic acid, 001310-73-2

Caustic, 8002-26-4 Distilled Toll Oil, and 7783-06-4 Hydrogen sulfide.

Note: This is only a partial chemical list.

Site B: Chemicals include 7664-93-9 Battery acid, 68608-26-4

Hostapur, 7439-92-1 Lead, 57-55-6 Propylene glycol, 1310-73-2

Sodium hydroxide (Caustic), and 67-63-0 Isopropyl alcohol-

anhydrous.

Atmospheric conditions:

Temperature: $24 \mathrm{deg} \mathrm{F}$

Wind Speed: $10 \mathrm{mph}$

Wind Direction: East

Precipitation: 0.25 inch per hour

\section{Other Potential Hazards:}

Site A: Other potential hazards on site include 64741-44-2

diesel, 8008-20-6 Kerosene, and unleaded gasoline.

Site B: Other potential hazards on site include 65-85-0 Benzoic acid, 8002-09-3 Terpene alcohol/ hydrocarbons, and original pine sol.

Note: This is only a partial chemical list.

\begin{tabular}{|c|c|c|}
\hline $\begin{array}{l}\text { Site Designation: } \\
\text { Single Site: } \\
\text { Multiple Sites: } \quad \underline{X} \\
\text { General Location*: }\end{array}$ & $\begin{array}{l}\text { Site A*: } \\
\text { Longitude: } 90^{\circ} 54^{\prime} 21.20^{\prime \prime} \mathrm{W} \\
\text { Latitude: } 32^{\circ} 2319.63 ” \mathrm{~N} \\
\text { Elevation: }\end{array}$ & $\begin{array}{l}\text { Site B: } \\
\text { Longitude: } 90^{\circ} 09^{\prime} 26.06^{\prime \prime} \mathrm{W} \\
\text { Latitude: } 32^{\circ} 17^{\prime} 03.09^{\prime \prime} \mathrm{N} \\
\text { Elevation: }\end{array}$ \\
\hline
\end{tabular}

Other Characteristics:

Site A: Can you address community concerns about 7783-

06-4 Hydrogen sulfide

concentrations in a nearby

waterway?

* See the Other Characteristics section. For example, the U.S. Army may wish to occupy a 2-squaremile area centered at the location provided by Site A*.

Figure 19. Scenario description for Test 4. 


\section{Results}

Response time

The estimated working time was $2.75 \mathrm{hr}$. This response time exceeded the goal of $2 \mathrm{hr}$.

\section{Facility identification}

Site A referred to Ergon Refining, Inc., and was identified as a chemical refining industry. Site B referred to the Clorox Products Manufacturing Co. NAICS: 325612 and was identified by the TIC-Master database as cleaning and sanitation products manufacturing. Figure 20 is a site location map. The red date refers to Site A. Site B was located by the skull and crossbones symbol.

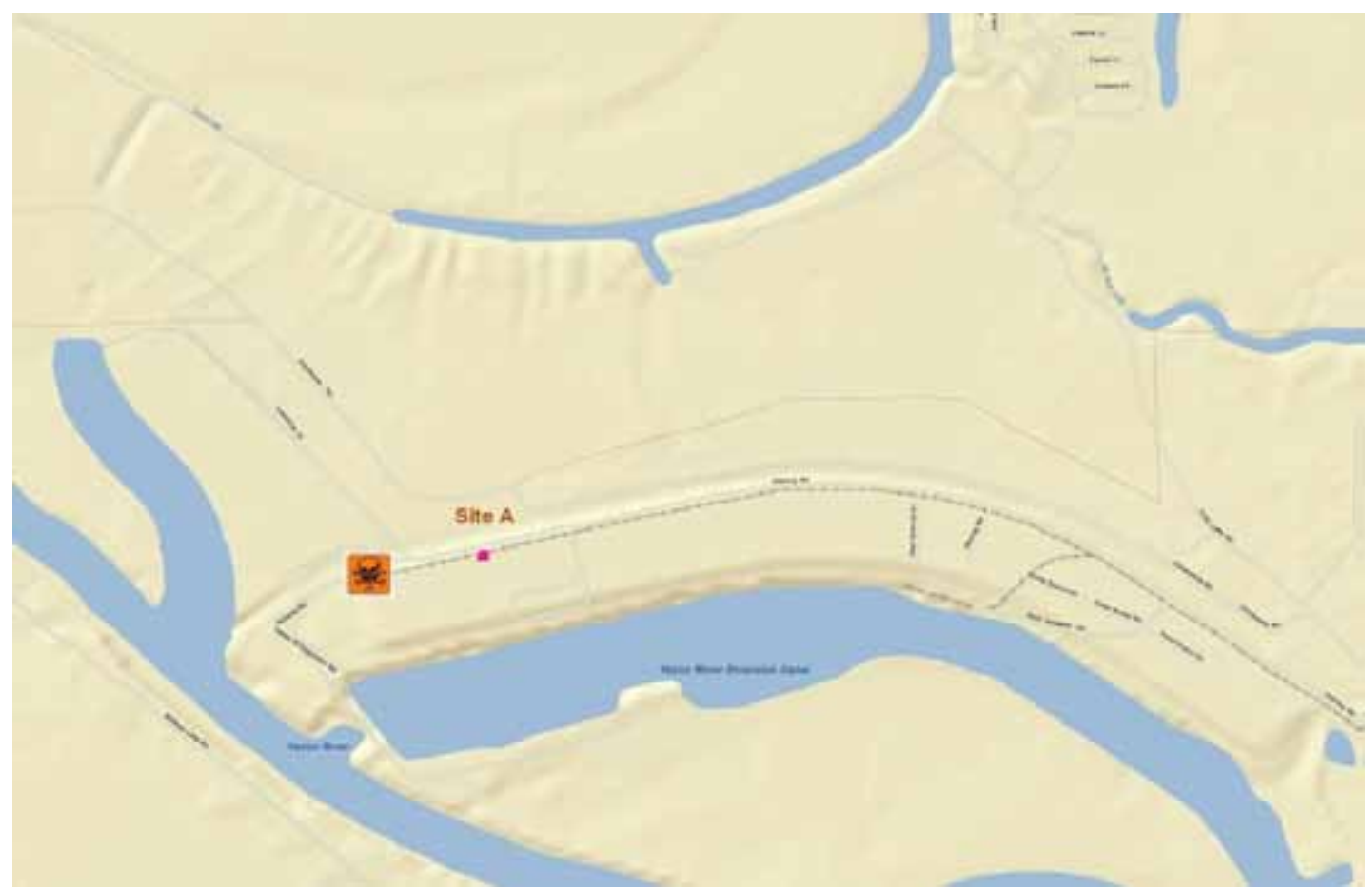

Figure 20. Location map for Test 4. The pink dot is Site A. Skull and crossbones was Site B.

\section{Chemical identification}

A total of 160 possible chemicals were identified for the chemical refining operation (Table 23). Forty-four chemicals were actually identified in the EPA Tier II report for Ergon Refining, Inc. Of these, 11 were TICs found in the TIC-Master database. Four of these 11 were identified by the TIC-Master database. Tables 24 and 25 show the TICs identified and not identified, respectively. 
Table 23. Full listing of chemicals predicted for chemical refining, with the first $\mathbf{3 0}$ ranked.

\begin{tabular}{|c|c|c|c|c|c|}
\hline CHEMICAL & \%Prob & Ranking & CHEMICAL & \%Prob & Ranking \\
\hline TOLUENE & 79.65 & 1 & 12-DICHLOROETHANE & 20.00 & \\
\hline BENZENE & 78.95 & 2 & CHROMIUM & 19.30 & \\
\hline XYLENE (MIXED ISOMERS) & 76.84 & 3 & BIPHENYL & 16.49 & \\
\hline ETHYLBENZENE & 73.33 & 4 & METHYL ETHYL KETONE & 16.14 & \\
\hline CYCLOHEXANE & 69.82 & 5 & ANTHRACENE & 15.09 & \\
\hline 12 4-TRIMETHYLBENZENE & 64.91 & 6 & O-XYLENE & 14.74 & \\
\hline NAPHTHALENE & 62.11 & 7 & PHENANTHRENE & 14.39 & \\
\hline SULFURIC ACID & 61.75 & 8 & 12-DIBROMOETHANE & 14.39 & \\
\hline N-HEXANE & 59.30 & 9 & HYDROGEN CYANIDE & 13.33 & \\
\hline SODIUM HYDROXIDE (SOLUTION) & 58.25 & 10 & P-XYLENE & 13.33 & \\
\hline AMMONIA & 55.44 & 11 & M-XYLENE & 13.33 & \\
\hline CHLORINE & 53.68 & 12 & CARBON TETRACHLORIDE & 12.98 & \\
\hline PROPYLENE & 50.18 & 13 & 2-METHOXYETHANOL & 12.28 & \\
\hline BENZO(G H I)PERYLENE & 48.77 & 14 & VANADIUM (FUME OR DUST) & 11.93 & \\
\hline METHYL TERT-BUTYL ETHER & 48.42 & 15 & COBALT & 11.23 & \\
\hline ALUMINUM OXIDE (FIBROUS FORMS) & 47.37 & 16 & MANGANESE & 10.88 & \\
\hline ETHYLENE & 47.02 & 17 & TERT-BUTYL ALCOHOL & 9.47 & \\
\hline CUMENE & 47.02 & 18 & FORMALDEHYDE & 8.07 & \\
\hline METHANOL & 44.91 & 19 & N-BUTYL ALCOHOL & 8.07 & \\
\hline MOLYBDENUM TRIOXIDE & 42.81 & 20 & COPPER & 8.07 & \\
\hline 13-BUTADIENE & 40.35 & 21 & ASBESTOS (FRIABLE) & 7.72 & \\
\hline PHENOL & 39.30 & 22 & BARIUM & 7.72 & \\
\hline HYDROCHLORIC ACID & 34.04 & 23 & ZINC (FUME OR DUST) & 7.37 & \\
\hline DIETHANOLAMINE & 33.33 & 24 & ANTIMONY & 6.67 & \\
\hline PHOSPHORIC ACID & 31.93 & 25 & 24-DIMETHYLPHENOL & 6.32 & \\
\hline TETRACHLOROETHYLENE & 31.23 & 26 & ACETONE & 5.61 & \\
\hline LEAD & 30.88 & 27 & TRICHLOROETHYLENE & 4.91 & \\
\hline CARBONYL SULFIDE & 28.07 & 28 & METHYL ISOBUTYL KETONE & 4.91 & \\
\hline CARBON DISULFIDE & 26.67 & 29 & CHLORODIFLUOROMETHANE & 4.56 & \\
\hline ETHYLENE GLYCOL & 26.32 & 30 & DICHLORODIFLUOROMETHANE & 4.21 & \\
\hline 111-TRICHLOROETHANE & 24.91 & & POLYCHLORINATED BIPHENYLS & 4.21 & \\
\hline NICKEL & 23.16 & & ARSENIC & 4.21 & \\
\hline CRESOL (MIXED ISOMERS) & 22.81 & & DICYCLOPENTADIENE & 3.86 & \\
\hline HYDROGEN FLUORIDE & 22.81 & & N-METHYL-2-PYRROLIDONE & 3.86 & \\
\hline MERCURY & 20.70 & & CADMIUM & 3.86 & \\
\hline STYRENE & 20.35 & & SELENIUM & 3.86 & \\
\hline
\end{tabular}




\begin{tabular}{|c|c|c|c|c|c|}
\hline CHEMICAL & \%Prob & Ranking & CHEMICAL & \%Prob & Ranking \\
\hline SODIUM NITRITE & 3.51 & & ACRYLIC ACID & 0.70 & \\
\hline DICHLOROMETHANE & 3.16 & & METHYL METHACRYLATE & 0.70 & \\
\hline SEC-BUTYL ALCOHOL & 2.81 & & QUINOLINE & 0.70 & \\
\hline BERYLLIUM & 2.81 & & 12 3-TRICHLOROPROPANE & 0.70 & \\
\hline HYDRAZINE & 2.46 & & ACETOPHENONE & 0.70 & \\
\hline AMMONIUM SULFATE (SOLUTION) & 2.46 & & P-CRESOL & 0.70 & \\
\hline ACETALDEHYDE & 2.11 & & P-PHENYLENEDIAMINE & 0.70 & \\
\hline FREON 113 & 2.11 & & 12-BUTYLENE OXIDE & 0.70 & \\
\hline CHLORINE DIOXIDE & 2.11 & & EPICHLOROHYDRIN & 0.70 & \\
\hline ISOPROPYL ALCOHOL & 1.75 & & ALLYL ALCOHOL & 0.70 & \\
\hline TRICHLOROFLUOROMETHANE & 1.75 & & PYRIDINE & 0.70 & \\
\hline 1 2-DICHLOROPROPANE & 1.75 & & HEXACHLOROBENZENE & 0.70 & \\
\hline BUTYL BENZYL PHTHALATE & 1.75 & & HYDROQUINONE & 0.70 & \\
\hline PHOSPHORUS (YELLOW OR WHITE) & 1.75 & & BUTYRALDEHYDE & 0.70 & \\
\hline ANILINE & 1.40 & & DIMETHYLAMINE & 0.70 & \\
\hline CHLOROFORM & 1.40 & & 12-DICHLOROETHYLENE & 0.70 & \\
\hline ETHYLENE OXIDE & 1.40 & & AMMONIUM NITRATE (SOLUTION) & 0.70 & \\
\hline CHLOROBENZENE & 1.40 & & THALLIUM & 0.70 & \\
\hline 12 4-TRICHLOROBENZENE & 1.40 & & VINYL CHLORIDE & 0.35 & \\
\hline ALUMINUM (FUME OR DUST) & 1.40 & & BROMOFORM & 0.35 & \\
\hline SILVER & 1.40 & & ETHYLIDENE DICHLORIDE & 0.35 & \\
\hline NITRIC ACID & 1.40 & & BROMOTRIFLUOROMETHANE & 0.35 & \\
\hline N N-DIMETHYLFORMAMIDE & 1.05 & & DIMETHYL SULFATE & 0.35 & \\
\hline CHLOROMETHANE & 1.05 & & 2 3-DICHLOROPROPENE & 0.35 & \\
\hline ACETONITRILE & 1.05 & & PERACETIC ACID & 0.35 & \\
\hline PROPYLENE OXIDE & 1.05 & & 112 2-TETRACHLOROETHANE & 0.35 & \\
\hline 112 -TRICHLOROETHANE & 1.05 & & 4 4'-ISOPROPYLIDENEDIPHENOL & 0.35 & \\
\hline O-CRESOL & 1.05 & & CUMENE HYDROPEROXIDE & 0.35 & \\
\hline ACROLEIN & 1.05 & & PHTHALIC ANHYDRIDE & 0.35 & \\
\hline VINYL ACETATE & 1.05 & & 26-XYLIDINE & 0.35 & \\
\hline M-CRESOL & 1.05 & & TOLUENE-2 6-DIISOCYANATE & 0.35 & \\
\hline DIBENZOFURAN & 1.05 & & BENZIDINE & 0.35 & \\
\hline 13 -DICHLOROPROPYLENE & 1.05 & & 12-DICHLOROBENZENE & 0.35 & \\
\hline FLUORINE & 1.05 & & 24 5-TRICHLOROPHENOL & 0.35 & \\
\hline FORMIC ACID & 0.70 & & NITROBENZENE & 0.35 & \\
\hline BROMOMETHANE & 0.70 & & METHYLENEBIS(PHENYLISOCYANATE) & 0.35 & \\
\hline CHLOROTRIFLUOROMETHANE & 0.70 & & ACRYLONITRILE & 0.35 & \\
\hline
\end{tabular}




\begin{tabular}{|l|l|l||l|l|l|}
\hline CHEMICAL & \%Prob & Ranking & CHEMICAL & \%Prob & Ranking \\
\hline MALEIC ANHYDRIDE & & & SODIUM & & \\
1 3-PHENYLENEDIAMINE & 0.35 & & DIMETHYLDITHIOCARBAMATE & 0.35 & \\
\hline DI(2-ETHYLHEXYL) PHTHALATE & 0.35 & & NITRILOTRIACETIC ACID & 0.35 & \\
\hline 2 4-DICHLOROPHENOL & 0.35 & & BROMOCHLORODIFLUOROMETHANE & 0.35 & \\
\hline PROPIONALDEHYDE & 0.35 & & TOLUENE-2 4-DIISOCYANATE & 0.35 & \\
\hline 1 4-DIOXANE & 0.35 & & TITANIUM TETRACHLORIDE & 0.35 & \\
\hline
\end{tabular}

Table 24. Correctly identified chemicals for both Ergon and Clorox.

\begin{tabular}{|c|c|c|c|c|}
\hline \multirow[b]{2}{*}{ Facility } & \multirow[b]{2}{*}{ CAS } & \multirow[b]{2}{*}{ Chemical Name } & \multicolumn{2}{|c|}{ Inventory } \\
\hline & & & Max daily lb & Ave daily Ib \\
\hline \multirow[t]{4}{*}{ Ergon } & 67-64-1 & Acetone & $100-999$ & $100-999$ \\
\hline & 1310-73-2 & sodium hydroxide & $10,000-99,999$ & $10,000-99,999$ \\
\hline & $7647-01-0$ & $\begin{array}{l}\text { hydrogen } \\
\text { chloride }\end{array}$ & $10,000-99,999$ & $10,000-99,999$ \\
\hline & $108-88-3$ & Toluene & 1,000-9,999 & $100-999$ \\
\hline \multirow[t]{4}{*}{ Clorox } & $7664-93-9$ & sulfuric acid & 1,000-9,999 & $1,000-9,999$ \\
\hline & $67-63-0$ & propan-2-ol & $\begin{array}{l}100,000- \\
999,999\end{array}$ & $\begin{array}{l}100,000- \\
999,999\end{array}$ \\
\hline & $7439-92-1$ & Lead & $10,000-99,999$ & $10,000-99,999$ \\
\hline & 1310-73-2 & sodium hydroxide & $10,000-99,999$ & $10,000-99,999$ \\
\hline
\end{tabular}

Table 25. TIC chemicals in the Tier II report for Ergon not Identified by TIC-Master.

\begin{tabular}{|l|l|l|l|l|l|l|l|l|}
\hline CAS & Chemical Name & \multicolumn{3}{|l|}{ NFPAH/F/I } & \multicolumn{3}{|l|}{ ITF40 (HSC/HPC/RR) } \\
\hline $8052-42-4$ & $\begin{array}{l}\text { Asphalt, [at or above its flash } \\
\text { point] }\end{array}$ & & 1 & 0 & Negligible & Likely & Low \\
\hline $8002-26-4$ & Tall oil & 1 & & & Negligible & Occasional & Low \\
\hline $7783-06-4$ & hydrogen sulfide & 4 & 4 & 0 & Catastrophic & Occasional & High \\
\hline $8008-20-6$ & Kerosene & 2 & 2 & 0 & Marginal & Likely & Moderate \\
\hline $7727-37-9$ & Nitrogen, compressed & & & & Indeterminate & Frequent & Not Ranked \\
\hline $7664-38-2$ & phosphoric acid & 2 & & & Marginal & Likely & Moderate \\
\hline $16721-80-5$ & sodium hydrogen sulfide & & & & Indeterminate & Occasional & Not Ranked \\
\hline
\end{tabular}

For the sanitation product industry, 113 chemicals were estimated (Table 26). The Tier II report for Clorox Products Manufacturing Co. estimated 23 chemicals. Of these, nine appear in the TIC database, and four of these chemicals were actually identified. Tables 24 and 27 show the TICs identified and not identified, respectively. 
Table 26. Full listing of chemicals predicted for sanitation product manufacturing, with the first 30 ranked.

\begin{tabular}{|c|c|c|c|c|c|}
\hline CHEMICAL NAME & \%Prob & Rank & CHEMICAL NAME & \%Prob & Rank \\
\hline PHOSPHORIC ACID & 41.56 & 1 & FORMIC ACID & 1.11 & \\
\hline HYDROCHLORIC ACID & 24.67 & 2 & N-BUTYL ALCOHOL & 1.11 & \\
\hline SODIUM HYDROXIDE (SOLUTION) & 22.89 & 3 & METHYL ISOBUTYL KETONE & 1.11 & \\
\hline 11 1-TRICHLOROETHANE & 21.33 & 4 & PHENOL & 1.11 & \\
\hline SULFURIC ACID & 18.89 & 5 & 12-DICHLOROBENZENE & 0.89 & \\
\hline METHANOL & 17.33 & 6 & CUMENE & 0.89 & \\
\hline DICHLOROMETHANE & 15.56 & 7 & BUTYL ACRYLATE & 0.89 & \\
\hline AMMONIA & 13.33 & 8 & CRESOL (MIXED ISOMERS) & 0.89 & \\
\hline ETHYLENE GLYCOL & 10.67 & 9 & CHROMIUM & 0.89 & \\
\hline NITRIC ACID & 10.22 & 10 & SEC-BUTYL ALCOHOL & 0.67 & \\
\hline CHLORINE & 10.00 & 11 & PERACETIC ACID & 0.67 & \\
\hline DIETHANOLAMINE & 9.11 & 12 & METHYL METHACRYLATE & 0.67 & \\
\hline XYLENE (MIXED ISOMERS) & 8.44 & 13 & PHTHALIC ANHYDRIDE & 0.67 & \\
\hline TOLUENE & 6.89 & 14 & BUTYL BENZYL PHTHALATE & 0.67 & \\
\hline TETRACHLOROETHYLENE & 6.89 & 15 & 2-ETHOXYETHANOL & 0.67 & \\
\hline ISOPROPYL ALCOHOL & 5.33 & 16 & ETHYL ACRYLATE & 0.67 & \\
\hline SODIUM NITRITE & 5.33 & 17 & MERCURY & 0.67 & \\
\hline METHYL ETHYL KETONE & 5.11 & 18 & NICKEL & 0.67 & \\
\hline 12 4-TRIMETHYLBENZENE & 5.11 & 19 & THIOUREA & 0.44 & \\
\hline ACETONE & 4.67 & 20 & BENZENE & 0.44 & \\
\hline DIBUTYL PHTHALATE & 4.67 & 21 & TRICHLOROFLUOROMETHANE & 0.44 & \\
\hline N-METHYL-2-PYRROLIDONE & 4.67 & 22 & DICHLORODIFLUOROMETHANE & 0.44 & \\
\hline HYDROGEN FLUORIDE & 4.67 & 23 & ACRYLIC ACID & 0.44 & \\
\hline TRICHLOROETHYLENE & 4.44 & 24 & CHLOROACETIC ACID & 0.44 & \\
\hline FORMALDEHYDE & 4.00 & 25 & DIETHYL PHTHALATE & 0.44 & \\
\hline FREON 113 & 3.78 & 26 & M-XYLENE & 0.44 & \\
\hline 14-DICHLOROBENZENE & 3.56 & 27 & PROPYLENE & 0.44 & \\
\hline NAPHTHALENE & 2.67 & 28 & HYDROQUINONE & 0.44 & \\
\hline ALUMINUM OXIDE (FIBROUS FORMS) & 2.67 & 29 & 14-DIOXANE & 0.44 & \\
\hline 2-PHENYLPHENOL & 2.22 & 30 & ALUMINUM (FUME OR DUST) & 0.44 & \\
\hline N-HEXANE & 2.22 & 31 & MANGANESE & 0.44 & \\
\hline 11-DICHLORO-1-FLUOROETHANE & 2.22 & & DICHLOROBENZENE (MIXED ISOMERS) & 0.44 & \\
\hline STYRENE & 1.78 & & PIPERONYL BUTOXIDE & 0.22 & \\
\hline ETHYLBENZENE & 1.56 & & URETHANE & 0.22 & \\
\hline NITRILOTRIACETIC ACID & 1.33 & & CARBON TETRACHLORIDE & 0.22 & \\
\hline
\end{tabular}




\begin{tabular}{|c|c|c|c|c|c|}
\hline CHEMICAL NAME & \%Prob & Rank & CHEMICAL NAME & \%Prob & Rank \\
\hline BIS(TRIBUTYLTIN) OXIDE & 0.22 & & DIPHENYLAMINE & 0.22 & \\
\hline CHLOROMETHANE & 0.22 & & $\begin{array}{l}\text { POTASSIUM } \\
\text { DIMETHYLDITHIOCARBAMATE }\end{array}$ & 0.22 & \\
\hline CARBON DISULFIDE & 0.22 & & SODIUM DIMETHYLDITHIOCARBAMATE & 0.22 & \\
\hline ETHYLENE OXIDE & 0.22 & & CAPTAN & 0.22 & \\
\hline DICYCLOPENTADIENE & 0.22 & & 2-MERCAPTOBENZOTHIAZOLE & 0.22 & \\
\hline CUMENE HYDROPEROXIDE & 0.22 & & 1 2-DICHLOROETHYLENE & 0.22 & \\
\hline O-TOLUIDINE & 0.22 & & C.I. BASIC GREEN 4 & 0.22 & \\
\hline ACETOPHENONE & 0.22 & & TOLUENE-2 4-DIISOCYANATE & 0.22 & \\
\hline BENZYL CHLORIDE & 0.22 & & POLYCHLORINATED BIPHENYLS & 0.22 & \\
\hline METHYLENEBIS(PHENYLISOCYANATE) & 0.22 & & $\begin{array}{l}\text { 1-(3-CHLOROALLYL)-3 } 5 \text { 7-TRIAZA-1- } \\
\text { AZONIAADAMANTANE CHLORIDE }\end{array}$ & 0.22 & \\
\hline 4 4'-METHYLENEDIANILINE & 0.22 & & LEAD & 0.22 & \\
\hline P-XYLENE & 0.22 & & ANTIMONY & 0.22 & \\
\hline ACRYLONITRILE & 0.22 & & CADMIUM & 0.22 & \\
\hline VINYL ACETATE & 0.22 & & COPPER & 0.22 & \\
\hline CYCLOHEXANOL & 0.22 & & TETRAMETHRIN & 0.22 & \\
\hline 2-METHYLPYRIDINE & 0.22 & & PHOSPHORUS (YELLOW OR WHITE) & 0.22 & \\
\hline 2-METHOXYETHANOL & 0.22 & & $\begin{array}{l}\text { TOLUENE DIISOCYANATE (MIXED } \\
\text { ISOMERS) }\end{array}$ & 0.22 & \\
\hline CYCLOHEXANE & 0.22 & & PERMETHRIN & 0.22 & \\
\hline PROPOXUR & 0.22 & & TRICLOPYR TRIETHYLAMMONIUM SALT & 0.22 & \\
\hline DI(2-ETHYLHEXYL) PHTHALATE & 0.22 & & FENARIMOL & 0.22 & \\
\hline N-DIOCTYL PHTHALATE & 0.22 & & HYDRAMETHYLNON & 0.22 & \\
\hline TRIETHYLAMINE & 0.22 & & & & \\
\hline
\end{tabular}

Table 27. TIC chemicals in the Tier II report for Clorox not identified by TIC-Master.

\begin{tabular}{|l|l|l|l|l|l|l|l|l|}
\hline CAS & Chemical Name & \multicolumn{2}{l|}{$\begin{array}{l}\text { NFPA } \\
\text { H/F/I }\end{array}$} & \multicolumn{2}{l|}{ ITF40 (HSC/HPC/RR) } \\
\hline $68608-26-4$ & $\begin{array}{l}\text { Sulfonic acids, petroleum, } \\
\text { sodium salts }\end{array}$ & & & & Indeterminate & Indeterminate & Not Ranked \\
\hline $8052-41-3$ & Stoddard solvent & 2 & 2 & 0 & Marginal & Indeterminate & Not Ranked \\
\hline $57-55-6$ & propane-1,2-diol & 1 & 1 & 0 & Negligible & Occasional & Low \\
\hline $151-21-3$ & sodium dodecyl sulphate & 2 & & & Marginal & Occasional & Moderate \\
\hline $61790-12-3$ & Fatty acids, tall-oil & & & & Indeterminate & Indeterminate & Not Ranked \\
\hline $65-85-0$ & Benzoic acid & 4 & 1 & 0 & Catastrophic & Occasional & High \\
\hline
\end{tabular}




\section{Most likely chemicals}

Tables 23 and 26 list the first 30 chemicals from each facility ranked in order of most likely. Reviewing the ranking of the first 30 chemicals for the chemical refining industry, it is interesting that even the 3oth chemical has a percent probability over $25 \%$. This seems to indicate that this type of facility would likely have a relatively high number of chemicals. In fact, the facility had 44 chemicals reported in its Tier II report.

\section{Most significant chemical hazards}

Tables 28 and 29 show refinery chemicals with flammability and health indices of 4 .

Tables 30 and 31 show that chemicals from cleaning products manufacturing had flammability (NFPA-F) and health (NFPA-H) indices of 4.

Table 28. Refinery chemicals with flammability indices of 4.

\begin{tabular}{|l|l|l|l|l|}
\hline NFPA_F & CAS & Chemical Name & \%Prob & NFPA_H \\
\hline 4 & 74908 & HYDROGEN CYANIDE & 13.33 & 4 \\
\hline 4 & 302012 & HYDRAZINE & 2.46 & 4 \\
\hline 4 & 75150 & CARBON DISULFIDE & 26.67 & 3 \\
\hline 4 & 75218 & ETHYLENE OXIDE & 1.40 & 3 \\
\hline 4 & 75569 & PROPYLENE OXIDE & 1.05 & 3 \\
\hline 4 & 124403 & DIMETHYLAMINE & 0.70 & 3 \\
\hline
\end{tabular}

Table 29. Refinery chemicals with health indices of 4.

\begin{tabular}{|l|l|l|l|l|}
\hline NFPA_H & CAS & Name & \%prob & NFPA_F \\
\hline 4 & 71432 & BENZENE & 78.95 & 3 \\
\hline 4 & 74908 & HYDROGEN CYANIDE & 13.33 & 4 \\
\hline 4 & 79107 & ACRYLIC ACID & 0.70 & 2 \\
\hline 4 & 79210 & PERACETIC ACID & 0.35 & 2 \\
\hline 4 & 91225 & QUINOLINE & 0.70 & 2 \\
\hline 4 & 107028 & ACROLEIN & 1.05 & 3 \\
\hline 4 & 107131 & ACRYLONITRILE & 0.35 & 3 \\
\hline 4 & 107186 & ALLYL ALCOHOL & 0.70 & 3 \\
\hline 4 & 108952 & PHENOL & 39.30 & 2 \\
\hline 4 & 120832 & 2 4-DICHLOROPHENOL & 0.35 & 1 \\
\hline 4 & 123319 & HYDROQUINONE & 0.70 & 1 \\
\hline 4 & 302012 & HYDRAZINE & 2.46 & 4 \\
\hline
\end{tabular}




\begin{tabular}{|l|l|l|l|l|}
\hline NFPA_H & CAS & Name & \%prob & NFPA_F \\
\hline 4 & 7439976 & MERCURY & 20.70 & i \\
\hline 4 & 7440439 & CADMIUM & 3.86 & $\mathrm{i}$ \\
\hline 4 & 7632000 & SODIUM NITRITE & 3.51 & $\mathrm{i}$ \\
\hline 4 & 7647010 & HYDROCHLORIC ACID & 34.04 & 0 \\
\hline 4 & 7664393 & HYDROGEN FLUORIDE & 22.81 & 0 \\
\hline 4 & 7664939 & SULFURIC ACID & 61.75 & 0 \\
\hline 4 & 7697372 & NITRIC ACID & 1.40 & 0 \\
\hline 4 & 7723140 & PHOSPHORUS (YELLOW OR WHITE) & 1.75 & $\mathrm{i}$ \\
\hline 4 & 7726956 & BROMINE & 0.35 & $\mathrm{i}$ \\
\hline 4 & 7782414 & FLUORINE & 1.05 & 0 \\
\hline 4 & 7782505 & CHLORINE & 53.68 & 0 \\
\hline
\end{tabular}

Table 30. Cleaning product chemicals with flammability indices of 4 or higher.

\begin{tabular}{|l|l|l|l|l|}
\hline NFPA F & CAS & Name of Chemical & $\%$ Prob & NFPA H \\
\hline 4 & 75150 & CARBON DISULFIDE & 0.22 & 3 \\
\hline 4 & 75218 & ETHYLENE OXIDE & 0.22 & 3 \\
\hline 4 & 74873 & CHLOROMETHANE & 0.22 & 2 \\
\hline 4 & 115071 & PROPYLENE & 0.44 & 1 \\
\hline
\end{tabular}

Table 31. Cleaning product chemicals with health indices of 4 or higher (i indicates insufficient data).

\begin{tabular}{|l|l|l|l|l|}
\hline NFPA H & CAS & Name & $\%$ Prob & NFPA F \\
\hline 4 & 56359 & BIS(TRIBUTYLTIN) OXIDE & 0.22 & $\mathrm{i}$ \\
\hline 4 & 71432 & BENZENE & 0.44 & 3 \\
\hline 4 & 79107 & ACRYLIC ACID & 0.44 & 2 \\
\hline 4 & 79210 & PERACETIC ACID & 0.67 & 2 \\
\hline 4 & 107131 & ACRYLONITRILE & 0.22 & 3 \\
\hline 4 & 108952 & PHENOL & 1.11 & 2 \\
\hline 4 & 123319 & HYDROQUINONE & 0.44 & 1 \\
\hline 4 & 7439976 & MERCURY & 0.67 & $\mathrm{i}$ \\
\hline 4 & 7440439 & CADMIUM & 0.22 & $\mathrm{i}$ \\
\hline 4 & 7632000 & SODIUM NITRITE & 5.33 & $\mathrm{i}$ \\
\hline 4 & 7647010 & HYDROCHLORIC ACID & 24.67 & 0 \\
\hline 4 & 7664393 & HYDROGEN FLUORIDE & 4.67 & 0 \\
\hline 4 & 7664939 & SULFURIC ACID & 18.89 & 0 \\
\hline 4 & 7697372 & NITRIC ACID & 10.22 & 0 \\
\hline 4 & 7723140 & PHOSPHORUS (YELLOW OR WHITE) & 0.22 & $\mathrm{i}$ \\
\hline 4 & 7782505 & CHLORINE & 10.00 & 0 \\
\hline
\end{tabular}


Finally, the following chemicals were given as part of the exercise:

- 8002-26-4 tall oil: F1, H1

- 7783-06-4 hydrogen sulfide: $\mathrm{F}_{4}, \mathrm{H}_{4}$

- 64741-44-2 diesel: not in database (F2, Ho)

- 8008-20-6 kerosene: F2, H2

- 80o6-61-9 gasoline: $\mathrm{F}_{3}, \mathrm{H} 1$

- 68608-26-4 hostapur: F1, H2

- 57-55-6 propylene glycol: F1, H1

- 65-85-o benzoic acid: F1, H4

- 8002-09-3 terpene alcohol: not in database (F1, H1)

The three greatest hazards were determined:

1. Hydrogen cyanide, which had NFPA-H $=4$ and NFPA-F $=4$. Associated with refining.

2. Hydrogen sulfide, which also had an NFPA-H $=4$ and an NFPA-F $=4$.

3. Benzene: NFPA-H $=4$ and NFPA-F $=3$. Associated with both industries.

Once again, strong cases could be made for other chemicals.

Recommendations were made using the information in the database, the SOPs, and professional judgment. Fire protection equipment and breathing protection are recommended, particularly self-contained breathing apparatus for those who may have to enter the area while fires from the suicide bomber explosions are burning. For $\mathrm{HCN}$ releases, level A protection is strongly recommended, including use of SCBA, at least until testing verifies safe levels. For organic vapors (benzene), SCBA or organic vapor cartridges in air purifying respirators are recommended. Level B protection (Tyvek) should be sufficient, but Level A could be warranted for highly contaminated areas. Of course, this protection is provided on the order of SCBA and the need for test results might not be practical for a military operation. This would make other courses of action more attractive.

Release modeling

A model run was conducted evaluating the release of 200 gal of hydrogen cyanide ( $\mathrm{HCN}$ ) from the refinery. Figure 21 is an aerial photo showing the release. 


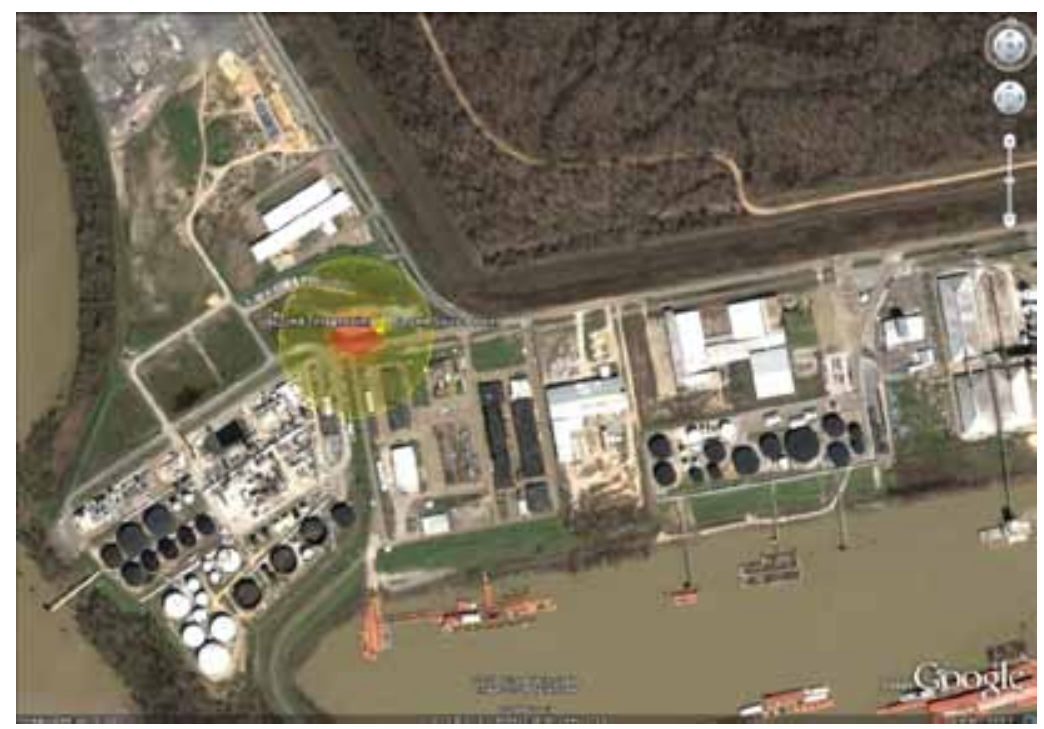

Figure 21. Modeled release of $\mathrm{HCN}$.

Response to environmental question

The test team provided a response to the environmental question, "Can you address community concerns about 7783-06-4 Hydrogen sulfide concentrations in a nearby waterway?" The response reads,

Hydrogen sulfide $\left(\mathrm{H}_{2} \mathrm{~S}\right)$ generally exists as a gas. However, it can dissolve in water. It rarely will cause a health concern in water, but can make it unpalatable as it can impart a foul taste (rotten egg) in the water. Obviously, a terrorist attack would be an extraordinary circumstance that could affect normal access to utilities, such as drinking water. Because of the issues with $\mathrm{H}_{2} \mathrm{~S}$ and with other, even more toxic chemicals, we would recommend that those residents who are not evacuated be put on bottled potable water. If the waterway is contaminated, a cleanup would be instituted. For $\mathrm{H}_{2} \mathrm{~S}$, which is a highly reduced chemical, the easiest removal mechanism would be aeration, which would drive the gas out of solution or oxidize the sulfide into sulfate forms.

\section{Test 5 summary}

\section{Scenario}

The Test 5 scenario assumes that U.S. Armed Forces want to select a location for an operation. Two sites are under consideration. Intelligence has confirmed the presence and volumes of the chemicals listed. Armed resistance is expected if either site is acquired or maintained. The test 
team was asked to identify the preferred location and state the basis for the recommendation. The evaluation should include the potential impact to municipal facilities like parks, schools, fire stations, hospitals, etc.

(Figure 22).

Test_ $\underline{5}$ Scenario

Date:12/03/09 Time Submitted:_0915 CST

\begin{tabular}{l|l} 
Industrial Processes or & $\begin{array}{l}\text { TIC Release Mechanism: } \\
\text { Process Name: } \\
\text { select a location for an } \\
\text { operation. Two sites are } \\
\text { under consideration. } \\
\text { Intelligence has confirmed } \\
\text { the presence of the } \\
\text { chemicals listed below. } \\
\text { Armed resistance is } \\
\text { expected if either site is } \\
\text { acquired or maintained. }\end{array}$ \\
\hline
\end{tabular}

TICs:

Site A: Chemicals include 143-33-9 Sodium Cyanide (100-999 lbs), 151-50-8 Potassium Cyanide (1,000-9,999

lbs), and 7664-93-9 Sulfuric Acid (1,000-9,999 lbs). These

volumes are the average daily amounts.

Note: This is a complete chemical list.

Site B: Chemicals include 7647-01-1 Muriatic Acid (1,000-9,999 lbs); 7664-93-9 Sulfuric Acid (1,000-9,999

lbs); 8006-64-2 steam distilled turpentine (1,000-9,999

lbs); 1310-73-2 sodium hydroxide(10,000-99,999 lbs);

25155-23-1 trixylenylphosphate (1,000-9,999 lbs); 8002-

26-4 Tall oil (1,000-9,999 lbs); 7704-34-9 sulfur(1,000-

9,999 lbs); and 7681-52-9 sodium hypochlorite (1,000-

$9,999 \mathrm{lbs})$. These volumes are the average daily amounts.

Note: This is only a partial chemical list.

Site Designation:

Single Site:

Multiple Sites: $\underline{X}$

General Location*:

Site $A^{*}$ :

Longitude: $90^{\circ} 48^{\prime} 32.31^{\prime \prime}$

W

Latitude: $32^{\circ} 22^{\prime} 37.36^{\prime \prime} \mathrm{N}$

Elevation:
Atmospheric conditions:

Temperature: $39 \mathrm{deg} \mathrm{F}$

Wind Speed: $15 \mathrm{mph}$

Wind Direction: West

Precipitation: None

Other Potential Hazards:

Other Characteristics: Identify the preferred location and state the basis for the recommendation. Include in your evaluation the potential impact to municipal facilities like parks, schools, fire stations, hospitals, etc.

* See the Other Characteristics section. For example, the U.S. Army may wish to occupy a 2-square-mile area centered at the location provided by Site A*.

Figure 22. Scenario description for Test 5. 


\section{Results}

Response time

The estimated working time was just over $5 \mathrm{hr}$.

\section{Facility information}

This test was significantly different than the previous tests in two ways. First, the given locations did not correspond to the real facility. The Site A coordinates refer to the Pecan Ridge Apartment Complex at 2501 Culkin Road, Vicksburg, MS (Latitude (Lat) $32^{\circ} 22$ '37.36”N; Longitude (Long) $90^{\circ} 48^{\prime} 32.31$ 'W). This complex is just east of River Region Hospital and Sherman Avenue Elementary School. The Site B coordinates refer to a steel facility at 1250 Highway 27 , Vicksburg, MS (Lat $32^{\circ} 19^{\prime} 54.29 " \mathrm{~N}$; Long $\left.90^{\circ} 50^{\prime} 13.86 " \mathrm{~W}\right)$. A second departure was that an NAICS code was not provided. Rather, a listing of chemicals was provided for the two facilities. The team needed to determine the industry from the chemicals provided. The test team estimated that Site A was a metal working facility with an NAICS code ranging from 331000 to 333000 . In particular, the team guessed that the facility was a rolled steel shape manufacturer (3331221). This response was accurate. The test team's response proposed that Site B was a paper processing facility: a paper mill (322121) or a paperboard mill (322130), based on the presence of Kemira ama-1750, which is a biocide specific to paper products and the combination of flotation agents, bleach, fuel, and acids/bases. This was also correct. Figure 23 is a location map.

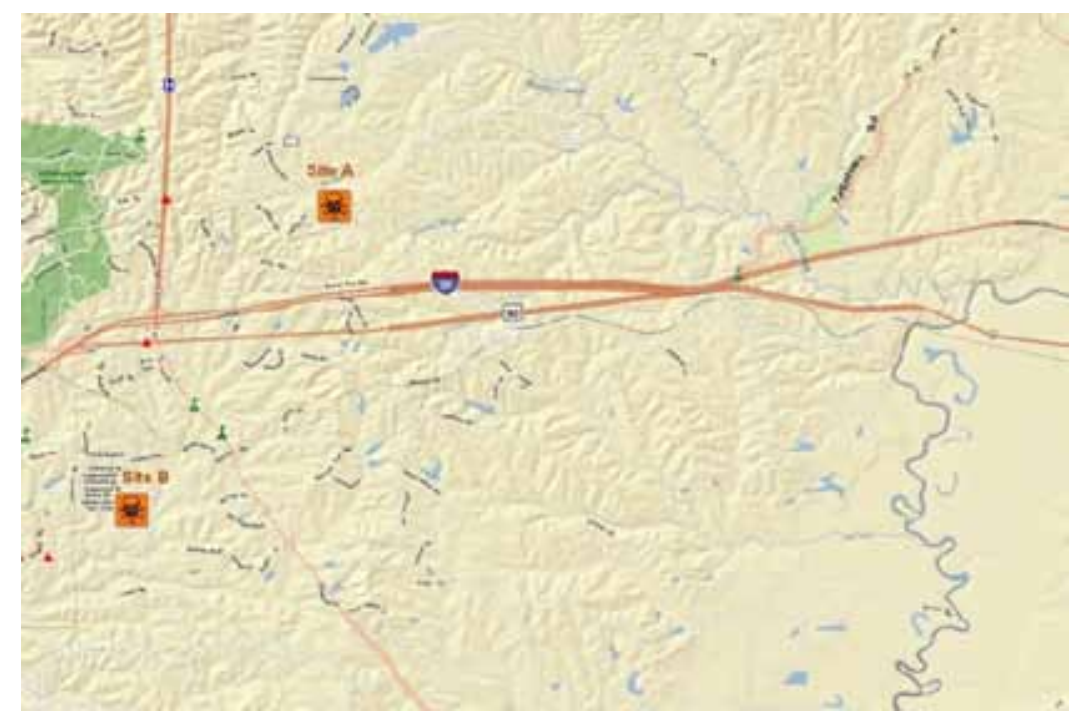

Figure 23. Location map for Test 5. 


\section{Chemical identification}

Chemicals for this exercise were provided (see Figure 22). Table 32 summarizes the chemicals and their risks.

Table 32. Chemicals from Sites A and B for Test 5.

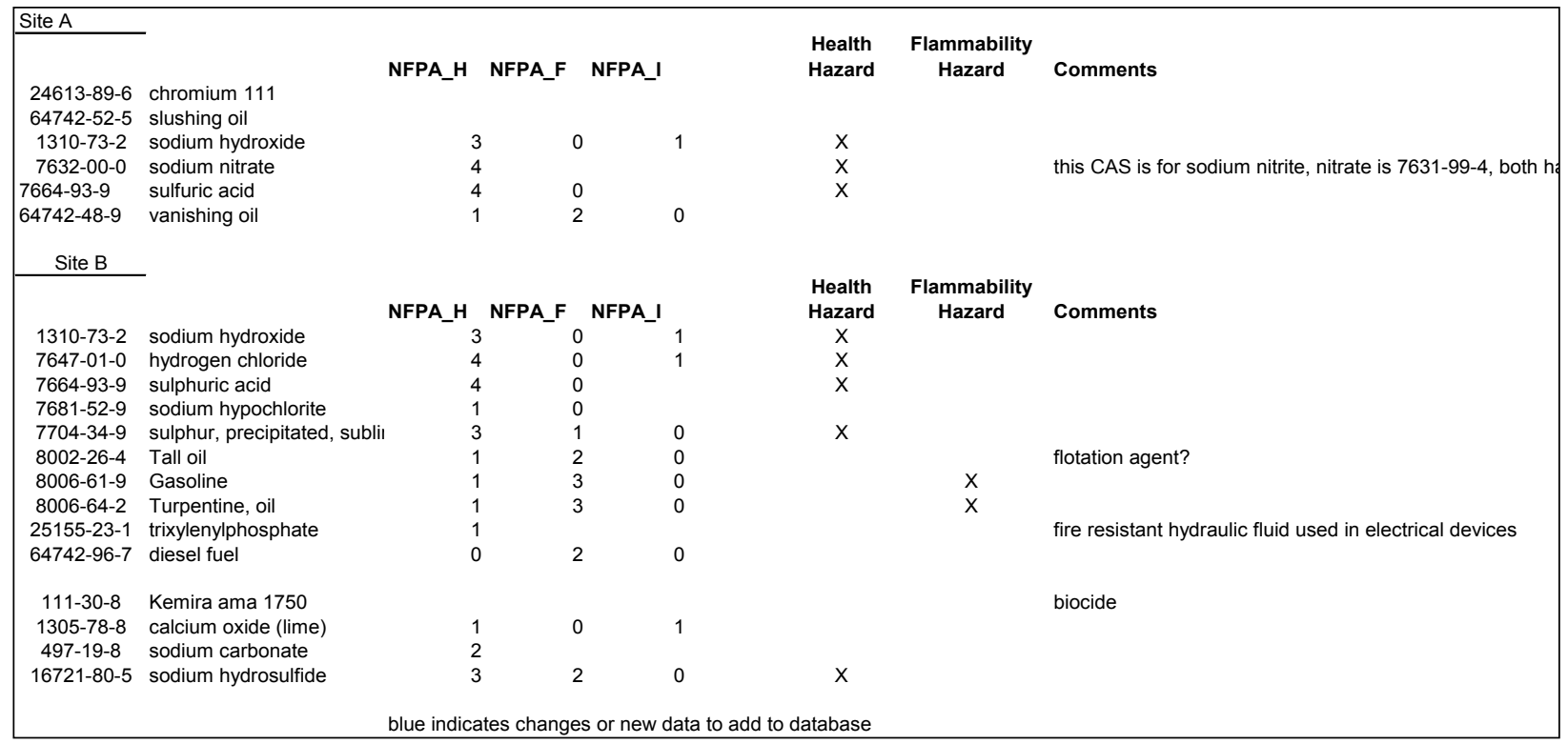

\section{Chemical hazards}

Site A has two chemicals with a health risk of 4 and one with a health risk of 3 :

- Sodium nitrate

- Sulfuric acid

Site A does not have any chemicals with flammability of 3 or higher.

Site B also has two chemicals with a health risk of 4 and three chemicals with a health risk of 3 :

- Hydrogen chloride (4)

- Sulfuric acid (4)

- Sodium hydroxide (3)

- $\quad$ Sulfur, precipitated (3)

- Sodium hydrosulfide (3)

Site B had no chemicals with a flammability hazard of 4 , but had two chemicals with flammability hazards of 3 : 
- Gasoline

- Turpentine oil

Release modeling

Figure 24 shows the results of a simulation of sulfuric acid releases from both facilities. The volume of release from both sites was 99,999 lb. Wind velocity and direction were given in the exercise.

Observations from the GIS data:

Site A:

1. The wind is blowing from the west; therefore, the resulting plume will most likely drift eastward, and continue to follow the dominant wind direction.

2. From the predicted plume resulting at Site A, the population potentially to be affected is approximately 1,638 people (using the census feature provided by the GIS system).

3. One school is present within the predicted plume (see green marker). No other facilities were identified.

Site B:

1. The wind is blowing from the west; therefore, the resulting plume will most likely drift eastward, and continue to follow the dominant wind direction.

2. From the predicted plume resulting at Site A, the population potentially to be affected is approximately 888 people.

3. None of the critical infrastructure (school, hospital, fire station, or police) is found in the predicted plume for site B (Figure 24a). However, based on the map, one can state that if the wind direction changes from "from west" to "from south," then three schools just northeast of Site B are affected, and these are quite close, closer in fact than the school affected in Site A.

Best site to perform operation

Analysis of chemical inventory. Based on the chemical inventory provided, Site B appears to have the most hazards. It has more chemicals, a higher total quantity of chemicals, more chemicals with health hazards of 3 or 4 , and more chemicals with flammability hazards. This would make Site A a better choice to conduct the operation. 


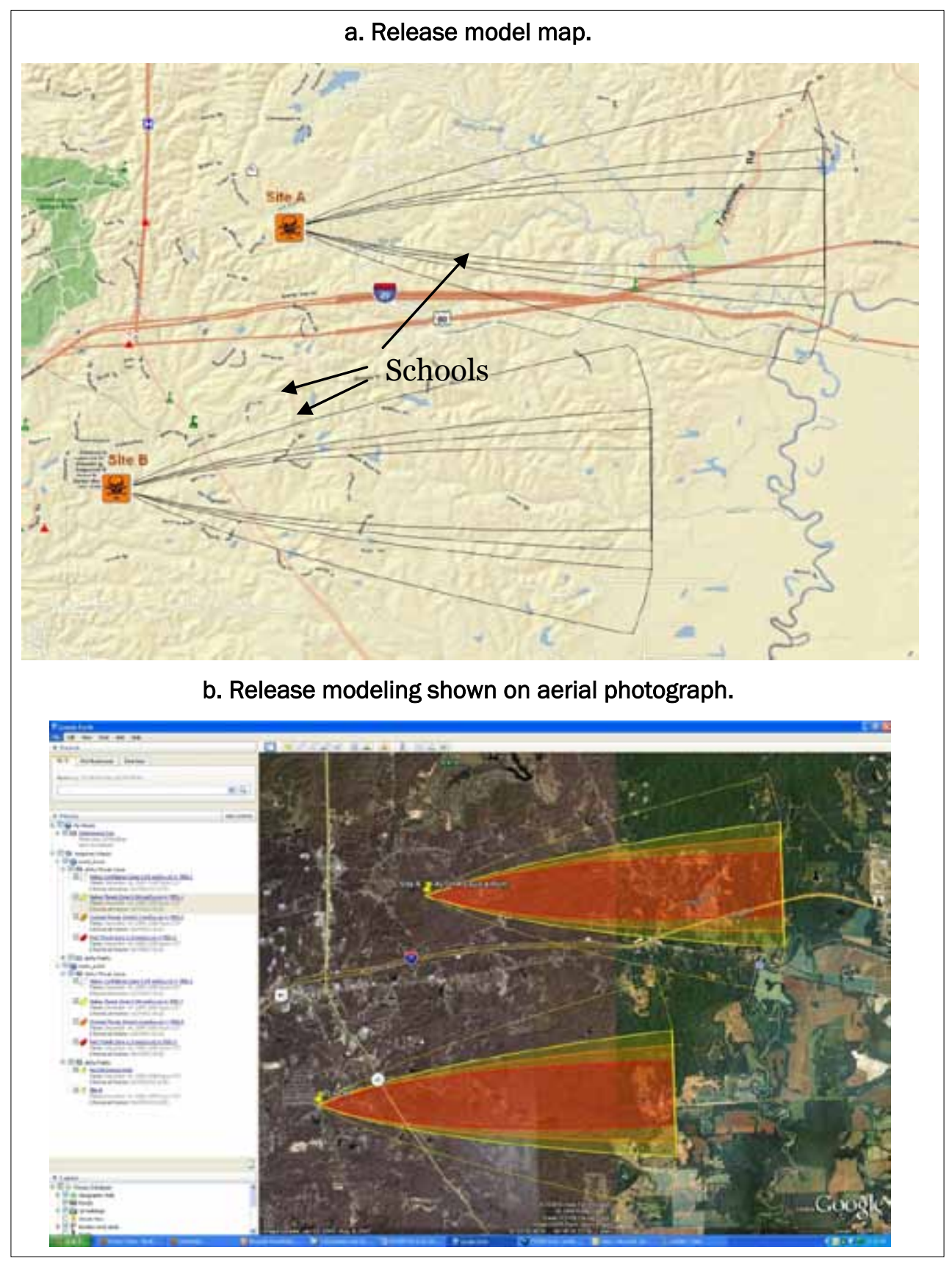

Figure 24. Maps of releases from two facilities from Test 5 scenario.

Assessment of release scenario. Site B would affect fewer people than Site A and would not affect any schools, compared to one school affected by Site A. However, this can change if the wind direction changes. The plume from Site A would cross Interstate 20, a major transportation highway. The plume from Site B would cross highway 27 , which is not nearly as busy.

Final assessment. Overall, Site B is recommended as the best choice to conduct the operation. If a release should occur, Site B should affect a 
smaller population and should not impact schools. There are some caveats; there are two schools close to site B that could be affected if the wind should change direction and Site B involves more chemicals that could be problematic. Chemical information and modeling can greatly aid in choosing the site. 


\section{Discussion}

\section{Response time}

The response time of $2 \mathrm{hr}$ was only met for one of the tests: Test 2. Response times ranged from 2 to more than $5 \mathrm{hr}$. The overall average response time was close to $3 \mathrm{hr}$. Response times were affected by the fact that the test team included participants from three different locations and that, due to conflicting schedules, they were not always able to work together at the same time. Of course, similar issues would likely occur in using the TIC-Master program during an actual scenario.

\section{Facility identification}

For Tests 1-4, the NAICS code was provided. In each case, the proper facility type was derived from the code, which is entirely expected. For Test 5 , complete chemical lists were provided for two facilities, but the type of facility was not. The team was able to determine the facility. However, for both facilities, there were distinctive chemicals that greatly aided the team in identification. It is clear that determining chemical facilities from a partial list is challenging due to the fact that most chemicals can be used for a wide range of industrial processes.

\section{Chemical prediction}

\section{Comparison of total number of chemicals}

Table 33 summarizes the chemicals predicted for Tests 1-4 (in Test 5 , the chemical list was provided). First, it is clear that the database provided more chemicals than those actually found at the site, ranging from a factor of slightly less than 2 (for Test 3) to more than 5 (Test 2). This reflects variability of chemicals used by similar industrial processes by different facilities. Analyzing possible chemicals from a particular industrial facility based on chemicals used by similar industries will likely lead to this overestimation. The challenging aspect of this technique is that the decision maker may have to deal with extraneous information that makes hazard estimation more cumbersome. 
Table 33. Scenario summary.

\begin{tabular}{|l|l|l|l|l|}
\hline Scenario & $\begin{array}{l}\text { \# TICs } \\
\text { Predicted }\end{array}$ & Tier II Report & $\begin{array}{l}\text { Tier II TICs (In } \\
\text { TMaster DB) }\end{array}$ & $\begin{array}{l}\text { Predicted TICs } \\
\text { Using TMaster } \\
\text { DB }\end{array}$ \\
\hline 1 & 156 & 58 & 35 & $15 / 35$ \\
\hline 2 & 26 & 5 & 4 & $2 / 4$ \\
\hline 3 & 91 & 53 & 30 & $15 / 30$ \\
\hline 4 Site A & 158 & 44 & 11 & $4 / 11$ \\
\hline 4 Site B & 113 & 23 & 9 & $4 / 9$ \\
\hline
\end{tabular}

\section{TIC chemical predictions versus chemicals actually reported}

Table 33 indicates that the total chemicals reported in the Tier II reports of the various industries were more than those commonly found in the database. As discussed in Chapter 2, the database developed for this program was inclusive of TICS defined by the combined lists of the ITF-40 (which included chemicals listed in ITF-25), and EPA TRI. Chemicals in common with the TIC-Master database can be definitively called TICs. However, TICs do not encompass all hazardous chemicals. The Tier II report had some hazardous chemicals not defined as TICs, as was expected. Expanding the list to incorporate other chemicals would probably not be too difficult. Additional chemical lists are readily available. The VFD indicated, for example, that programs like the Computer Aided Management of Emergency Operations (CAMEO - which focuses on releases, but does not have TIC-Master's predictive effort) lists 5000 to 7000 chemicals (National Oceanic and Atmospheric Administration (NOAA), undated). However, expanding the chemical list could also result in a similar increase of total chemicals reported, which was up to a factor of 5 (see Chapter 5). It is not clear if expanding the chemical would actually improve the ability to assess the risk at a given site, at the expense of information overload. A data gap analysis could be useful to determine if the addition of more chemicals would improve the system performance (Dortch et al. 2005).

Links could easily be developed to existing databases that would allow for a wider range of chemicals as well as allow users to check chemical data and recommendations from various sources. Some useful databases include:

- Wireless Information System for Emergency Responders (WISER) (http://wiser.nlm.nih.gov/)

- CAMEO (http://cameochemicals.noaa.gov/) 
- Chemical Biological Response Aid (CoBRA)

(http://www.defensegroupinc.com/cobra/)

- ALOHA (http://www.chemicalspill.org/OffSite/aloha.html).

Of these, ALOHA was the only database linked to TIC-Master, but the other databases could also be linked.

Table 33 also compares the defined TICs found at the site with those predicted by the TIC-Master program. The program identified about 50\% of the correct TICs. This must result from deficiencies in the industrychemical crosswalk. The relationships used to develop the crosswalk were found using the EPA TRI database. As mentioned in Chapter 2, this database uses a more limited list (less than 600 chemicals) compared to the combined list used in the TIC-Master database (more than 2000). Furthermore, the TRI focuses on released chemicals. It is possible that industries may have chemicals that were not released, so they are not reported on the TRI searches.

The TRI database was the best tool found to develop industry-chemical relationships. However, a better tool could be available within the next 2 to 3 years. As mentioned in Chapter 1, Tier II reporting has been primarily a paper process. However, the EPA is now strongly encouraging electronic submission (USEPA 2010). This will likely lead to digitized records of the Tier II reports that can be searched and sorted. Such a database would likely improve an ICCW. However, it would also likely increase the total number of chemicals estimated, once again increasing the potential for information overload. One advantage of the existing TRI database is that it focuses on released chemicals, which are probably of most importance.

\section{Top 10 chemical comparison}

For scenarios 1-4, the test team predicted the top 10 chemicals on site. In Scenario 2, two of the top 10 most probable chemicals were included in the Tier II Report. However, in this test, there were only four total chemicals, and a third top 10 chemical (i.e., ammonia) was very similar to another Tier II chemical (i.e., aqueous ammonia). For the other tests, the top 10 chemicals were represented 50\% to $80 \%$ in the Tier II Reports. Using the ranking feature to identify most likely chemicals appears to be a very useful feature for focusing risk assessment. 


\section{Assessment of hazards/risks}

Testing lacked a systematic means of comparing the risks from the predicted list to the actual. However, by looking over the chemicals, reviewing the hazard information, and applying professional judgment, it was determined that the risks derived from the chemicals found in the predicted chemicals completely encompassed the risks from the actual chemicals in all cases. A systematic screening tool, possibly based on the principles of decision science, could be useful to allow a first screening of the chemicals identified, which can be numerous for certain industrial processes.

As noted in Table 1 (Chapter 2), the health hazard data used during the tests was derived initially from the ITF-40 data. However, some of these data were replaced by NFPA health rankings, especially in the cases where a refereed NFPA ranking was available and it had a value greater than ITF40. The health hazard listings for the tests were categorically labeled as NFPA, which is misleading. Although these values are frequently the same, they are not derived with the same criteria, so that the test results may involve mixed health rankings that were used to assess chemical risks. It is believed that this did not affect the performance of the TIC-Master to assess the hazards/risks because the test team used the health rankings in the TICMaster database to assign health rankings to actual chemicals for comparison. However, the next version of the database should correct this issue by being consistent with health hazard definition and labeling. Table 12 (Chapter 4) provides examples of the inconsistency: the health ranking for ammonia is " 3 ," which is an example of a higher refereed NFPA code replacing the ITF-40 value (1); methanol also is ranked " 3 ," which is an example of an ITF-40 value that is greater than a refereed NFPA value (1) and so remained in the database; aqueous ammonia is represented by "4," which is an example of a chemical that does not have a refereed NFPA ranking so the ITF-40 value remained in the database; and the health ranking for ethanol is "2," which is an example of a chemical with a refereed NFPA ranking matching the ITF-40 ranking.

\section{Release modeling}

ALOHA was chosen as the modeling program after discussions with the Vicksburg Fire Department. The program is freely available and is designed to be user-friendly. The program was found to work well for most simple explosive and atmospheric releases. 
However, ALOHA had some limitations. It is simple to use because it has preset release scenarios, such as tank sizes and configurations. It is not easy to model situations beyond these scenarios, nor is it easy to model multiple-component releases.

Perhaps the greatest limitation of ALOHA is that it focuses solely on atmospheric releases. Several of the scenarios involved facilities near streams and rivers. It would have been helpful to determine whether contaminant release would impact these water bodies and, if so, the effect.

The good news is that it is entirely possible to add release modeling programs to the GIS system. In fact, in many cases, these various programs can be used together as a basis of comparison. CAMEO is a program developed by the EPA that is capable of airborne release modeling. According to the VFD, they typically attempt to model a release using ALOHA first. If this proves insufficient, they will then use CAMEO. CAMEO also has capabilities to assist industries in tracking chemicals and using this information to prepare Tier II reports. However, CAMEO does not have surface release capabilities.

The Hazard Prediction and Assessment Capability model (HPAC) was developed by the Oak Ridge National Laboratory, and it couples atmospheric and surface depositional modeling capability, which would make it a good fit for this application. GeoRAMS, a program developed to estimate the effect of hazardous chemical spills, has four models integrated for air, soil, surface water, and pipe rupture (ERDC-EL 2005).

The Joint Effects Model (JEM, http://www.jpeocbd.osd.mil/packs/ Default.aspx?pg=1701) is a suite of models being developed for the DoD that will combine the best models for air, explosive, and surface water release of chemical weapons, nuclear and radiological agents, and TICs. This model will be an obvious choice to integrate with systems like the TIC-Master.

Surface water modeling programs commonly used by the Corps of Engineers include CASCade 2 Dimensional SEDiment (CASC2D-SED) (Johnson et al. 2008) and Gridded Surface/Subsurface Hydrologic Analysis (GSSHA). Both of these are distributed sources models and are capable of achieving excellent results for modeling contaminant movement in the environment. However, these models require a higher level of input data 
and require longer run times than HPAC, which is specifically designed for emergency situations.

In terms of the data, a drawback is the lack of quantity estimation capability. Therefore, the quantity for each release had to be estimated, unless it was provided by the test condition (equivalent to the intelligence available). One guideline is that a given chemical could be assumed to be available in quantities greater than the reporting threshold (generally $1000 \mathrm{~kg}$, although this can vary). Quantity estimation is discussed in the section that follows.

\section{Quantity estimation}

TIC-Master has the ability to estimate chemicals related to industrial processes. However, the TIC-Master system does not currently have the capability to estimate chemical quantities. One assumption could be made if a chemical is identified - it is likely at a quantity greater than its reporting limit. Other than that, no quantity information is provided.

Simply knowing the type of industry is not enough information to estimate quantity. However, first order quantification may be possible if some details of the facility are known. Information such as the size of the facility footprint, information on shipping capacity of the facility, or the number and size of certain critical chemical storage tanks could allow for order of magnitude estimates of chemical quantities. Developing these relationships requires a concerted effort to work through a range of industries, each of which may have different criteria that could be used to estimate quantity.

\section{Identification of industry}

For the first four exercises, either the NAICS code was provided or the industry name was provided, meaning that TIC-Master was used to determine the industry or NAICS code, respectively. Obviously, these are easily accomplished tasks. In test 5 , the team was tasked with identifying two industries from a list of chemicals from each facility. The task was accomplished, but it was challenging. Most chemicals were found to be of limited value in identifying an industry, or even in greatly narrowing down the possibilities, because they are so widely used. However, some industries have distinctive chemicals that could be very useful for quickly identifying the industry. 
Improved algorithms to identify industries from complete or partial chemical lists would be valuable. Identification of distinctive chemicals could be a key piece. However, evaluation of multiple chemicals could be valuable to narrow down possible industries.

The GIS system could also be a useful tool for industry identification. Tools could be developed to evaluate facility footprints obtained from aerial imagery and compare them to a library of footprints to look for similarities. Such a system would require considerable development to design, construct, and evaluate.

\section{Standard operating procedures (SOPs)}

SOPs had a limited role in the testing of the TIC-Master program. The SOPs were used to evaluate three chemicals in Test 1 (page 35), one in Test 2 (page 43), and provided information to address the environmental question in Test 4 (page 63). It is clear that the information provided was quite valuable in assessing risks and promoting mitigation. However, the effort to produce these SOP reports was substantial. Creating these reports for all the chemicals in ITF-40 would be costly. Future efforts might be better spent in other system enhancements.

\section{System modification recommendations based on test results}

After evaluating the results of five tests, concepts on improving TIC-Master for further use were developed. Recommendations resulting from the test observations are:

- Develop a chemicals-to-industry crosswalk to improve estimation of industries based on partial chemical lists. This should focus on the use of industry-distinctive chemicals whenever available.

- Make the system easier to use in general.

- Make it easier to add sites and define and redefine industry use.

- Increase the chemical database to contain other hazardous chemicals that are not defined as TICs.

- Develop a means of estimating volume of chemicals. This process might be challenging, but could use relationships based on facility size, number of storage tanks, etc.

- All TICs are a concern. However, some are particularly dangerous. Right now, the list is presented alphabetically. It would be useful to develop an alarm when the most hazardous materials are identified. 
- Address the importance of the health hazard rankings by defining and identifying the primary methods for determining the rankings and provide hazard ranking data for more chemicals, in particular for the TRI-based chemicals that comprise the chemical prediction subset.

- ALOHA is adequate for air modeling. Groundwater modeling would require too much data and probably would not be a factor to consider in the IPB process. However, a surface release model would enhance capabilities. A logical step would link the system to the new Army Joint Effects Model (JEM).

- Weave in identification of areas of concern, including topographic factors (such as streams, lakes), transportation factors (i.e., key roads, railroads), dense residential areas, and schools, hospitals, fire departments, etc. Test 5 touched on this by identifying schools and allowing searches for population counts in the plume area. However, it would be useful to automatically provide more details of these features.

\section{Feedback from possible user organizations (MANSCEN, the Army Intelligence Center, Army Geospatial Center, Program Manager for Army Geospatial Data Model)}

Various meetings were carried out with the Army Maneuver Support Center of Excellence (MSCoE), the Army Intelligence Center (AIC), the Army Geospatial Center (AGC) and the Program Manager for the Army Geospatial Data Model. MSCoE, AIG, and AGC were considered possible end users. AGC and PMAGDM were possible hosts for the program, or at least elements of the program. A fact sheet has been prepared to provide interested parties with information on the program (Appendix D).

\section{Army Intelligence Center}

The AIC was briefed on 8 April 2009 at Fort Huachuca, AZ. Kevin Enright of the AIC attended the project review on 27 May 2009. AIC identified the program as providing unique and important capabilities that can be used by the intelligence community. The AIC agreed with the assessment that the Global War on Terror, Operation Enduring Freedom, and Operation Iraqi Freedom indicate that operations in urban environments may be unavoidable, which brings into play issues with chemicals from industrial facilities. The AIC developed an analogy where chemical hazards could be analogous to geographical hazards, affecting the mobility of forces where they are encountered. Overall, the AIC was interested in and supported the development of TIC-Master. A support email by Mr. Enright stated "I do 
believe that what you are planning will be helpful for maneuver commanders by showing them a new aspect of the battlespace and its effect on friendly and enemy movement" (Appendix E). However, the AIC indicated that although TIC-Master output would be used in the IPB process, it would likely be operated by other units, such as engineers, chemical officers, or geospatial officers.

A key portion of the discussion focused on whether the system would benefit from classified sources. Without getting into specifics, classified information on various industrial facilities throughout the world is available. Tapping into these sources could enhance the value of the system. However, there are severe limitations to using a classified system that would hamper the effectiveness of TIC-Master if these sources were utilized. First, a secured area would have to be set up in the field to facilitate its use. Second, many National Guard and Reserve units do not have SIPRnet (the DoD internet that contains secret and top secret sources) resources to use or train with the system. Third, only the United Kingdom and Australia currently have access to the US SIPRnet system. Many coalitions include countries beyond these. Because of these limitations, it was decided to keep the system unclassified at this time. The AIC also indicated that a system that is unclassified to a point, but can draw upon classified information, is actually quite cumbersome.

As an unclassified system, it would still be important for proper security to be maintained. Fortunately, the Department of Defense and the Army have greatly enhanced their unclassified security capabilities. Use of passwords and controlled access cards (CAC) could provide sufficient security for unclassified use.

The AIC indicated that using ARCGIS as the program platform was a good choice and that the methods used would be easily integrated into Army operations.

\section{Feedback from MSCoE}

The MSCoE was determined to be a potential end user of TIC-Master program. Several interactions were conducted with MSCoE. On 27 April 2008, a workshop was conducted at Vicksburg, MS. MSCoE experts participated both in person and via teleconference. On 22 April 2009, a briefing was conducted to MSCoE at Fort Leonard Wood (FLW). On 08 June 2010, a phone meeting was conducted with the Experimentation 
Branch of MSCoE. On 15 June 2010, a phone briefing was conducted with various MSCoE units. And on 20-21 July 2010, the TIC-Master team conducted a series of meetings at FLW to demonstrate the TIC-Master program. The following conclusions were obtained from these meetings:

- The TIC-Master system offers unique capabilities in assessing chemicals and risks associated with industrial facilities.

- This capability cross cuts multiple Army capabilities (engineer, chemical, intelligence, geospatial). However, of these organizations, the engineer units would be most likely to use such a program.

- The system has potential value for battle plan preparation as it was originally conceived. However, its greatest value may in assessing base camp locations.

Although the Corps of Engineers would most likely utilize the program for planning, response to releases from an acute perspective is the responsibility of the Chemical Corps (Mauroni 2002). So, these units may also find the program very useful.

The following improvement areas were identified:

- Provide better and more accessible information on environmental fate, management, and response.

- Provide more information on personal protective equipment considerations. Particularly useful for the Army would be an assessment of whether standard issue protective equipment is suitable for protection.

- Provide links to resources for response to release of the contaminants. A particular resource discussed was The Emergency Response Guidebook (U.S. Department of Transportation (USDOT) 2008), which is available in electronic format. It would be even more useful if this resource could be automatically linked to specific chemicals. Links could also be provided to internet sources on the chemicals.

- Provide chemical lists for illegal facilities (i.e., drug manufacturing, bomb making).

- Provide multiple chemical and plume release capabilities.

Interestingly, a briefing to the Department of Public Works (DPW) generated substantial interest in the program. The DPW was interested in the concept of modifying the program to manage chemical risks at the Fort itself. The GIS and release modeling capabilities were determined as useful 
tools for both planning and reactive situations. A white paper has been prepared proposing a small program to modify the existing program for this application (Appendix F). Such a project could be a good segue into developing civilian applications (Chapter 5).

Overall, MSCoE determined that a product based on the TIC-Master platform could be very useful to several mission areas. Consequently, they provided a formal endorsement memorandum supporting the project (Appendix G).

\section{Interactions with the Army Geospatial Center}

Briefings were carried out at the Army Geospatial Center (AGC) at Fort Belvoir (Alexandria, VA) on 24 February 2009. Of particular interest was a briefing with the Program Manager of the Army Geospatial Data Model (PMAGDM), Kevin Backe. The AGDM is a collection of geospatial data whose maintainance is critical for the Army that is not maintained on other geospatial systems. The PMAGDM was not interested in the TIC-Master program as a whole, but rather focused on determining if the data model for the system was useful for the AGDM and if the data were in a format that could be imported. After briefing the PMAGDM and allowing Mr. Backe to review the data model, it was determined that the information contained in the system, particularly the ICCW, was unique and should be incorporated into the AGDM.

\section{Adaptations for civilian use}

As the project progressed, the topic of adaptation for civilian use was frequently discussed. Civilian organizations who contributed feedback included the VFD, the Mississippi Department of Environmental Quality, MEMA, disaster response groups from the Corps of Engineers Vicksburg District, and homeland security researchers at Applied Research Associates, Inc. The obvious difference with civilian applications is that the capability to estimate chemicals would not be required, as chemical information from EPA Tier 2 reports is generally available. However, features such as the ability to identify TIC facilities within an area, identify populations, roads, and various public facilities in the area, and to model releases could be of great value in various civilian applications. On a smaller scale, a system could be useful for a municipality or even a given industrial complex with multiple sites in a given area to track and manage their hazardous material. The release modeling features could be useful for training exercises as well 
as in an actual release response incident. TICs and other hazardous materials could be used by terrorists as a means of attack on the United States (Bennett 2003). From a police or homeland security perspective, the system could be useful for training and for scenario exercises to improve preparedness to terrorist or natural disaster incidents.

A key issue is importing chemical data into the system. Currently, EPA Tier II reports are not available electronically. However, as mentioned in Chapter 5, this is expected to change within the next 2 to 3 years. Once these data are available electronically, it should be possible to mine it for precise chemical information at a given facility.

Until that time, a stop gap measure could be the use of the existing TRI database, which, as discussed in Chapter 5, has some limitations, but does focus on chemicals most likely to be released. Another option would be to manually input information from EPA Tier II reports. This is obviously a cumbersome approach, but might be feasible for limited applications.

The ability to use a GIS system to locate and identify industrial facilities could be very beneficial during natural disaster response. Following Hurricane Katrina, many areas had nearly complete loss of signs, including road signs and business identification signs. This, as well as extensive facility damage, made it difficult to identify industrial facilities. The TICMaster system could be a valuable tool for these applications. 


\section{Conclusions}

This work unit produced an integrated GIS/database system called the TIC-Master program. The system was evaluated under five different scenarios. The response times ranged from 2 to $5 \mathrm{hr}$, averaging about $3 \mathrm{hr}$. The program was able to correctly identify the industries from the NAICS codes and manufacturing processes provided in the scenarios and was able to successfully predict two industries from the chemical lists provided in Scenario 5. TIC-Master listed 2 to 5 times more TICs than found in the EPA Tier II report for the facilities. In general, TIC-Master precisely identified about $50 \%$ of TIC chemicals actually reported. However, the chemical risks appeared to be encompassed by the chemicals that were identified. Narrowing the chemical list down by chemical frequency (in this case, using a 10 most likely list), helped to focus the predicted results. ALOHA was an adequate model for explosions and atmospheric releases; however, other release modeling programs could be added to provide additional capabilities, including surface spills. 


\section{References}

Bennett, M. 2003. TICs, TIMs and Terrorists. Today's Chemist at Work: 21-25.

Canada, United Kingdom, and United States (CANUKUS). 1994. Memorandum of understanding on chemical and biological defense (CBD). ITF-25.

Center for Counterproliferation Research. 2000. Joint doctorine for operations in nuclear, biological, and chemical (NBC) environments. Joint Publication 3-11. Washington DC: National Defense University.

Channell, M., M. R. Graves, V. F. Medina, A. B. Morrow, D. Brandon, and C. C. Nestler. 2009. Enhanced tools and techniques to support debris management in disaster response missions. ERDC/EL TR-09-12. Vicksburg, MS: U.S. Army Engineer Research and Development Center.

Cornell University Law School (CULS). Undated. Legal Information Institute. Title 42, Chapter 116, Subchapter II. Available on line at http://www.law.cornell.edu/uscode/ html/uscode42/usc_sup_01_42_10_116_20_Il.html.

Cutclasure,H., and C. McClellan. 2009. Joint consequence manager consequence management. TIC/TIM Task Force Programmic Decision Pre-Brief for Doug Bryce, DJPEO for Chem-Bio Defense.

Dortch, M., M. Zakakhani, J. Furey, R. Meyer, S. Fant, J. Gerald, M. Qasim, H. Fredrickson, and P. Honea. 2005. Data gap analysis and database expansion. ERDC/EL TR-05-16. Vicksburg, MS: U.S. Army Engineer Research and Development Center.

Haefner, J. 2004. US Army, Pacific Theater Geospatial Database: A model for synergistic geospatial intelligence. Available on-line: http://gis.esri.com/library/ userconf/proc07/docs/pap2139. Retrived 9/13/2007.

Hauschild, V. D. 2004 . Assessing military exposure during deployment. Military Medicine 169:142.

Hauschild, V. D., and A. P. Lee. 2004. Assessing chemical exposures during military deployments. Military Medicine 169:142-146.

Hill, S. 2009. Army environmental requirements and technology assessments - FYo9. Aberdeen Proving Ground, MD: U.S. Army Environmental Command.

Jederberg, W. W., K. R. Still, and G. B. Briggs. 2002. The utilization of risk assessment in tactical command decisions. The Science of the Total Environment 288:119-129.

Johnson, B. E., V. F. Medina, and D. Cunniff. 2008. Numerical simulation of depleted uranium transport in a desert environmen using a distributed parameter watershed model. Practice Periodical of Hazardous, Toxic, and Radioactive Waste Management 10: 179-189. 
Langenberg, J. P. 2007. Risk management of exposure to chemicals under operational conditions. Paper presented at the HFM-129 Symposium on Defence Aganst the Effects of Chemical Hazards: Toxicology, Diagnosis, and Medical Countermeasures. Edinburg, Scotland.

Mauroni, A. J. 2002. How the chemical corp addresses hazardous materials. CBS Interactive Business Network. Available on-line at: $\underline{\mathrm{http}} / / /$ findarticles.com/p/ articles/mi_molun/is_2002_Feb/ai_84668287/

National Oceanic and Atmospheric Administration (NOAA). undated. CAMEO Chemicals. http://cameochemicals.noaa.gov/.

National Fire Protection Association (NFPA). 2006. NFPA 704 - Standard System for the Identification of the Hazards of Materials for Emergency Response, 2007 Edition.

National Institute for Occupational Safety and Health (NIOSH). Undated. International Chemical Safety Cards (ICSC). http://www.cdc.gov/niosh/ipcs/icstart.html.

National Institute for Occupational Safety and Health (NIOSH). 2005. NIOSH pocket guide to chemical hazards. NIOSH Publication Number 2005-149, http://www.cdc.gov/niosh/npg/.

Resta, J. J. 2001. International Task Force 40: Toxic industrial chemicals (TICs) Operational and medical concerns. US/UK/CA Memorandum of Understanding on the Research, Development, and Acquisition of Chemical, Biological, and Radiological Defense Material. Unclassified Presentation.

Small, L. 2002. Toxic Industrial Chemical: A future weapons of mass destruction threat. MS thesis. Fort Leavenworth, KS: US Army Command and General Staff College.

Steumpfle, A. K., S. J. Armour, C. A. Boulet, and D. J. Howells. 1996. Final report of International Task Force (ITF)-25: Hazard from Industrial Chemicals. US/UK/CA Memorandum of Understanding of Chemical and Biological Defence.

United States, British, Canadian, Australian and New Zealand Armies' Program Office (USBCANZPO). 2009. Identification and management of toxic industrial materials (TIM) and chemical biological radiological and nuclear (CBRN) munitions during coalition operations. International Task Force-40.

U.S. Army Center for Health Promotion and Preventive Medicine (USACHPPM). 2003. Industrial chemical prioritization and determination of critical hazards of concern, technical annex and supporting documents for international task force (ITF)-40, industrial chemical hazards: Medical and operational concerns. Report 47-EM-6154-03.

U.S. Army Engineer Research and Development Center (ERDC), Environmental Laboratory (EL). 2005. Geospatial Risk Assessment Modeling System (GeoRAMS). ERDC/EL TR-05-5. Vicksburg, MS: U.S. Army Engineer Research and Development Center.

U.S. Department of the Army (USDOA). 1994. Intelligence preparation of the battlefield. US Army Field Manual, FM34-130 - IPB. 
U.S. Department of the Army (USDOA). 2004. Operational terms and graphics. Army Field Manual, FM 1-02.

U.S. Department of the Army (USDOA). 2010. Environmental considerations in military operations. Army Field Manual, FM-3-24.5.

U.S. Department of Defense (USDoD). 2001. Department of Defense dictionary of military and associated terms. Joint Publication 1-02.

U.S. Department of Transportation (USDOT). 2008. Emergency response guidebook.

United States Environmental Protection Agency (USEPA). 2009. Senators press DoD IG for investigation of soldiers' exposure. Risk Policy Report. August 25, 2009, p 89. www.InsideEPA.com.

U.S. Environmental Protection Agency (USEPA). 2010. Emergency Planning and Community-Right-to-Know Act. Guidance on reporting options for Sections 311 and 312 and some interpretations. EPA 500-F-10001. Available On-line: http://www.epa.gov/oem/docs/chem/guide_interp_fs.pdf.

Van Antwerp, R. L. 2010. Operations Order 2010-36. Requirements for evaluation, response, and reporting of incidents involving hazardous or toxic agents during overseas contingency operations (OCO). Classification - For Official Use Only. 


\section{Appendix A: Chemical Database for the TIC- Master Program}

To access the chemical database for the TIC-Master Program, contact Dr. Victor Medina at:

Victor.F.Medina@usace.army.mil 


\section{Appendix B: Instructions for Using the EPA TRI Database to Create ICCW}

Instructions to fill crosswalk with data for one industrial six-digit code:

A. Perform custom query of U.S. Environmental Protection Agency (USEPA) Toxic Release Inventory (TRI) database

1. Go to customized TRI query at http://www.epa.gov/enviro/htm//tris/adhoc.html

2. Select Facility information

3. Select Form R Reporting information

4. Select Submission NAICS Codes

5. Click the Step 2 button

6. Select tri_facility

7. Select v_tri_reporting_form

8. Select v_tri_submission_naics

9. Click the Step 3 button

10. In TRI_FACILITY, select TRI Facility ID (and state abbreviation, if desired)

11. In V_TRI_REPORTING_FORM, select Chemical Name, Trichemical ID, and CAS chem name

12. In V_TRI_SUBMISSION_NAICS, select NAICS Codes

13. Click the Step 4 button

14. In the NAICS Code section, enter the NAICS code in the Search Value box, and select TRI CHEM ID

B. Save this file to disc and then open up in EXCEL. Resave the file as "Naics\#\#\#\#\#\#TRIcustom\#.xls" where the first \#s are for the code and the last number is for the number query that has been performed on that code (usually 1).

C. Perform the sequence below to manipulate data in this new EXCEL file for entry into the crosswalk:

1. Copy CAS and chem name to two new columns, then select both columns and sort based on CAS (rename CAS sort) (when selecting the two columns, do not include the mixture numbers and mixture names, only the chemicals with a CAS (these may be used at a later time)). 
2. Select CAS sort, select menu item Data / Pivot table and chart, drop CAS sort into Rows, then into Drop Data Items. This gives a new sheet with all chemical CAS's plus the number of entries for each. Call the new sheet CAS frequency.

3. Do the same thing as step 3 for the first column of facility IDs in the original sheet to get the total number of facilities reporting, name the new sheet Facility Count, type that number in a cell at the top of the sheet for future reference (\# total facilities).

4. On the CAS frequency sheet, copy both columns to new columns, in another column type in a function for the first row to calculate \%probability that a facility will report a certain chemical (e.g., $=100 x \mathrm{x} 2 / \#$ facilities, where \#facilities is the integer value obtained from step 3). Calculate this for the entire chemical list by selecting that formula cell and stretching it down the entire column to include all cells that are in rows with chemicals (release the mouse and it will automatically fill the cells). Name that new row \%prob facility.

5. Now create a matching row of chemical names to correspond to the trimmed-down CAS names. Create a new sheet and call it "Filtered list of names," copy the sorted column of names (next to CAS sort) from the original sheet, and paste it in this new sheet. Select the column and select from the menu Data/Filter/Advanced Filter/and then check the box for unique records only. This will replace the list of names with a filtered list that will list each name only once.

6. Go to the CAS frequency sheet, copy the CAS column and paste the copy into a new column to the right of the \% prob facility column, call it CAS, copy the column created in step 5 to the column to the right of CAS and call it sorted CAS name, copy the \%prob facility column and paste special - value only- to the column next to sorted CAS name and call it \%prob facility value only. Review the three columns to ensure that the number of entries match and that the names and CAS's also match up. Two names could be assigned to the same CAS, which would result in an extra name entry in the sorted names.

7. Now copy the three columns of data to the crosswalk: count the number of rows (chemicals in the CAS sort column), and insert that many rows into the crosswalk after the appropriate code in the two columns titled NAICS_Code and NAICS_Title. Select the number of cells to insert at the point of insertion and choose from menu insert/cells/shift cells down). Paste that code and name to all the cells just created (copy two cells, select all new cells, paste). Back in CAS Frequency, copy the three columns of data (be careful to only select the rows to copy), and paste 
into the crossover next to the newly created cells. Convert the new text to Arial font, and verify that the sheets are cleaned up, saved, and named properly for future reference. 


\title{
Appendix C: Standard Operating Procedures (SOPs) Used in This Study
}

Acronyms and abbreviations used in this appendix

\author{
atm: Atmosphere(s) \\ BCF: Bioconcentration Factor \\ C: Centigrade \\ $\mathrm{cm}$ : centimeter(s) \\ cu: cubic
}

deg: degree(s)

EPA: United States Environmental Protection Agency

EPA RfC: EPA reference concentration

EPA RfD: EPA reference dose

FW: Fresh Water

h: hour

hc: Human Health Carcinogen

IDLH: Immediately Dangerous to Life and Health

kg: Kilogram

Koc: Organic carbon adsorption coefficient

Kow: Octanol Water Coefficient

l: liter 
m: meter

MCL: Maximum Contaminant Level

$\mathrm{m}: \operatorname{meter}(\mathrm{s})$

mg: milligram(s)

mm: millimeter(s)

mol: mole(s)

Pa: Pascal(s)

ppb: parts per billion

ppm: parts per million

PQL: Practical Quantification Limit

PRG: Preliminary Remediation Goal

UTS: Universal Treatment Standards

ug: microgram 


\section{Acrolein 107-02-8}

\section{ENVIRONMENTAL TRANSPORT, DISTRIBUTION, AND TRANSFORMATION}

Air: Acrolein emitted to air reacts primarily with photochemically generated hydroxyl radicals in the troposphere (Ghilarducci \& Tjeerdema, 1995). Minor processes include direct photolysis, reaction with nitrate radicals, and reaction with ozone (Atkinson et al., 1987; Haag et al., 1988a; Howard, 1989; BUA, 1994). Acrolein has been detected in rainwater, indicating that it may be removed by wet deposition (Grosjean \& Wright, 1983). The calculated atmospheric half-life of acrolein, based on rate constants for hydroxyl radical reaction, is between 3.4 and $33.7 \mathrm{~h}$ (Atkinson, 1985; Edney et al., 1986b; Haag et al., 1988a; Howard, 1989; Howard et al., 1991; BUA, 1994). The overall reactivitybased half-life of acrolein in air, as estimated by Mackay et al. (1995), is less than $10 \mathrm{~h}$. Based on these short estimated half-lives, acrolein is not a candidate for long-range atmospheric transport.

Water: Acrolein is removed from surface water primarily by reversible hydration, biodegradation by acclimatized microorganisms, and volatilization (Irwin, 1987; Haag et al., 1988b; Howard, 1989; ATSDR, 1990; Springborn Laboratories, 1993). In groundwater, acrolein is removed by anaerobic biodegradation and hydrolysis (Chou \& Spanggord, 1990a). The overall reactivity-based half-life of acrolein in surface water is estimated to be between 30 and $100 \mathrm{~h}$ (Mackay et al., 1995). In groundwater, half-lives of 11 days and 336-1344 h (14-56 days) are estimated based on aerobic and anaerobic degradation, respectively (Howard et al., 1991). Observed dissipation half-lives of acrolein applied as a herbicide in irrigation range from 7.3 to $10.2 \mathrm{~h}$ (Jacobson \& Gresham, 1991a, b, c; Nordone et al., 1996a). The relatively short observed half-lives of acrolein in surface waters make long-range aquatic transport unlikely. However, groundwater contamination could result contaminant exposures.

Sediment: In sediment/water systems, acrolein undergoes hydrolysis, self-oxidation, and biodegradation. Experimental half-lives of $7.6 \mathrm{~h}$ and 10 days were determined for aerobic and anaerobic conditions, respectively (Smith et al., 1995). An overall reactivity-based half-life is estimated by Mackay et al. (1995) to be between 100 and $300 \mathrm{~h}$. Because of its low organic carbon/water partition coefficient (Koc) and high water solubility, acrolein is not expected to significantly adsorb to suspended solids or sediments, nor are these suspended solids or sediments expected to significantly absorb acrolein from water (Irwin, 1988; Howard, 1989). 
Soil: In the terrestrial environment, acrolein undergoes biodegradation, hydrolysis, volatilization, and irreversible sorption to soil (Irwin, 1988; Howard, 1989; Chou \& Spanggord, 1990b). These processes are expected to significantly decrease the high infiltration rate of acrolein estimated from its low experimental Koc (Irwin, 1988). The overall reactivity-based half-life of acrolein in soil is estimated to be between 30 and 100 h (Mackay et al., 1995).

Biota: Based on the high water solubility, low octanol/ water partition coefficient (Kow), and high reactivity of acrolein, uptake by organisms is predicted to be low. A bioconcentration factor (BCF) of 344 and a half-life of greater than 7 days were reported for acrolein in bluegill (Lepomis macrochirus) following exposure to acrolein at a mean concentration of $13 \mu \mathrm{g} / \mathrm{l}$ for a 28 -day period (Barrows et al., 1980). However, these values may be overestimates, as the total $14 \mathrm{C}$ measured in the fish may have included metabolites. A lower BCF of 0.6 was estimated using the linear regression equation of Veith et al. (1980) and a log Kow of 0.01 for acrolein. Acrolein was not detected in the tissues of fish and shellfish sampled 1 day after a second exposure to [14C]acrolein in water (0.02 and $0.1 \mathrm{mg} /$ litre for the first and second exposures, respectively) over a 1week period. The presence of metabolites indicates that these species were able to rapidly metabolize acrolein and its residues (Nordone et al., 1998). Based on these results and the low reported BCFs, acrolein is unlikely to bioaccumulate or bioconcentrate significantly in aquatic organisms (Howard, 1989; ATSDR, 1990; DFO, 1995; Nordone et al., 1996b). Absorption of acrolein by terrestrial plants is poor (WSSA, 1983).

Environmental partitioning : Fugacity modeling was conducted to characterize key reaction, intercompartment, and advection (movement out of a system) pathways for acrolein and its overall distribution in the environment. A steady-state, non-equilibrium model (Level III fugacity model) was run using the methods developed by Mackay (1991) and Mackay \& Paterson (1991). Assumptions, input parameters, and results are presented in Mackay et al. (1995) and summarized here. Values for input parameters were as follows: molecular mass, 56.06 ; melting point, $186.95{ }^{\circ} \mathrm{C}$; water solubility, $208 \mathrm{~g} / \mathrm{l}$; vapour pressure, $36.5 \mathrm{kPa}$ at $20{ }^{\circ} \mathrm{C}$; $\log \mathrm{Kow}, 0.01$; Henry's law constant, $9.8 \mathrm{~Pa} @ \mathrm{~m} 3 / \mathrm{mol}$; halflife in air, $5 \mathrm{~h}$; half-life in water, $55 \mathrm{~h}$; half-life in soil, $55 \mathrm{~h}$; half-life in sediments, $170 \mathrm{~h}$. Modeling was based on an assumed default emission rate of $1000 \mathrm{~kg} / \mathrm{h}$ into a region of $100000 \mathrm{~km} 2$, which includes a surface water area (20 m deep) of $10000 \mathrm{~km} 2$. The height of the atmosphere was set at $1000 \mathrm{~m}$. Sediments and soils were assumed to have an organic carbon content of $4 \%$ and $2 \%$ and a depth of $1 \mathrm{~cm}$ and $10 \mathrm{~cm}$, respectively. The estimated percent distribution predicted by this model is not affected by the assumed emission rate. Results of the modeling indicate that acrolein behaves differently depending on the medium to which it is released. Generally, when acrolein is continuously discharged into a specific medium, most of it can be expected to remain in 
that medium. For example, if discharged into air, almost all of it will exist in the atmosphere, with very small amounts in soil and water. The same applies for discharge to water and soil (Mackay et al., 1995). These predicted distributions suggest that acrolein does not tend to partition from one compartment to another. It could also be possible that when acrolein does partition to another compartment, its persistence in that second compartment is so short that little remains there.

\section{Regulatory}

MCL (Drinking Water): None

Universal Treatment Standards (UTS): $0.29 \mathrm{mg} / \mathrm{l} 2$ in wastewater standards

Controlling Water Quality Criterion for Surface Water:

Basis: Aquatic Toxicity Criterion Concentration: $21 \mathrm{ug} / \mathrm{L}$ Criterion Quantitation Limit: 5 ug/L

EPA Rf D: $0.0005 \mathrm{mg} / \mathrm{kg}$-day

EPA RfC: $0.000002 \mathrm{mg} / \mathrm{m} 3$

PRG: residential soil mg/kg industrial soil mg/kg ambient air ug/m tap water $\mathrm{ug} / \mathrm{L}$
0.1
0.345
0.21
0.042

Soil PQL: $0.5 \mathrm{mg} / \mathrm{kg}$

\section{Remediation}

Due to its high reactivity, acrolein does not tend to persist in the environment, and its intercompartmental movement is small. Therefore, there does not appear to be much information on its treatment. For short-term spills, adsorbent could be an effective approach. If soil or groundwater should occur, and clean-up is deemed necessary, acrolein does appear to be susceptible to bioremediation.

\section{References}

Atkinson R (1985) Kinetics and mechanisms of the gas-phase reactions of hydroxyl radicals with organic compounds under atmospheric conditions. Chemical Reviews, 85:69-201. 
Atkinson R, Aschmann SM, Goodman MA (1987) Kinetics of gasphase reactions of nitrate radicals with a series of alkynes, haloalkenes, and alpha, beta-unsaturated aldehydes. International Journal of Chemical Kinetics, 19:299-308.

ATSDR (1990) Toxicological profile for acrolein. Atlanta, GA, US Department of Health and Human Services, Public Health Service, Agency for Toxic Substances and Disease Registry, 145 pp. (ATSDR/TP-90/o1).

BUA (1994) Acrolein. GDCh Advisory Committee on Existing Chemicals of Environmental Relevance. Stuttgart, S. Hirzel Publishers, 236 pp. (BUA Report No. 157).

DFO (1995) Discussion paper for Magnacide-H® (acrolein). Ottawa, Ontario, Department of Fisheries and Oceans.

Ghilarducci DP, Tjeerdema RS (1995) Fate and effects of acrolein. Reviews of Environmental Contamination and Toxicology, 144:95-146.

Chou T-W, Spanggord RJ (1990a) Estimation of the anaerobic biotransformation rates for acrolein (Magnacide $囚$ H Herbicide, Magnacide $₫$ B Biocide) in soil-water mixtures. Prepared by SRI

International for Baker Performance Chemicals, Inc., Houston, TX, 414 pp.

Chou T-W, Spanggord RJ (1990b) Estimation of the aerobic biotransformation rates for acrolein (Magnacide® Herbicide, Magnacide $₫$ Biocide) in soil. Prepared by SRI International for Baker Performance Chemicals, Inc., Houston, TX, 414 pp.

Grosjean D, Wright B (1983) Carbonyls in urban fog, ice fog, cloudwater and rainwater. Atmospheric Environment, 17:2093-2096.

Edney EO, Kleindienst TE, Corse EW (1986b) Room temperature rate constants for the reaction of $\mathrm{OH}$ with selected chlorinated and oxygenated hydrocarbons. International Journal of Chemical Kinetics, 18:1355-1371.

Haag WR, Yao CD, Pettit T, Mill T (1988b) Estimation of hydrolysis rate constants for acrolein (Magnacide $\AA$ H Herbicide, Magnacide $\AA$ B Microbiocide) in the environment. Prepared by SRI International for Baker Performance Chemicals, Inc., Houston, TX, 54 pp. 
Howard P (1989) Handbook of environmental fate and exposure data for organic chemicals. Volume 1. Large production and priority pollutants. Boca Raton, FL, Lewis Publishers, $12 \mathrm{pp}$.

Howard P, Boethling R, Jarvis W, Meylan W, Michalenko E (1991) Handbook of environmental degradation rates. Boca Raton, FL, Lewis Publishers.

Irwin K (1987) Henry's law constant for acrolein (Magnacide ${ }^{\circledR}$ H Herbicide, Magnacide $₫$ B Microbiocide). Prepared by SRI International for Baker Performance Chemicals, Inc., Houston, TX.

Irwin K (1988) Soil adsorption coefficient for acrolein (Magnacide $囚$ H Herbicide and Magnacide $®$ B Microbiocide). Prepared by SRI International for Baker Performance Chemicals, Inc., Houston, TX, 24 pp.

Jacobson B, Gresham M (1991a) Magnitude of residue for acrolein in potable water Arizona site. Prepared by Analytical Bio-Chemistry Laboratories, Inc., for Baker Performance Chemicals Inc., Houston, TX, 178 pp.

Jacobson B, Gresham M (1991b) Magnitude of residue for acrolein in potable water Washington site. Prepared by Analytical Bio-Chemistry Laboratories, Inc., for Baker Performance Chemicals Inc., Houston, TX, 230 pp.

Jacobson B, Gresham M (1991c) Aquatic field dissipation for acrolein. Prepared by Analytical Bio-Chemistry Laboratories, Inc., for Baker Performance Chemicals Inc., Houston, TX, 244 pp.

Mackay D, Shiu WY, Ma KC (1995) Illustrated handbook of physical-chemical properties and environmental fate for organic chemicals. Volume IV. Boca Raton, FL, Lewis Publishers.

Mackay D (1991) Multimedia environmental models: the fugacity approach. Chelsea, MI, Lewis Publishers.

Mackay D, Paterson S (1991) Evaluating the multimedia fate of organic chemicals: a Level III fugacity model. Environmental Science and Technology, 25:427.

Mackay D, Shiu WY, Ma KC (1995) Illustrated handbook of physical-chemical properties and environmental fate for organic chemicals. Volume IV. Boca Raton, FL, Lewis Publishers. 
Nordone AJ, Matherly R, Bonnivier B, Doane R, Caravello H, Paakonen S, Parent RA (1996a) The mobility and degradation of acrolein in agricultural canals treated with Magnacide H Herbicide. Chemosphere, 32:807-814.

Nordone AJ, Dotson TA, Kovacs MF, Doane R, Biever RC (1998) The metabolism of [14C] acrolein (Magnacide $\mathrm{H} \circledast$ Herbicide): nature and magnitude of residues in freshwater fish and shellfish. Environmental Toxicology and Chemistry, 17:276-281.

Nordone AJ, Dotson TA, Kovacs MF, Doane R, Biever RC (1996b) The metabolism of [14C] acrolein (Magnacide $\mathrm{H} 囚$ Herbicide): nature and magnitude of residues in freshwater fish and shellfish. In: Proceedings of the 17th Annual SETAC Meeting, 16-21 November, Society of Environmental Toxicology and Chemistry, Washington, DC.

Smith AM, Mao J, Doane RA, Kovacs MF Jr (1995) Metabolic fate of (14C) acrolein under aerobic and anaerobic aquatic conditions. Journal of Agricultural and Food Chemistry, 43:2497-2503.

Springborn Laboratories (1993) (14C-Acrolein) - Determination of the aerobic aquatic metabolism. Prepared for Baker Performance Chemicals Inc., Houston, TX, 125 pp. (SLI No. 91-3-3747).

Veith GD, Macek KJ, Petrocelli SR, Carroll J (1980) An evaluation of using partition coefficients and water solubility to estimate bioconcentration factors for organic chemicals in fish. In: Eaton JG, Parrish PR, Hendricks AC, eds. Aquatic toxicology. Philadelphia, PA, American Society for Testing and Materials, pp. 116-129 (ASTM STP 707). 


\section{Acrylonitrile $\quad \mathbf{1 0 7 - 1 3 - 1}$}

\section{ENVIRONMENTAL TRANSPORT, DISTRIBUTION, AND TRANSFORMATION}

Air: Acrylonitrile emitted to air reacts primarily with photochemically generated hydroxyl radicals in the troposphere (Atkinson et al., 1982; Edney et al., 1982; Munshi et al., 1989; US DHHS, 1990; Bunce, 1996). The atmospheric half-life, based on hydroxyl radical reaction rate constants, is calculated to be between 4 and 189 hours (h) (Callahan et al., 1979; Cupitt, 1980; Edney et al., 1982; Howard, 1989; Grosjean, 199ob; Kelly et al., 1994). Modeling of environmental partitioning is based on a mean half-life for acrylonitrile in air of $55 \mathrm{~h}$. The reaction of acrylonitrile with ozone and nitrate is slow, because of the absence of chlorine and bromine atoms in the molecule, and is not likely to constitute a major route of degradation (Bunce, 1996). The reaction of hydroxyl radicals with acrylonitrile yields formaldehyde and, to a lesser extent, formic acid, formyl cyanide, carbon monoxide, and hydrogen cyanide (Edney et al., 1982; Spicer et al., 1985; Munshi et al., 1989; Grosjean, 1990a).

Water: Acrylonitrile in water can be biodegraded by acclimatized microorganisms or volatilized (Going et al., 1979). In water, half-lives of 30-552 h are estimated based on aqueous aerobic biodegradation (Ludzack et al., 1961; Going et al., 1979; Howard et al., 1991). Modeling of environmental partitioning based on a mean half-life for acrylonitrile in water of $170 \mathrm{~h}$ (7 days). The half-life based on volatilization is 1-6 days (Howard et al., 1991). The hydrolysis of acrylonitrile is slow, with half-lives under acidic and basic conditions of 13 and 188 years, respectively (Ellington et al., 1987). Acrylonitrile has an initial inhibitory effect on activated sludge systems and other microbial populations and does not meet the criteria of Organization for Economic Co-operation and Development (OECD) Test Method 301C for ready biodegradability (Chemicals Inspection and Testing Institute of Japan, 1992; AN Group, 1996; BASF AG, 1996). However, acrylonitrile will be extensively degraded (95-100\%) following a short acclimation period if emitted to wastewater treatment plants (Tabak et al., 1980; Kincannon et al., 1983; Freeman \& Schroy, 1984; Watson, 1993).

Soil and sediment: Acrylonitrile is biodegraded in a variety of surface soils (Donberg et al., 1992) and by isolated strains of soil bacteria and fungi (Wenzhong et al., 1991). Concentrations of acrylonitrile up to $100 \mathrm{mg} / \mathrm{kg}$ were degraded in under 2 days (Donberg et al., 1992). Similar breakdown by microbial populations present in sediment is likely (DMER \& AEL, 1996; EC, 2000). Results of experimental studies (Zhang et al., 1990) or soil sorption coefficients calculated by quantitative structure-activity relationships (Koch \& Nagel, 1988; Walton et al., 1992) or based on water solubility (Kenaga, 1980) indicate 
little potential for adsorption of acrylonitrile to soil or sediments. A half-life of acrylonitrile in soil of 6-7 days has been reported (Howard et al., 1991; Donberg et al., 1992). Based on biodegradability and the soil partition coefficient, the half-life of acrylonitrile in soil was classified in the category of 300 days (EC, 2000). Modeling of environmental partitioning is based on a mean half-life for acrylonitrile in soil of $170 \mathrm{~h}$ (7 days). The half-life in the oxic zone of sediment can be assumed to be similar.

Biota: Bioaccumulation of acrylonitrile is not anticipated, given experimentally derived values of the octanol/water partition coefficient (log Kow) ranging from 0.92 to 1.2 (mean o.25) (Tanii \& Hashimoto, 1984; Sangster, 1989) and a log bioconcentration factor (log $\mathrm{BCF}$ ) of o calculated from the water solubility of acrylonitrile (EC, 2000). $\log \mathrm{BCF}$ values were 0.48-1.68 in bluegill (Lepomis macrochirus) (Barrows et al., 1980) and rainbow trout (Oncorhynchus mykiss) (Lech et al., 1995). The experimentally derived log BCF of 1.68 reported by Barrows et al. (1980) in whole-body tissue of bluegill may be an overestimate, due to uptake of $14 \mathrm{C}$-labelled degradation products in addition to acrylonitrile and to cyanoethylation of macromolecules (EC, 2000).

Environmental partitioning: Fugacity modeling was conducted to characterize key reaction, intercompartment, and advection (movement out of a compartment) pathways for acrylonitrile and its overall distribution in the environment. A steady state, nonequilibrium model (Level III fugacity model) was run using the methods developed by Mackay (1991) and Mackay \& Paterson (1991). Values for input parameters were as follows: molecular mass, $53.06 \mathrm{~g} / \mathrm{mol}$; water solubility, $75.5 \mathrm{~g} / \mathrm{litre}$; vapour pressure, 11.0 $\mathrm{kPa}$; log Kow, 0.25; Henry's law constant, $11 \mathrm{~Pa} @ \mathrm{~m} 3 / \mathrm{mol}$; half-life in air, 55 h; half-life in water, $170 \mathrm{~h}$; half-life in soil, $17 \mathrm{O} \mathrm{h}$; half life in sediments, $550 \mathrm{~h}$. Modeling was based on an assumed default emission rate of $1000 \mathrm{~kg} / \mathrm{h}$ into a region of $100000 \mathrm{~km}^{2}$, which includes a surface water area (20 m deep) of $10000 \mathrm{~km}^{2}$. The height of the atmosphere was set at $1000 \mathrm{~m}$. Sediments and soils were assumed to have an organic carbon content of $4 \%$ and $2 \%$ and a depth of $1 \mathrm{~cm}$ and $10 \mathrm{~cm}$, respectively. The estimated percent distribution predicted by this model is not affected by the assumed emission rate. Modeling indicates that when acrylonitrile is continuously discharged into a specific medium, most of it (84-97\%) can be expected to be present in that medium (DMER \& AEL, 1996). More specifically, Level III fugacity modeling by DMER \& AEL (1996) predicts that: when released into air, the distribution of mass is $92.8 \%$ in air, $6.4 \%$ in water, $0.8 \%$ in soil, and $0.0 \%$ in sediment; when released into water, the distribution of mass is $2.5 \%$ in air, $97.3 \%$ in water, $0.0 \%$ in soil, and $0.1 \%$ in sediment; and when released into soil, the distribution of mass is $4.4 \%$ in air, $11.9 \%$ in water, $83.7 \%$ in soil, and $0.0 \%$ in sediment. The major removal mechanisms in air, water, and soil are reaction within the medium and, to a lesser degree, advection and volatilization. Abiotic and biotic degradation in various compartments result in low persistence overall and little if any 
bioaccumulation. Owing to the paucity of data on concentrations of acrylonitrile in environmental media, fugacity modeling with version 4 of the ChemCAN3 model (Mackay et al., 1995) was also conducted with the conservative assumption that all known releases in 1996 (Environment Canada, 1997) in Canada occurred in southern Ontario. Release to air was considered to be approximately 19 tonnes per year, with simultaneous release to water of 0.53 tonnes per year. Since the half-life of acrylonitrile in air is the major determinant of its fate in the environment, the model was run using the minimum, median, and maximum half-life values $(4,55$, and $189 \mathrm{~h})$ under summer, winter, and year-round conditions. Modeling predicted distribution primarily to air (41.9-78.1\%) and water $(21.6-57.9 \%)$.

\section{Regulatory}

MCL (Drinking Water): None

UTS: $0.24 \mathrm{mg} / \mathrm{L}^{2}$ in wastewater standards and $84 \mathrm{mg} / \mathrm{kg}^{3}$ non wastewater standards

Soil PQL: $0.5 \mathrm{mg} / \mathrm{kg}$

EPA Rf D: $0.001 \mathrm{mg} / \mathrm{kg} / \mathrm{d}$ provisional

EPA Rf C: $2 \mu \mathrm{g} / \mathrm{m} 3$

PRG: residential soil mg/kg industrial soil mg/kg ambient air ug/m3 tap water ug/L $\begin{array}{llll}0.21 & 0.51 & 0.028 & 0.039\end{array}$

Fresh Water (FW) Criteria: Human Health: 0.051 ug/L (hc) Saline Water Criteria: 0.25 ug/L (hc) 'Human health carcinogen'

\section{Remediation}

Land spill: Dig a pit, pond, lagoon, or holding area to contain liquid or solid material. /SRP: If time permits, pits, ponds, lagoons, soak holes, or holding areas should be sealed with an impermeable flexible membrane liner. Dike surface flow using soil, sand bags, foamed polyurethane, or foamed concrete. Absorb bulk liquid with fly ash, cement powder, sawdust, or commercial sorbents. Apply "universal" gelling agent to immobilize spill.

Air spill: Apply water spray or mist to knock down vapors. Combustion products include corrosive or toxic vapors.

Water spill: Use natural barriers or oil spill control booms to limit spill travel. Use surface 
active agent (eg, detergent, soaps, or alcohols) if approved by EPA. Inject "universal" gelling agent to solidify encircled spill and increase effectiveness of booms. Add calcium hypochlorite. If dissolved, in region of $10 \mathrm{ppm}$ or greater concentration, apply activated carbon at ten times the spilled amount. Use mechanical dredges or lifts to remove immobilized masses of pollutants and precipitates (Association of American Railroads).

\section{References}

Atkinson R, Baulch DL, Cox RA (1992) Evaluated kinetic and photochemical data for atmospheric chemistry. Supplement IV. IUPAC Subcommittee on Gas Kinetic Data Evaluation for Atmospheric Chemistry. Journal of physical and chemical reference data, 21(6):1125-1568.

Barrows ME, Petrocelli SR, Macek KJ (1980) Bioconcentration and elimination of selected water pollutants by bluegill sunfish (Lepomis macrochirus). In: Haque R, ed. Dynamics, exposure, and hazard assessment of toxic chemicals. Ann Arbor, MI, Ann Arbor Science Publishers Inc., pp. 379-392.

Bunce NJ (1996) Atmospheric properties of substances on the Priority Substances List \#2 (PSL2). Report to Environment Canada. Guelph, Ontario, University of Guelph.

Callahan MA, Slimak MW, Gabel NW, May IP, Fowler CF, Freed JR, Jennings P, Durfee RL, Whitmore FC, Maestri B, Mabey WR, Holt BR, Gould C (1979) Water related environmental fate of 129 priority pollutants. Springfield, VA, Versar, Inc. (EPA-440-479-029a,b) [cited in Mackay et al., 1995].

Cupitt LT (1980) Fate of toxic and hazardous materials in the air environment. Research Triangle Park, NC, US Environmental Protection Agency, Office of Research and Development, Environmental Services Research Laboratory, 28 pp. (EPA-60o/3-80o84).

DMER, AEL (1996) Pathways analysis using fugacity modelling of acrylonitrile for the second Priority Substances List. Report prepared for Chemicals Evaluation Division, Commercial Chemicals Evaluation Branch, Environment Canada, by Don Mackay Environmental Research, Peterborough, Ontario, and Angus Environmental Limited, Don Mills, Ontario.

Donberg PA, Odelson DA, Klecka GM, Markham DA (1992) Biodegradation of acrylonitrile in soil. Environmental toxicology and chemistry, 11:1583-1594. 
DMER, AEL (1996) Pathways analysis using fugacity modelling of acrylonitrile for the second Priority Substances List. Report prepared for Chemicals Evaluation Division, Commercial Chemicals Evaluation Branch, Environment Canada, by Don Mackay Environmental Research, Peterborough, Ontario, and Angus Environmental Limited, Don Mills, Ontario.

EC (2000) Risk assessment of acrylonitrile CAS No. 107-13-1 EINECS No. 203-466-5. Final draft March 2000, prepared by the Hazardous Substances Assessment Unit of the Health and Safety Authority, Dublin, for the European Community.

Edney E, Mitchell S, Bufalini JJ (1982) Atmospheric chemistry of several toxic compounds. Washington, DC, US Environmental Protection Agency, Environmental Services Research Laboratory,120 pp. (EPA-600/3-82-092).

Ellington JJ, Stancil FE, Payne WD (1987) Measurement of hydrolysis rate constants for evaluation of hazardous waste land disposal. Vol. 1. Data on 32 chemicals. Washington, DC, US Environmental Protection Agency (EPA-60o/3-86-043; NTIS PB87-140 349/GAR) [cited in Mackay et al., 1995].

Environment Canada (1989b) Atlantic Region Federal-Provincial Toxic Chemical Survey of Municipal Drinking Water Sources. Data summary report. Province of New Brunswick. 1985-1988. Moncton, New Brunswick, Environment Canada, Water Quality Branch (IWD-AR-WQB-89-155).

Freeman RA, Schroy JM (1984) Air stripping of acrylonitrile from waste-treatment plants. Environmental progress, 3:26-33.

Going J, Kuykendaho P, Long S, Onstot J, Thomas K (1979) Environmental monitoring near industrial sites: Acrylonitrile. Washington, DC, US Environmental Protection Agency (EPA-560/6-79-003).

Grosjean D (1990a) Atmospheric chemistry of toxic contaminants. 3. Unsaturated aliphatics: acrolein, acrylonitrile, maleic anhydride. Journal of the Air and Waste Management Association, 40(12):1664-1669.

Grosjean D (1990b) Atmospheric chemistry of toxic contaminants. 1. Reaction rates and atmospheric persistence. Journal of the Air and Waste Management Association, 40(10):1397-1402. 
Howard PH (1989) Handbook of environmental fate and exposure data for organic chemicals. Vol. 1. Large production and priority pollutants. Chelsea, MI, Lewis Publishers.

Howard PH, Boethling RS, Jarvis WF, Meylan WM, Michalenko EM (1991) Handbook of environmental degradation rates. Chelsea, MI, Lewis Publishers.

Kelly TJ, Sticksel PR, Pollack AJ, Ramamurthi M, Rench JD (1994) Pollutant monitoring and health risk assessment in Allen County - Lima, Ohio. Presented at the 85th Annual Meeting and Exhibition of the Air and Waste Management Association, Kansas City, MO, 21-26 June 1992, 15 pp.

Kincannon DF, Stover EL, Nichols V, Medley D (1983) Removal mechanisms for toxic priority pollutants. Journal of the Water Pollution Control Federation, 55(2):157-163

Lech JJ, Waddell WJ, Friedman MA, Johnson LR (1995) Uptake, disposition and persistence of acrylonitrile in rainbow trout. Fundamental and applied toxicology, 27:291-294.

Ludzack FJ, Schaffer RB, Bloomhuff RN (1961) Experimental treatment of organic cyanides by conventional processes. Journal of the Water Pollution Control Federation, 33:492-505.

Mackay D (1991) Multimedia environmental models: the fugacity approach. Chelsea, MI, Lewis Publishers.

Mackay D, Paterson S (1991) Evaluating the multimedia fate of organic chemicals: a Level III fugacity model. Environmental science and technology, 25:427-536.

Munshi HB, Rama Rao KVS, Iyer RM (1989) Characterization of products of ozonolysis of acrylonitrile in liquid phase. Atmospheric environment, 23(9):1945-1948.

Spicer CW, Riggin RM, Holden MW, DeRoos FL, Lee RN (1985) Atmospheric reaction products from hazardous air pollutant degradation. Prepared by Battelle-Columbus Laboratories for US Environmental Protection Agency, 88 pp. (PB85-185841).

Tabak HH, Quave SA, Mashni CI, Barth EF (1980) Biodegradability studies for predicting the environmental fate of organic priority pollutants. Cincinnati, OH, US Environmental Protection Agency, Wastewater Research Division, Office of Research and Development, Municipal Environmental Research Laboratory, 327 pp. 
Tanii H, Hashimoto K (1984) Studies on the mechanism of acute toxicity of nitriles in mice. Archives of toxicology, 55:47-54

Sangster J (1989) Octanol-water partition coefficients of simple organic compounds. Journal of physical and chemical reference data, 18:1111-1121, 1227-1229.

US DHHS (1990) Toxicological profile for acrylonitrile. Prepared by Life Systems Inc. for the Agency for Toxic Substances and Disease Registry, Public Health Service, US Department of Health and Human Services, Atlanta, GA, 129 pp. (Report TP-90-02).

Watson HM (1993) A comparison of the effects of two methods of acclimation on aerobic biodegradability. Environmental toxicology and chemistry, 12:2023-2030.

Walton BT, Hendricks MS, Anderson TA, Griest WH, Merriweather R, Beauchamp JJ, Francis CW (1992) Soil sorption of volatile and semivolatile organic compounds in a mixture. Journal of environmental quality, 21:552-558. 


\section{Allyl Alcohol 107-18-6}

\section{ENVIRONMENTAL TRANSPORT, DISTRIBUTION, AND TRANSFORMATION}

Soil: An estimated Koc value of 1.3, determined from a structure estimation method (Meylan et al. 1992), indicates that allyl alcohol is expected to have very high mobility in soil (Swann et al. 1983). Leaching of allyl alcohol from soil surface to deeper layers was found to rank in order of sand $>$ sandy loam $>$ humus sand (Scheunert et al. 1981). The percent leached after 2 days and $400 \mathrm{ml}$ of water with sand containing $0.51 \%$ and $2.89 \%$ organic matter were 100 and 83.3\%, respectively (Scheunert et al. 1981). Freundlich adsorption coefficients for allyl alcohol in Texas soil ( $\mathrm{pH} 7.8,3.25 \%$ organic matter) and Mississippi soil ( $\mathrm{pH} 4.8,<1 \%$ organic matter) were $4.5 \mathrm{X}^{-3} \mathrm{O}^{-3}$ and $3.3 \mathrm{X}^{-4} \mathrm{O}^{-4}$, respectively (Loehr 1989). Volatilization of allyl alcohol from moist soil surfaces is expected to be an important fate process given a Henry's Law constant of 4.99X10-6 atm-cu m/mole (Scheunert et al. 1981). The potential for volatilization of allyl alcohol from dry soil surfaces may exist based upon a vapor pressure of $26.1 \mathrm{~mm} \mathrm{Hg}$ (Daubert and Danner 1989). Half-lives of 10.2 and 9.5 days at $20 \mathrm{deg} C$ were found for allyl alcohol with Texas soil ( $\mathrm{pH} 7.8,3.25 \%$ organic matter) and Mississippi soil ( $\mathrm{pH} 4.8,<1 \%$ organic matter), respectively (Loehr 1989).

Water: An estimated Koc value of 1.3, determined from a structure estimation method (Meylan et al 1992), indicates that allyl alcohol is not expected to adsorb to suspended solids and sediment (Swann et al. 1983). Volatilization from water surfaces is expected (Lyman et al.1990) based upon a Henry's Law constant of 4.99X10-6 atm-cu m/mole (Hine and Mookerjee 1975). Using this Henry's Law constant and an estimation method (Lyman et al.1990), volatilization half-lives for a model river and model lake are 5.7 and 44 days, respectively. According to a classification scheme (Franke et al.1994), an estimated BCF of 3.1, from its log Kow of 0.17 (Hansch et al 1995) and a regressionderived equation (Meylan et al. 1999), suggests the potential for bioconcentration in aquatic organisms is low. Allyl alcohol is not expected to undergo hydrolysis in the environment due to the lack of hydrolyzable functional groups (Lyman et al.19903). In a biodegradation study, allyl alcohol (25 ppm) was found to degrade 100 and $60 \%$ in marine and river water after 3 days at 30 deg C, respectively (Kondo et al. 1988).

Air: According to a model of gas/particle partitioning of semivolatile organic compounds in the atmosphere (Bidleman 1988), allyl alcohol, which has a vapor pressure of $26.1 \mathrm{~mm}$ $\mathrm{Hg}$ at 25 deg C (Daubert and Danner 1989), is expected to exist solely as a vapor in the ambient atmosphere. Vapor-phase allyl alcohol is degraded in the atmosphere by reaction with photochemically-produced hydroxyl radicals; the half-life for this reaction in air is 
estimated to be 13 hours, calculated from its rate constant of $3.0 \mathrm{X}_{10}^{-11} \mathrm{cu} \mathrm{cm} / \mathrm{molecule-}$ sec at $25 \mathrm{deg} C$ that was derived using a structure estimation method (Meylan and Howard 1993). The rate constant for the vapor-phase reaction of allyl alcohol with ozone has been reported as 14.4X10-18 $\mathrm{cu} \mathrm{cm} /$ molecule-sec at 12-22 deg C (Grosjean and Grosjean 1994). This corresponds to an atmospheric half-life of about 0.8 days, at an atmospheric concentration of $7 \mathrm{X}^{10^{11}}$ ozone molecules per $\mathrm{cu} \mathrm{cm}$ (Atkinson and Carter 1984). While allyl alcohol in water was found to degrade to carbon dioxide upon exposure to UV light from a high pressure mercury-vapor lamp through a quartz tube (Knoevenagel and Himmelreich 1976); no data were found regarding the direct photolysis of allyl alcohol by sunlight in air.

Biota: An estimated BCF of 3.1 was calculated for allyl alcohol (SRC), using a log Kow of o.17 (Hansch et al. 1995) and a regression-derived equation (Meylan et al. 1999). According to a classification scheme (Franke et al. 1994), this BCF suggests the potential for bioconcentration in aquatic organisms is low (SRC). The uptake of $14 \mathrm{C}-$ allyl alcohol residues by lettuce and carrots was investigated in the greenhouse (Scheunert et al. 1981). Uptake of residues was higher by carrots than by lettuce, and higher by lettuce roots than by lettuce tops (Scheunert et al.1981). No bioaccumulation was observed.

Environmental Partioning: Using a structure estimation method based on molecular connectivity indices (Meylan et al.1992), the Koc for allyl alcohol can be estimated to be 1.3 (SRC). This estimated Koc value suggests that allyl alcohol is expected to have very high mobility in soi (Swann et al. 1983). Leaching of allyl alcohol from soil surface to deeper layers was found to rank in order of sand $>$ sandy loam $>$ humus sand (Scheunert et al. 1981). The percent leached after 2 days and $400 \mathrm{ml}$ of water with sand containing $0.51 \%$ and $2.89 \%$ organic matter were 100 and $83.3 \%$, respectively (3). Freundlich adsorption coefficients for allyl alcohol in Texas soil ( $\mathrm{pH} 7.8,3.25 \%$ organic matter) and Mississippi soil ( $\mathrm{pH} 4.8,<1 \%$ organic matter) were $4.5 \mathrm{X}_{10}-3$ and $3.3 \mathrm{X}^{-4} \mathrm{O}^{-4}$ respectively (Loehr 1989).

Allyl alcohol is not persistent in the environment. It oxidizes at a moderate rate in the atmosphere and there is also some potential for photolytic degradation. Allyl alcohol is expected to biodegrade under aerobic an anaerobic conditions. It is not susceptible to hydrolysis. Fugacity modeling indicates that over 95\% of allyl alcohol in the environment partitions to water. Very little allyl alcohol will adhere to soil or sediment and it is not expected to bioaccumulate in food chains. 


\section{Regulatory}

MCL (Drinking Water): None

Universal Treatment Standards (UTS): None

EPA Rf D: $0.0005 \mathrm{mg} / \mathrm{kg}$-day

EPA RfC: $0.001 \mathrm{mg} / \mathrm{m}^{3}$

PRG: residential soil mg/kg industrial soil mg/kg ambient air ug/m3 tap water ug/L 310 4400

18 180

\section{Remediation}

Land spill: Dig a pit, pond, lagoon, or holding area to contain liquid or solid material. /SRP: If time permits, pits, ponds, lagoons, soak holes, or holding areas should be sealed with an impermeable flexible membrane liner./ Dike surface flow using soil, sand bags, foamed polyurethane, or foamed concrete. Absorb bulk liquids with fly ash, cement powder, sawdust, or commercial sorbents. Apply "universal" gelling agent to immobilize spill.

Water spill: If dissolved, in region of $10 \mathrm{ppm}$ or greater concentration apply activated carbon at ten times the spilled amount. Use mechanical dredges or lifts to remove immobilized masses of pollutants and precipitates.

Air spill: Apply water spray or mist to knock down vapors (Association of American Railroads) cited in HSDB.

\section{References}

Association of American Railroads/Bureau of Explosives (2002). .Emergency Handling of Hazardous Materials in Surface Transportation.. Pueblo, CO., p. 42.

Atkinson R and Carter WPL. (1984). Kinetics and mechanisms of the gas-phase reaction of ozone with organic compounds under atmospheric conditions. Chem Rev 84:437-70.

Bidleman TF (1988). High volume collection of organic vapors using solid adsorbents. Environ Sci Technol 22: 361-367. 
Daubert TE, Danner RP (1989) Physical and Thermodynamic Properties of Pure Chemicals: Data Compilation. Design Institute for Physical Property Data, American Institute of Chemical Engineers. NY, NY: Hemisphere Pub. Corp. vol 2.

Franke, C., G. Studinger, G. Berger, S. Böhling, U. Bruckmann, D. Cohors-Fressenborg and U. Jöhncke (1994) The assessment of bioaccumulation. Chemosphere 29, pp. 15011514.

Grosjean D and Grosjean E (1994). in Amer Chem Soc, Div Environm Chem Preprint. 207th ACS Natl. Mtg., 34: 705-7

Hansch, C., Leo, A., and Hoekman, D. (1995) Exploring QSAR. Hydrophobic, Electronic, and Steric Constants. ACS Prof Ref Book. Heller SR, consult. ed., Washington, DC: Amer Chem Soc p. 6.

Knoevenagel K and Himmelreich R (1976). Degradation of compounds containing carbon atoms by photooxidation in the presence of water. Arch Environ Contam 4: 324-33

Loehr RC. (1989). Treatability Potential for EPA Listed Hazardous Wastes in Soil. Ada, OK: USEPA. Robert S. Kerr Environ. Res. Lab., USEPA/60o/2-89/o11.

Meylan, W.M., P.H. Howard and R.S. Boethling. (1992). Molecular topology/fragment contribution method for predicting soil sorption coefficients. Environ Sci Technol 26: $1560-67$.

Meylan, W. M. and P. H. Howard (1993). Reliability: Estimate based on known qualitative structure-activity relationships Chemosphere, 26:2293-99.

Meylan, WM; Howard, PH; and Boethling, RS. (1999). Improved method for estimating bioconcentration/bioaccumulation factor from octanol/water partition coefficient. Environ. Toxicol. Chem. 18: 664-672.

Scheunert I, Vockel D, Klein W, and Korte F (1981). Fate of 14C-allylalcohol herbicide in soils and crop residues; J Environ Sci Health 6: 719-42.

Swann, R.L., Laskowski, D.A., McCall, P.J., van der Kuy, K. and Dishburger, H.J. (1983). A rapid method for estimation of the environmental parameters octanol/water partition coefficient, soil sorption constant water to air ratio and water solubility. Residue Rev. 85, pp. 17-28. (http://toxnet.nlm.nih.gov/cgi-bin/sis/search/r?dbs+hsdb:@term+@rn+6o$34-4)$. 


\title{
Carbon disulfide $75^{-15}-0$
}

\section{ENVIRONMENTAL TRANSPORT, DISTRIBUTION, AND TRANSFORMATION}

\begin{abstract}
Air: In air, carbon disulfide is primarily degraded through photo-oxidation by reactions with hydroxyl $(\mathrm{OH})$ radicals and by a secondary route involving triplet oxygen $\left(\mathrm{O}\left({ }_{3} \mathrm{P}\right)\right)$. With a hydroxyl radical concentration of $5 \times 10^{5}$ radicals $/ \mathrm{cm}^{3}$, a half-life of about 5.5-15 days is calculated from rate constants between $1.1 \times 10^{-12}$ and $2.9 \times 10^{-12} \mathrm{~cm} 3 /$ molecule per second (BUA, 1993). Wine et al. (1981) likewise estimated that photo-oxidation in the troposphere results in a half-life in air of 7-14 days. Reaction products include carbonyl sulfide (COS) and sulfur dioxide $\left(\mathrm{SO}_{2}\right)$. Carbonyl sulfide has a much longer lifetime (2 years) than carbon disulfide in the atmosphere.
\end{abstract}

Photolysis of carbon disulfide by radiation at wavelengths above $290 \mathrm{~nm}$ occurs in the troposphere. An atmospheric lifetime of 11 days (half-life of 7.7 days) was calculated assuming $12 \mathrm{~h}$ of sunlight (Peyton et al., 1976). Wood and Heicklen (1971) demonstrated that direct photolysis of carbon disulfide at $313 \mathrm{~nm}$ produces reaction products similar to those of the photo-oxidation reaction - that is, carbon monoxide (CO), carbonyl sulfide, sulfur dioxide plus an unidentified polymeric material. Wet deposition from the atmosphere is probably a minor removal process, because carbon disulfide is interacted only weakly with water (Lovejoy, 1989).

Water: With a Henry's law constant of $1748 \mathrm{~Pa} \mathrm{~m} 3 / \mathrm{mol}$ at $20^{\circ} \mathrm{C}$ and a vapor pressure of $48.2 \mathrm{kPa}$ at $20^{\circ} \mathrm{C}$, the major fate process for carbon disulfide released into water is volatilization, with a half-life ranging between 11 minutes in water (saturated solution) and 2.6 hours in a model river (Peyton et al., 1976; Howard, 1989). Carbon disulfide is resistant to hydrolysis in water within the biological $\mathrm{pH}$ range (4-10), with a hydrolysis half-life extrapolated to $\mathrm{pH} 9$ of 1.1 years (Peyton et al., 1976). Its predicted rate of biodegradation in water is negligible compared with its rate of volatilization from surface water (ATSDR, 1996). The mean degradation half-life used for fugacity modeling by DMER and AEL (1996) of 5500 hours (7.4 months) was based on the estimate of biodegradation half-life by Abrams et al. (1975).

Sediment: Owing to its low affinity for sorption to organic substances (organic carbon/water partition coefficient $[\log K o c]=1.79$ ), very little carbon disulfide is likely to partition to or remain in sediment. One study indicated that the soil/sediment microorganism Thiobacillus thiorapus (grown aerobically, incubated anaerobically) was able to metabolize carbon disulfide to produce carbonyl sulfide and hydrogen sulfide (Smith and Kelly, 1988). Thus, some biodegradation is expected to occur. The estimated 
mean reactivity half-life used for fugacity modeling was 5500 hours (7.4 months), based on the estimate of biodegradation half-life by Abrams et al. (1975).

Soils: No estimates of a half-life for carbon disulfide in soil were identified in the literature. Aerobic degradation of carbon disulfide has been observed with a strain of Thiobacillus thiorapus. This particular strain was able to hydrolytically oxidize carbon disulfide sequentially to carbonyl sulfide and hydrogen sulfide; all the carbon was released as carbon dioxide, followed by oxidation of the sulfide to sulfate (Smith and Kelly, 1988). For soil, DMER and AEL (1996) used a mean degradation half-life of 5500 hours for their fugacity modeling, based on the estimate of biodegradation half-life by Abrams et al. (1975). In the natural environment, carbon disulfide is highly mobile in soil ( $\log \mathrm{Koc}=1.79)$ and is subject to rapid volatilization, so it is unlikely to remain in soil long enough to undergo significant biodegradation.

Biota: Carbon disulfide is expected to have little or no tendency to bioaccumulate or biomagnify in biota, owing to its relatively low octanol/water partition coefficient (log Kow) value (2.14) and rapid metabolism in most animals (Beauchamp et al., 1983).

Environmental Partitioning: Fugacity modeling was carried out to provide an overview of key reaction, intercompartment and advection (movement out of a system) pathways for carbon disulfide and its overall distribution in the environment (DMER and AEL, 1996). A steady-state, non-equilibrium EQC model (Level III fugacity modeling) was run using the methods developed by Mackay (1991) and Mackay and Paterson (1991). Values for input parameters were as follows: molecular weight, $76.1 \mathrm{~g} / \mathrm{mol}$; water solubility, $2100 \mathrm{mg} / \mathrm{L}$; vapor pressure, 48 210 Pa; log Kow, 2.14; Henry's law constant, $1748 \mathrm{~Pa} \mathrm{~m} 3 / \mathrm{mol}$; half-life in air, 170 hours; half-life in water, soil and sediment, 5500 hours. Modeling was based on an assumed default emission rate of $1000 \mathrm{~kg} / \mathrm{hour}$ into a region of $100000 \mathrm{~km}^{2}$, which includes a $10000-\mathrm{km}^{2}$ area of surface water (20 m deep). The height of the atmosphere is $1000 \mathrm{~m}$. Sediments and soils have an organic carbon content of $4 \%$ and $2 \%$ and a depth of $1 \mathrm{~cm}$ and $10 \mathrm{~cm}$, respectively. The estimated percent distribution predicted by this model is not affected by the assumed emission rate.

Modeling indicates that carbon disulfide partitions differently depending on the medium to which it is released. For example, if emitted into air, $99.8 \%$ of the carbon disulfide is present in air; if emitted into soil, the fraction in air is reduced to 73\%, with most of the rest in soil. When carbon disulfide is released to water, it is present primarily in water (85\%) and, to a lesser extent, in air (15\%) (DMER and AEL, 1996). Thus, while the predicted distributions suggest that little intermedia transport will occur when carbon disulfide is discharged to air, release to each of soil and (to a lesser extent) water has the potential for substantial transport of carbon disulfide to air. 


\section{Regulatory}

MCL (Drinking Water): None

GPS - Groundwater protection standard (DWEL): 3,650 ug/L

Universal Treatment Standards (UTS): $3.8 \mathrm{mg} / \mathrm{l2}$ in wastewater standards and 4.8mg/L TCLP

Controlling Water Quality Criterion for Surface Water: (WDEQ)

Basis: Aquatic Toxicity Criterion Concentration: $21 \mathrm{ug} / \mathrm{L}$ Criterion Quantitation Limit: 5 ug/L

EPA Rf D: $0.1 \mathrm{mg} / \mathrm{kg}$-day

EPA RfC: $0.7 \mathrm{mg} / \mathrm{m} 3$

PRG: residential soil mg/kg industrial soil $\mathrm{mg} / \mathrm{kg}$ ambient air ug/m tap water $\mathrm{ug} / \mathrm{L}$

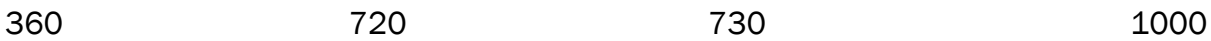

\section{Remediation}

Remove all ignition sources. Ventilate area of spill or leak. For small quantities, absorb on paper towels and evaporate in a safe place (such as a fume hood). Allow sufficient time for evaporating of vapors to completely clear the hood ductwork. Burn the paper in a suitable location away from combustible materials. Large quantities can be reclaimed or collected and atomized in a suitable combustion chamber equipped with an appropriate effluent gas cleaning device. Carbon disulfide should not be allowed to enter a confined space, such as a sewer, because of the possibility of an explosion.

\section{References}

Abrams, E.F., D. Derkics, C.V. Fong, D.K. Guinan and K.M. Slimak. (1975). Identification of organic compounds in effluents from industrial sources. U.S. Environmental Protection Agency (EPA-560/3-75-002; PB 241641 OBA).

ATSDR, Agency for Toxic Substances and Disease Registry (1996). Toxicological profile for carbon disulfide (update). Public Health Service, U.S. Department of Health and Human Services, Atlanta, Georgia. 219. 
Beauchamp, R.O., J.S. Bus, J.A. Popp, C.J. Boreiko and L. Glodberg. (1983). A critical review of the literature on carbon disulfide toxicity. CRC Crit. Rev. Toxicol. 11: 169-278.

BUA (Beratunggremium für Umweltrelevante Alstoffe). (1993). Carbon disulfide. GDCh Advisory Committee on Existing Chemicals of Environmental Relevance. S. Hirzel Verlag, Stuttgart, Germany (Report No. 83).

DMER and AEL (Don Mackay Environmental Research and Angus Environmental Limited) (1996). Pathways analysis using fugacity modeling of carbon disulfide for the second Priority Substances List. DMER, Peterborough, Ontario, and AEL, Don Mills, Ontario.

Environment Canada Health Canada (2000) ISBN: 0-662-28496-8 Cat. No.: En40215/46E Canadian Environmental Protection Act, 1999.

Howard, P. (1989). Handbook of environmental fate and exposure data for organic chemicals. Vol. 2: Solvents. Lewis Publishers, Boca Raton, Florida.

Lovejoy, E.R. (1989). The kinetics and products of the OH initiated oxidation of CS2 and O2. Ph.D. thesis, University of Colorado, Boulder, Colorado.

Mackay, D. (1991). Multimedia environmental models: the fugacity approach. Lewis Publishers, Chelsea, Michigan.

Mackay, D. and S. Paterson. (1991). Evaluating the multimedia fate of organic chemicals: a Level III fugacity model. Environ. Sci. Technol. 25: 427-436.

Mackison, F. W., R. S. Stricoff, and L. J. Partridge, Jr. (eds.). NIOSH/OSHA Occupational Health Guidelines for Chemical Hazards. DHHS(NIOSH) Publication No. 81-123 (3 VOLS). Washington, DC: U.S. Government Printing Office, Jan. 1981, p. 4.

NPRI, National Pollutant Release Inventory. (1996a). Summary report 1995, National Pollutant Release Inventory, Canadian Environmental Protection Act. Environment Canada, Hull, Quebec.

Peyton, T.O., R.V. Steele and W.R. Mabey. 1976. Carbon disulfide, carbonyl sulfide: literature review and environmental assessment. U.S. Environmental Protection Agency (EPA-600/9-78-009). 
Smith, N.A. and D.P. Kelly. (1988). Oxidation of carbon disulfide as the sole source of energy for the autotrophic growth of Thiobacillus thiorapus. Strain TK-m. J. Gen. Microbiol. 134: 3041-3048.

Wine, P.H., W.L. Chameides and A.R. Ravishankara. (1981). Potential role of CS photooxidation in tropospheric sulfur chemistry. Geophys. Res. Lett. 8(5): 543-546.

Wood, W.P. and J. Heicklen. (1971). The photooxidation of carbon disulfide. J. Phys. Chem. 75: 854-860.

Wyoming Department of Environmental Quality, Water Quality Division, Chapter 17; 12/01/o5. 


\title{
Chloroethylene (Vinyl Chloride) 75-01-4
}

\section{ENVIRONMENTAL TRANSPORT, DISTRIBUTION, AND TRANSFORMATION}

\begin{abstract}
Air: According to a model of gas/particle partitioning of semivolatile organic compounds in the atmosphere (Bidleman 1988), vinyl chloride, which has a vapor pressure of 2,980 $\mathrm{mm} \mathrm{Hg}$ at $25 \operatorname{deg~C~(Daubert~and~Danner~1989),~is~expected~to~exist~solely~as~a~gas~in~}$ the ambient atmosphere. Gas-phase vinyl chloride is degraded in the atmosphere by reaction with photochemically-produced hydroxyl radicals (SRC); the half-life for this reaction in air is estimated to be 55 hours, calculated from its rate constant of $6.96 \mathrm{X}_{10} \mathrm{O}^{-12}$ $\mathrm{cu} \mathrm{cm} /$ molecule-sec at 25 deg C (Atkinson 1989). Vinyl chloride is not expected to undergo considerable direct photolysis since this compound does not absorb light appreciably in the environmental UV spectrum.
\end{abstract}

Water: Based on a classification scheme (Swann et al 1983), an estimated Koc value of 57 (SRC), calculated from a water solubility of $2,700 \mathrm{mg} / \mathrm{l}$ (Riddick et al 1986) and a regression derived equation (Lyman et al 1990) indicates that vinyl chloride is not expected to adsorb to suspended solids and sediment in water (SRC). Volatilization from water surfaces is expected to occur rapidly (Lyman et al 1990) based upon a Henry's Law constant of 0.0278 atm-cu m/mole (Gossett 1987). Using this Henry's Law constant and an estimation method (Lyman et al 1990), volatilization half-lives for a model river and model lake are 1 hour and 3 days, respectively (SRC). According to a classification scheme (Franke et al 1994), a BCF value of less than 10 measured in fish (Freitag et al 1985), suggests bioconcentration in aquatic organisms is low(SRC). The biodegradation half-life of vinyl chloride in aerobic and anaerobic waters was reported as 28 and 110 days, respectively (Capel and Larson 1995). Hydrolysis is not expected to be an important environmental fate process based on hydrolysis half-lives of 9.91 years ( $\mathrm{pH}=7,25 \mathrm{deg} \mathrm{C}$ ) and 10.7 years $(\mathrm{pH}=7,10 \mathrm{deg} \mathrm{C})$. Vinyl chloride may undergo indirect photolysis in natural waters when photosensitizers such as humic material are available (Mill 1999).

Soil/Sediment: An estimated Koc value of $57($ SRC), calculated from a water solubility of 2,700 mg/l (Riddick et al 1986) and a regression derived equation (Lyman et al 1990) indicates that vinyl chloride is expected to have high mobility in soil (Swann et al 1983). Volatilization of vinyl chloride from moist soil surfaces is expected to be an important fate process (SRC) given a Henry's Law constant of 0.0278 atm-cu m/mole (Gossett 1987). Vinyl chloride may volatilize from dry soil surfaces based on a vapor pressure of 2,780 $\mathrm{mm} \mathrm{Hg}$ at 25 deg C (Daubert and Danner 1989). The volatilization half-life of vinyl chloride was estimated as 0.2 days when incorporated in a soil at a depth of $1 \mathrm{~cm}$ and 0.5 days at a depth of $10 \mathrm{~cm}$ (Jury et al 1984). 
Biota: Some /data indicated/ that vinyl chloride is too readily volatilized to undergo bioaccumulation, except perhaps in the most extreme exposure conditions. Studies on five bacterial, three fungal, and two single organism cultures from natural aquatic systems did not show bioaccumulation to be an appreciable process (Callahan et al 1979). The BCF value of vinyl chloride in golden ide fish was reported as less than 10 (Freitag et al 1985). The BCF value of vinyl chloride in green algae was reported as 40. According to a classification scheme (Franke et al 1994), the BCF data suggest that bioconcentration in aquatic organisms is low (SRC).

\section{Regulations}

MCL (Drinking Water): $0.002 \mathrm{mg} / \mathrm{L}$

GPS - Groundwater protection standard (DWEL): $0.002 \mathrm{mg} / \mathrm{L}$

Universal Treatment Standards (UTS): $0.27 \mathrm{mg} / \mathrm{l} 2$ in wastewater standards and 6.0 $\mathrm{mg} / \mathrm{kg} 3$

Groundwater Water Quality Criterion : 0.27ug/L

PQL: 1ug/L

EPA Rf D: $0.002 \mathrm{mg} / \mathrm{kg}$-day

EPA RfC: $0.1 \mathrm{mg} / \mathrm{m}^{3}$

PRG: residential soil $\mathrm{mg} / \mathrm{kg}$ industrial soil $\mathrm{mg} / \mathrm{kg} \quad$ ambient air $\mathrm{ug} / \mathrm{m} \quad$ tap water $\mathrm{ug} / \mathrm{L}$ $\begin{array}{llll}0.15 & 0.83 & 0.22 & 0.041\end{array}$

\section{Remediation}

Land Spill: Construct barriers to contain spill. Absorb small amounts of spill with natural or synthetic sorbents, shovel into containers with covers.

Water Spill: Contain contaminated water with dams or natural barriers. (Environment Canada)

\section{References}

Atkinson R; (1989) Kinetics and mechanisms of the gas-phase reactions of the hydroxyl radical with organic compounds. J Phys Chem Ref Data Monograph 1. 
Bidleman T. F. (1988) Atmospheric processes. Envir. Sei. Technol. 22:361-367.

Callahan, M.A., M.W. Slimak, N.W. Gabel; (1979). Water-Related Environmental Fate of 129 Priority Pollutants. Volume II. EPA-440/4-79-029b. Washington, D.C.: U.S. Environmental Protection Agency, p. 49-6.

Capel, P.D., and Larson, S.J., 1995, A Chemodynamic Approach for Estimating Losses of Target Organic Chemicals from Water During Sample Holding Time: Chemosphere, v. 30, no. 6, p. 1097-1107.

Daubert TE, Danner RP; (1989) Physical and Thermodynamic Properties of Pure Chemicals Data Compilation Washington, DC: Taylor and Francis.

Environment Canada; Tech Info for Problem Spills: Vinyl Chloride (Draft) p.2 (1980) Freitag, D., Ballhorn, L., Geyer, H. Korte, F., (1985) Environmental hazard profile of organic chemicals. Chemosphere 14: 1589-1616.

Franke, C., G. Studinger, G. Berger, S. Böhling, U. Bruckmann, D. Cohors-Fressenborg and U. Jöhncke (1994) The assessment of bioaccumulation. Chemosphere 29: 1501-14

Gossett, J.M., (1987) Measurement of Henry's Law Constants for C1 and C2 Chlorinated Hydrocarbons, Environmental Science \& Technology, 21, 202-208.

Jury WA, Spencer WF, Farmer WJ. (1984) Behavior assessment model for trace organics in soil: III. Application of screening model. J. Environ Qual; 13:573-79.

Lyman, WJ, WF Reehl, and DH Rosenblatt (1990) Handbook of Chemical Property Estimation Methods. Washington, DC: American Chemical Society pp. 4-5, 15-1 to 15-29.

Mill T; (1999). Predicting photoreaction rates in surface waters.Chemosphere 38: 137990.

Riddick JA, Bunger WB, Sakano TK. (1986). Organic solvents: Physical properties and methods of purification. Techniques of chemistry. 4th ed. New York, NY: Wiley-Interscience, 1325.

Swann, R.L., Laskowski, D.A., McCall, P.J., van der Kuy, K. and Dishburger, H.J. (1983) A rapid method for estimation of the environmental parameters octanol/water partition coefficient, soil sorption constant water to air ratio and water solubility. Residue Rev. 85, pp. 17-28. (http://toxnet.nlm.nih.gov/cgi-bin/sis/search/r?dbs+hsdb:@term+@rn+6o34-4). 


\title{
Chlorohydrin (3-Chloro-1,2-Propanediol) 96-24-2
}

\section{ENVIRONMENTAL TRANSPORT, DISTRIBUTION, AND TRANSFORMATION}

\author{
Air: According to a model of gas/particle partitioning of semivolatile organic compounds \\ in the atmosphere (Bidleman 1988), 3-chloro-1,2-dihydroxypropane, which has a \\ measured vapor pressure of $0.2 \mathrm{~mm} \mathrm{Hg}$ at $20 \mathrm{deg}$ C (Lande 1980), will exist solely as a \\ vapor in the ambient atmosphere. Vapor-phase 3-chloro-1,2-dihydroxypropane is \\ degraded in the atmosphere by reaction with photochemically produced hydroxyl \\ radicals(SRC); the half-life for this reaction in air is estimated to be about 2 days \\ (Meylan and Howard 1993).
}

Water: Based on a recommended classification scheme (Lyman et al.1990), an estimated Koc value of 1(SRC), determined from structure estimation method (Meylan et al. 1992), indicates that 3-chloro-1,2-dihydroxypropane should not adsorb to suspended solids and sediment in water (SRC). 3-Chloro-1, 2-dihydroxypropane is not expected to volatilize from water surfaces (Lyman et al. 1990, SRC) based on an estimated Henry's Law constant of 6.1X10-8 atm-cu m/mole (SRC), developed using a fragment constant estimation method (Meylan and Howard 1991). According to a classification scheme (Franke et al. 1994), an estimated BCF value of 0.2 (Lyman et al. 1990, SRC), from an estimated log Kow (Meylan and Howard 1995, SRC), suggests that bioconcentration in aquatic organisms is low (SRC). Data regarding the aerobic biodegradation of 3 -chloro1,2-dihydroxypropane in water are inconclusive; in screening tests, this compound reached o(Niemi et al. 1987) to $1 \%$ (Bridie et al. 1979) of the theoretical BOD in 5 days and $68 \%$ of the theoretical BOD in 14 days (Chemicals Inspection and Testing Institute). Under anaerobic conditions, 3-chloro-1,2-dihydroxypropane may be resistant to biodegradation; using an acclimated culture, only $26 \%$ of the initial 3-chloro-1,2dihydroxypropane concentration was utilized in 90 days (Chou et al. 1987).

Soil: An estimated Koc value of 1 (SRC), determined from a structure estimation method (Meylan et al. 1992), indicates that 3-chloro-1,2-dihydroxypropane will have very high mobility in soil (Swann et al. 1983). Volatilization of 3-chloro-1,2-dihydroxypropane is not expected to be significant from moist soil surfaces (SRC) given an estimated Henry's Law constant of $6.1 \mathrm{X}_{10} \mathrm{O}^{-8} \mathrm{~atm}-\mathrm{cu} \mathrm{m} / \mathrm{mole}$ (SRC), using a fragment constant estimation method (Meylan and Howard 1991). Data regarding the aerobic biodegradation of 3chloro-1, 2-dihydroxypropane in soil are variable; in screening tests, this compound reached o (Niemi et al. 1987) to 1\% (Bridie et al. 1979) of the theoretical BOD in 5 days and $68 \%$ of the theoretical BOD in 14 days (Chemicals Inspection and Testing Institute). Under anaerobic conditions, 3-chloro-1, 2-dihydroxypropane may be resistant to 
biodegradation. Using an acclimated culture, only $26 \%$ of the initial 3-chloro-1,2dihydroxypropane concentration was utilized in 90 days (Chou et al. 1978).

Biota: An estimated BCF value of 0.2 was calculated for 3-chloro-1,2-dihydroxypropane (SRC), using an estimated log Kow of -0.53 (Meylan and Howard) and a recommended regression-derived equation (Lyman et al . 1990). According to a classification scheme (Franke et al. 1994), this BCF value suggests that bioconcentration in aquatic organisms is low.

\section{Regulatory}

MCL (Drinking Water): none

Universal Treatment Standards (UTS): none

EPA RfD: discontinued May 1995

EPA RfC: discountinued May 1995

IDLH: $10 \mathrm{mg} / \mathrm{m}^{3}$

\section{Remediation}

Criteria for land treatment or burial (sanitary landfill) disposal practices are subject to significant revision. Prior to implementing land disposal of waste residue (including waste sludge), consult with environmental regulatory agencies for guidance on acceptable disposal practices.

\section{References}

Bidleman, T.F. (1988). Atmospheric processes: Great Lakes Pesticide Report, Environmental Science and Technology. 22(4):361-367.

Bridie, AL, Wolff, CJM, and Winter, M (1979a). BOD and COD of some petrochemicals. Water Research 13, 627-630.

Chemicals Inspection and Testing Institute; (1992). Biodegradation and Bioaccumulation Data of Existing Chemicals Based on the CSCL Japan. Japan Chemical Industry EcologyToxicology and Information Center. ISBN \#4-89074-101-1. 
Chou, W.L., Speece, R.E., and Siddiqi, R.H. (1978). Acclimation and degradation of ... In: Biotechnology and Bioengineering Symposium No. 8., C.D. Scott, ed., pp. 391-414. John Wiley and Sons, New York, NY.

Franke, C., G. Studinger, G. Berger, S. Böhling, U. Bruckmann, D. Cohors-Fressenborg and U. Jöhncke, (1994).The assessment of bioaccumulation. Chemosphere 29: 1501-14.

Lande SS (1980); Investigation of Selected Potential Environmental Contaminants: Haloalcohols. USEPA-560/11-80-004. Washington, DC: USEPA.

Lyman, WJ, WF Reehl, and DH Rosenblatt; (1990).Handbook of Chemical Property Estimation Methods. Washington, DC: Amer Chem Soc pp. 4-9, 15-1 to 15-29.

Meylan, W.M. and Howard, P.H. (1991). Bond contribution method for estimating Henry's Law Constants. Environ. Toxicol. Chem. 10: 1283-93.

Meylan WM, P.H. Howard, and R.S. Boethfing. (1992). Molecular topology/fragment contribution method for predicting soil sorption coefficients. Environ Sci Technol 28: $459-65$.

Meylan, W. M. and P. H. Howard (1993). Reliability: Estimate based on known qualitative structure-activity relationships Chemosphere, 26:2293-99.

Meylan WM and Howard PH (1995). Atom/fragment contribution method for estimating octanol-water partition coefficients. J. Pharm. Sci. 84: 83-92.

Niemi GJ, Veith GD, Regal RR, Vaishnav DD (1987). Structural features associated with degradable and persistent chemicals. Environ. Toxicol. Chem. 6: 515-27.

Swann RL, Laskowski DA, McCall PJ, (1983). A rapid method for the estimation of the environmental parameters octanol/water partition coefficient, soil sorption constant, water to air ratio and water solubility. Res Rev 85:17-28. 


\title{
Cyanogen Chloride (CK) $\quad 506-77-4$
}

\section{ENVIRONMENTAL TRANSPORT, DISTRIBUTION, AND TRANSFORMATION}

\begin{abstract}
Air: Because hydrogen cyanide is a gas and has a relatively slow degradation rate in air, the atmosphere will be the ultimate sink for this compound. Almost all of the hydrogen cyanide released to the atmosphere remains in the lower altitudes (troposphere); only $2 \%$ of tropospheric hydrogen cyanide is transferred to the stratosphere (Cicerone and Zellner 1983). Cyanide has the potential to be transported over long distances from its emission source. Despite higher water solubility at saturated pressure, the removal of hydrogen cyanide by rainwater appears to be a negligible partitioning pathway (Cicerone and Zellner 1983). Because hydrogen cyanide is a gas, its removal from air by dry deposition is also likely to be negligible. However, metal cyanide particles, particularly water-soluble cyanide particles, are expected to be removed from the air by both wet and dry deposition.
\end{abstract}

Water: Volatilization and sorption are the two physical processes that contribute to the loss of cyanide from water. At $\mathrm{pH}<9.2$, most of the free cyanide in solution should exist as hydrogen cyanide, a volatile cyanide form (EPA 1978). On the basis of Henry's law constant and the volatility characteristics associated with various ranges of Henry's law constant (Thomas 1982), volatilization is a significant and probably dominant fate process for hydrogen cyanide in surface water (EPA 1992). The most common alkali metal cyanides (e.g., sodium and potassium cyanide) may also be lost from surface water primarily through volatilization; whereas, the sparingly soluble metal cyanides such as copper (I) cyanide are removed from water predominantly by sedimentation and biodegradation (EPA 1992). Variations in the volatilization rate are expected because this process is affected by several parameters including temperature, $\mathrm{pH}$, wind speed, and cyanide concentration (EPA 1979). EPA (1979) summarized the unpublished results of a laboratory study that indicated that the volatilization half-life of hydrogen cyanide from solutions at concentrations of $25^{-200 ~} \mu \mathrm{g} / \mathrm{L}$ ranged from 22 to 110 hours. First-order kinetics were observed. In outdoor experiments with moderate winds, the rate of hydrogen cyanide loss increased by a factor of 2-2.5. In a study to evaluate the effect of cyanide on biochemical oxidation, there was a 50\% loss of $6 \mathrm{ppm}(\mathrm{mg} / \mathrm{L})$ cyanide in river water kept in open biochemical oxygen demand bottles (without aeration) at $\mathrm{pH} 7.4$ within $\approx 10$ days. When the bottles were aerated (rate of aeration not given), $50 \%$ loss occurred in $\approx 10$ hours. The kinetics of the rate of loss due to volatilization was not rigorously investigated. The volatilization rate was $\mathrm{pH}$-dependent, with the rate faster at a lower $\mathrm{pH}$. Data indicated that cyanide volatilization is a more important fate process than cyanide loss due to chemical and biodegradation reactions (Raef et al. 1977a). Because 
volatilization is not an important fate process for cyanide in groundwater, cyanide would be expected to persist for considerably longer periods of time in underground aquifers than in surface water.

Sediment: Cyanides are sorbed by various natural media, including clays (Cruz et al. 1974), biological solids (Raef et al. 1977b), and sediments (EPA 1979). However, additional data are necessary to assess the significance of cyanide sorption to suspended solids and sediments in water. Hydrogen cyanide and the alkali metal cyanides are not likely to be strongly sorbed onto sediments and suspended solids because of their high water solubility. Soluble metal cyanides may show somewhat stronger sorption than hydrogen cyanide, with the extent of sorption increasing with decreasing $\mathrm{pH}$ and increasing iron oxide, clay, and organic material contents of sediment and suspended solids (EPA 1979). However, sorption is probably insignificant even for metal cyanides when compared to volatilization and biodegradation (EPA 1979, 1992).

Soil: Volatilization of hydrogen cyanide would be a significant loss mechanism for cyanides from soil surfaces at a $\mathrm{pH}<9.2$. Cyanides are fairly mobile in soil. Mobility is lowest in soils with low $\mathrm{pH}$ and high concentrations of free iron oxides, positively charged particles, and clays (e.g., chlorite, kaolin, gibbsite), and highest in soils with high $\mathrm{pH}$, high concentrations of free $\mathrm{CaCO} 3$ and negatively charged particles, and low clay content (EPA 1979). Although cyanide has a low soil sorption capability, it is usually not detected in groundwater, probably because of fixation by trace metals through complexation or transformation by soil microorganisms (EPA 1978). In soils where cyanide levels are high enough to be toxic to microorganisms (i.e., landfills, spills), this compound may leach into groundwater (EPA 1984). Also, leaching of cyanide into a shallow aquifer can occur, as demonstrated by the high concentration of cyanide $(1,200 \mu \mathrm{g} / \mathrm{L})$ in groundwater sampled from the Biscayne Aquifer in Dade County, Florida, which lies below a solid waste site (Myers 1983).

Biota: There are no data available to indicate that simple metal cyanides and hydrogen cyanide bioconcentrate in aquatic organisms (EPA 1979, 1980, 1985, 1992).

Bioconcentration factors (BCFs) of 0.73 and 1.62 can be calculated for hydrogen cyanide, using the equation of Veith et al. (1979) for the BCF of a chemical in whole fish (log BCF, 0.85; log Kow, -0.70) and the log Kow values. Similarly, the calculated BCF for sodium cyanide is 0.47 . There is some evidence that certain metal cyanide complexes bioaccumulate in aquatic organisms. Fish from water with soluble silver and copper cyanide complexes were found to have metal cyanides in their tissues at concentrations ranging up to 168 and $304 \mu \mathrm{g} / \mathrm{g}$, respectively (wet or dry weight not specified) (EPA 1979). It is difficult to evaluate the toxicologic significance of bioaccumulation of metal cyanide complexes because these compounds are much less toxic than soluble hydrogen cyanide, sodium cyanide, or potassium cyanide (EPA 1992). There is no evidence of 
biomagnification of cyanides in the food chain (EPA 1978). Accumulation of cyanide in food webs is not expected, considering the rapid detoxification of cyanide by most species and the lethal effects of large doses of cyanide (EPA 1978).

Environmental partitioning: No information could be found in the available literature on the transport and partitioning of cyanogen chloride in the environment, or its partitioning coefficients (Koc, Kow) or Henry's law constants. Like cyanogen, cyanogen chloride is a highly volatile gas. Therefore, it would be expected that volatilization from water and soil would be a primary route of environmental partitioning for both cyanogen and cyanogen chloride.

\section{Regulatory}

MCL (Drinking Water) for cyanide: $0.2 \mathrm{mg} / \mathrm{L}$

Universal Treatment Standards (UTS): None established

Controlling Water Quality Criterion for Surface Water:

Basis: Aquatic Toxicity Criterion Concentration: ug/L Criterion Quantitation Limit: ug/L

EPA Rf D: $25.3 \mathrm{mg} / \mathrm{kg}$-day

EPA RfC: $3 \times 10-3 \mathrm{mg} / \mathrm{m}^{3}$

PRG: residential soil $\mathrm{mg} / \mathrm{kg}$ industrial soil $\mathrm{mg} / \mathrm{kg}$ ambient air $\mathrm{ug} / \mathrm{m} \quad$ tap water $\mathrm{ug} / \mathrm{L}$ $160 \quad 540 \quad 180 \quad 300$

Volatile Organic Cmpd. Measured using Method 8260: Permit limit 2000 ug/L

\section{Remediation}

Land spill: Dig a pit, pond, lagoon, or holding area to contain liquid or solid material. SRP: If time permits, pits, ponds, lagoons, soak holes, or holding areas should be sealed with an impermeable flexible membrane liner. Dike surface flow using soil, sand bags, foamed polyurethane, or foamed concrete. Absorb bulk liquid with fly ash or cement powder. /Cyanogen chloride, inhibited/

Water spill: Add dilute caustic soda. Add calcium hypochlorite. Adjust $\mathrm{pH}$ to neutral $\mathrm{pH}$ 7). Cyanogen chloride, inhibited.

Air spill: Apply water spray or mist to knock down vapors. Vapor knock down water is corrosive or toxic and should be diked for containment (Association of American Railroads). 


\section{References}

Association of American Railroads; (2005) Bureau of Explosives. Emergency Handling of Hazardous Materials in Surface Transportation., Pueblo, CO., p. 267.

Brown, P.D. and M.J. Morra. (1993). Fate of ionic thiocyanate (SCN-) in soil. J. Agric. Food Chem. 41:978-982.

R.J. Cicerone and R. Zellner (1983). The atmospheric chemistry of hydrogen cyanide (HCN). J. Geophys. Res. 88, pp. 10689-10696.

Cruz, M., A. Kaiser, P.G. Rouxhet, and J.J. Fripiat. 1974. Adsorption and transformation of HCN on the surface of copper and calcium montmorillonite. Clays and Clay Minerals 22:417-425.

EPA. (1984). Health Assessment Document for Arsenic. Research Triangle Park, NC: U.S. Environmental Protection Agency. EPA600823021F.

EPA. (1978). Reviews of the environmental effects of pollutants. V. Cyanide. Cincinnati, OH: U.S. Environmental Protection Agency Health Effects Research Laboratory, Office of Research and Development. PB289920.

EPA. (1979). Cyanides. In: Water-related environmental fate of 129 priority pollutants. Vol. 1. Washington, DC: U.S. Environmental Protection Agency, Office of Water Planning and Standards, Office of Water and Waste Management. EPA440479029a. PB80204373. 12-1-12-12.

EPA. (1984). Health effects assessment for cyanide. Washington, DC: U.S. Environmental Protection Agency. EPA540186011.

EPA. (1992). U.S. Environmental Protection Agency. Fed Regist 57:26248.

Myers VB. (1983). Remedial activities at the Miami Drum site, Florida. In: National conference on management of uncontrolled hazardous waste sites. Silver Springs, MD: Hazardous Materials Control Research Institute, 354-357.

Raef SF, Characklis WG, Kessick MA. (1977a). Fate of cyanide and related compounds in aerobic microbial systems--II. Microbial degradation. Water Res 11:485-492. 
Raef SF, Characklis WG, Kessick MA (1977b). Fate of cyanide and related compounds in aerobic microbial systems. I. Chemical reaction with substrate and physical removal. Water Res 11:477-483.

Thomas RG. (1982). Volatilization from water. In: Lyman WJ, Reehl, WF, Rosenblatt DH, eds. Handbook of chemical property estimation methods: Environmental behavior of organic compounds. New York, NY: McGraw-Hill Book Co., 15-16. 


\title{
Dimethylamine 124-40-3
}

\section{ENVIRONMENTAL TRANSPORT, DISTRIBUTION, AND TRANSFORMATION}

\begin{abstract}
Air: According to a model of gas/particle partitioning of semivolatile organic compounds in the atmosphere (Bidleman 19881), dimethylamine, which has a vapor pressure of 1,520 $\mathrm{mm} \mathrm{Hg}$ at 25 deg C (Daubert and Danner 1985), is expected to exist solely as a gas in the ambient atmosphere. Gas-phase dimethylamine is degraded in the atmosphere by reaction with photochemically-produced hydroxyl radicals(SRC); the half-life for this reaction in air is estimated to be 6 hours (SRC), calculated from its rate constant of 6.54X10-11 $\mathrm{cu} \mathrm{cm} / \mathrm{molecule-sec}$ at $25 \mathrm{deg} \mathrm{C}$ (Atkinson 1989). Dimethylamine does not contain chromophores that absorb at wavelengths $>290 \mathrm{~nm}$ (Lyman et al 1990) and therefore is not expected to be susceptible to direct photolysis by sunlight (SRC).
\end{abstract}

Water: A Koc value of 508 in lake sediment (von Oepen et al 1991), indicates that dimethylamine is expected to adsorb to suspended solids and sediment (Swann et al 1983). A pKa of 10.73 (Perrin 1965) indicates diemthylamine will exist almost entirely in the cation form at $\mathrm{pH}$ values of 5 to 9 and therefore volatilization from water surfaces is not expected to be an important fate process (Doucette 2000). According to a classification scheme (Franke et al 1994), an estimated BCF of 3 (SRC), from its log Kow of -0.38 (Hansch et al 1995) and a regression-derived equation (Meylan et al 1999), suggests the potential for bioconcentration in aquatic organisms is low (SRC). Dimethylamine is expected to biodegrade in water surfaces based on a half-life of 1.6 days in Vistula River water (Warsaw, Poland) following a 0.3 day lag period (Dojlido 1979).

Soil/Sediment: Based on a classification scheme (Swann et al 1983), an average Koc value of 434.9 calculated from data of 5 soils (Rao and Davidson 1982), indicates that dimethylamine is expected to have moderate mobility in soil (SRC). The pKa of dimethylamine is 10.73 (Perrin 1965), indicating that this compound will exist almost entirely in the cation form in the environment; volatilization of dimethylamine from moist soil surfaces is not expected to be an important fate process given its cationic state (SRC). Dimethylamine is expected to volatilize from dry soil surfaces (SRC) based upon a vapor pressure of 1,520 mm $\mathrm{Hg}$ (Daubert and Danner 1985). Dimethylamine was biodegraded 69-89\% in three Saskatchewan soils during a 7 day incubation period (Smith and Aubin 1992), suggesting that biodegradation may be an important environmental fate process in soil (SRC).

Biota: An estimated BCF of 3 was calculated in fish for dimethylamine (SRC), using a log Kow of -0.38 (Hansch et al 1995) and a regression-derived equation (Meylan et al; 1999). 
According to a classification scheme (Franke et al 1994), this BCF suggests the potential for bioconcentration in aquatic organisms is low (SRC).

Environmental Partitioning: The rate constant for the vapor-phase reaction of dimethylamine with photochemically-produced hydroxyl radicals has been measured as $6.54 \mathrm{X}_{10} \mathrm{O}^{-11} \mathrm{cu} \mathrm{cm} / \mathrm{molecule-sec}$ at $25 \mathrm{deg} \mathrm{C}$ (Atkinson 1989). This corresponds to an atmospheric half-life of about $6 \mathrm{~h}$ at an atmospheric concentration of $5 \mathrm{X} 10^{5}$ hydroxyl radicals per cu cm (Atkinson 1989). Dimethylamine is not expected to undergo hydrolysis in the environment due to the lack of functional groups that hydrolyze under environmental conditions. Dimethylamine does not contain chromophores that absorb at wavelengths $>290 \mathrm{~nm}$ (Lyman et al 1990) and therefore is not expected to be susceptible to direct photolysis by sunlight.

\section{Regulations}

MCL (Drinking Water): None

GPS - Groundwater protection standard (DWEL): none

Universal Treatment Standards (UTS): none

Groundwater Water Quality Criterion: none

LAND BAN HEALTH BASED LIMITS: $0.005 \mathrm{mg} / \mathrm{L}$

EPA RfD: none

EPA RfC: none

PRG: residential soil $\mathrm{mg} / \mathrm{kg}$ industrial soil $\mathrm{mg} / \mathrm{kg}$ ambient air $\mathrm{ug} / \mathrm{m} \quad$ tap water $\mathrm{ug} / \mathrm{L}$ $\begin{array}{llll}0.067 & 0.25 & 0.021 & 0.035\end{array}$

\section{Remediation}

Land spill: Dig a pit, pond, lagoon, holding area to contain liquid or solid material. /SRP: If time permits, pits, ponds, lagoons, soak holes, or holding areas should be sealed with an impermeable flexible membrane liner. Dike the surface flow using soil, sand bags, foamed polyurethane, or foamed concrete. Absorb bulk liquid with fly ash, cement powder, or commercial sorbents. Neutralize with sodium bisulfate $\left(\mathrm{NaHSO}_{4}\right)$ 
Water spill: Add sodium bisulfate $\left(\mathrm{NaHSO}_{4}\right)$. If dissolved, in region of $10 \mathrm{ppm}$ or greater concentration, apply activated carbon at ten times the spilled amount. Use mechanical dredges or lifts to remove immobilized masses of pollutants and precipitates (Association of American Railroads).

\section{References}

Association of American Railroads; Bureau of Explosives (2005) Emergency Handling of Hazardous Materials in Surface Transportation. Association of American Railroads, Pueblo, CO., p. 339.

Atkinson R; (1989) Kinetics and mechanisms of the gas-phase reactions of the hydroxyl radical with organic compounds. J Phys Chem Ref Data Monograph 1.

Bidleman T. F. (1988) Atmospheric processes. Envir. Sci. Technol. 22:361-367.

Daubert TE, Danner RP; (1985) Physical and Thermodynamic Properties of Pure Chemicals Data Compilation Washington, DC: Taylor and Francis.

Dojlido JR; (1979) Investigations of Biodegradability and Toxicity of Organic Compounds USEPA-60o/2-79-163 pp. 118.

Doucette WJ; (2000) Handbook of Property Estimation Methods for Chemicals. Boethling RS, Mackay D, eds. Boca Raton, FL: Lewis Publ pp. 141-188.

Franke, C., G. Studinger, G. Berger, S. Böhling, U. Bruckmann, D. Cohors-Fressenborg and U. Jöhncke (1994) The assessment of bioaccumulation. Chemosphere 29: 1501-14.

Hansch, C., Leo, A., and Hoekman, D. (1995) Exploring QSAR. Hydrophobic, Electronic, and Steric Constants. ACS Prof Ref Book. Heller SR, consult. ed. ,Washington, DC: American Chemical Society, p. 5.

Hansch, C., Leo, A., and Hoekman, D. (1995) Exploring QSAR. Hydrophobic, Electronic, and Steric Constants. ACS Prof Ref Book. Heller SR, consult. ed. ,Washington, DC: American Chemical Society, p. 5.

Lyman, WJ, WF Reehl, and DH Rosenblatt (1990) Handbook of Chemical Property Estimation Methods. Washington, DC: American Chemical Society pp. 4-9, 8-12, 15-1 to $15-29$. 
Meylan, WM; Howard, PH; and Boethling, RS (1999) Improved method for estimating bioconcentration/ bioaccumulation factor from octanol/water partition coefficient. Environ. Toxicol. Chem. 18: 664-672.

Perrin DD; (1965) Dissociation constants of organic bases in aqueous solution. IUPAC Chem Data Ser, Buttersworth, London.

Rao PSC, Davidson JM; (1982) Retention and Transformation of Selected Pesticides and Phosphorus in Soil-Water Systems, A Critical Review. Washington, DC: USEPA-60o/S382-06o.

Smith AE, Aubin AJ; (1992) Breakdown of Wldimethylamine in soils. J Agric Food Chem 40: 2299-2301.

Swann, R.L., Laskowski, D.A., McCall, P.J., van der Kuy, K. and Dishburger, H.J. (1983) A rapid method for estimation of the environmental parameters octanol/water partition coefficient, soil sorption constant water to air ratio and water solubility. Residue Rev. 85, pp. 17-28.

Von Oepen, B., Kordel, W., and Klein, W. (1991). "Sorption of nonpolar and polar compounds to soils: Processes, measurements and experience with the applicability of the modified OECD-guideline," Chemosphere 22, 285-304. 


\title{
Formaldehyde 50-oo-o
}

\section{ENVIRONMENTAL TRANSPORT, DISTRIBUTION, AND TRANSFORMATION}

\begin{abstract}
Air: According to a model of gas/particle partitioning of semivolatile organic compounds in the atmosphere (Bidleman 1988), formaldehyde, which has an estimated vapor pressure of 3,890 $\mathrm{mm} \mathrm{Hg}$ at 25 deg C (Boublik et al 1984), will exist in the gas phase in the ambient atmosphere (SRC). Gas-phase formaldehyde is degraded in the atmosphere by reaction with photochemically-produced hydroxyl radicals (SRC); the half-life for this reaction in air is $41 \mathrm{hrs}$ (SRC), calculated from its rate constant of $9.4 \mathrm{X}_{10} \mathrm{O}^{-12} \mathrm{cu}$ $\mathrm{cm} /$ molecule-sec at $25 \mathrm{deg} \mathrm{C}$ (Kwok and Atkinson 1994). The hydroxy radical initiated oxidation of formaldehyde also occurs in cloud droplets to form formic acid, a component of acid rain (Kwok and Atkinson 1994). Formaldehyde absorbs ultraviolet radiation at wavelengths of $>360 \mathrm{~nm}$ (Su et al 19795); therefore, formaldehyde may directly photolyze in sunlight (SRC). Formaldehyde has a half-life of $6 \mathrm{hrs}$ in simulated sunlight (Su et al 1979). The predicted half-life of formaldehyde due to photolysis in the lower atmosphere is $1.6 \mathrm{hrs}$ at a solar zenith of 40 degrees (Calvert et al 1972). Formaldehyde reacts with the $\mathrm{NO} 3$ radical by $\mathrm{H}$-atom abstraction with a half-life of 12 days (assuming an average $\mathrm{NO}_{3}$ radical concentration of $2 \mathrm{X}_{10} 9 / \mathrm{cu} \mathrm{cm}$ ) (Atkinson et al 1984).
\end{abstract}

Water: An estimated Koc value of 37 (SRC) determined from a log Kow of 0.35 (Hansch et al 1995) and a regression-derived equation (Lyman et al 1990), indicates that formaldehyde is not expected to adsorb to suspended solids and sediment (Swann et al 1983). Volatilization from water surfaces is not expected (Lyman et al 1990) based upon a Henry's Law constant of 3.4X10-7 atm-cu m/mole (Betterton and Hoffmann 1988). According to a classification scheme (Franke et al 1994), an estimated BCF of 3 (SRC), from its log Kow (Hansch et al 1995) and a regression-derived equation (Meylan et al 1999), suggests the potential for bioconcentration in aquatic organisms is low (SRC). Formaldehyde readily biodegrades under both aerobic and anaerobic conditions in the environment (SRC). Formaldehyde in aqueous effluent was degraded by activated sludge and sewage in 48-72 hr. In a die-away test using water from a stagnant lake, degradation was complete in 30 and $40 \mathrm{~h}$ under aerobic and anaerobic conditions, respectively (Kitchens et al 1976).

Soil/Sediment: Based on a classification scheme (Swann et al 1983), an estimated Koc value of 37 (SRC), determined from a log Kow of 0.35 (Hansch et al 1995) and a regression-derived equation (Lyman et al 1990), indicates that formaldehyde is expected to have very high mobility in soil (SRC). Volatilization of formaldehyde from moist soil surfaces is not expected to be an important fate process (SRC) given a Henry's Law 
constant of 3.4X10-7 atm-cu m/mole (Betterton and Hoffmann 1988). Volatilization of formaldehyde from dry soil surfaces will occur because it is a gas (Boublik et al 1984). Formaldehyde readily biodegrades under both aqueous aerobic and anaerobic conditions in the environment which suggests that these processes may be important in soil (SRC). Formaldehyde in aqueous effluent was degraded by activated sludge and sewage in 48-72 $\mathrm{hr}$. In a die-away test using water from a stagnant lake, degradation was complete in 30 and $40 \mathrm{hrs}$ under aerobic and anaerobic conditions, respectively (Kitchens et al 1976).

Biota: An estimated BCF of 3 was calculated for formaldehyde (SRC), using a log Kow of 0.35 (Hansch et al 1995) and a regression-derived equation (Meylan et al 1999). According to a classification scheme (Franke et al 1994), this BCF suggests the potential for bioconcentration in aquatic organisms is low (SRC). Experiments performed on a variety of fish and shrimp show no bioconcentration of formaldehyde (Hose and Lightner 1980, Sills and, Allen 1979).

\section{Regulations}

MCL (Drinking Water): $0.7 \mathrm{ug} / \mathrm{L}$

Universal Treatment Standards (UTS): none

Groundwater Water Quality Criterion : $100 \mathrm{ug} / \mathrm{L}$

PQL: $5.0 \mu \mathrm{g} / \mathrm{g}$

IDLH: $20 \mathrm{ppm}$

EPA RfD: $0.2 \mathrm{mg} / \mathrm{kg}$-day

EPA RfC: $0.0098 \mathrm{mg} / \mathrm{m}^{3}$

PRG: residential soil $\mathrm{mg} / \mathrm{kg}$ industrial soil $\mathrm{mg} / \mathrm{kg}$ ambient air $\mathrm{ug} / \mathrm{m} \quad$ tap water $\mathrm{ug} / \mathrm{L}$ 9200 100000 0.15 5500

\section{Remediation}

Use fluorocarbon water spray, Cellosize and Hycar to diminish vapors. Sodium carbonate, ammonium hydroxide, or sodium sulfite can neutralize the spill (Prager 1995). 


\section{References}

Atkinson R, Plum CN, Carter WPL, Winer AM, and Pitts JN. (1984) Rate Constants for the gas-phase reactions of nitrate radicals with a series of organics in air at $298 \pm 1$. J Phys Chem 88: $1210-5$.

Betterton EA, Hoffmann MR; (1988) Henry's Law Determinations for Some Environmentally Important Aldehydes. Environ Sci Technol 22: 1415-8.

Bidleman, T.F. (1988) Atmospheric processes: Great Lakes Pesticide Report Environmental Science and Technology. 22(4):361-367.

Boublik T, Fried V, Hala E. (1984). The vapor pressures of pure substances. Vol. 17. Amsterdam, Netherlands: Elsevier Sci Publ p. 44.

J. G. Calvert, J. A. Kerr, K. L. Demerjian, and R. D. McQuigg (1972) Photolysis of formaldehyde as a hydrogen atom source in the lower atmosphere. Science 175: 751-52.

Chameides WL, Davis DD (1983) Aqueous source of formic acid in clouds. Nature 304: 427-9.

CITI; Biodegradation and Bioaccumulation Data of Existing Chemicals. Formaldehyde (50-0o-0). Available from the Database Query page at http://www.safe.nite.go.jp/english/kizon/KIZON_start_hazkizon.html.

Franke, C., G. Studinger, G. Berger, S. Böhling, U. Bruckmann, D. Cohors-Fressenborg and U. Jöhncke (1994) The assessment of bioaccumulation. Chemosphere 29: 1501-14.

Hansch C, Leo, A., and Hoekman, D. (1995) Exploring QSAR. Hydrophobic, Electronic, and Steric Constants. ACS Prof Ref Book. Hellers SR, consult. ed., Washington, DC: Amer Chem Soc p. 3.

Hose JE, Lightner DV (1980) Absence of formaldehyde residues in penaeid shrimp exposed to formalin. Aquaculture 21: 197-201.

Kitchens, J.F., Casner, R.E., Edwards, G.S., Howard III, W.E. and Macri, B.J. (1976) Investigation of selected potential environmental contaminants; formaldehyde. Washington DC: USEPA, Off Tox Subst USEPA 560/2-76-009 p. 99-110. 
Kwok ESC, Atkinson R; (1994) Estimation of hydroxyl radical reaction rate constants for gas-phase organic compounds using a structure-reactivity relationship: an update. Riverside, CA: Univ CA, Statewide Air Pollut Res Ctr. CMA Contract No. ARC-8.o-OR.

Lyman, WJ, WF Reehl, and DH Rosenblatt (1990) Handbook of Chemical Property Estimation Methods. Washington, DC: Amer Chem Soc pp. 4-9, 15-1 to 15-29.

Meylan, WM, Howard, PH, Boethling, RS, Aronson, D, Printup, H, Gouchie, S. (1999) Improved method for estimating bioconcentration/bioaccumulation factor from octanol/water partition coefficient. Environ Toxicol Chem 18(4):664-72.

Prager, J.C. (1995) Environmental Contaminant Reference Databook Volume 1. New York, NY: Van Nostrand Reinhold, p. 707.

Sills JB, Allen JL. (1979). Residues of formaldehyde undetected in fish exposed to formalin. Prog Fish Cult 41:67-68.

Su F, Calvert G, Shaw JH. (1979). Mechanism of the photooxidation of gaseous formaldehyde. J Phys Chem 83:3185-3191.

Swann, R.L., Laskowski, D.A., McCall, P.J., van der Kuy, K., and Dishburger, H.J. (1983) A rapid method for estimation of the environmental parameters octanol/water partition coefficient, soil sorption constant. water to air ratio and water solubility. Residue Rev. 85, pp. 17-28. (http://toxnet.nlm.nih.gov/cgi-bin/sis/search/r?dbs+hsdb:@term+@rn+6o34-4). 


\section{Hydrazine 302-01-2}

\section{ENVIRONMENTAL TRANSPORT, DISTRIBUTION, AND TRANSFORMATION}

Air: Hydrazine and dimethylhydrazines degrade rapidly in air through reactions with ozone, hydroxyl $(\mathrm{OH})$ radicals, and nitrogen dioxide (WHO 1987). The reaction of hydrazine and 1,1- dimethylhydrazine with ozone is probably the major fate of these chemicals in the atmosphere. The reaction rate constant for hydrazine, derived from its decay rate in the presence of excess ozone, was about $3 \mathrm{X}^{-17} \mathrm{~cm}^{3}$ molecule-s and for 1,1dimethylhydrazine the rate was greater than $1 \mathrm{X}^{-15} \mathrm{~cm}^{3}$ molecule-s (Atkinson and Carter 1984). Major reaction products were hydrogen peroxide for the hydrazine reaction and dimethylnitrosamine (about 60\%) for the 1,1-dimethylhydrazine reaction. Estimated atmospheric half-lives ranged from less than 10 minutes for hydrazine during an ozone pollution episode to less than 2 hours under usual conditions, with a half-life about onetenth that time for 1,1-dimethylhydrazine (Tuazon et al. 1981). Reported results of additional studies indicate a reaction rate constant for hydrazine of $2.5 \times 10^{-16} \mathrm{~cm}^{3}$ molecule-s, resulting in an estimated half-life of less than 1 min (Stone 1989). The reported measured rate constant for reaction of hydrazine with atmospheric hydroxyl $(\mathrm{OH})$ radicals producing ammonia and nitrogen gas was $6.1 \times 10^{-11} \mathrm{~cm}^{3}$ molecule-s (Harris et al. 1979). The rate constant for 1,1-dimethylhydrazine was not measured since the chemical decomposed rapidly in the test system, but the value was estimated at $5 \mathrm{X}_{10} \mathrm{O}^{-11}$ $\mathrm{cm}^{3}$ molecule-s. Assuming an average $\mathrm{OH}$ radical concentration of about 106 molecule $/ \mathrm{cm}^{3}$, the tropospheric half-lives of both chemicals due to reaction with $\mathrm{OH}$ were estimated to be about 3 hours. The half-lives are expected to range from less than 1 hour in polluted urban air to 3-6 hours in less polluted atmospheres (Tuazon et al. 1981). Hydrazine and 1,1-dimethylhydrazine react rapidly with nitrogen oxides in both the light and dark, with a half-life of about 2 hours for hydrazine and less than 10 minutes for 1,1dimethylhydrazine (Pitts et al. 1980). Hydrazine and 1,1-dimethylhydrazine may also be removed from the atmosphere by autoxidation. In a dark reaction chamber, the approximate half-lives of hydrazine ranged from 1.8 to 5 hours, with the lower value measured at higher humidity. Reported values for 1, 1 dimethylhydrazine under similar conditions were 5.9-9 hours. Surface interactions are important in controlling the rates of these reactions (Stone 1989). Although data were not located for 1,2-dimethylhydrazine, this chemical is expected to be degraded in the atmosphere by undergoing the same reactions as hydrazine and 1,1-dimethylhydrazine, although the rate and extent of degradation may be different.

Water: Hydrazine and 1,1-dimethylhydrazine degrade in aqueous systems, but the rate of degradation is dependent on specific aquatic environmental factors, including $\mathrm{pH}$, 
hardness, temperature, oxygen concentration, and the presence of organic matter and metal ions (Moliner and Street 1989 and WHO 1987). Oxidation and biodegradation are the primary removal mechanisms. Reaction of hydrazine with dissolved oxygen is catalyzed by metal ions, particularly copper (EPA 1984). The reaction rate is strongly influenced by $\mathrm{pH}$; degradation proceeds more rapidly in alkaline solutions. Hydrazine is rapidly removed from polluted waters, with less than one-third of the original concentration remaining in dirty river water after 2 hours (Slonim and Gisclard 1976). More than $90 \%$ of the hydrazine added to pond or chlorinated, filtered county water disappeared after 1 day. However, chlorinated, filtered, and softened city water contained almost the original amount of hydrazine after 4 days. Organic matter in the water and hardness were reported to be the major factors in the differing rates of degradation.

The primary reaction pathway for hydrazine degradation in water produces nitrogen gas and water (Moliner and Street 1989). In oxygen-deficient waters or in the presence of metal ions which serve as catalysts, ammonia may also be produced. The reaction of 1,1dimethylhydrazine with dissolved oxygen in water may proceed by a process atalyzed by copper ions or by an uncatalyzed reaction (Banerjee et al. 1984). The products include dimethylnitrosamine, formaldehyde, dimethylamine, and other related chemicals. Dimethylnitrosamine did not form in dilute solutions, which might be encountered in ambient waters, but was reported in concentrated solutions, which could be present in the vicinity of spills (EPA 1984). The reported half-life of 1,1-dimethylhydrazine in ponds and seawaters ranged from 10 to 14 days, presumably because of reaction with oxygen and other free radicals (EPA 1984). Biodegradation may be a significant removal process at low hydrazine concentrations in ambient waters, but at higher concentrations the chemical is toxic to microorganisms. In the presence of bacterial cells, more than $90 \%$ of the hydrazine was degraded in six water samples containing $11 \mu \mathrm{g} / \mathrm{mL}$ of the chemical within 2 hours (Ou and Street 1987). Lower degradation rates were reported with increasing hydrazine concentrations. No degradation was reported for incubation of these waters without bacteria. Additional studies indicate that hydrazine and 1,1dimethylhydrazine are toxic to bacterial populations. Concentrations of hydrazine and 1,1-dimethylhydrazine that reduced bacterial metabolism by 50\% ranged from 14.6 to 145 $\mathrm{mg} / \mathrm{L}$ and from 19.2 to 9,060 mg/L, respectively (Kane and Williamson 1983). Thus, biological treatment would not be useful for spills of these chemicals into the aquatic environment.

Sediment and Soil: Hydrazine appears to degrade more rapidly in soil than in water, with oxidation and biodegradation as the main removal processes. Hydrazine applied to nonsterile Arredondo soil (fine sand) at concentrations of 10, 100, and $500 \mu \mathrm{g} / \mathrm{g}$ was completely degraded in 1.5 hours, 1 day, and 8 days, respectively (Ou and Street 1987). In this study, comparison to degradation rates in sterile soils indicated that autoxidation 
appeared to be the major factor contributing to disappearance of the chemical, but the study authors attributed about $20 \%$ of removal to biodegradation. Several heterotrophic soil bacteria were reported to degrade hydrazine, indicating that microbial degradation may contribute to removal of the chemical from soil (Ou 1987).

Biota: In a study of hydrazine in aqueous systems, the chemical was reported to be absorbed by guppies from a $0.5 \mu \mathrm{g} / \mathrm{L}$ solution (Slonim and Gisclard 1976). After 96 hours, the hydrazine concentration in fish was $144 \mu \mathrm{g} / \mathrm{g}$, indicating a moderate tendency to bioconcentrate. However, the bioconcentration of hydrazine and dimethylhydrazines is not expected to be important in aquatic systems because of the rapid degradation of these chemicals in water as well as their low octanol-water partition coefficients.

Environmental partitioning: Hydrazine or dimethylhydrazines released to water or soil may volatilize into air or sorb onto soil. These chemicals have low vapor pressures and are miscible in water. Therefore, volatilization is not expected to be an important removal process. Reported evaporation rates from aqueous solutions under laboratory conditions were $0.49 \mathrm{mg} / \mathrm{cm}^{2}$ minute for hydrazine and $13 \mathrm{mg} / \mathrm{cm}^{2}$ minute for 1,1dimethylhydrazine (EPA 1984a). The significance of these values to environmental conditions is unknown. Data from other studies indicate that volatilization of these chemicals from water increases with higher concentrations of the chemical and in the presence of sunlight (due to increased temperature of the hydrazine pool). Based on air dispersion modeling, volatilization of hydrazine from surface soil following a spill is expected to be sufficient (16-100 $\mathrm{mg} / \mathrm{cm}^{2}$ hour) to generate a short-term ambient air concentration of $4 \mathrm{mg} / \mathrm{m}^{3}$ up to $2 \mathrm{~km}$ downwind of the spill under worst-case meteorological conditions (MacNaughton et al. 1981). Degradation of hydrazine would likely reduce the concentration within several hours. Atmospheric transport of hydrazine or dimethylhydrazines may occur, but transport will be limited by the high reactivity of the chemicals in the atmosphere. No data were located on deposition of hydrazine or dimethylhydrazines from air to water or soil, but deposition would also be limited by their high reactivity. Hydrazine undergoes complex interactions with soils, including both reversible physical-sorption and irreversible chemisorption to colloids (Mansell et al. 1988). In a study on the adsorption and leaching characteristics of hydrazine fuels, no adsorption of 1,1-dimethylhydrazine was observed on sand, with almost $100 \%$ of the chemical leaching with water (Braun and Zirrolli 1983). In three other soils, adsorption ranged from $26 \%$ to $80 \%$. No correlation between adsorption and soil organic content or $\mathrm{pH}$ was observed. The mechanisms of attenuation in soil materials were not reported. However, reported results of additional hydrazine adsorption studies with clays and soils indicate that adsorption may be correlated with soil organic matter and clay content and is highly dependent on $\mathrm{pH}$; hydrazine appears to be adsorbed by different mechanisms under acidic and alkaline conditions (Moliner and Street 1989). 


\section{Regulatory}

UTS: None established

IDLH: $50 \mathrm{ppm}$

EPA Rf D: None established

EPA RfC: None established

PRG: residential soil $\mathrm{mg} / \mathrm{kg}$ industrial soil $\mathrm{mg} / \mathrm{kg}$ ambient air $\mathrm{ug} / \mathrm{m} \quad$ tap water $\mathrm{ug} / \mathrm{L}$ $\begin{array}{llll}0.16 & 0.82 & 0.00039 & 0.022\end{array}$

\section{Remediation}

Hydrazine has been disposed of by dilution with water to form at least a $400 \mathrm{~g} / \mathrm{liter}$ solution, followed by neutralization with dilute sulfuric acid and drainage into a sewer with abundant water (IRPTC, 1985). However, it should be noted that even very dilute solutions of $0.1 \mathrm{mg} /$ liter can be toxic for aquatic life. Alternatively, hydrazine has been burnt in an open pit after the addition of a hydrocarbon solvent (IRPTC, 1985). A better procedure is to dilute with abundant water and then oxidize the diluted solution (to below $20 \mathrm{~g}$ /liter) with hydrogen peroxide, calcium hypochlorite, orsodium hypochlorite before draining into a sewer (NEPSS, 1975).

Hydrazine vapor emissions can be controlled by scrubbing, using water as the scrubbing liquid, or by the direct flame of catalytic incineration (Gordon \& Lewandowski, 1980). Hydrazine sulfate, a commonly-used derivative, may be disposed of by incineration (IRPTC, 1985). Hydrazine degradation is very rapid in the environment.

\section{References}

Atkinson R, Carter WP. (1984). Kinetics and mechanisms of the gas-phase reactions of ozone withorganic compounds under atmospheric conditions. Chem Rev 84:437-470.

Banerjee S, Pack EJ, Sikka H. (1984). Kinetics of oxidation of methylhydrazines in water, factors controlling the formation of 1,l-dimethylnitrosamine. Chemosphere 4:549-559.

Braun BA, Zirrolli JA. (1983). Environmental fate of hydrazine fuels in aqueous and soil environments. Air Force Engineering and Services Center, Engineering and Services Laboratory, Tyndall Air Force Base, FL. ESL-TR-82-45. 
EPA. (1984). Health and environmental effects profile for 1,1-dimethylhydrazine. Cincinnati, OH: U.S. Environmental Protection Agency, Office of Research and Development. EPA-600/X-84-134.

Gordon, A. and Lewandowski, P.E. (1980) Control of vapor emissions from hydrazine-based fuels, Newark, New Jersey, Institute of Technology, Department of Chemical Engineering (AFOSR-TR80-053).

Harris GW, Atkinson R, Pitts, JN. (1979). Kinetics of the reactions of the OH radical with hydrazine and methylhydrazine. J Phys Chem 83:2557-2559.

IRPTC (1985) Treatment and disposal methods for waste chemicals, Geneva, International Register of Potentially Toxic Chemicals, United Nations Environment Programme, pp. 188-189.

Kane DA, Williamson KJ. (1983). Bacterial toxicity and metabolism of hydrazine fuels. Arch Environ Contam Toxicol 12:447-453.

MacNaughton MG, Stauffer TB, Stone DA. (1981). Environmental chemistry and management of hydrazine. Aviation Space Environ Med 149-153.

Mansell RS, Bloom SA, Downs WC. (1988). Numerical simulation of hydrazine transport in a sandy soil. In: The Third Conference on the Environmental Chemistry of Hydrazine Fuels. ESL-TR-87-74, 177-189.

Moliner AM, Street JJ. (1989). Decomposition of hydrazine in aqueous solutions. J Environ Qua1 18:483-487.

NEPSS (1975) Disposal of wastewater containing hydrazine. In: Pollution solution, California, Naval Environmental Protection Support Service, Navy Environmental Support Office.

Ou LT. (1987). Microbial degradation of hydrazine. Bull Environ Contam Toxicol 39:7885 .

Ou LT, Street JJ. (1987). Hydrazine degradation and its effect on microbial activity in soil. Bull Environ Contam Toxicol 38:179-183.

Pitts JN, Tuazon EC, Carter WP.. (1980). Atmospheric chemistry of hydrazines: Gas phase kinetics and mechanistic studies. ESL-TR-80-39.

Slonin AR, Gisclard JB. (1976). Hydrazine degradation in aquatic systems. Bull Environ Contam Toxicol 16:301-309. 
Schmidt EW. (1988). One hundred years of hydrazine chemistry. In: The Third Conference on the Environmental Chemistry of Hydrazine Fuels. ESL-TR-87-74, 4-16.

Stone DA. (1989). Atmospheric chemistry of propellant vapors. Toxicol Lett 49:349-360.

Tuazon EC, Carter WP, Winer AM. (1981). Reactions of hydrazines with ozone under simulated atmospheric conditions. Environ Sci Technol 15:823-828.

WHO. (1987). Environmental health criteria 68: Hydrazine. World Health Organization, Geneva, Switzerland, 1-89. 


\section{Hydrogen Chloride (Hydrochloric Acid) 7647-01-o}

\section{ENVIRONMENTAL TRANSPORT, DISTRIBUTION, AND TRANSFORMATION}

Air: Hydrogen chloride is removed from air by wet deposition as chloride salts with an atmospheric lifetime of 1-5 days (Kao 1994).

Water: If released to water, hydrogen chloride dissociates readily in water to chloride and hydronium ions, decreasing the $\mathrm{pH}$ of the water (ATSDR 2002). A Henry's law constant of $2.04 \mathrm{X}_{10} \mathrm{O}^{6} \mathrm{~mol} / \mathrm{L}$ atm $\left(4.90 \mathrm{X} 10^{-10} \mathrm{cu} \mathrm{m}\right.$ atm $\left./ \mathrm{mol}\right)$ has been reported for hydrochloric acid (Brimblecombe and Clegg 1989). This Henry's Law constant indicates that hydrochloric acid is expected to be essentially nonvolatile from water surfaces (Lyman et al 1990).

Soil/Sediment: If released to soil, hydrogen chloride will evaporate from dry soil surfaces and dissociate into chloride and hydronium ions in moist soil (ATSDR 2002).

\section{Regulations}

MCL (Drinking Water): None (completely disassociates)

Groundwater Water Quality Criterion: $0.2 \mathrm{ug} / \mathrm{L}$

IDLH: $50 \mathrm{ppm}$

EPA RfD: $0.0057 \mathrm{mg} / \mathrm{kg}$-day

EPA RfC: $0.02 \mathrm{mg} / \mathrm{m}^{3}$

PRG: residential soil $\mathrm{mg} / \mathrm{kg}$ industrial soil $\mathrm{mg} / \mathrm{kg}$ ambient air $\mathrm{ug} / \mathrm{m} \quad$ tap water $\mathrm{ug} / \mathrm{L}$ 21

\section{Remediation}

Land spill: Dig a pit, pond, lagoon, holding area to contain liquid or solid material. If time permits, pits, ponds, lagoons, soak holes, or holding areas should be sealed with an impermeable flexible membrane liner. Dike surface flow using soil, sand bags, foamed polyurethan or foamed concrete. Absorb bulk liquid with fly ash or cement powder. Neutralize with agricultural lime $(\mathrm{CaO})$, crushed limestone $\left(\mathrm{CaCO}_{3}\right)$ or sodium 
bicarbonate $\left(\mathrm{NaHCO}_{3}\right)$. Water spill: Neutralize with agricultural lime $(\mathrm{CaO})$, crushed limestone $\left(\mathrm{CaCO}_{3}\right)$ or sodium bicarbonate $\left(\mathrm{NaHCO}_{3}\right)$.

Air release: Apply water spray or mist to knock down vapors. Vapor knockdown water is corrosive or toxic and should be diked for containment (Association of American Railroads).

\section{References}

Association of American Railroads; Bureau of Explosives. Emergency Handling of Hazardous Materials in Surface Transportation. Association of American Railroads, Pueblo, CO. 2005, p. 475.

ATSDR; ToxFAQs for Hydrogen Chloride, April 2002, available at: http://www.atsdr.cdc.gov/tfacts173.html.

Brimblecombe, P. and Clegg, S. L.: (1985) Erratum, J. Atmos. Chem., 8, 95.

Kao, A.S. (1994) Formation and removal reactions of hazardous air pollutants, J Air Waste. Manage Assoc 44, 683-96.

Lyman, WJ, WF Reehl, and DH Rosenblatt (1990) Handbook of Chemical Property Estimation Methods. Washington, DC: American Chemical Society pp. 15-1 to 15-29. 


\title{
Hydrogen Cyanide $\quad 74-90-8$
}

\section{ENVIRONMENTAL TRANSPORT, DISTRIBUTION, AND TRANSFORMATION}

\begin{abstract}
Air: Most cyanide in the atmosphere exists almost entirely as hydrogen cyanide gas, although small amounts of metal cyanides may be present as particulate matter in the air (EPA 1984). Hydrogen cyanide is very resistant to photolysis at wavelengths of normal sunlight (EPA 1979). The most important reaction of hydrogen cyanide in air is the reaction with photochemicallygenerated hydroxyl radicals and subsequent rapid oxidation to carbon monoxide (CO) and nitric oxide (NO); photolysis and reaction with ozone are not important transformation processes, and reaction with singlet oxygen $(\mathrm{O} 1 \mathrm{D})$ is not a significant transformation process except at stratospheric altitudes where singlet oxygen is present in significant concentrations (Cicerone and Zellner 1983). The rate of hydroxyl radical reaction with hydrogen cyanide in the atmosphere depends on the altitude, and the rate of the reaction is at least an order of magnitude faster at lower tropospheric altitudes $(0-8 \mathrm{~km})$ than at upper tropospheric altitudes $(10-12 \mathrm{~km})($ Cicerone and Zellner 1983). Based on a reaction rate constant of $3 \times 10^{-14} \mathrm{~cm}^{3} /\left(\right.$ molecule-sec) at $25^{\circ} \mathrm{C}$ (Fritz et al. 1982 ) and assuming an average hydroxyl radical concentration of $5 \times 10^{5}$ molecules $/ \mathrm{cm}^{3}$, the residence time for the reaction of hydrogen cyanide vapor with hydroxyl radicals in the atmosphere is $\approx 2$ years. This value compares well with the atmospheric residence time derived by Cicerone and Zellner (1983) of approximately 2.5 years, with a range of 1.3-5.0 years, depending on the hydroxyl radical concentrations assumed. Using the equation $\mathrm{t}^{1} / 2=0.693 \tau$ for converting residence time $(\tau)$ to half-life ( $\left.t \frac{1}{2}\right)$ (Lyman 1982) and an estimated atmospheric residence time for hydrogen cyanide of 2-3 years, and assuming first-order kinetics for the reaction of hydrogen cyanide with hydroxyl radicals, an atmospheric half-life of 1.4- 2.9 years can be calculated for hydrogen cyanide.
\end{abstract}

Cyanogen is reactive and does not persist in the environment unchanged (EPA 1978). Cyanogen reacts slowly with water to yield hydrogen cyanide and cyanic acid (HOCN) among other products (EPA 1979) and this hydrolysis reaction may be a possible atmospheric degradation pathway. Cyanogen has also been shown to react with hydroxyl radicals in the gas phase (Atkinson 1989). Based on a rate constant of $2.5 \times 10^{-15} \mathrm{~cm}^{3} /\left(\right.$ molecule-sec) at $27^{\circ} \mathrm{C}$ and assuming an average hydroxyl radical concentration of $5 \times 10^{5}$ molecules $/ \mathrm{cm}^{3}$, the residence time for the reaction of hydrogen cyanide vapor with hydroxyl radicals in the atmosphere is $\approx 25$ years. Therefore, the reaction of cyanogen with photochemically-induced hydroxyl radicals will not play a significant role in the degradation of this compound in air.

No specific information was found in the available literature on the transformation and degradation of cyanogen chloride or thiocyanates in air. However, cyanogen chloride has been shown to undergo slow hydrolysis in neutral aqueous solution (rate constant at $\mathrm{pH} 7$ of $6.45 \times 10^{-5}$ $\mathrm{mol}^{-1} \mathrm{sec}^{-1}$ ) (U.S. Army 1989). Therefore, hydrolysis of this compound may be a possible atmospheric degradation pathway in air.

Water: Cyanide occurs most commonly as hydrogen cyanide in water, although it can also occur as the cyanide ion, alkali and alkaline earth metal cyanides (potassium cyanide, sodium cyanide, calcium cyanide), relatively stable metallocyanide complexes (ferricyanide complex $\left[\mathrm{Fe}(\mathrm{CN})_{6}\right]^{-3}$ ), moderately stable metallocyanide complexes (complex nickel and copper cyanide), or easily decomposable metallocyanide complexes (zinc cyanide $\left[\mathrm{Zn}(\mathrm{CN})_{2}\right]$, cadmium cyanide $\left.\left[\mathrm{Cd}(\mathrm{CN})_{2}\right]\right)$. The environmental fate of these cyanide compounds varies widely (EPA 1979).

In water, hydrogen cyanide and cyanide ion exist in equilibrium with their relative concentrations primarily dependent on $\mathrm{pH}$ and temperature. At $\mathrm{pH}<8,>93 \%$ of the free cyanide in water will exist as undissociated hydrogen cyanide (EPA 1978). Hydrogen cyanide can be hydrolyzed to formamide, which is subsequently hydrolyzed to ammonium and formate ions (EPA 1979). 
However, the relatively slow rates of hydrolysis reported for hydrogen cyanide in acidic solution (Krieble and McNally 1929; Krieble and Peiker 1933) and of cyanides under alkaline conditions (Wiegand and Tremelling 1972) indicate that hydrolysis is not competitive with volatilization and biodegradation for removal of free cyanide from ambient waters (EPA 1979).

The significance of photolysis in the fate of cyanides in water has not been fully investigated. Hydrogen cyanide and cyanide ions in aqueous solution have been found to be very resistant to photolysis by natural sunlight, except under heterogeneous photocatalytic conditions (EPA 1979; Frank and Bard 1977). Photocatalytic oxidation may not be significant in natural waters, however, because of significant light reduction at increasingly greater depths (EPA 1992). In clear water or at water surfaces, some metallocyanides, such as ferrocyanides and ferricyanides, may decompose to the cyanide ion by photodissociation and subsequently form hydrogen cyanide. For example, diurnal changes in free cyanide concentrations in the drainage from spent precious metal ore heaps were found to maximize around mid-day due to the photodissociation of iron and cobalt cyanocomplexes (Johnson et al. 2002). Because of adsorption of ferrocyanide onto soil surfaces and sediment of surface waters, and light scattering in turbid waters in the field, the rate of free cyanide formation from the photolysis of ferrocyanide in runoff and surface water from washout of ferrocyanide in de-icing salt will be slower than from laboratory photolysis with clean water (EPA 1979).

Sediment and Soil: Analogous to the fate of cyanides in water, it is predicted that the fate of cyanides in soil would be dependent on cyanide concentrations, $\mathrm{pH}$, temperature, metal content, concentration of microbes, availability of nutrients, and acclimation of microbes. Cyanide may occur as hydrogen cyanide, alkali metal salts, or as immobile metallocyanide complexes. In soil, cyanide present at low concentrations would biodegrade under aerobic conditions with the initial formation of ammonia, which would be converted to nitrite and nitrate in the presence of nitrifying bacteria. Under anaerobic conditions, the cyanides ion will denitrify to gaseous nitrogen (Richards and Shieh 1989). Upper limits of 200 and $2 \mathrm{ppm}(\mathrm{mg} / \mathrm{kg} \mathrm{CN}-)$ have been reported for uninhibited aerobic and anaerobic biodegradation of cyanide in soil respectively (Fueller 1985); however, these limits have not been confirmed in other studies (Thomas and Lester 1993). Cyanide ions in soil are not involved in oxidation-reduction reactions but may undergo complexation reactions with metal ions in soil (EPA 1978).

Biota: There are no data available to indicate that simple metal cyanides and hydrogen cyanide bioconcentrate in aquatic organisms (EPA 1979, 1985, 1992). Bioconcentration factors (BCFs) of 0.73 and 1.62 can be calculated for hydrogen cyanide, using the equation of Veith et al. (1979) for the BCF of a chemical in whole fish ( $\log \mathrm{BCF}, 0.85$; $\log$ Kow, -0.70) and the log Kow values. Similarly, the calculated BCF for sodium cyanide is 0.47 . There is some evidence that certain metal cyanide complexes bioaccumulate in aquatic organisms. Fish from water with soluble silver and copper cyanide complexes were found to have metal cyanides in their tissues at concentrations ranging up to 168 and $304 \mu \mathrm{g} / \mathrm{g}$, respectively (wet or dry weight not specified) (EPA 1979). It is difficult to evaluate the toxicologic significance of bioaccumulation of metal cyanide complexes because these compounds are much less toxic than soluble hydrogen cyanide, sodium cyanide, or potassium cyanide (EPA 1992). There is no evidence of biomagnification of cyanides in the food chain (EPA 1978). Accumulation of cyanide in food webs is not expected, considering the rapid detoxification of cyanide by most species and the lethal effects of large doses of cyanide (EPA 1978).

Environmental Partitioning: Because hydrogen cyanide is a gas and has a relatively slow degradation rate in air, the atmosphere will be the ultimate sink for this compound. Almost all of 
the hydrogen cyanide released to the atmosphere remains in the lower altitudes (troposphere); only $2 \%$ of tropospheric hydrogen cyanide is transferred to the stratosphere (Cicerone and Zellner 1983). Cyanide has the potential to be transported over long distances from its emission source. Despite higher water solubility at saturated pressure, the removal of hydrogen cyanide by rainwater appears to be a negligible partitioning pathway (Cicerone and Zellner 1983). Because hydrogen cyanide is a gas, its removal from air by dry deposition is also likely to be negligible. However, metal cyanide particles, particularly water-soluble cyanide particles, are expected to be removed from the air by both wet and dry deposition.

Volatilization of hydrogen cyanide would be a significant loss mechanism for cyanides from soil surfaces at a $\mathrm{pH}<9.2$. Cyanides are fairly mobile in soil. Mobility is lowest in soils with low $\mathrm{pH}$ and high concentrations of free iron oxides, positively charged particles, and clays (e.g., chlorite, kaolin, gibbsite), and highest in soils with high $\mathrm{pH}$, high concentrations of free $\mathrm{CaCO} 3$ and negatively charged particles, and low clay content (EPA 1979). Although cyanide has a low soil sorption capability, it is usually not detected in groundwater, probably because of fixation by trace metals through complexation or transformation by soil microorganisms (EPA 1978). In soils, where cyanide levels are high enough to be toxic to microorganisms (i.e., landfills, spills), this compound may leach into groundwater (EPA 1984). Also, leaching of cyanide into a shallow aquifer can occur, as demonstrated by the high concentration of cyanide $(1,200 \mu \mathrm{g} / \mathrm{L})$ in groundwater sampled from the Biscayne Aquifer in Dade County, Florida, which lies below a solid waste site (Myers 1983).

\section{Regulatory}

Drinking Water Standards: MCLG: 0.2 mg/L (cyanide)

RfD: $0.02 \mathrm{mg} / \mathrm{kg} /$ day

RfC: $0.003 \mathrm{mg} / \mathrm{m}^{3}$

UTS: None established

Soil PQL: 3 ug/L

IDLH: $25 \mathrm{mg} / \mathrm{m}^{3}$

PRG: residential soil $\mathrm{mg} / \mathrm{kg}$ industrial soil $\mathrm{mg} / \mathrm{kg} \quad$ ambient air $\mathrm{ug} / \mathrm{m} \quad$ tap water $\mathrm{ug} / \mathrm{L}$ 11 35

\section{Remediation}

Cyanides are not persistent in water or soil. Cyanides may accumulate in bottom sediments, but residues are generally as low as $<1 \mathrm{mg} / \mathrm{kg}$ even near polluting sources. Majority of an accidental release of cyanide is volatilized to the atmosphere where it is quickly diluted and degraded by ultra violet. Other factors, such as biological oxidation, precipitation and the effects of sunlight also contribute to cyanide degradation. There is 
no evidence of bioaccumulation in the food chain, and hence, secondary poisoning does not occur.

\section{References}

Cicerone RJ and Zellner R. (1983). The atmospheric chemistry of hydrogen cyanide (HCN). J Geophys Res 88:10689-10696.

EPA. (1978). Reviews of the environmental effects of pollutants. V. Cyanide. Cincinnati, OH: U.S. Environmental Protection Agency Health Effects Research Laboratory, Office of Research and Development. PB289920.

EPA. (1979). Cyanides. In: Water-related environmental fate of 129 priority pollutants. Vol. 1. Washington, DC: U.S. Environmental Protection Agency, Office of Water Planning and Standards, Office of Water and Waste Management. EPA440479029a. PB80204373. 12-1-12-12.

EPA. (1984). Health effects assessment for cyanide. Washington, DC: U.S. Environmental Protection Agency. EPA540186011.

EPA. (1985). Ambient water quality for cyanide - 1984. Washington, DC: Office of Water Regulations and Standards, Criteria and Standards Division. EPA440584028. PB85227460.

EPA. (1992). U.S. Environmental Protection Agency. Fed Regist 57:26248.

Fueller WH. (1985). Cyanides in the environment with particular attention to the soil. In: Van Zyl D, ed. Cyanide and the environment: Proceedings of a conference Tucson, Arizona December 11-14, 1984: Volume 1. Fort Collins, CO: Colorado State University, 1946.

Frank SN and Bard AJ. (1977). Heterogeneous photocatalyst oxidation of cyanide ion in aqueous solutions at titanium dioxide powder. J Amer Chem Soc 99(1):303-304.

Johnson CA, Leinz RW, and Grimes DJ. (2002). Photochemical changes in cyanide speciation in drainage from a precious metal ore heap. Environ Sci Technol 36:840-845.

Krieble VK and McNally JG. (1929). The hydrolysis of hydrogen cyanide by acids. J Am Chem Soc 51:3368-3375. 
Krieble VK and Peiker AL. (1933). The hydrolysis of hydrogen cyanide by acids II. J Am Chem Soc 55:2326-2331.

Lyman, W. (1982). Atmospheric residence time. In: Lyman WJ, Reehl WF, Rosenblatt DH, eds. Handbook of chemical property estimation methods: Environmental behavior of organic compounds. New York, NY: McGraw Hill Book Company, 10-2-10-33.

Myers VB. (1983). Remedial activities at the Miami Drum site, Florida. In: National conference on management of uncontrolled hazardous waste sites. Silver Springs, MD: Hazardous Materials Control Research Institute, 354-357.

Richards DJ and Shieh WK. (1989). Anoxic-oxic activated-sludge treatment of cyanides and phenols. Biotechnol Bioeng 33:32-38.

Thomas AO and Lester JN. (1993). The microbial remediation of former gasworks sites. A review. Environ Technol 14(1):1-24.

Wiegand GH and Tremelling M. (1972). The kinetics and mechanism of the decomposition of potassium cyanide in aqueous alkaline medium. Hydrolysis of the simplest nitrile. J Org Chem 37(6):914-916. 


\title{
Hydrogen Fluoride (HF) $\quad$ 7664-39-3
}

\section{ENVIRONMENTAL TRANSPORT, DISTRIBUTION, AND TRANSFORMATION}

\begin{abstract}
Air: Hydrogen fluoride is the most abundant gaseous fluoride released into the atmosphere. It reacts with many materials both in vapor and in aerosols. For example, hydrogen fluoride reacts with silica, forming silicon tetrafluoride. However, no information was found on the reactions of hydrogen fluoride with common atmospheric species or estimates of its overall atmospheric half-life. The predominant mode of degradation of inorganic fluorides in the air is hydrolysis. Silicon tetrafluoride, a major industrial pollutant, reacts with water vapor in air to form hydrated silica and fluorosilicic acid. Sulfur hexafluoride, a gaseous dielectric for electrical and electronic equipment, reacts with water at elevated temperatures $\left(>850^{\circ} \mathrm{C}\right)$ to form sulfuric acid and hydrogen fluoride (Guo et al. 2001). Molecular fluorine hydrolyzes to form hydrogen fluoride and oxygen. Hydrolysis of uranium hexafluoride, which is used in nuclear power applications, also produces hydrogen fluoride as well as nonvolatile uranyl fluoride. These compounds are then removed from the atmosphere by condensation or nucleation processes (NAS 1971). Fluorides emitted by industries in particulate matter are stable compounds that do not readily hydrolyze.
\end{abstract}

Water: Contrary to traditional thought, hydrogen fluoride, a very weak acid in dilute solution, is dissociated in solution, but forms tight ion pairs $\mathrm{F}-. . . \mathrm{H}^{+}-\mathrm{OH}_{2}$, unique to $\mathrm{F}^{-}$, which reduce the thermodynamic activity coefficient of $\mathrm{H}_{3} \mathrm{O}^{+}$(Cotton et al. 1999). In natural water, fluoride ions form strong complexes with aluminum, and fluorine chemistry in water is largely regulated by aluminum concentration and $\mathrm{pH}$. Below $\mathrm{pH} 5$, fluorine is almost entirely complexed with aluminum and consequently, the concentration of free $\mathrm{F}^{-}$is low. As the $\mathrm{pH}$ increases, $\mathrm{Al}-\mathrm{OH}$ complexes dominate over $\mathrm{Al}-\mathrm{F}$

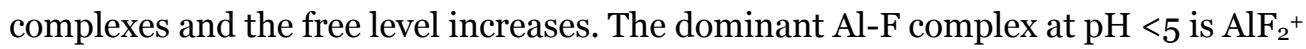
(Skjelkvale 1994). In the absence of aluminum, dissolved fluorides are usually present as free $\mathrm{F}^{-}$at neutral $\mathrm{pH}$ (Bell et al. 1970). As the $\mathrm{pH}$ decreases, the proportion of $\mathrm{F}^{-}$ decreases, while $\mathrm{HF}_{2}{ }^{-}$and undissociated hydrogen fluoride increase. Levels of undissociated hydrogen fluoride also increase in concentrated solutions. Fluorine can form stable complexes with calcium and magnesium, which are present in sea water. Using the stability constants valid for sea water, $51.0 \%$ of fluorine will be present as free $\mathrm{F}^{-}, 47.0 \%$ as $\mathrm{MgF}^{+}$, and $2.0 \%$ as $\mathrm{CaF}^{+}$(Stumm and Morgan 1981). Calcium carbonate precipitation dominates the removal of dissolved fluoride from sea water. Fluoride is incorporated into the calcium salt structure and is removed from solution when the latter precipitates. 
Sediment and Soil: Fluoride occurs in soil as a variety of minerals and complexes with aluminum, iron, and calcium. At low $\mathrm{pH}$, aluminum complexes, $\mathrm{AlF}_{3}, \mathrm{AlF}_{2}{ }^{+}$, and $\mathrm{AlF}_{2}{ }^{+}$, are the dominant dissolved species, and the availability of these soluble complexes increases with decreasing pH (Fung et al. 1999; Shacklette et al. 1974). This explains why more acidic soils have both higher water-soluble fluoride and higher extractable aluminum levels. In certain soils in which calcium is present mostly as calcium fluoride and in which there is sufficient alumina, fluoride is fixed by the formation of relatively insoluble aluminum fluorosilicate, $\mathrm{Al}_{2}\left(\mathrm{SiF}_{6}\right)_{3}$ (Brewer 1966).

Transport and Partitioning: In the atmosphere, gaseous hydrogen fluoride is absorbed by atmospheric water (rain, clouds, fog, snow) forming an aerosol or fog of aqueous hydrofluoric acid. It will be removed from the atmosphere primarily by wet deposition (including rainout or in-cloud scavenging and washout or below-cloud scavenging). Particulate fluorides are similarly removed from the atmosphere and deposited on land or surface water by wet and dry deposition. Atmospheric precipitation weathers crustal rocks and soil, but dissolves out very little fluoride; most of the fluoride mobilized during weathering is bound to solids such as clays. Upon reaching bodies of water, fluorides gravitate to the sediment (Carpenter 1969). Most of the fluorides in the oceans are received from rivers; a lesser amount comes from atmospheric deposition. Losses occur in aerosols to the atmosphere and incorporation into the tissue of aquatic organisms. Fluorides have been shown to accumulate in some marine aquatic organisms.

\section{Regulatory}

MCL: $4 \mathrm{mg} / \mathrm{L}$ (fluoride)

MCL (Drinking Water): None

Universal Treatment Standards (UTS): $0.29 \mathrm{mg} / \mathrm{l}^{2}$ in wastewater standards

Controlling Water Quality Criterion for Surface Water:

Basis: Aquatic Toxicity Criterion Concentration: 21 ug/L Criterion Quantitation Limit: 5 ug/L

EPA RfD: $0.0005 \mathrm{mg} / \mathrm{kg}$-day

EPA RfC: $0.000002 \mathrm{mg} / \mathrm{m}^{3}$

$\begin{array}{cccc}\text { PRG: residential soil } \mathrm{mg} / \mathrm{kg} & \text { industrial soil } \mathrm{mg} / \mathrm{kg} & \text { ambient air } \mathrm{ug} / \mathrm{m} & \text { tap water } \mathrm{ug} / \mathrm{L} \\ 0.1 & 0.345 & 0.21 & 0.042\end{array}$ 


\section{Remediation}

Reaction with excess lime followed by lagooning and either recovery or landfill disposal of the separated calcium fluoride. Alternatively, hydrogen can be recovered and recycled in many cases. In accordance with 40CFR165 recommendations for the disposal pesticides and pesticide containers. Must be disposed properly by following package label directions or by contacting your state pesticide or environmental control agency or by contacting your regional EPA office. Consult with environmental regulatory agencies for guidance on acceptable disposal practices. . Generators of waste containing this contaminant $(\geq 100$ $\mathrm{kg} / \mathrm{mo}$ ) must conform to EPA regulations governing storage, transportation, treatment, and waste disposal (Sittig 2002).

\section{References}

Bell ME, Largent EJ, Ludwig TG. (1970). The supply of fluorine to man. World Health Organization Monograph Series 59:17-74.

Brewer RF. (1966). Fluorine. In: Chapman HD, ed. Diagnostic criteria for plants and soils. Riverside, CA: Division of Agricultural Science, University of California, 180-195.

Carpenter R. (1969). Factors controlling the marine geochemistry of fluorine. Geochim Cosmochim Acta 33:1153-1167.

Cotton FA, Wilkinson G, Murillo CA. (1999). Advanced inorganic chemistry, 6th ed. New York: John Wiley \& Sons, Inc., 62-64.

Fung KF, Zhang ZQ, Wong JWC. (1999). Fluoride contents in tea and soil from tea plantations and the release of fluoride into tea liquor during infusion. Environ Pollut 104:197-205.

Guo Z, Moisley RB, Wasson SJ. (2001). Dissociation of sulfur hexafluoride tracer gas in the presence of an indoor combustion source. J Air Waste Manage Assoc 51:616-622.

National Acadamy of Science (NAS). (1971). Biologic effects of atmospheric pollutants: Fluorides. Washington, DC: National Academy of Sciences, National Research Council, Committee on Biologic Effects of Atmospheric Pollutants, 239.

Shacklette HT, Boerngen JG, Keith JR. (1974). Selenium, fluorine, and arsenic in surficial materials of the conterminous United States. Washington, DC: U.S. Department of the Interior. Geological Survey Circular 692. 
Skjelkvale BL. (1994). Factors influencing fluoride concentrations in Norwegian Lakes. Water Air Soil Pollut 77:151-167.

Sittig, M. 2002. Handbook of Toxic and Hazardous Chemicals and Carcinogens, 2002. 4th ed.Vol 1 A-H Norwich, NY: Noyes Publications. p. 1285

Stumm W, Morgan JJ. (1981). Aquatic chemistry. 2nd edition. New York: John Wiley and Sons, 365-367. 


\title{
Mercuric Chloride $\quad \mathbf{7 4 8 7 - 9 4 - 7}$
}

\section{ENVIRONMENTAL TRANSPORT, DISTRIBUTION, AND TRANSFORMATION}

\begin{abstract}
Air: Mercury is released to the environment by both natural processes (e.g., volcanic activity and weathering of mercury-containing rocks) and anthropogenic sources. Anthropogenic releases are primarily to the atmosphere. The primary form of atmospheric mercury, metallic mercury vapor $(\mathrm{Hg} 0)$, is oxidized by ozone to other forms (e.g., $\mathrm{Hg}^{+2}$ ) and is removed from the atmosphere by precipitation (Brosset 1991). The oxidation/reduction of mercury with dissolved ozone, hydrogen peroxide, hypochlorite entities, or organoperoxy compounds or radicals may also occur in the atmosphere (Schroeder et al. 1991). The overall residence time of elemental mercury in the atmosphere has been estimated to be 6 days to 2 years, although in clouds, a fast oxidation reaction on the order of hours may occur between elemental mercury and ozone. Some mercury compounds, such as mercuric sulfide, are quite stable in the atmosphere as a result of their binding to particles in the aerosol phase (Lindqvist 1991). Other mercury compounds, such as mercuric hydroxide $\left(\mathrm{Hg}[\mathrm{OH}]_{2}\right)$, which may be found in the aqueous phase of the atmosphere (e.g., rain), are rapidly reduced to monovalent mercury in sunlight (Munthe and McElroy 1992). The main atmospheric transformation process for organomercurials appears to be photolysis (EPA 1984; Johnson and Bramen 1974).
\end{abstract}

Water: The most important transformation process in the environmental fate of mercury in surface waters is biotransformation. Photolysis of organomercurials may also occur in surface waters, but the significance of this process in relation to biotransformation is not clear (Callahan et al. 1979). Any form of mercury entering surface waters can be microbially converted to methylmercuric ions, given favorable conditions. Sulfur-reducing bacteria are responsible for most of the mercury methylation in the environment (Gilmour and Henry 1991), with anaerobic conditions favoring their activity (Regnell and Tunlid 1991). Yeasts, such as Candida albicans and Saccharomyces cerevisiae, whose growth is favored by low $\mathrm{pH}$ conditions, are able to methylate mercury and are also able to reduce ionic mercury to elemental mercury (Yannai et al. 1991). Methyl cobalamine compounds produced by bacterial synthesis appear to be involved in the nonenzymatic methylation of inorganic mercury ions (Regnell and Tunlid 1991). The rate of methylmercury formation by this process is largely determined by the concentration of methyl cobalamine compounds, inorganic mercuric ions, and the oxygen concentration of the water, with the rate increasing as the conditions become anaerobic. Volatile elemental mercury may be formed through the demethylation of methylmercury or the reduction of inorganic mercury, with anaerobic conditions again favoring the demethylation of the methylmercury (Barkay et al. 1989; Callahan et al. 1979; Regnell and Tunlid 1991). Increased dissolved organic carbon levels reduce methylation of mercury in 
the water column (Gilmour and Henry 1991), possibly as a result of the binding of free mercury ions to the dissolved organic carbon at low $\mathrm{pH}$, thus reducing their availability for methylation, or the dissolved organic carbon may inhibit the methylating bacteria (Miskimmin et al. 1992). Alternatively, low pH favors the methylation of mercury in the water column, particularly in acid deposition lakes, while inhibiting its demethylation (Gilmour and Henry 1991). It has also been shown that the methylation rate is not affected by addition of sulfate in softwater lakes (Kerry et al. 1991).

Sediment and Soil: Mercury compounds in soils may undergo the same chemical and biological transformations described for surface waters. Mercuric mercury usually forms various complexes with chloride and hydroxide ions in soils; the specific complexes formed depend on the $\mathrm{pH}$, salt content, and composition of the soil solution. Formation and degradation of organic mercurials in soils appear to be mediated by the same types of microbial processes occurring in surface waters and may also occur through abiotic processes (Andersson 1979). Elevated levels of chloride ions reduce methylation of mercury in river sediments, sludge, and soil (Olson et al. 1991), although increased levels of organic carbon and sulfate ions increase methylation in sediments (Gilmour and Henry 1991). In freshwater and estuarine ecosystems, the presence of chloride ions (0.02 M) may accelerate the release of mercury from sediments (Wang et al. 1991).

Biota: Bioconcentration factors of 10,000 and 40,000 have been obtained for mercuric chloride and methylmercury with oyster (EPA 1986).

\section{Environmental partitioning}

The natural global bio-geochemical cycling of mercury is characterized by degassing of the element from soils and surface waters, followed by atmospheric transport, deposition of mercury back to land and surface waters, and sorption of the compound to soil or sediment particulates. Mercury deposited on land and open water is in part revolatilized back into the atmosphere. This emission, deposition, and revolatilization create difficulties in tracing the movement of mercury to its sources (WHO 1990). Particulate-bound mercury can be converted to insoluble mercury sulfide and precipitated or bioconverted into more volatile or soluble forms that re-enter the atmosphere or are bioaccumulated in aquatic and terrestrial food chains (EPA 1984b).

Mercury is transformed in the environment by biotic and abiotic oxidation and reduction, bioconversion of inorganic and organic forms, and photolysis of organomercurials. Inorganic mercury can be methylated by microorganisms indigenous to soils, fresh water, and salt water. This process is mediated by various microbial populations under both aerobic and anaerobic conditions. The most probable mechanism for this reaction involves the nonenzymatic methylation of mercuric mercury ions by methylcobalamine compounds produced as a result of bacterial synthesis. Mercury forms stable complexes with organic compounds. Monoalkyl mercury 
compounds (e.g., methylmercuric chloride) are relatively soluble; however, the solubility of methylmercury is decreased with increasing dissolved organic carbon content, indicating that it is bound by organic matter in water (Miskimmin 1991). Dialkyl mercury compounds (e.g., dimethylmercury) are relatively insoluble (Callahan et al. 1979; EPA 1984). Dimethylmercury is volatile, although it makes up less than $3 \%$ of the dissolved gaseous mercury found in water (Andersson et al. 1990; Vandal et al. 1991).

\section{Regulatory}

MCL (Drinking Water): $0.002 \mathrm{mg} / \mathrm{L} .(\mathrm{Hg})$

Universal Treatment Standards (UTS) for Hg others: $0.15 \mathrm{mg} / \mathrm{l}^{2}$ in wastewater standards 0.025mg/L TCLP in nonwastewater standards

EPA RfD: 0.0003 mg/k/day (U.S. EPA, 1995)

EPA RfC: $\quad \mathrm{mg} / \mathrm{m}^{3}$

PRG: residential soil $\mathrm{mg} / \mathrm{kg}$ industrial soil $\mathrm{mg} / \mathrm{kg}$ ambient air $\mathrm{ug} / \mathrm{m} \quad$ tap water $\mathrm{ug} / \mathrm{L}$ $\begin{array}{llll}6.1 & 88 & 0.31 & 3.6\end{array}$

Soil PQL (mercury): 0.1mg/kg

\section{Remediation}

Mercury removal from waste water can be accomplished by these processes: BMS process; Chlorine is added to the waste water, oxidizing any mercury present to the ionic state. The BMS adsorbent (an activated carbon concentrate of sulfur compound on its surface) is used to collect ionic mercury. The spent adsorbent is then distilled to recover the mercury, leaving a carbon residue for reuse or disposal. TMR IMAC Process; Waste water is fed into a reactor, whereby a slight excess of chlorine is maintained, oxidizing any mercury present to ionic mercury. The liquid is then passed through the TMR IMAC ionexchange resin where mercury ions are adsorbed. The mercury is then stripped from the spent resin with hydrochloric acid solution (Environmental Canada).

\section{References}

Andersson A. (1979). Mercury in soils. In: Nriagu JO, ed. The biogeochemistry of mercury in the environment. New York, NY: Elsevier/North Holland Biomedical Press, 79-112. 
Barkay T, Liebert C, Gillman M. (1989). Environmental significance of the potential for mer(TN21)-mediated reduction of $\mathrm{Hg}^{2+}$ to $\mathrm{HgO}$ in natural waters. Appl Environ Microbiol 55(5):1196-1202.

Brosset C, Lord E. (1991). Mercury in precipitation and ambient air: A new scenario. Water Air Soil Pollut 56:493-506.

Callahan MA, Slimak MW, Gabel NW. (1979). Water related environmental fate of 129 priority pollutants, introduction and technical background, metals and inorganics, pesticides and PCBs. Washington, D.C: U.S. Environmental Protection Agency, Office of Water Waste and Management. Document no. EPA 440/4-79-029a., 14-1 -14-15.

Environment Canada; Tech Info for Problem Spills: Mercury (Draft) p.59 (1982).

EPA. (1984). Mercury health effects updates: Health issue assessment. Final report. Washington, DC: U.S. Environmental Protection Agency, Office of Health and Environmental Assessment. Document no. EPA 600/8-84-019F.

Gilmour CC, Henry EA. (1991). Mercury methylation in aquatic systems affected by acid deposition. Environmental Pollution 71(2-4):131-169.

Johnson DC, Bramen RS. (1974). Distribution of atmospheric mercury species near the ground. Environ Sci Technol 8:1003-1009.

Kerry A, Welbourn PM, Prucha B. (1991). Mercury methylation by sulphate-reducing bacteria from sediments of an acid stressed lake. Water Air Soil Pollut 56:565-575.

Lindqvist O. (1991). Mercury in the Swedish environment: 4. Emissions of mercury to the atmosphere. Water, Air, Soil Pollution 55(1-2):23-32.

Miskimmin BM. (1991). Effects of natural levels of dissolved organic carbon (DOC) on methyl mercury formation and sediment water partitioning. Bull Environ Contam Toxicol 47(5):743-750.

Miskimmin BM, Rudd JWM, Kelly CA. (1992). Influence of dissolved organic carbon, pH, and microbial respiration rates on mercury methylation and demethylation in lake water. Can J Fish Aquat Sci 49(1):17-22.

Munthe J, McElroy WJ. (1992). Some aqueous reactions of potential importance in the atmospheric chemistry of mercury. Atxos Environ Part A Gen Top 26(4):553-557. 
Olson BH, Cayless SM, Ford S. (1991). Toxic element contamination and the occurrence of mercury-resistant bacteria in mercury contaminated soil, sediments, and sludges. Arch Environ Contam Toxicol 20(2):226-233.

Regnell O, Tunlid A. (1991). Laboratory study of chemical speciation of mercury in lake sediment and water under aerobic and anaerobic conditions. Appl Environ Microbiol 57(3):789-795.

Schroeder WH, Yarwood G, Niki H. (1991). Transformation processes involving mercury species in the atmosphere - results from a literature survey. Water, Air, Soil Pollution 56:653-666.

USEPA/OWRS (1986) Quality Criteria for Water 1986 EPA 440/5-86-001.

Wang JS, Huang PM, Liaw WK. (1991) Kinetics of the desorption of mercury from selected fresh water sediments as influenced by chloride. Water, Air, Soil Pollution 56:533-542.

WHO (1990) Methyl mercury Vol. 101. Geneva, Switzerland: World Health Organization, International Programme on Chemical Safety.

Yannai S, Berdicevsky I, Duek L (1991) Transformations of inorganic mercury by candidaalbicans and saccharomyces-cerevisiae. Appl Environ Microbiology 57(1):245-247. 


\section{Methamidophos (O, S-Dimethyl phosphoramidothioate) 10265-92-6}

\section{ENVIRONMENTAL TRANSPORT, DISTRIBUTION, AND TRANSFORMATION}

Air: Based on a reported vapor pressure for methamidophos of $3 \mathrm{X}^{-4} \mathrm{O}^{-4} \mathrm{~mm} \mathrm{Hg}$ at $30 \mathrm{deg}$ C (Bidleman 1988), methamidophos is expected to exist almost entirely in the vapor phase in the ambient atmosphere (Tomlin 1997). The rate constant for the reaction of methamidophos with photochemically produced hydroxyl radicals is estimated to be $2.7 \mathrm{X} \mathrm{O}^{-11} \mathrm{cu} \mathrm{cm} / \mathrm{molecule}-\mathrm{sec}$ at $25 \mathrm{deg} \mathrm{C}$, which corresponds to a half-life of 4.6 hours for an atmospheric hydroxyl radical concentration of $5 \mathrm{X} 10+5$ molecules/cu $\mathrm{cm}$ (Meylan and Howard 1993).

Water: A Koc value of 5 indicates that methamidophos is not expected to adsorb to suspended solids and sediment (Swann et al. 1983). Volatilization from water surfaces is not expected (Lyman et al. 1990) based upon an estimated Henry's Law constant of 8.7X10-10 atm-cu m/mole(SRC), developed using a fragment constant estimation method (Meylan and Howard 1991). According to a classification scheme (Franke et al 1994), an estimated BCF of 3.1, from its log Kow of -0.8 (Tomlin 1997) and a regression-derived equation (Meylan et al.1999), suggests the potential for bioconcentration in aquatic organisms is low. Half-lives for the hydrolysis of methamidiophos at $22 \operatorname{deg} \mathrm{C}$ and $\mathrm{pH} 4$, 7 , and 9 have been reported to be 1.8 years, 120 hours, and 70 hours, respectively (Tomlin 1997). The photolysis rate in water is 0.0079 per day (USDA 2003), corresponding to a half-life of 87 days.

Soil and Sediment: A Koc value of 5 (Wauchope et al. 1991), indicates that methamidophos is expected to have very high mobility in soil (Swann et al. 1983). Volatilization of methamidophos from moist soil surfaces is not expected to be an important fate process(SRC) given an estimated Henry's Law constant of 8.7X10 ${ }^{-10}$ atm$\mathrm{cu} \mathrm{m} / \mathrm{mole}$ (SRC), using a fragment constant estimation method (Tomlin 1997).

Methamidophos is not expected to volatilize from dry soil surfaces (SRC) based upon a vapor pressure of 3.5 X10-5 mm Hg (Tomlin 1997). Average half-lives in soil under aerobic and anaerobic conditions are 4 and 7.5 days, respectively (USDA 2003). The photolysis rate in soil is reported as 0.210 per day (USDA 2003), corresponding to a half-life of 3.3 days (SRC).

Biota: An estimated BCF of 3.1 was calculated for methamidophos, using a log Kow of o.8 (Tomlin 1997) and a regression-derived equation (Meylan et al.1999). This BCF 
suggests the potential for bioconcentration in aquatic organisms is low (Franke et al. 1993).

Environmental partitioning: Methamidophos' production may result in its release to the environment through various waste streams; its use as an insecticide and acaricide will result in its direct release to the environment. If released to air, a vapor pressure of $3.5 \mathrm{X}^{-5} \mathrm{~mm} \mathrm{Hg}^{-5} 25 \mathrm{deg} \mathrm{C}$ indicates methamidophos will exist in both the vapor and particulate phases in the ambient atmosphere. Vapor-phase methamidophos will be degraded in the atmosphere by reaction with photochemically-produced hydroxyl radicals; the half-life for this reaction in air is estimated to be 12 hours. Particulate-phase methamidophos will be removed from the atmosphere by wet and dry deposition. If released to soil, methamidophos is expected to have very high mobility based upon a Koc of 5. Volatilization from moist soil surfaces is not expected to be an important fate process based upon an estimated Henry's Law constant of 8.7 ${\mathrm{X} 10^{-10}}^{\mathrm{atm}-\mathrm{cu}} \mathrm{m} / \mathrm{mole}$. Volatilization The log Pow of -0.8 and the animal metabolism and feeding studies suggest that methamidophos should not be described as fat-soluble (Methamidophos 100).

\section{Regulatory}

MCL (Drinking Water): None

Drinking Water Remedial Target: 1.8 ug/L

Universal Treatment Standards (UTS): none

EPA Rf D: $0.00005 \mathrm{mg} / \mathrm{kg}$-day

EPA RfC: none

PRG: residential soil $\mathrm{mg} / \mathrm{kg}$ industrial soil $\mathrm{mg} / \mathrm{kg}$ ambient air $\mathrm{ug} / \mathrm{m} \quad$ tap water $\mathrm{ug} / \mathrm{L}$ $\begin{array}{llll}3.1 & 44 & 0.18 & 1.8\end{array}$

\section{Remediation}

Spills and powders should be cleaned up using a dustless method (e.g., by a vacuum cleaner suitable for use with toxic dusts). Alternatively, mix with damp saw-dust and place in a separate container for subsequent disposal. Dry brushing should not be carried out, as this creates dust clouds (ICPS). 
Large amounts should be incinerated at high temperature in a unit with effluent gas scrubbing. When no incinerator is available, bury in an approved dump, or in an area where there is no risk of contamination

of surface or groundwater. Before burying, liberally mix with sodium carbonate (washing soda) crystals, to help neutralize the product, and with soil rich in organic matter (ICPS).

\section{Reference}

Atkinson, R., J. Phys. Chem. Ref. Data, 1989, Monograph 1, 1.

Bidleman, T.F. (1988). “Atmospheric processes: Great Lakes Pesticide

Report"Environmental Science and Technology. 22(4):361-367.

Carazo, E., Constenia, MA, Fuentes, G., Moza, PN. (1984) Studies of methamidophos-C14 in Costa Rican vegetables and soils. Chemosphere 13: 939-46.

Eisenreich SJ, Looney BB, Thornton JD. (1981) Airborne organic contaminants in the Great Lakes ecosystem. Environ Sci Technol 15:30-8.

Franke, C., G. Studinger, G. Berger, S. Böhling, U. Bruckmann, D. Cohors-Fressenborg and U. Jöhncke, (1994) The assessment of bioaccumulation. Chemosphere 29: 1501-14.

IPCS, International Programme On Chemical Safety; Health and Safety Guide No. 79.

Lyman, WJ, WF Reehl, and DH Rosenblatt; (1990). Handbook of Chemical Property Estimation Methods. Washington, DC: Amer Chem Soc pp. 4-9, 15-1 to 15-29.

Methamidophos (100).

www.fao.org/ag/AGP/AGPP/Pesticid/JMPR/Download/2003_eva/

methamidophos\%202003.pdf - (Assessed May 26, 2009)

Meylan, W.M. and Howard, P.H. (1991). Bond contribution method for estimating Henry's Law Constants. Environ. Toxicol. Chem. 10: 1283-93.

Meylan, W., Howard, P.H. and Boethling, R.S. (1992). Molecular topology/fragment contribution method for predicting soil sorption coefficients. Environ. Sci. Technol. 26: 1560-6. 
Meylan, W. M. and P. H. Howard (1993). Reliability: Estimate based on known qualitative structure-activity relationships Chemosphere, 26:2293-99.

Meylan, WM, Howard, PH, Boethling, RS, Aronson, D, Printup, H, Gouchie, S. (1999) Improved method for estimating bioconcentration/bioaccumulation factor from octanol/water partition coefficient. Environ Toxicol Chem 18(4):664-72.

Swann R, Laskowski D, and McCAll P, (1983). A rapid method for the estimation of the environmental parameters octanol/water partition coefficient, soil sorption constant, water to air ratio, and water solubility. Residue Rev 85:18-28.

Tomlin CDS, ed; (1997). The Pesticide Manual. 11th ed. Farnham, Surrey, UK: British Crop Protection Council p. 808.

USDA; ARS Pesticide Properties Database on Methamidophos (10265-92-6). Available from the Database Query page at http://www.arsusda.gov/acsl/services/ppdb/ppdb3.html as of July 2, 2003.

Worthing CR and Walker SB, eds; (1987) . The Pesticide Manual 8th ed.; Suffolk, England: Lavenham Press Ltd . 


\section{Methyl Bromide (Bromomethane) 74-83-9}

\section{ENVIRONMENTAL TRANSPORT, DISTRIBUTION, AND TRANSFORMATION}

Air: According to a model of gas/particle partitioning of semivolatile organic compounds in the atmosphere (Bidleman 1988), methyl bromide, which has a vapor pressure of 1,620 mm Hg at 25 deg C (Daubert and Danner 1989), will exist in the gas phase (SRC). Gas-phase methyl bromide is degraded in the atmosphere by reaction with photochemically-produced hydroxyl radicals; the half-life for this reaction in air is about one year, calculated from its rate constant of $4.0 \mathrm{X}_{10}^{-14} \mathrm{cu} \mathrm{cm} / \mathrm{molecule-sec}$ at $25 \mathrm{deg} \mathrm{C}$ (Atkinson 1989). Methyl bromide is not expected to undergo direct photolysis due to the lack of absorption in the environmental UV spectrum (>290 nm) (Robbins 1976). Upward diffusion of methyl bromide to the stratosphere is believed to be the dominant loss mechanism of this compound from the troposphere (Robbins 1976). In the stratosphere, into which lower wavelength light can penetrate, photolysis is expected to be the predominant removal mechanism of methyl bromide (Robbins 1976).

Water: Koc values ranging from 9 to 22 (USDA 2001), indicate that methyl bromide is not expected to adsorb to suspended solids and sediment (Swann et al 1983),. Volatilization from water surfaces is expected based upon a Henry's Law constant of 7.34X10-3 atm-cu m/mole (Yates and Gan 1998). Using this Henry's Law constant and an estimation method (Lyman et al 1990), volatilization half-lives for a model river and model lake are 1.o hrs and 3.9 days, respectively. An estimated BCF of 2 (SRC), from its log Kow (Hansch et al 1995) and a regression-derived equation (Meylan et al 1999), suggests the potential for bioconcentration in aquatic organisms is low (Franke et al 1995). Chemical hydrolysis is the primary degradation mechanism for methyl bromide in water. The hydrolysis rate constant of methyl bromide at $25 \mathrm{deg} \mathrm{C}$ and $\mathrm{pH}$ 7 is $4.1{\mathrm{X} 10^{-7}}^{-1} \mathrm{sec}$ which translates into a half-life of 20 days (Mabey and Mill 1978). Another reported hydrolysis rate constant for methyl bromide is $3 \mathrm{X}_{10} \mathrm{O}^{-7} 1 / \mathrm{sec}$ at $25 \mathrm{deg}$ $\mathrm{C}$ (Castro and Belser 1981), which translates into a half-life of 26.7 days. The products of methyl bromide hydrolysis are methanol and bromide ion (Castro and Belser 1981).

Soil/Sediment: Koc values ranging from 9 to 22 (USDA 2001) indicate that methyl bromide is expected to have very high mobility in soil (Swann et al 1983),. Volatilization of methyl bromide from moist soil surfaces is an important fate process given a Henry's Law constant of 7.34X10 ${ }^{-3}$ atm-cu $\mathrm{m} / \mathrm{mole}$ (Yates and Gan 1996). The volatilization of methyl bromide from dry soil surfaces is an important removal mechanism from soil (SRC) based upon a vapor pressure of 1,620 mm Hg at $25 \mathrm{deg} C$ (Daubert and Danner 1989). In soil, chemical reactions, likely nucleophilic 
substitutions on soil organic matter, were identified as the predominate pathway through which methyl bromide was degraded (Gan and Yates 1996). For example, methyl bromide reacts rapidly with aniline (a model used to simulate reactions with organic matter in soil) compared to direct hydrolysis with water (Gan and Yates 1996); the degradation half-lives of methyl bromide with water and aniline are 20 days and 2.9 days at 24 deg C, respectively (Gan and Yates 1996). The oxidation of 14C-methyl bromide to 14CO2 was measured in field experiments with soils collected from two strawberry plots fumigated with mixtures of methyl bromide and chloropicrin (Miller et al 1997). Degradation of methyl bromide by chemical and/or biological processes accounted for 20 to $50 \%$ of the loss of methyl bromide during fumigation (Miller et al 1997 ) with the remainder of methyl bromide loss due to volatilization.

Environmental Partitioning: Transformation or degradation of $\mathrm{MeBr}$ is an irreversible process that depletes $\mathrm{MeBr}$ from the soil-water-air system before it reaches the soil surface and volatilizes into the air. Extremely rapid transformation may deplete $\mathrm{MeBr}$ concentrations so quickly that efficacy is compromised. The actual transformation of $\mathrm{MeBr}$ in an agricultural soil is the sum of its hydrolysis in water, reactions with soil constituents, and decomposition by soil microorganisms.

\section{Regulations}

MCL (Drinking Water): None

GPS - Groundwater protection standard (DWEL): $0.1 \mathrm{mg} / \mathrm{L}$

Universal Treatment Standards (UTS): $0.1 \mathrm{mg} / \mathrm{l} 2$ in wastewater standards and $15 \mathrm{mg} / \mathrm{kg} 3$

Groundwater Water Quality Criterion : $10 \mathrm{ug} / \mathrm{L}$

PQL: 2

EPA Rf D: $0.0014 \mathrm{mg} / \mathrm{kg}$-day

EPA RfC: $0.005 \mathrm{mg} / \mathrm{m}^{3}$

PRG: residential soil mg/kg industrial soil mg/kg ambient air ug/m tap water ug/L 3.9 


\section{Remediation}

Generators of waste (equal to or greater than $100 \mathrm{~kg} / \mathrm{mo}$ ) containing this contaminant, EPA hazardous waste number U029, must conform with USEPA regulations in storage, transportation, treatment and disposal of waste (40 CFR).

\section{References}

Altshuller AP; (1980).Comments on the Lifetimes of Organic Molecules in Air. USEPA-600/9-80003.

Atkinson R; (1989) Kinetics and mechanisms of the gas-phase reactions of the hydroxyl radical with organic compounds. J Phys Chem Ref Data Monograph 1.

Bidleman T. F. (1988) Atmospheric processes. Envir. Sei. Technol. 22:361-367.

Castro CE, Belser NO (1981) Photohydrolysis of methyl bromide and chloropicrin. J Agric Food Chem 29:1005-1008. 40 CFR 240-280, 300-306, 702-799 (7/1/2000)

Daubert TE, Danner RP; (1989) Physical and Thermodynamic Properties of Pure Chemicals: Data Compilation. Design Inst Phys Prop Data, Amer Inst Chem Eng. NY, NY: Hemisphere Pub Corp.

Franke, C., G. Studinger, G. Berger, S. Böhling, U. Bruckmann, D. Cohors-Fressenborg and U. Jöhncke (1994) The assessment of bioaccumulation. Chemosphere 29: 1501-14

Gan JY, Yates SR (1996) Degradation and phase-partition of methyl iodide in soil. J Agric Food Chem 44:4001-4008.

Hansch, C., Leo, A., and Hoekman, D. (1995) Exploring QSAR. Hydrophobic, Electronic, and Steric Constants. ACS Prof Ref Book. Heller SR, consult. ed.,Washington, DC: American Chemical Society, p. 3.

Lyman, WJ, WF Reehl, and DH Rosenblatt (1990) Handbook of Chemical Property Estimation Methods. Washington, DC: American Chemical Society pp. 4-9.

Mabey W, Mill T (1978) Critical review of hydrolysis of organic compounds in water under environmental conditions. J Phys Chem Ref Data 7:383-415. 
Meylan, WM; Howard, PH; and Boethling, RS (1999) Improved method for estimating bioconcentration/bioaccumulation factor from octanol/water partition coefficient. Environ. Toxicol. Chem. 18: 664-672.

Miller LG, Connell TL, Guidetti JR, Oremland RS (1997) Bacterial oxidation of methyl bromide in fumigated agricultural soils. Appl Environ Microbiol 63:4346-4354.

Robbins DE; (1976)Photodissociation of Methyl Chloride and Methyl Bromide in the Atmosphere. Geophys Res Lett 3: 213-6.

Syracuse Research Corporation (SCR). (1996). ChemFate Database. Syracuse, NY.

Swann, R.L., Laskowski, D.A., McCall, P.J., van der Kuy, K. and Dishburger, H.J. (1983) A rapid method for estimation of the environmental parameters octanol/water partition coefficient, soil sorption constant water to air ratio and water solubility. Residue Rev. 85, pp. 17-28. (http://toxnet.nlm.nih.gov/cgi-bin/sis/search/r?dbs+hsdb:@term+@rn+6o34-4).

USDA; ARS Pesticide Properties Database on Methyl Bromide (74-83-9). Available from the Database Query page at http://www.arsusda.gov/acsl/services/ppdb/ppdb3.html as of July 17, 2001.

Yates SR, Gan JY (1998) Volatility, adsorption, and degradation of propargyl bromide as a soil fumigant. J Agric Food Chem 46:755-761. 


\title{
Methyl Hydrazine $\quad 60-34-4$
}

\section{ENVIRONMENTAL TRANSPORT, DISTRIBUTION, AND TRANSFORMATION}

\begin{abstract}
Air: According to a model of gas/particle partitioning of semivolatile organic compounds in the atmosphere (Bidleman 1988), methylhydrazine, which has a measured vapor pressure of $50 \mathrm{~mm} \mathrm{Hg}$ at $25 \mathrm{deg} \mathrm{C}$ (Boublik et al. 1984), will exist solely as a vapor in the ambient atmosphere. Vapor-phase methylhydrazine is degraded in the atmosphere by reaction with photochemically-produced hydroxyl radicals; the half-life for this reaction in air is estimated to be about 6 hours calculated from its rate constant of $6.50 \times 10^{-11} \mathrm{cu}$ $\mathrm{cm} /$ molecule-sec at $25 \mathrm{deg} \mathrm{C}$ (Atkinson 1989). Assuming an ozone concentration of 7X10+11 molecules/cu cm, a maximum half-life of about $3.3 \mathrm{~min}$ for the reaction of methylhydrazine with ozone was estimated from a minimum rate constant of $5 \mathrm{X}^{-15} \mathrm{cu}$ $\mathrm{cm} /$ molecule sec (Tuazon et al. 1982). The half-life for the reaction between ozone and methylhydrazine was estimated to be $<1$ min during ozone pollution episodes and $<12$ min in the 'natural' troposphere (Tuazon et al. 1981). Reaction of methylhydrazine with ozone is expected to be the predominant fate of methylhydrazine in the atmosphere.
\end{abstract}

Water: An estimated Koc value of 18 (SRC), determined from a structure estimation method, indicates that methylhydrazine is not expected to adsorb to suspended solids and sediment (Swann et al. 1983), a. Methylhydrazine is a weak base with a pKa of 7.87 (Perrin 1965). This pKa value indicates that this compound will partially exist as a cation in water, and cations may adsorb to suspended solids and sediment more than neutral compounds (SRC). Volatilization of the protonated species will not occur since cations do not volatilize. Volatilization of the free base may occur (Lyman et al. 1990), based on an estimated Henry's Law constant of $3 \mathrm{X}_{10^{-6}} \mathrm{~atm}-\mathrm{cu} \mathrm{m} / \mathrm{mole}$ (SRC), calculated from its vapor pressure, $50 \mathrm{~mm} \mathrm{Hg}$ (Boublik et al.1984), and water solubility, $1 \mathrm{X}^{\circ} \mathrm{O}^{6} \mathrm{mg} / \mathrm{L}$ (O'Neil 2001).

Sediment: An estimated Koc value of 18, determined from a structure estimation method (Meylan et al. 1992, Hansch et al. 1995), indicates that methylhydrazine is not expected to adsorb to suspended solids and sediment (Swann et al. 1983).

Methylhydrazine is a weak base with a pKa of 7.87 (Perrin 1965). This pKa value indicates that this compound will partially exist as a cation in water, and cations may adsorb to suspended solids and sediment more than neutral compounds. Volatilization of the protonated species will not occur since cations do not volatilize. Volatilization of the free base may occur (Lyman et al. 1990), based on an estimated Henry's Law constant of

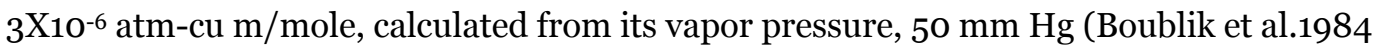
), and water solubility, $1 \mathrm{X}_{10} \mathrm{O}^{6} \mathrm{mg} / \mathrm{L}$. Based on this Henry's Law constant, the volatilization 
half-life from a model river ( $1 \mathrm{~m}$ deep, flowing $1 \mathrm{~m} / \mathrm{sec}$, wind velocity of $3 \mathrm{~m} / \mathrm{sec}$ ) (Perrin 1965 ) is estimated as 5 days. The volatilization half-life from a model lake (1 m deep, flowing $0.05 \mathrm{~m} / \mathrm{sec}$, wind velocity of $0.5 \mathrm{~m} / \mathrm{sec}$ ) (Lyman et al. 1990) is estimated as 62 days. According to a classification scheme (Franke et al. 1994), an estimated BCF of 3, from a log Kow of -1.05 (Hansch et al. 1995) and a regression-derived equation (Meylan et al. 1999), suggests the potential for bioconcentration in aquatic organisms is low. The estimated half-lives of methylhydrazine present at $9.5 \mathrm{mM}$ in pond and sea water are 18.0 and 24.1 days, respectively, and at $19.0 \mathrm{mM}$ are 13.1 days in both pond and sea water (Braun and Zirrolli 1983).

Soil: Based on a classification scheme (Swann et al. 1983), an estimated Koc value of 18 , determined from a structure estimation method (Meylan et al. 1992), indicates that methylhydrazine is expected to have very high mobility in soil. Methylhydrazine is a weak base with a pKa of 7.87 (Perrin 1965). This pKa value indicates that this compound will partially exist as a cation in moist soils, and cations may adsorb to soil more than neutral compounds. Volatilization of the protonated species from moist soils will not occur since cations do not volatilize. Volatilization of the free base may occur, based on an estimated Henry's Law constant of $3 \mathrm{X}_{10} \mathrm{O}^{-6} \mathrm{~atm}-\mathrm{cu} \mathrm{m} / \mathrm{mole}$, calculated from its vapor pressure, 50 mm Hg (Boublik et al. 1984), and water solubility, $1 \mathrm{X} \mathrm{O}^{6} \mathrm{mg} / \mathrm{L}$ (O'Neil 2001). Volatilization from dry soils may occur based on the vapor pressure of this compound (Boublik et al.1984). Approximately 0\%, 5\%, 20\%, and 8\% degradation of methylhydrazine was observed in a 1 hour soil column study using a cleaned sand (100\% sand), sandy soil (99.1\% sand, o.4\% clay, pH 6.1), organic soil (96.1\% sand, $1 \%$ clay, $1 \%$ carbon, pH 6.4) and clay (69.3\% sand, 27.95\% clay, pH 3.7) (Braun and Zirrolli 1983).

Biota: An estimated BCF value of 3 was calculated for methylhydrazine, using a measured log Kow of -1.05 (Hansch et al. 1995) and a regression-derived equation (Meylan et al. 1995). According to a classification scheme (Franke et al. 1994), this BCF value suggests that bioconcentration in aquatic organisms is low.

Environmental partitioning: Methylhydrazine's production and use as a rocket fuel, solvent and chemical intermediate may result in its release to the environment through various waste streams. If released to the atmosphere, methylhydrazine will exist solely in the vapor phase in the ambient atmosphere, based on a measured vapor pressure of 50 $\mathrm{mm} \mathrm{Hg}$ at $25 \mathrm{deg}$ C. Vapor-phase methylhydrazine is degraded in the atmosphere by reaction with photochemically-produced hydroxyl radicals and ozone with estimated halflives of about 6 hours and 1-12 minutes, respectively. If released to soil, an estimated Koc value of 18 suggests that methylhydrazine will have very high mobility in soil.

Methylhydrazine is a weak base having a pKa of 7.87, suggesting that it will partially exist in the protonated form in water and moist soils and the protonated form may adsorb to 
soils more than the free base. Volatilization from moist soil surfaces will not occur for the protonated species since cations do not volatilize. Volatilization may occur for the neutral species based on an estimated Henry's Law constant of $3 \mathrm{X}_{10^{-6}} \mathrm{~atm}-\mathrm{cu} \mathrm{m} / \mathrm{mole}$ (HSDB).

\section{Regulatory}

MCL (Drinking Water): (FL) FLORIDA $10 \mathrm{ug} / \mathrm{l}$

[USEPA/Office of Water; Federal-State Toxicology and Risk Analysis Committee (FSTRAC). Summary of State and Federal Drinking Water Standards and Guidelines $(11 / 93)$

Universal Treatment Standards (UTS): None

EPA RfD: none mg/kg-day

EPA RfC: none $\mathrm{mg} / \mathrm{m}^{3}$

Drinking Water Remedial Target: $0.22 \mu \mathrm{g} / \mathrm{L}$

PRG: residential soil mg/kg industrial soil $\mathrm{mg} / \mathrm{kg}$ ambient air ug/m3 tap water ug/L $\begin{array}{llll}0.44 & 2.2 & 0.0061 & 0.061\end{array}$

\section{Remediation}

The toxicity of methylhydrazine to microbial species was found to be sufficiently high to prevent its degradation by biological waste treatment (Kane and Williamson 1983). Large amounts of methylhydrazine, such as might be released from a spill, are not expected to biodegrade. However, biodegradation of lower methylhydrazine concern may occur. Methylhydrazine at $500 \mathrm{mg} / \mathrm{l}$, present in a wastewater mixture of hydrazine compounds, was incubated with an inoculum prepared from a trickling filter plant; following a 24 hour lag period, this mixture of compounds was biodegraded as measured by oxygen uptake (Wachinski and Farmwald 1980). Contaminated wastewater from the NASA Kennedy Space station was studied in batch cultures and trickle bed reactors to monitor the degradation rates of hydrazine containing rocket fuels (Nwankwoala et al. 1999a, b). The half-life of methylhydrazine in batch cultures (Rhodococcus B30 or Achromobacter sp.) and trickle bed reactors was about 2.5 and 12 days, respectively (Nwankwoala et al. 1999a). Biodegradation may be a significant removal process at low concentrations in soils or ambient waters, but at higher concentrations hydrazines are toxic to microorganisms (Nwankwoala et al. 1999b). Concentrations of hydrazine and 1,1dimethylhydrazine that reduced bacterial metabolism by 50\% ranged from 14.6 to 145 $\mathrm{mg} / \mathrm{L}$ and from 19.2 to 9,060 mg/L, respectively(ATSDR 1998). 


\section{References}

ATSDR; Agency for Toxic Substances and Disease Registry Toxicological Profile for Hydrazines. U.S. Department of Health \& Human Services, Atlanta, GA (1998).

Atkinson R; Journal Physic Chemical Reference Data. Monograph Vol 1. (1989). Journal of Physical and Chemical Reference Data Monographs \& Supplements (http://www.aip.org/pubs/books/jpcrd_books.html).

Bidleman, T.F. (1988). Atmospheric processes: Great Lakes Pesticide Report. Environmental Science and Technology. 22(4):361-367.

Boublik, T., Fried, V., \& Hala, E. (1984). The vapour pressures of pure substances. 2nd edition. Amsterdam, Netherlands: Elsevier Science Publication Vol. 17.

Braun BA and Zirrolli JA. (1983). Environ Fate of Hydrazine Fuels in Aqueous and Soil Environ Air Force Report No. ESL-TR-82-45, NTIS AD-A125813.

Franke, C., G. Studinger, G. Berger, S. Böhling, U. Bruckmann, D. Cohors-Fressenborg and U. Jöhncke, (1994). The assessment of bioaccumulation. Chemosphere 29: pp. 15011514 .

Hansch, C., Leo, A., and Hoekman, D. (1995). Exploring QSAR. Hydrophobic, Electronic, and Steric Constants. ACS Prof Ref Book. Washington, DC: American Chemical Society, p. 3 .

Hazardous Substance Database. (HSDB). http://toxnet.nlm.nih.gov/cgibin/sis/search/f?./temp/ PQGOJV:1.

Kane D.A and Williamson K.J. (1983). Arch Environment Contamination Toxicology 12: 447-53.

Lyman, WJ, WF Reehl, and DH Rosenblatt; (1990). Handbook of Chemical Property Estimation Methods. Washington, DC: American Chemical Society pp. 4-9, 15-1 to 15-29.

Meylan, W.M., Howard, P.H. and Boethling, R.S. (1992). Molecular topology/fragment contribution method for predicting soil sorption coefficients. Environmental Science and Technology 26: 1560-1567. 
Meylan WM, Howard PH, Boethling RS, Aronson D, Printup H, Gouchie S. (1999). Improved method for estimating bioconcetration/bioaccumulation factor from octanol/water partition coefficient. Environ Toxicol Chem. 18. 664-672.

Nwankwoala A.U, Egiebor N.O, Gilbert C \& Nyavor K (1999a); Microbial Degradation of Methylhydrazine Contaminated NASA Wastewater. In: American. Chemical. Society Div. Environ. Chem. Preprint Extend. Abstr., 217th ACS Nat. Meet., Vol. 39, PP. 43-44.

Nwankwoala A.U, Egiebor N.O, Gilbert C \& Nyavor K (1999b). Batch culture biodegradation of methylhydrazine contaminated NASA wastewater. Biodegradation 10: 105-112 (http://www.envirofacs.org/Preprints/Vol\%2042\%20No\%202/General/Papers\%20110-121/p\%20114.pdf)

O'Neil MJ, Merck Index, 13th ed, Whitehouse Station, NJ Merck \& Co. p 1086 (2001)

Perrin DD; (1965) Dissociation Constants of Organic Bases in Aqueous Solution. London: Butterworths.

Swann, R.L., Laskowski, D.A., McCall, P.J., van der Kuy, K., and Dishburger, H.J., (1983). A rapid method for estimation of the environmental parameters octanol/water partition coefficient, soil sorption constant water to air ratio and water solubility. Residue Rev. 85, pp. 17-28. (http://toxnet.nlm.nih.gov/cgi-bin/sis/search/r?dbs+hsdb:@term+@rn+6o$34-4)$.

Tuazon E.C., Carter W.P.L., Brown R.V, Atkinson R., Winer A.M. and Pitts J.N.Jr. (1982) Atmospheric reaction mechanisms of amine fuels. NTIS AD-A118267. http://oai.dtic.mil/oai/oai?verb=getRecord\&metadataPrefix=html\&identifier=ADA1182 67

Wachinski, A.M., Farmwald, J.A. (1979). The Toxicity and Biodegradability of Hydrazine Wastewaters Treated With UV-Chlorinolysis. Eng Serv Lab, Air Force Eng Serv Cent, (http://toxnet.nlm.nih.gov/cgi-bin/sis/search/r?dbs+hsdb:@term+@rn+6o-34-4) 


\section{Methyl Isocyanate 624-83-9}

\section{ENVIRONMENTAL TRANSPORT, DISTRIBUTION, AND TRANSFORMATION}

Air: According to a model of gas/particle partitioning of semivolatile organic compounds in the atmosphere (Bidleman 1988), methyl isocyanate, which has a vapor pressure of $348 \mathrm{~mm} \mathrm{Hg}$ at $20 \mathrm{deg}$ C (USEPA 1986), is expected to exist solely as a vapor in the ambient atmosphere. Vapor-phase methyl isocyanate is degraded in the atmosphere by reaction with photochemically-produced hydroxyl radicals; the half-life for this reaction in air is estimated to be 118 days, calculated from its rate constant of $1.4 \mathrm{X}_{10} \mathrm{O}^{-13} \mathrm{cu}$ $\mathrm{cm} /$ molecule-sec at $25 \mathrm{deg} \mathrm{C}$ (SRC), that was derived using a structure estimation method (Meylan and , Howard 1993). Methyl isocyanate does not contain chromophores that absorb at wavelengths $>290 \mathrm{~nm}$ and therefore is not expected to be susceptible to direct photolysis by sunlight (Lyman et al. 1994).

Water: If methyl isocyanate is released to water, it is expected to hydrolyze rapidly based on half-lives of $20 \mathrm{~min}$ at $15 \mathrm{deg} \mathrm{C}$ and $9 \mathrm{~min}$ at $25 \mathrm{deg} \mathrm{C}$, calculated from measured hydrolysis rate constants of 5.9X10-4 sec-1 and 1.34X10-3 $\mathrm{sec}^{-1}$, respectively (Castro et al. 1985). The products of hydrolysis may include $\mathrm{N}$-carboxymethylamine, methylamine, carbon dioxide, and N, N'-dimethylurea (Castro et al. 1985, USEPA 1986).

Bioconcentration, volatilization, adsorption to sediment and suspended solids, and biodegradation are not expected to be important fate processes since hydrolysis proceeds so quickly.

Soil: If methyl isocyanate is released to soil, it is expected to hydrolyze rapidly based on aqueous hydrolysis half-lives of $20 \mathrm{~min}$ at $15 \mathrm{deg} \mathrm{C}$ and $9 \mathrm{~min}$ at $25 \mathrm{deg} \mathrm{C}$, calculated from measured rate constants of 5.9X10-4 $\mathrm{sec}^{-1}$ and $1.34 \mathrm{X}^{-3} \mathrm{O}^{-3} \mathrm{sec}^{-1}$, respectively (Castro et al. 1985). Soil adsorption effects, volatilization, and biodegradation are not expected to be competing processes since hydrolysis proceeds so quickly. Methyl isocyanate is expected to volatilize from dry soil surfaces (SRC) based upon a vapor pressure of $348 \mathrm{~mm} \mathrm{Hg}$ (USEPA 1986).

Biota: Since methyl isocyanate hydrolyzes rapidly in water (Castro et al. 1985), biodegradation is not expected to be an important fate process.

\section{Regulatory}

MCL (Drinking Water): none 
Universal Treatment Standards (UTS): none

EPA Rf D: none

EPA RfC: none

Drinking Water Remedial Target: none

PRG: residential soil $\mathrm{mg} / \mathrm{kg}$ industrial soil $\mathrm{mg} / \mathrm{kg}$ ambient air $\mathrm{ug} / \mathrm{m} 3$ tap water $\mathrm{ug} / \mathrm{L}$ none none none none

\section{Remediation}

Since methyl isocyanate hydrolyzes rapidly in water (Castro et al. 1985), biodegradation is not expected to be an important fate process (SRC). Methyl isocyanate is expected to volatilize from dry soil surfaces (SRC) based upon an estimated vapor pressure of 348 $\mathrm{mm} \mathrm{Hg}$ at $20 \mathrm{deg} \mathrm{C}$ (USEPA 1986).

\section{References}

Bidleman, T. F. (1988) Atmospheric processes. Environmental Science and Technology. 22(4):361-367

Castro, E. A.; R. B. Moodie, P. J. Sansom. (1985) The Kinetics of Hydrolysis of Methyl and Phenyl Isocyanates. J. Chem. Soc., Perkin Trans. 2: 737-742.

Meylan, W.M and P.H. Howard. (1993) Computer estimation of the atmospheric gasphase reaction rate of organic compounds with hydroxyl radicals and ozone.

Chemosphere 26: 2293-99.

Lyman, W.J., W.F. Reehl and D.H. Rosenblatt, Editors (1990) Handbook of Chemical Property Estimation Methods, American Chemical Society, Washington D.C.

METHYL ISOCYANATE CASRN: 624-83-9 http://toxnet.nlm.nih.gov/cgi-bin/sis/search

USEPA, United States Environmental Protection Agency (1986) Health and Env Effects Profile for Methyl Isocyanate. Final Draft, ECAO-CIN-P217

http://www.epa.gov/ttn/uatw/hlthef/methylis.html 


\section{Monocrotophos 6923-22-4}

\section{ENVIRONMENTAL TRANSPORT, DISTRIBUTION, AND TRANSFORMATION}

Air: According to a model of gas/particle partitioning of semivolatile organic compounds in the atmosphere (Bidleman 1988), monocrotophos, which has a measured vapor pressure of $2.2 \mathrm{X}^{-6} \mathrm{O}^{-6} \mathrm{~mm} \mathrm{Hg}$ at $20 \mathrm{deg} \mathrm{C}$ (Tomlin 1994), is expected to exist both in the vapor and particulate phases in the ambient atmosphere. Vapor-phase monocrotophos is degraded in the atmosphere by reaction with photochemically-produced hydroxyl radicals and ozone (SRC); the half-lives for these reactions in air are estimated to be about 9.1 hours and 24 hours, respectively (Meylan and Howard 1993). Particulate-phase monocrotophos may be physically removed from the air by wet and dry deposition (SRC).

Water: An estimated Koc value of 19(SRC), determined from an experimental log Kow of -0.20 (Hansch et al. 1995), indicates that monocrotophos is not expected to adsorb to suspended solids and sediment in water (Swann et al. 1983). Monocrotophos is not expected to volatilize from water surfaces (Lyman et al.1990) based on an estimated Henry's Law constant of $6.5 \mathrm{X}^{-13} \mathrm{O}^{-13} \mathrm{~atm}-\mathrm{cu} \mathrm{m} / \mathrm{mole}(\mathrm{SRC})$, determined from experimental values for vapor pressure, $2.2 \mathrm{X}^{-6} \mathrm{O}^{-6} \mathrm{~mm} \mathrm{Hg}$ at $20 \mathrm{deg} \mathrm{C}$ (Tomlin 1994), and water solubility, $1.0 \mathrm{X}_{10^{6}} \mathrm{mg} / \mathrm{l}$ at $20 \mathrm{deg} \mathrm{C}$ (Shiu et al. 1990). According to a classification scheme (Shiu et al. 1990), an estimated BCF value of 0.41(Lyman et al.1990, SRC), from an experimental $\log$ Kow(2), suggests that bioconcentration in aquatic organisms is low(SRC). $100 \%$ of monocrotophos persisted after eight weeks incubation in river water (Eichelberger and Lichtenberg 1971).

Soil: An estimated Koc value of 19(SRC), determined from an experimental log Kow of 0.20 (Hansch et al. 1995) and a recommended regression-derived equation (Lyman et al. 1990), indicates that monocrotophos is expected to have very high mobility in soil (SRC) (Swann et al. 1983). Volatilization of monocrotophos from moist soil surfaces is not expected given an estimated Henry's Law constant of $6.5 \mathrm{X}^{-13} \mathrm{O}^{-13} \mathrm{~atm}-\mathrm{cu} \mathrm{m} / \mathrm{mole}(\mathrm{SRC})$, determined from experimental values for vapor pressure, $2.2 \mathrm{X}_{10} \mathrm{-}^{-6} \mathrm{~mm} \mathrm{Hg}$ at $20 \mathrm{deg} \mathrm{C}$ (Tomlin 1994), and water solubility, 1.0X106 $\mathrm{mg} / \mathrm{l}$ at $20 \mathrm{deg} \mathrm{C}$ (Shiu et al. 1990). Monocrotophos is not expected to volatilize from dry soil surfaces based on a measured vapor pressure of 2.2X10-6 mm Hg. Monocrotophos is rapidly degraded in soil with halflives ranging from 1 to 5 days (Tomlin 1994).

Biota: An estimated BCF value of 0.41 was calculated for monocrotophos, using an estimated log Kow of -0.20 (Hansch et al. 1995) and a recommended regression-derived 
equation (Lyman et al. 1990). According to a classification scheme (Franke et al. 1994), this BCF value suggests that bioconcentration in aquatic organisms is low.

\section{Environmental partitioning}

Photolysis of monocrotophos in soils, in water, and on bean plant leaves was investigated under sunlight and ultraviolet light. On a glass surface, percent recovery of monocrotophos in the dark was $98 \%$, while $72.8 \%$ was recovered after $8 \mathrm{hr}$ sunlight and $62 \%$ after UV light. Percent recovery of monocrotophos was 40.2 to $60.1 \%$ in soils after UV light exposure and 52.4 to $62.0 \%$ after sunlight exposure. Photodegradation in soils was of the order alluvial < black < red loamy < laterite. Percent recovery on bean leaves was $94 \%$ after $8 \mathrm{hr}$ darkness and $68 \%$ after $8 \mathrm{hr}$ sunlight. The rate of degradation of monocrotophos in tap water was approximately twice that in distilled water under sunlight (Dureja 1989).

\section{Regulatory}

MCL (Drinking Water): $1.0 \mathrm{mg} / \mathrm{L}$

Universal Treatment Standards (UTS): none

EPA Rf D: $0.0003 \mathrm{mg} / \mathrm{kg}$-day

EPA RfC: $0.0005 \mathrm{mg} / \mathrm{m} 3$

PRG: residential soil $\mathrm{mg} / \mathrm{kg}$ industrial soil $\mathrm{mg} / \mathrm{kg}$ ambient air $\mathrm{ug} / \mathrm{m}^{\wedge} 3$ tap water $\mathrm{ug} / \mathrm{l}$ none none none none

\section{Remediation}

Avoid skin contamination and inhalation of vapor. Cover contaminated areas and absorb spilled liquid with a 1:3 mixture of sodium carbonate crystals and damp sawdust, lime, sand, or earth. Sweep up and place in an impervious container. Ensure that container is tightly closed and labeled before transfer to a safe place for disposal (IPC).

Disposal: Large amounts should be incinerated at high temperature in a unit with effluent gas scrubbing. When no incinerator is available, bury in an approved dump, or in an area where there is no risk of contamination of surface or groundwater. Before burying, liberally mix with sodium carbonate (washing soda) crystals to help neutralize the product, and with soil rich in organic matter. Comply with any local legislation (IPC). 


\section{References}

Bidleman, T.F. (1988). Atmospheric processes: Great Lakes Pesticide Report .

Environmental Science and Technology. 22(4):361-367.

Dureja P. (1989). Photodecomposition of monocrotophos in soil, on plant foliage, and in water . Bull Environ Contam Toxicol 43: 239-45.

Eichelberger JW and Lichtenberg JJ. (1971). Persistence of. Pesticides in River Water. Environ Sci Technol 5: 541-4.

Franke, C., G. Studinger, G. Berger, S. Böhling, U. Bruckmann, D. Cohors-Fressenborg and U. Jöhncke. (1994). The assessment of bioaccumulation. Chemosphere 29:pp. 15011514.

Hansch, C., Leo, A., and Hoekman, D. (1995). Exploring QSAR. Hydrophobic, Electronic, and Steric Constants. ACS Prof Ref Book. Washington, DC: American Chemical Society, p. 3 .

Intenational Programme On Chemical Safety (IPCS). Health and Safety Guide No. 80.

Lyman, WJ, WF Reehl, and DH Rosenblatt. (1990). Handbook of Chemical Property Estimation Methods. Washington, DC: American Chemical Society pp. 4-9, 15-1 to 15-29.

Meylan, W.M. and Howard, P.H. (1991). Bond contribution method for estimating Henry's Law Constants. Environ. Toxicol. Chem. 10: 1283-93

Shiu, W. Y.; Ma, K. C.; Mackay, D.; Seiber, J. N.; Wauchope, R. D. (1990). Solubilities of pesticide chemicals in water part II: Data compilation. Rev Environ Contam Toxicol 116:15-187Rev. Environ. Contam. Toxicol. 1990, 116, 15-187.

Swann, R.L., Laskowski, D.A., McCall, P.J., van der Kuy, K., and Dishburger, H.J.. (1983). A rapid method for estimation of the environmental parameters octanol/water partition coefficient, soil sorption constant water to air ratio and water solubility. Residue Rev. 85, pp. 17-28. (http://toxnet.nlm.nih.gov/cgi-bin/sis/search/r?dbs+hsdb:@term+@rn+6o34-4).

Tomlin C. (1994). The Pesticide Manual. A World Compendium. Incorporating the Agrochemicals Handbook. 1oth ed. Bath, UK: The Bath Press. 


\section{Nicotine 54-11-5}

\section{ENVIRONMENTAL TRANSPORT, DISTRIBUTION, AND TRANSFORMATION}

Air: According to a model of gas/particle partitioning of semivolatile organic compounds in the atmosphere (Bidleman 1988), nicotine, which has a vapor pressure of $0.038 \mathrm{~mm}$ $\mathrm{Hg}$ at 25 deg C (Boublik et al. 1984), is expected to exist solely as a vapor in the ambient atmosphere. Vapor-phase nicotine is degraded in the atmosphere by reaction with photochemically-produced hydroxyl radicals (SRC); the half-life for this reaction in air is estimated to be 4 hours (Meylan and Howard 1993, SRC).

Soil: An estimated Koc value of $100($ SRC) determined from a log Kow value (Hansch et al. 1995) and a regression-derived equation (Lyman WJ et al. 1990), indicates that nicotine is expected to have high mobility in soil (SRC) (Swann et al. 1983). However, nicotine is a base, $\mathrm{pKb} 1=6.16 ; \mathrm{pKb} 2=10.96$ (Tomlin 1994), and protonation under neutral and acidic conditions may result in greater adsorption and less mobility than its estimated Koc or water solubility (Budavari 1996) indicate (Khairy AH et al. 1995, SRC). Volatilization of nicotine from moist soil surfaces is not expected to be important (SRC) given an estimated Henry's Law constant of 3.0X10-9 atm-cu m/mole (SRC), using a fragment constant estimation method. Nicotine is not expected to volatilize from dry soil surfaces based on a vapor pressure of $0.038 \mathrm{~mm} \mathrm{Hg}$ (Boublik $\mathrm{T}$ et al. 1994). Based on limited data, biodegradation of nicotine may occur with the formation of oxynicotine, $3^{-}$ pyridylmethyl ketone, 2,3'-dipyridyl, N-methylmyosmine and an unknown purple crystalline pigment as reaction products (Shulka 1984).

Sediment: Nicotine is not expected to adsorb to suspended solids and sediment in water (SRC) (Swann et al. 1984). However, nicotine is a base, pKb1=6.16; pKb2= 10.96(4), and protonation under neutral and acidic conditions may result in greater adsorption than its estimated Koc value may indicate (Khairy et al. 1990, SRC). Based on limited data, biodegradation of nicotine may occur with the formation of oxynicotine, $3^{-}$ pyridylmethyl ketone, 2, 3'-dipyridyl, N-methylmyosmine and an unknown purple crystalline pigment as reaction products (Shulka 1984). Nicotine is not expected to volatilize from water surfaces (Lyman et al. 1990,SRC) based on an estimated Henry's Law constant of 3.0X10-9 atm-cu m/mole(SRC), developed using a fragment constant estimation method (Meylan and Howard 1991).

Biota: According to a classification scheme by Franke et al. (1994), an estimated BCF of 5 (Lyman et al. 1990, SRC), from a log Kow value (Hansch et al. 1995), suggests the potential for bioconcentration in aquatic organisms is low. 


\section{Environmental partitioning}

Nicotine is a natural constituent of the tobacco plants Nicotiana tabacum and $\mathrm{N}$ rustica and it is released in the particulate phase of tobacco smoke. Nicotine may also be released to the environment due to its use as an insecticide and use in formulations for painting surfaces in poultry houses. If released to soil, nicotine may biodegrade to a variety of different products including oxynicotine and 3-pyridylmethyl ketone. Under alkaline conditions this compound should be extremely mobile. Nicotine is not expected to chemically hydrolyze or volatilize from moist soil surfaces. If released to water, nicotine may biodegrade. Slight potential also exists for photolysis. Chemical hydrolysis, bioaccumulation in the aquatic organisms, volatilization and adsorption under alkaline conditions to suspended solids and sediments are not expected to be significant fate processes. If released to air, nicotine is expected to degrade rather quickly in the presence of light and air. In the ambient atmosphere, nicotine may react with photochemically generated hydroxyl radicals (vapor phase half-life 1 day), be removed by wet deposition or photolyze.

\section{Regulatory}

IDLH/NIOSH: $5 \mathrm{mg} / \mathrm{m}^{3}$

MCL (Drinking Water): none

Universal Treatment Standards (UTS): none

EPA Rf D: none

EPA RfC: none

PRG: residential soil $\mathrm{mg} / \mathrm{kg}$ industrial soil $\mathrm{mg} / \mathrm{kg}$ ambient air $\mathrm{ug} / \mathrm{m}^{\wedge} 3$ tap water $\mathrm{ug} / \mathrm{I}$ none none none none

\section{Remediation}

Nicotine is a potential candidate for rotary kiln incineration at a temperature range of 820 to 1,600 deg $\mathrm{C}$ and residence times of seconds for liquids and gases, and hours for solids. It is also a potential candidate for fluidized bed incineration at a temperature range of 450 to 980 deg $\mathrm{C}$ and residence times of seconds for liquids and gases, and longer for solids (USEPA 1981). 
The most probable route of human exposure is by inhalation of tobacco smoke. Some people may also be exposed to nicotine in drinking water. Infants breast fed by women who smoke are exposed to nicotine in mother's milk. Worker exposure may occur during formulation, storage and application to pesticides and paints which contain this compound and during processing and extraction of tobacco (Spectrum).

\section{REFERENCES}

Bidleman, T.F. (1988) Atmospheric processes: Great Lakes Pesticide Report. Environmental Science and Technology. 22(4):361-367.

Boublik T. (1984) The Vapor Pressures of Pure Substances: Selected Values of the Temperature Dependence of the Vapour Pressures of Some Pure Substances in the Normal and Low Pressure Region. Vol. 17. Amsterdam, Netherlands: Elsevier Sci. Publ..

Budavari S. (1996) The Merck Index. 12th ed. Merck \& Co. Inc.: Whitehouse Station, NJ p. 1119.

Franke, C., G. Studinger, G. Berger, S. Böhling, U. Bruckmann, D. Cohors-Fressenborg and U. Jöhncke. (1994) The assessment of bioaccumulation. Chemosphere 29:1501-1514.

Hansch, C., Leo, A., and Hoekman, D. (1995) .Exploring QSAR. Hydrophobic, Electronic, and Steric Constants. ACS Prof Ref Book. Washington, DC: American Chemical Society, p. 3 .

Khairy,AH, Baghdadi,HH, and Ghabbour,EA. (1990) Adsorption mechanisma of nicotine on humic acid and clay humic acid complex, Z.Pflanzenernahr.Bodenk. 153, 33-38. www.mgumus.chem.msu.ru/referencesdb/au-id.php?id=1063.

Lyman, WJ, WF Reehl, and DH Rosenblatt; (1990) Handbook of Chemical Property Estimation Methods. Washington, DC: American Chemical Society pp. 4-9, 15-1 to 15-29.

Meylan, W.M. and Howard, P.H. (1991) Bond contribution method for estimating Henry's Law Constants. Environ. Toxicol. Chem. 10: 1283-93.

Meylan, W.M and P.H. Howard. (1993) Computer estimation of the atmospheric gasphase reaction rate of organic compounds with hydroxyl radicals and ozone.

Chemosphere 26: 2293-99. [AOPWIN (v1.84) computer estimation program available from Syracuse Research Corporation] 
Spectrum Laboratory Chemical Fact Sheet http://www.speclab.com/compound/c54115.htm. (Accessed Aug. 6, 2009).

Swann, R.L., Laskowski, D.A., McCall, P.J., van der Kuy, K., and Dishburger, H.J. (1983) A rapid method for estimation of the environmental parameters octanol/water partition coefficient, soil sorption constant water to air ratio and water solubility. Residue Rev. 85, pp. 17-28. (http://toxnet.nlm.nih.gov/cgi-bin/sis/search/r?dbs+hsdb:@term+@rn+6o34-4).

Tomlin C. (1994)T he Pesticide Manual. 1oth Ed, Surrey,UK: Crop Protection Pub, p. 735.

USEPA (United States Environmental Protection Agency). (1981) Engineering Handbook for Hazardous Waste Incineration p.3-9, EPA 68-03-3025. 


\title{
Nitric Acid
}

\section{ENVIRONMENTAL TRANSPORT, DISTRIBUTION, AND TRANSFORMATION}

\begin{abstract}
Air: In the atmosphere, nitric acid is present in very minute quantities as a gas or vapor. It reacts with ammonia in the atmosphere to form a nitrate compound that is unlikely to persist for more than 10 to 15 days. Nitric acid does not build up in plant or animal tissues largely because of its highly reactive properties. In a concentrated form, nitric acid reacts very quickly with many other commonly occurring natural or manufactured substances and compounds. In some cases this can produce a vigorous reaction in the form of a chemical fire or explosion (National Pollutant Inventory).
\end{abstract}

Water: Elevated nitrate levels will stimulate plankton and aquatic weed growth.

Soil: During transport through the soil, nitric acid will dissolve some of the soil material; in particular, the carbonate based materials. The acid will be neutralized to some degree with adsorption of the proton also occurring on clay materials. However, significant amounts of acid are expected to remain for transport down toward the ground water table. Upon reaching the ground water table, the acid will continue to move, now in the direction of the ground water flow. A contaminated plume will be produced with dilution and dispersion serving to reduce the acid concentration (Environment Canada).

Environmental Partitioning: Nitric acid exists in the atmosphere in the gas phase. The atmospheric removal processes for gaseous nitric acid are by wet and dry deposition. The estimated half-life and lifetime for dry deposition of nitric acid is 1.5 to 2 days and 2 to 3 days, respectively, and efficient rain out during episodic precipitation events. Nitric acid reacts with gaseous ammonia to form particulate or aerosol nitrate, which in turn is removed by wet and dry deposition of the particles. The average half-life and lifetime for particles in the atmosphere is about 3.5 to 10 days and 5 to 15 days (National Pollutant Inventory).

\section{Remediation}

Land spill: Dig a pit, pond, lagoon, or holding area to contain liquid or solid material. /SRP: If time permits; pits, ponds, lagoons, soak holes, or holding areas should be sealed with an impermeable flexible membrane liner. Dike surface flow using soil, sand bags, foamed polyurethane, or foamed concrete. Absorb bulk liquid with fly ash or cement powder. Neutralize with agricultural lime $(\mathrm{CaO})$, crushed limestone or sodium bicarbonate. 
Water spill: Neutralize with agricultural lime (slaked lime), crushed limestone, or sodium bicarbonate.

Air Release: Apply water spray or mist to knock down vapors. Vapor knockdown water is corrosive or toxic and should be diked for containment (Association of American Railroads).

\section{References}

Association of American Railroads. (1994) Emergency Handling of Hazardous Materials in Surface Transportation. Washington, DC: Association of American Railroads, Bureau of Explosives,. p.768.

Environment Canada (1978) Tech Info for Problem Spills: Nitric acid (Draft) p.71.

Mckee JE, Wolf HW (1978) Water Quality Criteria, 2nd Ed (1963) as cited in Environment Canada; Tech Info for Problem Spills: Nitric acid (Draft) p.82. National Pollutant Inventory http://www.npi.gov.au/database/substanceinfo/profiles/65.html (accessed August 6, 2009). 


\section{Nitrogen Dioxide 10102-44-o}

\section{ENVIRONMENTAL TRANSPORT, DISTRIBUTION, AND TRANSFORMATION}

Air: Nitrogen dioxide, which has a vapor pressure of $900 \mathrm{~mm} \mathrm{Hg}$ at $25 \mathrm{deg} \mathrm{C}$ (Daubert and Danner 1989), is expected to exist solely as a gas in the ambient atmosphere. Gasphase nitrogen dioxide is degraded in the atmosphere by reaction with photochemicallyproduced hydroxyl radicals (SRC); the half-life for this reaction in air is estimated to be 35 hours (SRC) from its rate constant of $1.1 \mathrm{X} 10-11 \mathrm{cu} \mathrm{cm} / \mathrm{molecule}-\mathrm{sec}$ at $25 \mathrm{deg} \mathrm{C}$ (Grosjean 1985). This compound is expected to undergo direct photolysis in the environment (SRC), ultimately leading to the production of ozone and smog conditions in the lower troposphere (Graham 1997).

Water: If released into water, nitrogen dioxide is expected to decompose (Graham et al. 1997) to nitric acid.

Soil: Nitrogen dioxide is expected to decompose to nitric acid in moist soil surfaces (Graham et al. 1997). The potential for volatilization of nitrogen dioxide from dry soil surfaces may exist based upon a vapor pressure of $900 \mathrm{~mm} \mathrm{Hg}$ at $25 \mathrm{deg} \mathrm{C}$ (Daubert and Danner 1989).

\section{Remediation}

Environmental considerations (air spill): Apply water spray or mist to knock down vapors. Vapor knockdown water is corrosive or toxic and should be diked for containment (Association of American Railroads).

\section{Regulatory}

EPA RfD: has been withdrawn from IRIS as of 09/01/1994

EPA RfC: none

IDLH: $20 \mathrm{ppb}$

$\begin{array}{cccc}\text { PRG: residential soil } \mathrm{mg} / \mathrm{kg} & \begin{array}{c}\text { industrial soil } \mathrm{mg} / \mathrm{kg} \\ \text { none }\end{array} & \begin{array}{c}\text { ambient air } \mathrm{ug} / \mathrm{m} 3 \\ \text { none }\end{array} & \text { tap water } \mathrm{ug} / \mathrm{L} \\ \text { none }\end{array}$ 


\section{References}

Association of American Railroads (1987) Emergency Handling of Hazardous Materials in Surface Transportation. Washington, D.C.: Assoc. of American Railroads, Hazardous Materials Systems (BOE), p. 495.

Daubert, TE and Danner, RP (1989) Physical and Thermodynamic Properties of Pure Chemicals Data Compilation Washington, DC: Taylor and Francis.

Graham J, Walker KD, Berry M, Bryan EFM, Callahan MA, Fan A, Finley B (1997) Environmental Health Criteria 188, WHO: Geneva, Switzerland.

Grosjean D. (1985) Atmospheric reactions of styrene and peroxybenzoyl nitrate. Sci Tot Environ. 46:41-59. 


\section{Nitroglycerine 55-63-0}

\section{ENVIRONMENTAL TRANSPORT, DISTRIBUTION, AND TRANSFORMATION}

Air: According to a model of gas/particle partitioning of semivolatile organic compounds in the atmosphere (Bidleman 1988), nitroglycerin, which has a vapor pressure of $2.0 \mathrm{X}_{10} \mathrm{O}^{-4}$ $\mathrm{mm} \mathrm{Hg}$ at $20 \mathrm{deg} \mathrm{C}$ (Lyman 1985), is expected to exist in both the vapor and particulate phases in the ambient atmosphere. Vapor-phase nitroglycerin is degraded in the atmosphere by reaction with photochemically-produced hydroxyl radicals (SRC); the half-life for this reaction in air is estimated to be 15 days (SRC), calculated from its rate constant of $1.1{\mathrm{X} 10^{-12}}^{-1 u} \mathrm{~cm} /$ molecule-sec at $25 \mathrm{deg} \mathrm{C}$ (SRC) that was derived using a structure estimation method (Meylan and Howard 1985). Particulate-phase nitroglycerin may be removed from the air by wet and dry deposition (SRC). Nitroglycerin absorbs light above $290 \mathrm{~nm}$ weakly and may be susceptible to direct photolysis (Spanggord et al. 1980).

Water: An estimated Koc value of 180 (SRC), determined from a log Kow of 1.62 (Hansch et al. 1995) and a regression-derived equation (Lyman et al. 1990), indicates that nitroglycerin is expected to adsorb to suspended solids and sediment (Swann et al. 1983). Volatilization from water surfaces is not expected (Lyman et al. 1990) based upon an estimated Henry's Law constant of $4.3 \mathrm{X}^{-8} \mathrm{O}^{-8} \mathrm{~atm}-\mathrm{cu} \mathrm{m} / \mathrm{mole}$. According to a classification scheme (Franke et al 1994), an estimated BCF of 4 (SRC), from its log Kow (Hansch et al. 1995) and a regression-derived equation (Meylan et al. 1999), suggests the potential for bioconcentration in aquatic organisms is low (SRC). Hydrolysis is expected to be an important environmental fate process under alkaline conditions based on half-lives of 37 and 96 days at pH 9 and 25 and 18 deg C, respectively (Cappellos et al. 1984).

Soil/ Sediment: An estimated Koc value of 180 (SRC), determined from a log Kow of 1.62 (Hansch et al. 1995) and a regression-derived equation (Lyman et al.1990), indicates that nitroglycerin is expected to have moderate mobility in soil (Swann et al. 1983), Volatilization of nitroglycerin from moist soil surfaces is not expected to be an important fate process (SRC) given an estimated Henry's Law constant of $4.3 \mathrm{X}_{10} \mathrm{O}^{-8} \mathrm{~atm}-\mathrm{cu} \mathrm{m} / \mathrm{mole}$ (SRC), derived from its vapor pressure, $2.0 \mathrm{X}_{10}^{-4} \mathrm{~mm} \mathrm{Hg}$, and water solubility, $1,38 \mathrm{o} \mathrm{mg} / \mathrm{l}$ (Seidell 1941). Nitroglycerin is not expected to volatilize from dry soil surfaces (SRC) based upon its vapor pressure. Hydrolysis under alkaline conditions may be important based upon half-lives of 37 and 96 days at pH 9 and 25 and $18 \mathrm{deg} \mathrm{C}$, respectively (Cappellos et al. 1984). Biodegradation of nitroglycerin occurs rapidly using batch reactors and digestors (Christodoulatos et al. 1997, Accashian et al. 2000), but its rate of biodegradation in soil is unknown (SRC). Nitroglycerin (10 ppm initial concentration) 
was completely biodegraded in 13 days using river water and river water/sediment microcosms obtained from a river near a munitions facility (Spanggord et al. 1980), which may suggest biodegradation can occur in soils (SRC).

Biota: According to a classification scheme (Franke et al 1994), an estimated BCF of 4 (SRC), from its log Kow (Hansch et al. 1995) and a regression-derived equation (Meylan et al. 1999), suggests the potential for bioconcentration in aquatic organisms is low (SRC).

\section{Regulatory}

Drinking Water Remedial Target: $48 \mu \mathrm{g} / \mathrm{l}$

DWEL (Drinking Water): 5 ug/l

Universal Treatment Standards (UTS): none

EPA RfD: $0.0001 \mathrm{mg} / \mathrm{kg}$-day (provisional)

EPA RfC: none $\mathrm{mg} / \mathrm{m}^{3}$

IDLH: $75 \mathrm{mg} / \mathrm{m}^{3}$

$\begin{array}{cccc}\text { PRG: residential soil mg/kg } & \text { industrial soil } \mathrm{mg} / \mathrm{kg} & \text { ambient air } \mathrm{ug} / \mathrm{m3} & \text { tap water } \mathrm{ug} / \mathrm{L} \\ 35 & 18 & 0.48 & 4.8\end{array}$

\section{Remediation}

Nitroglycerin and other aliphatic nitric acid esters undergo aerobic biodegradation readily via successive removal of nitrate groups to isomeric derivatives (Rendic $\mathrm{S}$ et al. 1995). Contrary to some earlier reports that it was recalcitrant to biodegradation, nitroglycerin proved to be readily biodegradable in batch and continuous tests (Wendt et al. 1978). Results of the shake flask test that was run at 30 deg $C$ with an activated sludge inoculum showed a $53.6 \%$ loss of nitroglycerin in 5 days (Wendt et al. 1978). In a continuous bench-scale activated sludge apparatus, a mean reduction of $92.2 \%$ was obtained (Wendt et al. 1978). The apparatus was run at room temperature and the residence time was $84 \mathrm{hr}$. 1, 3-Dinitroglycerol and 1,2-dinitroglycerol were identified at intermediate stages of the process, but they were also essentially absent from the effluent (Wendt et al. 1978). The third experiment employing a chemostat, a continuous culture, aerobic system with no solids recycling, was designed to simulate a plant where propellant wastes would be treated. After a 8-15 hr detention period, no nitrate esters 
were detectable in the effluent (Wendt et al. 1978). It was found that nitroglycerin is not suitable as a source of carbon and nitrogen so nutrients are essential (Wendt et al. 1978). It was speculated that the earlier experiments which showed nitroglycerin to be recalcitrant were conducted using concentrations of nitroglycerin that were toxic to the microorganisms (Wendt et al. 1978).

Complete mineralization of nitroglycerin (350 umol/l initial concentration) was achieved within 4 days utilizing mixed cultures and an anaerobic digester, using digester sludge from a municipal wastewater treatment plant amended with 2,000 $\mathrm{mg} / \mathrm{l}$ of glucose (Christodoulatos et al.1997). Without glucose amendments, the complete mineralization of nitroglycerin took about 25 days in the digester (Christodoulatos et al.1997). Nitroglycerin, present at $16 \mathrm{mg} / \mathrm{l}$, was rapidly degraded in a sequencing batch reactor using mixed liquor from an industrial wastewater facility and a domestic wastewater treatment plant, with 1,2-dinitroglycerol and 1,3-dinitroglycerol as the initial products (Accashian JV et al.2000).

Nitroglycerin (10 ppm initial concentration) was completely biodegraded in 13 days using river water and river water/sediment microcosms obtained from a river near a munitions facility in Virginia. Pure cultures isolated from river water and sediment near a munitions factory in Virginia degraded nitroglycerin. The first-order rate constant was 0.60 hours-1, which corresponds to a half-life of about 1 hour (Spanggord et al.1995).

\section{References}

Accashian, J. V., B. F. Smets, and B. J. Kim.(2000) Aerobic biodegradation of nitroglycerin in a sequencing batch reactor. Water Environment. Research 72:499-506.

Bidleman, T.F. (1988) Atmospheric processes: Great Lakes Pesticide Report Environmental Science and Technology. 22(4):361-367.

Cappellos, C. et al (1984) Int J Chem Kinet 16: 1027-51 (cited in Hazardous Substances Data Bank http://toxnet.nlm.nih.gov (accessed August 2009)).

Christodoulatos C, Bhaumik S, Brodman B W. (1997) Anaerobic biodegration of nitroglycerin. Water Res. 31:1462-1470.

Franke, C., G. Studinger, G. Berger, S. Böhling, U. Bruckmann, D. Cohors-Fressenborg and U. Jöhncke. (1994) The assessment of bioaccumulation. Chemosphere 29: 1501-14. 
Hansch C,, Leo, A., and Hoekman, D. (1995) Exploring QSAR. Hydrophobic, Electronic, and Steric Constants. ACS Prof Ref Book. Heller SR, consult. ed., Washington, DC: Amer Chem Soc p. 6.

Lyman WJ. (1985) Environmental Exposure From Chemicals Vol I, p. 31 Neely WB, Blau GE, eds, Boca Raton, FL: CRC Press.

Lyman, WJ, WF Reehl, and DH Rosenblatt. (1990) Handbook of Chemical Property Estimation Methods. Washington, DC: Amer Chem Soc pp. 4-9, 15-1 to 15-29.

Meylan, W. M. and P. H. Howard. (1993) Reliability: Estimate based on known qualitative structure-activity relationships Chemosphere, 26:2293-99.

Meylan, WM, Howard, PH, Boethling, RS, Aronson, D, Printup, H, Gouchie, S. (1999) Improved method for estimating bioconcentration/bioaccumulation factor from octanol/water partition coefficient. Environ Toxicol Chem 18(4):664-72.

Seidell, A.(1941) Solubilities of Organic Compounds. NY, NY D Van Norstand Co, Inc.

Spanggord, R.J; Mill, T; Chou, T; Mabey, W.R.; Smith, J.H. (1980) Environmental Fate Studies on Certain Munition Wastewater Constituents. Final Report, Phase II Laboratory Studies. NTIS A099256, US Army Med Res Devel, Fredrick, MD.

Swann, R.L., Laskowski, D.A., McCall, P.J., van der Kuy, K., and Dishburger, H.J. (1983) A rapid method for estimation of the environmental parameters octanol/water partition coefficient, soil sorption constant water to air ratio and water solubility. Residue Rev. 85, pp. 17-28. (http://toxnet.nlm.nih.gov/cgi-bin/sis/search/r?dbs+hsdb:@term+@rn+6o34-4).

Rendić, S., Jurišić, B., and Medić-Šarić, M.(1995) Biological and Environmental Properties of nitro-, nitramine-, and nitrate compounds: Explosives and Drugs. Environmental Toxicology Assessment (M.L. Richardson, Ed.) Taylor \& Francis, London, pp. 303-315.

Wendt, TM, Cornell, JH, Kaplan. AM. (1978) Microbial degradation of glycerol nitrates. Appl Environ Microbiol 36: 693-9. 


\title{
Parathion 56-38-2
}

\section{ENVIRONMENTAL TRANSPORT, DISTRIBUTION, AND TRANSFORMATION}

\begin{abstract}
Air: According to a model of gas/particle partitioning of semivolatile organic compounds in the atmosphere (Bidleman 1988), parathion, which has a vapor pressure of $6.68 \mathrm{X}^{-6} \mathrm{O}^{-6}$ $\mathrm{mm} \mathrm{Hg}$ at $20 \mathrm{deg} \mathrm{C}$ (Tomlin 1997), will exist in both the vapor and particulate phases in the ambient atmosphere. Vapor-phase parathion is degraded in the atmosphere by reaction with photochemically-produced hydroxyl radicals (SRC); the half-life for this reaction in air is estimated to be 4.2 hours (SRC) from the estimated rate constant (Meylan and Howard 1993). Particulate-phase parathion may be removed from the air by wet and dry deposition (SRC). Parathion absorbs radiation with wavelengths shorter than $320 \mathrm{~nm}$, suggesting a potential for direct photolysis (SRC).
\end{abstract}

Water: Based on a classification scheme (Swann et al. 1983), sediment Koc values ranging from 3,086 to 38,000 (House et al. 1992) indicate that some adsorption of parathion to suspended solids and sediment in the water column is expected (SRC). Parathion is not expected to volatilize from water surfaces (Lyman et al. 1990) based upon a Henry's Law constant of $2.98 \times 10^{-7}$ atm-cu m/mole (Tomlin 1997). According to a classification scheme (Franke et al. 1994), BCFs ranging from 63 to 462 (USEPA), suggest bioconcentration in aquatic organisms is moderate to high (SRC). Irradiation of parathion for $10 \mathrm{hr}$ in aerated distilled water resulted in $88 \%$ degradation (Mansour et al. 1983). $20 \%$ of parathion was lost by photolysis in $2 \mathrm{hr}$ in Okeefenokee Swamp water (Zepp and Baughman 1978). Reported hydrolysis half-lives at $20 \mathrm{deg} \mathrm{C}$ at environmentally relevant pHs range from 3 weeks at pH 9(Faust and Gomaa 1972) to 43 weeks at $\mathrm{pH} 5$; the half-life for hydrolysis in sterile sea water has been reported to be approximately $1 \mathrm{yr}$ at $4 \mathrm{deg} \mathrm{C}$. It is thought that divalent cations catalyze hydrolysis (Wade 1979). Parathion biodegrades in acclimated natural waters within several weeks (Eichelberger and Lichtenberg 1971). Parathion, 5 ppm, completely degraded within 2 weeks in acclimated water from Holland Marsh, Ontario, being almost quantitatively converted to aminoparathion; only 10\% degradation occurred in 16 weeks when the water was sterilized (Sharom 1980). After 30 days incubation in non-sterile (sterile) coastal river water, only 21,14 , and $6 \%(64,57$, and $49 \%)$ of parathion remained at $\mathrm{pH}$ values of 6, 7, and 8.16, respectively (Wang and Hoffman 1991).

Soil: Persistence of parathion was partially dependent on soil type. In some soils degradation was rapid \& probably through combination of hydrolysis \& strong microbial activity. In other soils, loss was slow \& attributable to hydrolysis (Menzie 1978). Based on a classification scheme (Swann et al. 1983), Koc values ranging from 314 to 15,860 
(Mingelgrin and Gerstl 1983) indicate that parathion is expected to have moderate to no mobility in soil (SRC). Volatilization of parathion from moist soil surfaces is not expected to be an important fate process (SRC) given a Henry's Law constant of $2.98 \times 10^{-7} \mathrm{~atm}-\mathrm{cu}$ $\mathrm{m} /$ mole (Tomlin 1997). Parathion is not expected to volatilize from dry soil surfaces based upon a vapor pressure of $6.68 \times 10^{-6} \mathrm{~mm} \mathrm{Hg}$ at $20 \mathrm{deg} \mathrm{C}$ (Tomlin 1997). The half-life for photodecomposition of parathion on 3 soils with various water contents ranged from 31 to 70 hr (Hautala 1978). After 8 weeks of incubation in an organic and a mineral soil, $<2$ and $6 \%$, respectively, of the 1 ppm parathion applied remained; in sterilized controls 80 and 95\% remained(Chapman et al. 1981). Prior exposure of soils to p-nitrophenol resulted in increased mineralization of parathion to carbon dioxide (Somasundaram and Coats 1990). Metabolic pathways involve both oxidative and reductive reactions. The primary oxidative pathway involves an initial hydrolysis to p-nitrophenol and diethylthiophosphoric acid; a second oxidative pathway involves oxidation to paraoxon. Under low oxygen conditions reduction to aminoparathion occurs (Miles et al. 1979).

Biota: Tadpoles, a species resistant to cholinesterase inhibitors such as organophosphate pesticides, had an average bioconcentration factor of 64(Hall and Kolbe 1980). BCFs ranging from 63 to 462 were observed in bluegill after 0.5 and 3 days exposure to parathion concentrations of 510 and $640 \mathrm{ug} / \mathrm{l}$, respectively (USEPA 1998). BCFs ranging from 68 to 344 were observed in brook trout after 0.33 and 5.83 days exposure to parathion concentrations of 3,180 and $270 \mathrm{ug} / \mathrm{l}$, respectively (USEPA 1998). In a terrestrial-aquatic ecosystem, there was no evidence for bioaccumulation in any of the organisms, including snails, algae, daphnia, mosquito larvae, and fish, at the end of the 38-day experiment, although the ecosystem organisms contained radiolabeled carbon indicating the presence of metabolism products (Sanborn et al. 1977). There is no evidence of bioaccumulated parathion in cattle, sheep, or rabbits (Sanborn et al. 1977). The average BCF in killifish after 24 to 72 hours exposure to the individual pesticide was 98; the average BCF was 88 in killifish after 24 to 72 hours exposure to a mixture of pesticides, including parathion (Tsuda et al. 1995). According to a classification scheme (Franke et al. 1990), these BCFs suggest bioconcentration in aquatic organisms is moderate to high.

\section{Regulatory}

MCL (Drinking Water): none

Universal Treatment Standards (UTS): $0.014 \mathrm{mg} / \mathrm{l}^{2}$ in wastewater standards

EPA RfD: $0.006 \mathrm{mg} / \mathrm{kg}$-day (provisional) 
EPA RfC: none $\mathrm{mg} / \mathrm{m}^{3}$

IDLH: $10 \mathrm{mg} / \mathrm{m}^{3}$

PRG: residential soil mg/kg industrial soil $\mathrm{mg} / \mathrm{kg}$ ambient air ug/m3 tap water $\mathrm{ug} / \mathrm{L}$ 370

5300

22

220

\section{Remediation}

Land spill: Dig a pit, pond, lagoon, holding area to contain liquid or solid material. SRP: If time permits, pits, ponds, lagoons, soak holes, or holding areas should be sealed with an impermeable flexible membrane liner. Dike surface flow using soil, sand bags, foamed polyurethane, or foamed concrete. Absorb bulk liquid with fly ash, cement powder, or commercial sorbents. Apply "universal" gelling agent to immobilize spill. Parathion and compressed gas mixture (Association of American Railroads).

Water spill: Use natural barriers or oil spill control booms to limit spill travel. Use surface active agent (eg, detergent, soaps, alcohols), if approved by EPA. Inject "universal" gelling agent to solidify encircled spill and increase effectiveness of booms. If dissolved, in region of $10 \mathrm{ppm}$ or greater concentration, apply activated carbon at ten times the spilled amount. Use mechanical dredges or lifts to remove immobilized masses of pollutants and precipitates. Parathion and compressed gas mixture (Association of American Railroads).

\section{References}

Association of American Railroads. (1994) Emergency Handling of Hazardous Materials in Surface Transportation. Washington, DC: Association of American Railroads, Bureau of Explosives, p. 827.

Bidleman, T.F. (1988) Atmospheric processes: Great Lakes Pesticide Report, Environmental Science and Technology. 22(4):361-367.

Chapman RA, Tu CM, Harris CR, Cole C. (1981) "Persistence of five pyrethroid insecticides in sterile and natural, mineral and organic soil.” Bull Environ Contam Toxicol 26: 513-9.

Chapman RA and Cole CM (1982) Observations on the influence of water and soil. $\mathrm{pH}$ on the persistence of insecticides. J Environ Sci Health B17: 487-504.

Eichelberger, J. W., Lichtenberg, J. J.(1971) "Persistence of. Pesticides in River Water," Environ. Soi. Teohnol., 5:541-4. 
Faust, S. D.; Gomaa, H. M. (1972) Chemical Hydrolysis of Some Organic Phosphorus and Carbamate Pesticides in Aquatic Environments. Environ. Lett. 3:171-201.

Franke, C., G. Studinger, G. Berger, S. Böhling, U. Bruckmann, D. Cohors-Fressenborg and U. Jöhncke (1994). The assessment of bioaccumulation. Chemosphere 29: 1501-14..

Hall, R. J. and E. Kolbe (1980) Bioconcentration of organophosphorus pesticides to hazardous levels by amphibians. Journal of Toxicology and Environmental Health 6:853860 .

Hautala, RP (1978) Surfactant Effects on Pestic Photochem in Water and Soil. USEPA600/3-78-060.

House, W.A., J.E. Rae and R.T. Kimblin (1992) Source-sediment controls on the riverine transport of pesticides. In: B.B.C.P. Council, Editor, Brighton Crop Protection Conference: Pests and deseases, International Conference Brighton England UK, BCPC British Crop Protection Council, Farnham, UK 1:865-870.

Lyman WJ et al (1990) Handbook of Chemical Property Estimation Methods. Washington,DC: Amer Chem Soc pp. 4-9, 5-4, 5-10, 15-1 to 15-29.

Mansour M, Thaller S, Korte F. (1983) Action of sunlight on parathion, Bull Environ Contam Toxicol 30: 358-64.

Menzie, C.M. (1978) Metabolism of Pesticides, Update II. U.S. Department of the Interior, Fish Wildlife Service, Special Scientific Report - Wildlife No. 2l2. Washington, DC: U.S. Government Printing Office p. 204.

Meylan, W. M. and P. H. Howard (1993) Reliability: Estimate based on known qualitative structure-activity relationships Chemosphere, 26:2293-99.

Miles JRW, Tu CM, Harris CR (1979) Persistence of eight organophosphate insecticides in sterile and non-sterile mineral and organic soils. Bull Environ Contam Toxicol 22:312318.

Mingelgrin, U. and Z. Gerstl (1983) Reevaluation of Partitioning as a Mechanism of Nonionic Chemicals Adsorption in Soils J Environ Qual 12: 1-11.

Sanborn, J.R., Francis, B.M., and Metcalf, R.L. (1977) The degradation of selected pesticides in soil. EPA 600/9-77-022. pp. 573-582 
Sharom MS, Miles JRW, Harris CR, et al. (1980) Behaviour of 12 insecticides in soil and aqueous suspensions of soil and sediment. Water Res 14:1089-93.

Somasundaram, L and Coats, JR (1990) Enhanced Biodegradation of Pesticides in the Environment. ACS Symp Ser 426: 128-40.

Swann, R.L., Laskowski, D.A., McCall, P.J., van der Kuy, K. and Dishburger, H.J. (1983) A rapid method for estimation of the environmental parameters octanol/water partition coefficient, soil sorption constant water to air ratio and water solubility. Residue Rev. 85, pp. 17-28. (http://toxnet.nlm.nih.gov/cgi-bin/sis/search/r?dbs+hsdb:@term+@rn+60-34-4).

Tomlin C. (1997) The Pesticide Manual World Compendium 11th ed., Surrey, England: British Crop Protection Council p. 965.

Tsuda T, S. Aokia, T. Inoue, and M. Kojima (1995) Accumulation of IBP, parathion and EPN by killifish: comparison of individual and mixed pesticides , Comparative Biochemistry and Physiology Part C: Pharmacology, Toxicology and Endocrinology 111(1):19-22

USEPA (1998) Chemical Information System, IS Aquire Data Base.

Wade MJ. (1979) Organophosphorus pesticides in the marine environment: Their transport and fate. Dissertation Abstracts International 40:4704.

Wang, T. C.and Hoffman, M. E. (1991) Degradation of organophosphorus pesticides in coastal water. J. Assoc. Off. Anal. Chem. 74:883-886.

Zepp RG and Baughman GL (1978) Prediction of photochemical transfer nature of pollutants in the aquatic environment. Aquatic Pollut (Repr), Hutzinger O, Van Lelyveld IH, Zoeteman BCJ, eds, Permagon Press. 


\section{Phenol 108-95-2}

\section{ENVIRONMENTAL TRANSPORT, DISTRIBUTION, AND TRANSFORMATION}

Air: The gas-phase reaction of phenol with photochemically produced hydroxyl radicals is probably a major removal mechanism in the atmosphere. An estimated half-life for phenol for this reaction is 0.61 days (EPA 1979a). The reaction of phenol with nitrate radicals during the night may constitute a significant removal process. This is based on a rate constant of $3.8 \times 10^{-12} \mathrm{~cm} 3 /$ molecule second for this reaction, corresponding to a halflife of 15 minutes at an atmospheric concentration of $2 \times 10^{8}$ nitrate radicals per $\mathrm{cm}^{3}$ (Atkinson et al. 1987). The reaction of phenol with nitrate radicals present in the atmosphere during smog episodes may decrease the half-life of phenol in polluted atmospheres. The above data indicate that phenol has a short half-life in the atmosphere, probably <1 day. Phenol does not absorb light in the region of $290-330 \mathrm{~nm}$ (Lide and Milne 1994); therefore, it should not photo-degrade directly in the atmosphere.

Water: Although phenol does not absorb light at wavelengths $>290$, phenols react rapidly to sunlit natural water via an indirect reaction with photochemically produced hydroxyl radicals and peroxyl radicals; typical half-lives for hydroxyl and peroxyl radical reactions are on the order of 100 and 19.2 hours of sunlight, respectively (Canonica et al. 1995; Mill and Mabey 1985). These reactions require dissolved natural organic materials that function as photosensitizers (Canonica et al. 1995). The estimated half-life for the reaction of phenol with photochemically produced singlet oxygen in sunlit surface waters contaminated by humic substances is 83 days (assuming Switzerland summer sunlight and a singlet oxygen concentration of $4 \mathrm{X1O}^{-14}$ molar [M]) (Scully and Hoigne 1987). Phenol is readily biodegradable in natural water, provided the concentration is not high enough to cause significant inhibition through microbial toxicity. Complete degradation in $<1$ day has been reported in water from three lakes; the rates of degradation were affected by the concentration of organic and inorganic nutrients in the water (Rubin and Alexander 1983). Complete removal of phenol in river water has been reported after 2 days at $20{ }^{\circ} \mathrm{C}$ and after 4 days at $4{ }^{\circ} \mathrm{C}$ (Ludzack and Ettinger 1960). The degradation of phenol is somewhat slower in salt water, and a half-life of 9 days has been reported in an estuarine river (EPA 1979b).

Sediment and Soil: Available data indicate that phenol biodegrades in soil under both aerobic and anaerobic soil conditions. The half-life of phenol in soil is generally $<5$ days (Baker and Mayfield 1980), but acidic soils and some surface soils may have half-lives of up to 23 days (Shiu et al. 1994). Mineralization in an alkaline, parabrown soil under aerobic conditions was $45.5,48$, and $65 \%$ after 3 , 7, and 70 days, respectively (Haider et 
al. 1974). Half-lives for degradation of low concentrations of phenol in two silt loam soils were 2.70 and 3.51 hours (Scott et al. 1983). Plants have been shown to be capable of metabolizing phenol readily (DOE 1987). While degradation is slower under anaerobic conditions, evidence presented in the literature suggests that phenol can be rapidly and virtually completely degraded in soil under both aerobic and anaerobic conditions (Baker and Mayfield 1980).

Biota: Phenol is not expected to bioconcentrate significantly in aquatic organisms. Reported $\log$ bioconcentration factors (BCF) in fish for phenol include 0.28 for goldfish, (Kobayashi et al. 1979) and 1.3 for golden orfe (Freitag et al. 1984). The highest mean level of phenol detected in bottom fish from Commencement Bay in Tacoma, Washington, was 0.14 ppm (Nicola et al. 1987). The levels of phenol in the water or sediments were not stated.

\section{Environmental partitioning}

Phenol is released into the air and discharged into water from both manufacturing and use. Based on its high water solubility and the fact that it has been detected in rainwater, some phenol may wash out of the atmosphere; however, it is probable that only limited amounts wash out because of the short atmospheric half-life of phenol. During the day, when photochemically produced hydroxyl radical concentrations are highest in the atmosphere, very little atmospheric transport of phenol is likely to occur.

In water, neither volatilization nor sorption to sediments and suspended particulates are expected to be important transport mechanisms. Using the Henry's Law constant of $3 \times 10^{-}$ 7 atm m3/mol (Gaffney et al. 1987), a volatilization half-life of 88 days was calculated for phenol evaporation from a model river $1 \mathrm{~m}$ deep with a current of $1 \mathrm{~m} /$ second, and wind velocity of $3 \mathrm{~m} /$ second (Lyman et al. 1982). The biological treatment of waste water containing phenol has shown that $<1 \%$ of phenol is removed by stripping (Kincannon et al. 1983; Petrasek et al. 1983).

The pKa of phenol is 10 (O’Neil 2001), indicating that phenol will primarily exist as the protonated acid at environmental $\mathrm{pH}$ values. In alkaline soils and water, phenol will partially exist as an anion, which can affect its fate and transport processes. Although it has been shown that plants readily uptake phenol (DOE 1987), bioaccumulation does not take place due to a high rate of respiratory decomposition of phenol to $\mathrm{CO} 2$.

\section{Regulatory}

MCL (Drinking Water): $0.3 \mathrm{mg} / \mathrm{L}$ cyanide 
Universal Treatment Standards (UTS): $0.039 \mathrm{mg} / \mathrm{l}^{2}$ in wastewater standard

$6.2 \mathrm{mg} / \mathrm{L}$ in nonwastewater standard

EPA RfD: $0.6 \mathrm{mg} / \mathrm{kg}$-day

EPA RfC: $0.006 \mathrm{mg} / \mathrm{m}^{3}$

PRG: residential soil $\mathrm{mg} / \mathrm{kg}$ industrial soil $\mathrm{mg} / \mathrm{kg}$ ambient air $\mathrm{ug} / \mathrm{m}^{\wedge} 3$ tap water $\mathrm{ug} / \mathrm{l}$ $37000 \quad 10000 \quad 2200 \quad 22000$

\section{Remediation}

Phenol is quickly broken down in the air, usually within 1-2 days.

Phenol may persist in water for a week or more.

Phenol that remains in soil may be broken down by bacteria or other microorganisms.

\section{References}

Atkinson R, Aschmann SM, Winer AM (1987) Kinetics of the reactions of NO3 radicals with a series of aromatic compounds. Environ Sci Technol 21:1123-1126.

Baker MD, Mayfield CI (1980) Microbial and nonbiological decomposition of chlorophenols and phenol in soil. Water Air Soil Pollut 13:411-424.

Canonica S, Jans U, Stemmler K, et al. (1995) Transformation kinetics of phenols in water: Photosensitization by dissolved natural organic material and aromatic ketones. Environ Sci Technol 29:1822-1831.

DOE. (1987) Uptake and fate of phenol aniline and quinoline in terrestrial plants. In: Gray RH, ed. Health and environmental research on complex organic mixtures. U.S. Department of Energy, 631-641.

EPA. (1979a) Atmospheric reaction products of organic compounds. Washington, DC: U.S. Environmental Protection Agency, Office of Toxic Substances. EPA5601279001. PB301384.

EPA (1979b) Microbial degradation of organochlorine compounds in estuarine waters and sediments. In: Proceedings Workshop: Microbial Degradation Pollution Marine 
Environment, 1978. Washington, DC: U.S. Environmental Protection Agency, Office of Research and Development. EPA600979012.

Freitag D, Lay JP, Korte F (1984) Environmental hazard -Test results as related to structures and transplantation into the environment. In: Kaiser, KLE, ed. Quantitative Structure Activity Relationships Environmental Toxicology Proceedings Workshop. Boston, MA: D. Reidel Publishing Co., 111-136.

Gaffney JS, Streit GE, Spall WD, et al. (1987) Beyond acid rain: Do soluble oxidants and organic toxins interact with $\mathrm{SO}_{2}$ and $\mathrm{NO}_{x}$ to increase ecosystem effects? Environ Sci Technol 21:519-524.

Haider K, Jagnow G, Kohnen R, et al. (1974) [Degradation of chlorinated benzol, phenol and cyclohexane derivatives by soil bacteria that utilize benzol and phenol under aerobic conditions.] Arch Microbiol 96:183-200. (German)

Kincannon DF, Stover EL, Nichols V, et al. (1983) Removal mechanisms for toxic priority pollutants. J Water Pollut Control Fed 55:157-163.

Kobayashi K, Akitake H, Manadge K. (1979) Relation between toxicity and accumulation of various chlorophenols in goldfish. Bull Jap Soc Sci Fish 45:173-175.

Lide DR and Milne GWA. (1994) Phenol. In: Handbook of data on organic compounds. Boca Raton, FL: CRC Press, 3990.

Ludzack FJ and Ettinger MB. (1960) Chemical structures resistant to aerobic biochemical stabilization. J Water Pollut Control Fed 32:1173-1200.

Lyman WJ, Reehl WF, and Rosenblatt DH. (1982) Handbook of chemical property estimation methods. Environmental behavior of organic compounds. New York, NY: McGraw-Hill Book Co., pp. 15.9-15.31.

Mill Tand Mabey W (1985) Photochemical transformations. In: Neely WB, Blau GE, eds. Environmental exposure from chemicals, Boca Raton, FL: CRC Press, 1:175-216.

Nicola RM, Branchflower R, and Pierce D (1987) Chemical contaminants in bottomfish. J Environ Health 49:342-347.

O’Neil MJ (2001) Phenol. In: Merck index: An encyclopedia of chemicals, drugs, and biologicals. Whitehouse Station, NJ: Merck Research Laboratories, 1299-1300.

Petrasek AC, Kugelman IJ, Austern BM, et al. (1983) Fate of toxic organic compounds in wastewater treatment plants. J Water Pollut Control Fed 55:1286-1296. 
Rubin HE, Alexander M. (1983) Effect of nutrients on the rates of mineralization of trace concentrations of phenol and p-nitrophenol. Environ Sci Technol 17:104-107.

Scott HD, Wolf DC, Lavy TL. (1983) Adsorption and degradation of phenol at low concentrations in soil. J Environ Qual 12:91-95.

Scully FE, Jr, Hoigne JR. (1987) Rate constants for reactions of singlet oxygen with phenols and other compounds in water. Chemosphere 16:681-694.

Shiu WY, Ma KC, Varhanickova AD, et al. (1994) Chlorophenols and alkylphenols: A review and correlation of environmentally relevant properties and fate in an evaluative environment. Chemosphere 29:1155-1224. 


\section{Phosgene 75-44-5}

\section{ENVIRONMENTAL TRANSPORT, DISTRIBUTION, AND TRANSFORMATION}

Air: According to a model of gas/particle partitioning of semivolatile organic compounds in the atmosphere (Bidleman 1988), phosgene, which has a vapor pressure of $1420 \mathrm{~mm}$ $\mathrm{Hg}$ at 25 deg C (Daubert and Danner 1989), is expected to exist solely in the gas phase in the ambient atmosphere. Gas-phase phosgene is degraded in the atmosphere by reaction with photochemically-produced hydroxyl radicals (SRC); the half-life for this reaction in air is estimated to be 44 years (SRC), calculated from its rate constant of $1.0 \mathrm{X}^{-10^{-15} \mathrm{cu}}$ $\mathrm{cm} /$ molecule-sec at 25 deg C (Atkinson 1994). Phosgene does not absorb UV radiation at wavelengths $>290 \mathrm{~nm}$ (Montgomery and Rollefson 1993) and therefore would not be subject to direct photolysis by sunlight in the troposphere. Phosgene's hydrolytic half-life in the atmosphere, extrapolating from high temperature data, is $113 \mathrm{yr}$ at sea level, assuming 10 torr of water vapor (Butler et al 1979).

Water: Based on a classification scheme (Swann et al 1983), an estimated Koc value of 2.2 (SRC), determined from a structure estimation method (Meylan et al 1992), indicates that phosgene is not expected to adsorb to suspended solids and sediment (SRC).

Volatilization from water surfaces is expected (Lyman et al 1990) based upon a Henry's Law constant of 1.7X10-2 atm-cu m/mole (De Bruyn et al 1995). Phosgene has been reported to hydrolyze rapidly in seawater (Talmage et al 2007). Hydrolysis of a $1 \%$ solution of phosgene in water is complete within 20 seconds at o deg C (Mitretek Systems 2000). Measured aqueous phase hydrolysis rate constants for phosgene have ranged from 0.29 to $100 \mathrm{sec}^{-1}$ (Kolb et al 1995). The rapid hydrolysis suggests that bioconcentration will not be an important environmental fate process. Biodegradation data were not available (SRC, 2007).

Soil/Sediment: Based on a classification scheme (Swann et al 1983), an estimated Koc value of 2.2, determined from a structure estimation method (Meylan et al 1992), indicates that phosgene is expected to have very high mobility in soil (SRC). Volatilization of phosgene from moist soil surfaces is expected to be an important fate process (SRC) given a Henry's Law constant of $1.7 X_{10}^{-2}$ atm-cu m/mole (De Bruyn et al 1995). Phosgene is expected to hydrolyze in moist soil; hydrolysis of a $1 \%$ solution of phosgene in water is complete within 20 seconds at o deg C (Mitretek Systems 2000). Phosgene is expected to volatilize from dry soil surfaces (SRC) based upon a vapor pressure of $1420 \mathrm{~mm} \mathrm{Hg}$ (Daubert qand Danner 1989). Biodegradation data were not available (SRC, 2007).

Biota: Phosgene does not accumulate in the food chain. 


\section{Environmental Partitioning}

When released to air, phosgene will exist solely as a gas. Phosgene gas is degraded in the atmosphere by reacting with substances commonly found in the air, but this is a very slow process. Phosgene in the air may also react with moisture in clouds or rain and be broken down into other compounds.

Phosgene will react with water and be broken down into other products. Some of the phosgene that is not broken down may evaporate into air.

When released to soil, phosgene will not stick to the soil. Small amounts may evaporate into air or pass through the soil surface and contaminate groundwater. Most of the phosgene in soil will be broken down when it comes into contact with moisture. 


\section{Regulations}

MCL (Drinking Water): none ug/L

Universal Treatment Standards (UTS): none in wastewater standards and none in non wastewater standards

Groundwater Water Quality Criterion : none ug/L

PQL: none $\mu \mathrm{g} / \mathrm{g}$

IDLH: 2 ppm

EPA RfD: none

EPA RfC: none

\section{Remediation}

Liquid spills can be covered with sodium hydrogen carbonate or an equal mixture of soda ash and slake lime or crystallized urea. Water from an atomizer can then be added cautiously and the mixture transferred to a large volume of water. Gas spills can be mitigated by gaseous ammonia, aqueous ammonia or an ammonia steam curtain or sprays. There should be a holding area beneath any storage or handling installation that can contain a liquid spill. This should have an impermeable flexible membrane liner and must already contain lime, limestone, sodium hydrogen carbonate, urea or any other suitable neutralizing absorbent, sufficient to eliminate the spill (IPCS 2007).

\section{References}

ATSDR_ToxFAQs for Phosgene. http://www.atsdr.cdc.gov/tfacts176.html\#bookmarko3

Atkinson R; (1994) Gas Phase Tropospheric Chemistry of Organic Compounds J Phys Chem Ref Data Monograph No. 2.

Bidleman T.F., (1988)'Atmospheric Processes: Wet and Dry Deposition of Organic Compounds are Controlled by their Vapor-Particle Partitioning. Environmental Science and Technology, 22, 361-367. 
Butler R, Solomon IJ, Snelson A. (1978). Rate constants for the reaction of $\mathrm{OH}$ with halocarbons in the presence of $02+\mathrm{N}_{2}$. J Air Pollut Cont Fed 28:1131-1133.

Daubert TE, Danner RP; (1989) Physical and Thermodynamic Properties of Pure Chemicals Data Compilation. Washington, DC: Taylor and Francis.

De Bruyn, W. J., E. Swartz, J. H. Hu, J. A., Shorter, P. Davidovits, D.R. Worsnop, M. S. Zahniser, and C. E. Kolb, (1995) Henry's Law solubilities and Setchenow coefficients for biogenic reduced sulfur species obtained from gas-liquid uptake measurements, J. Geophys. Res., 100, 7245-7251.

IPCS International Programme on Chemical Safety; Health and Safety Guide No. 106 Phosgene. (1998). Available from, as of July 17, 2007: http://www.inchem.org/documents/hsg/hsg/hsg106.htm.

Kolb, CE, JC Wormhoudt, and MS Zahniser. (1995). Halo Replacements: Technology and Science. Amer Chem Soc, Washington, DC. Miiziolek AW, Tsang W, eds, ACS Symp Ser pp.50-58.

Lyman, WJ, WF Reehl, and DH Rosenblatt (1990) Handbook of Chemical Property Estimation Methods. Washington, DC: American Chemical Society pp. 4-5, 15-1 to 15-29.

Meylan, W., Howard, P.H. and Boethling, R.S. (1992). Molecular topology/fragment contribution method for predicting soil sorption coefficients. Environ. Sci. Technol. 26: 1560-6. (PCKOCWIN Software available from Syracuse Research Corp, Environmental Science Center, Syracuse, NY 13210).

Mitretek Systems; Chemistry of Other Lethal Chemical Agents Database on Phosgene. Available from the Database Query page at http://www.mitretek.org/mission/envene/chemical/agents/other.html as of August 2, 2000.

Montgomery CW, Rollefson GK; (1933) Gas Phase 0xidation of Perhalocarbons. J Amer Chem Soc 55: 4025-35.

SCR. (2007). ChemFate Database. Syracuse Research Corporation, Syracuse, NY.

Swann RL, Laskowski DA, McCall PJ, et al. (1983). A rapid method for the estimation of the environmental parameters octanol/water partition coefficient, soil sorption constant, water to air ratio and water solubility. Res Rev 85:17-28. [(1) Swann RL et al; Res Rev 85: 17-28. 
Talmage, Sylvia S..Nancy B. Munro, Annetta P. Watson, Joseph F. King and Veronique D. Hauschild. (2007) Chemical Warfare Agents: Toxicology and Treatment, Second Edition. John Wiley and Sons, Ltd. 


\section{Phosphamidon 13171-21-6}

\section{Dimethoate 60-51-5 (synonym)}

\section{ENVIRONMENTAL TRANSPORT, DISTRIBUTION, AND TRANSFORMATION}

Air: According to a model of gas/particle partitioning of semivolatile organic compounds in the atmosphere (Bidleman 1988), phosphamidon, which has a vapor pressure of $1.65 \mathrm{X}_{10}^{-5} \mathrm{~mm} \mathrm{Hg}$ at $25 \mathrm{deg} \mathrm{C}$ (Tomlin 1997), will exist in both the vapor and particulate phases in the ambient atmosphere. Vapor-phase phosphamidon is degraded in the atmosphere by reaction with photochemically-produced hydroxyl radicals (SRC); the half-life for this reaction in air is estimated to be $10.5 \mathrm{hrs}$ (SRC), calculated from its rate

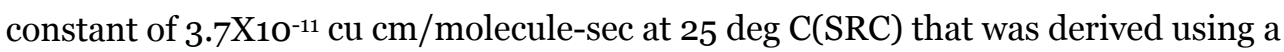
structure estimation method (Meylan and Howard 1993). Particulate-phase phosphamidon may be removed from the air by wet and dry deposition (SRC). The rate constant for the vapor-phase reaction of phosphamidon with ozone has been estimated as $1.6 \mathrm{X}_{10} \mathrm{O}^{-16} \mathrm{cu} \mathrm{cm} / \mathrm{molecule}-\mathrm{sec}$ at $25 \mathrm{deg} \mathrm{C}$ (SRC), derived using a structure estimation method (Bidleman 1988); this corresponds to an atmospheric half-life of about 7 days at an atmospheric concentration of $7 \mathrm{X}_{10}{ }^{11}$ ozone molecules per $\mathrm{cu} \mathrm{cm}$ (Atkinson and Carter 1984).

Water: Based on soil thin layer chromatography study, retardation factors (Rf) of 0.910.92 in a silt loam and a fine sandy loam (Khan and Khan 1986) indicate that phosphamidon is not expected to adsorb to suspended solids and sediment(SRC). Volatilization from water surfaces is not expected (Lyman et al. 1990) based upon an estimated Henry's Law constant of $1.5 \mathrm{X}^{-12} \mathrm{O}^{-12}$ atm-cu m/mole (SRC), developed using a fragment constant estimation method (Meylan and Howard 1991). According to a classification scheme (Franke et al 1994), a BCF of <1(Min and Cha 2000) suggests the potential for bioconcentration in aquatic organisms is low.

Soil: Based on soil thin layer chromatography study, retardation factors (Rf) of 0.91-0.92 in a silt loam and a fine sandy loam(Khan and Khan 1986), indicate that phosphamidon has very high mobility in soil(SRC). Volatilization of phosphamidon from moist soil surfaces is not expected to be an important fate process (Lyman et al. 1990) given an estimated Henry's Law constant of $1.5 \mathrm{X}^{-12} \mathrm{O}^{-12} \mathrm{~atm}-\mathrm{cu} \mathrm{m} / \mathrm{mole}(\mathrm{SRC})$, using a fragment constant estimation method (Meylan and Howard 1991). Phosphamidon is not expected to volatilize from dry soil surfaces (SRC) based upon a vapor pressure of $1.65 \times 10^{-5} \mathrm{~mm}$ $\mathrm{Hg}$ (Tomlin 1997). Half-lives in loam, loamy sand, and sand of approximately 6, 3, and <3 days, respectively (Benyon et al. 1973), indicate that biodegradation of phosphamidon 
may be an important environmental fate process in soil.

Sediment: Based on soil thin layer chromatography study, retardation factors (Rf) of 0.91-0.92 in a silt loam and a fine sandy loam (Khan and Khan 1986) indicate that phosphamidon is not expected to adsorb to suspended solids and sediment (SRC). Volatilization from water surfaces is not expected (Lyman et al. 1990) based upon an estimated Henry's Law constant of $1.5 \mathrm{X}^{-10^{-12}}$ atm-cu m/mole(SRC), developed using a fragment constant estimation method (Meylan and Howard 1991). According to a classification scheme (Franke et al. 1994), a BCF of $<1$ (Min and Cha 2000) suggests the potential for bioconcentration in aquatic organisms is low (SRC). Phosphamidon's hydrolysis half-lives at $23 \mathrm{deg} \mathrm{C}$ are 74 days, 13.8 days, and 2.2 days at $\mathrm{pH}$ 4, 7, and 10, respectively.

\section{Regulatory}

Universal Treatment Standards (UTS): None

Acceptable Daily Intake (ADI) for dimethoate for a human is $0.02 \mathrm{mg} / \mathrm{kg} / \mathrm{day}$

CDHS’ Archived Advisory Levels for Drinking Water: $0.001 \mathrm{mg} / \mathrm{L}$

EPA Rf D: $0.0002 \mathrm{mg} / \mathrm{kg}$-day

EPA RfC: none $\mathrm{mg} / \mathrm{m}^{3}$

PRG: residential soil $\mathrm{mg} / \mathrm{kg}$ industrial soil $\mathrm{mg} / \mathrm{kg}$ ambient air $\mathrm{ug} / \mathrm{m3}$ tap water $\mathrm{ug} / \mathrm{L}$ 120 180

0.73

7.3

\section{Remediation}

Air spill: Apply water spray or mist to knock down vapors. /Organophosphorus pesticides, liquid, flammable, toxic; Organophosphorus pesticides, liquid, toxic; Organophosphorus pesticides, solid, toxic.

Water spill: Use natural barriers or oil spill control booms to limit spill travel. Remove trapped material with suction hoses. /Organophosphorus pesticides, liquid, flammable, toxic; Organophosphorus pesticides, liquid, toxic; Organophosphorus pesticides, solid, toxic.

Land spill: Dig a pit, pond, lagoon, holding area to contain liquid or solid material. /SRP: If time permits, pits, ponds, lagoons, soak holes, or holding areas should be sealed with an impermeable flexible membrane liner. Dike surface flow using soil, sand bags, foamed 
polyurethane, or foamed concrete. Absorb bulk liquid with fly ash, cement powder, or commercial sorbents. Organophosphorus pesticides, liquid, flammable, toxic; Organophosphorus pesticides, liquid, toxic; Organophosphorus pesticides, solid, toxic (Association of American Railroads).

\section{References}

Association of American Railroads/Bureau of Explosives (2002) Emergency Handling of Hazardous Materials in Surface Transportation. Association of American Railroads. Pueblo, CO,, p. 698.

Atkinson, R. and W.P.L. Carter (1984) Kinetics and mechanisms of the gas-phase reactions ... organic compounds under atmospheric conditions, Chem Rev 84: 437-70.

Beynon, K.I, Hutson D.H., and Wright, A.N. (1973) The metabolism and degradation of vinyl phosphate insecticides. Residue Rev 47:55-142.5.

Bidleman, T.F. (1988)“Atmospheric processes: Great Lakes Pesticide Report“Environmental Science and Technology. 22(4):361-367.

Franke, C., G. Studinger, G. Berger, S. Böhling, U. Bruckmann, D. Cohors-Fressenborg and U. Jöhncke (1994), The assessment of bioaccumulation. Chemosphere 29:1501-1514.

Khan S and Khan NN (1986) The Mobility of Some Organophosphorus Pesticides in Soils As Affected By Some Soil Parameters. Soil Sci 142: 214-22.

Lyman, W.J., W.F. Reehl, and D.H. Rosenblatt (1990) Handbook of Chemical Property Estimation Methods. Washington, DC: American Chemical Society pp. 4-9, 15.1-15.29.

Meylan, W.M. and Howard, P.H. (1991) Bond contribution method for estimating Henry's Law Constants. Environ. Toxicol. Chem. 10: 1283-93.

Meylan, W. M. and P. H. Howard (1993) Reliability: Estimate based on known qualitative structure-activity relationships. Chemosphere, 26:2293-99.

Min, K.J. and Cha, C.G. (2000) Determination of the bioconcentration of phosphamidon and profenofos in zebrafish Bull Environ Contam Toxicol 65: 611-17.

Tomlin, C. (1997) The Pesticide Manual World Compendium 11th ed., Surrey, England: British Crop Protection Council p. 965. 


\section{Phosphorous Trichloride $\quad 7719-12-2$}

\section{ENVIRONMENTAL TRANSPORT, DISTRIBUTION, AND TRANSFORMATION}

Air: White phosphorus can enter air from its production, use, accidental spills during loading and unloading for shipment and accidental spills during transport. During white phosphorus production, an estimated $0.58 \mathrm{~kg}$ total phosphorus/ton of product is emitted into the air (EPA 1989). Part of the air emissions during production of white phosphorus from phosphate rock is expected to be in the form of phosphate.

The air emission of elemental phosphorus during production of munitions at the Pine Bluff Arsenal in Arkansas was estimated at $1 \mathrm{mg} / \mathrm{kg}$ of phosphorus used (Berkowitz et al. 1981). The amount of elemental phosphorus released to air during the manufacture of phosphoric acid, phosphates, and other phosphorus chemicals is not known because the air emission factor is estimated for total phosphorus (EPA 1989).

However, the emission of elemental phosphorus from the manufacture of phosphorus chemicals may be low, because the conversion rate from elemental phosphorus to compounds is high. White phosphorus is also released in air during its use as an incendiary device by the military. It will enter the atmosphere during testing, which produces phosphorus smoke in the field. At a smoke density of $0.1 \mathrm{mg} / \mathrm{m}^{3}$, the estimated concentration of elemental phosphorus in the smoke was 21 ppb (EPA 1991).

Water: The very small amounts of phosphorus trichlorides and oxychlorides that actually enter the environment react rapidly with water to form hydrochloric and phosphorus oxyacids. After deposition, these oxyacids are neutralized and/or buffered to form chloride (by oxidation of phosphite) and phosphate salts. The quantities of these chlorides and phosphate salts are not significant.

Sediment and Soil: The exposure of soil to phosphorus aerosols will upset the $\mathrm{pH}$ of the soil and create a more acidic layer of soil. This decrease in $\mathrm{pH}$ can exceed the buffering capacity of the surface layer of the soil depending upon the amount of applied phosphorus. This process can be mitigated by a larger soil volume area so that phosphorus speciation on a field scale will be minimal. The interaction of metals with phosphorus condensates could lead to their leachability and possible trace metal migration from the soil (Van Vorris et al. 1987).

Biota: Phosphorus trichloride and phosphorus oxychloride react with the water component of the tissues they first contact. The resulting hydrogen chloride and 
phosphorus oxyacid ions, if absorbed, join the body pools of these ions. Phosphate, chloride, and hydrogen ions are easily excreted by the kidneys by normal physiological mechanisms.

Phosphorus trichloride and phosphorus oxychloride hydrolyse by reacting 3 moles of water per mole and yielding 6 moles of hydrogen ion per mole. As liquids or in high vapor concentrations, they kill most animal and plant tissues they contact. However, the dehydrating and acidifying effects of major releases are transient and local.

\section{Environmental Partitioning}

The persistence of white phosphorus in air is very short and may range from minutes to days. Particulate white phosphorus present as an aerosol may be coated with a protective layer of oxide and may have a longer lifetime in air (Berkowitz et al. 1981). In addition to aerosol age, phosphorus aerosol speciation is also affected by the humidity of the ambient environment (Van Voris et al. 1987). Washout and rainout processes transport both the reaction products of vapor phase phosphorus and unreacted particles of phosphorus to water and land (Berkowitz et al. 1981). Because of its lower water solubility, physical state (gas), and slower reactivity, phosphine formed during the combustion of white phosphorus or released to the atmosphere from other media persists in the atmosphere longer than other reaction products.

\section{Regulatory}

\section{IDLH: $25 \mathrm{ppm}$}

Universal Treatment Standards (UTS): None

EPA RfD: $10 \mathrm{mg} / \mathrm{kg}$-day

EPA RfC: none $\mathrm{mg} / \mathrm{m}^{3}$

Drinking Water Remedial Target: none $\mu \mathrm{g} / \mathrm{L}$

\section{Remediation}

Water Spill: Neutralize with agricultural lime $(\mathrm{CaO})$, crushed limestone $\left(\mathrm{CaCO}_{3}\right)$, or sodium bicarbonate $\left(\mathrm{NaCO}_{3}\right)$. Use mechanical dredges or lifts to remove immobilized masses of pollutant and precipitates. Adjust $\mathrm{pH}$ to neutral ( $\mathrm{pH}=7)$ Air Spill: Apply water spray or mist to knock down vapors. Vapor knockdown water is corrosive or toxic and should be diked for containment. 
Land Spill: Dike surface flow using soil, sand bags, foamed polyurethane, or foamed concrete. Absorb bulk liquid with fly ash or cement powder. Neutralize with agricultural lime $(\mathrm{CaO})$, crushed limestone $\left(\mathrm{CaCO}_{3}\right)$, or sodium bicarbonate $\left(\mathrm{NaHCO}_{3}\right)$

(Association of American Railroads).

\section{References}

Association of American Railroads; Bureau of Explosives. (2005) Emergency Handling of Hazardous Materials in Surface Transportation. Association of American Railroads, Pueblo, CO. p. 723.

Berkowitz JB, Young GS, Anderson RC, et al. (1981) Research and development for health and environmental hazard assessment, Task Order 5. Occupational and environmental hazards associated with the formulation and use of white phosphorus-felt and red phosphorus-butyl rubber screening smokes. Cambridge, MA: Arthur D. Little, Inc. DAMD17-79-C-9139. AD-Al 16956.

EPA. (1991) White phosphorus: Health advisory. Washington, DC: Office of Drinking Water, U.S. Environmental Protection Agency. EPA 500/8-891072. NTIS PB91-161026/AS.

IPCS, (1997) CEC; International Chemical Safety Card on Phosphorus trichloride. Available from, as of November 03, 2006: http://www.inchem.org/documents/icsc/icsc/eics0696.htm.

Van Voris P, Cataldo DA, Ligotke MW, et al. (1987) Evaluate and characterize mechanisms controlling transport, fate and effects of Army smokes in the aersol wind tunnel. Pacific Northwest Laboratories, Richland, Washington. 


\title{
Phosphoryl trichloride $\quad$ 10025-87-3
}

\section{(Phosphorus oxychloride)}

\section{ENVIRONMENTAL TRANSPORT, DISTRIBUTION, AND TRANSFORMATION}

\begin{abstract}
Air: According to a model of gas/particle partitioning of semivolatile organic compounds in the atmosphere (Bidleman 1988), phosphorus oxychloride, which has a vapor pressure of $40 \mathrm{~mm} \mathrm{Hg}$ at $27.3 \mathrm{deg} \mathrm{C}$ (Lewis 1999), is expected to exist solely as a vapor in the ambient atmosphere. Vapor-phase phosphorus oxychloride is degraded in the atmosphere by reaction with photochemically-produced hydroxyl radicals (SRC); the half-life for this reaction in air is reported to be $>290$ days, calculated from its rate constant of $<4 \mathrm{X}_{10} \mathrm{O}^{-14} \mathrm{cu} \mathrm{cm} /$ molecule-sec at $25 \mathrm{deg}$ (Martin et al. 2002). Phosphorus oxychloride does not contain chromophores that absorb at wavelengths $>290 \mathrm{~nm}$ (Lyman et al. 1990) and therefore is not expected to be susceptible to direct photolysis by sunlight (SRC).
\end{abstract}

Water: Phosphorus oxychloride hydrolyzes rapidly in water at room temperature (Lewis 1999).

Soil: Hydrolysis is expected to be the most important fate process for phosphorus oxychloride in the environment (Lewis 1999). Phosphorus oxychloride is expected to volatilize from dry soil surfaces (SRC) based upon a vapor pressure of $40 \mathrm{~mm} \mathrm{Hg}$ (Lewis 1999).

Biota: Hydrolyzes rapidly in water at room temperature (Lewis 1999), and therefore is not expected to bioconcentrate in organisms.

\section{Regulatory}

MCL (Drinking Water): none

Universal Treatment Standards (UTS): None

EPA RfD: none mg/kg-day

EPA RfC: none $\mathrm{mg} / \mathrm{m}^{3}$

Drinking Water Remedial Target: none $\mu \mathrm{g} / \mathrm{L}$ 


\section{Remediation}

Keep material out of water sources and sewers; build dikes to contain flow as necessary; use water spray to knock down vapors; do not use water on material itself; and neutralize spilled material with crushed limestone, soda ash, or lime. For a land spill, dig a pit, pond, lagoon, or holding area to contain liquid or solid material; dike surface flow using soil, sand bags, foamed polyurethane, or foamed concrete; absorb bulk liquid with fly ash or cement powder; neutralize with agricultural lime (slaked lime), crushed limestone, or sodium bicarbonate. For a water spill, neutralize with agricultural lime (slaked lime), crushed limestone, or sodium bicarbonate; use mechanical dredges or lifts to remove immobilized masses of pollutants and precipitates; adjust $\mathrm{pH}$ to neutral ( $\mathrm{pH}$ 7). For air spills apply water spray or mist to knock down vapors; vapor knock down water is corrosive or toxic and should be diked for containment. Stop leak if you can do so without risk. Do not touch spilled material. Keep combustibles (wood, paper, oil, etc.) away from spilled material. Clean up only under supervision of an expert. Keep this chemical out of a confined space, such as a sewer, because of the possibility of an explosion, unless the sewer is designed to prevent the build-up of explosive concentrations. It may be necessary to contain and dispose of this chemical as a hazardous waste. If material or contaminated runoff enters water ways, notify downstream users of potentially contaminated waters (Sittig 2002).

\section{References}

Bidleman, T.F. (1988) “Atmospheric processes: Great Lakes Pesticide Report"Environmental Science and Technology. 22(4):361-367.

Lewis, R.J. (1999); Sax's Dangerous Properties of Industrial Materials, 10th ed, New York, NY: John Wiley \& Sons Inc 3: 2954

Lyman WJ et al; (1990) Handbook of Chemical Property Estimation Methods. Washington, DC: Amer Chem Soc pp. 8-12

Martin P et al (2002) J Phys Chem 106: 1542-1550 .

Sittig, M. (2002) Handbook of Toxic and Hazardous Chemicals and Carcinogens, 2002. 4th ed. Norwich, NY: Noyes Publications, 1(A-H):1871.

U.S. Department of Health and Human Services. Hazardous Substances Data Bank (HSDB), a database of the National Library of Medicine's TOXNET system. Bethesda, MD. http://toxnet.nlm.nih.gov. 


\section{Potassium Chloride $\quad 7447-40-7$}

\section{ENVIRONMENTAL TRANSPORT, DISTRIBUTION, AND TRANSFORMATION}

Potassium Chloride $(\mathrm{KCl})$ is ubiquitous in the environment, occurring in minerals, soil and sediments, and natural waters (oceans, lakes, rivers). $\mathrm{KCl}$ as inorganic salt is not subjected to further degradation processes in the environment.

Water: In water, potassium chloride is highly water soluble, and readily undergoes dissociation. In soil, transport/leaching of potassium and chloride is affected by clay minerals (type and content), $\mathrm{pH}$, and organic matter (Betlach et al.1987, Biesinger and Christensen 1972). $\mathrm{K}$ is in general less mobile and less prone to leaching than anions in soil, such as chloride and nitrate $\left(\mathrm{NO}_{3}{ }^{-}\right)$. Chloride binds only weakly to soil particles, and therefore follows water movement. Anions do not leach alone, but always together with a counter-ion (cation). Chloride and nitrate leaching may deplete soils of nutrients such as $\mathrm{K}$. Loss of potassium by erosion or leaching carries no environmental concern. In one published study carried out under national guidelines and with reliable procedure description, the following results on acute aquatic toxicity were found: Pimephales promelas: 96 h-LC50 = 880 mg/l ( $\pm \square 15)$; Daphnia magna: 48h EC50 = 177 mg/l; Ceriodaphnia dubia: $48 \mathrm{~h}-\mathrm{EC} 50=630 \mathrm{mg} / \mathrm{l}( \pm 14)$ (Lewis 1999). For fish species, these studies show effects in the similar range of doses: two studies on Lepomis macrochirus (Lyman 1983, 1983), one study on Oncorhyncus mykiss and one study on Ictalurus punctatus showed $48 \mathrm{~h}-\mathrm{LC} 50$ values in the range 720-2010 mg/l (Marschner1995). The toxicity of $\mathrm{KCl}$ has been investigated in one algae species (Nitzschia linearis), showing 120 h-EC50 (growth rate) of $1337 \mathrm{mg} / \mathrm{l}$ (Lyman 1983).

Soil: Agricultural soil productivity is dependent on establishing a balanced availability of potassium throughout the growing season, often resulting in the need of fertilizing with potassium. Inadequate maintenance of $\mathrm{K}$ in agricultural soils will lead to loss of soil productivity. K deficiencies may also restrict a crop's ability to utilize $\mathrm{N}$, resulting in increased potential for nitrate leaching, and lack of K can enhance a crop's susceptibility to disease and increase the need for pesticide application.

Plants: Toxicity to terrestrial plants: Potassium is one of the three major nutrients and chloride is an essential micronutrient for plants. Literature search has not revealed studies related to toxic effects on terrestrial organisms, therefore this section only describes levels of potassium necessary for good growth of plants and its role in plant physiology (Betlach et al.1987, Biesinger and Christensen 1972, Mitchell et al. 1988). 
Demands for potassium and chloride in plants/crops: The potassium requirement for optimal plant growth is in the range 2-5\% of the plant dry weight of vegetative parts. In crops, uptake of $\mathrm{K}$ in plants ranges from about 50 to $300 \mathrm{~kg} \mathrm{~K}$ ha- 1 per crop, broadly similar to the uptake of nitrogen. Inadequate supply of potassium makes plants more susceptible to frost damage, fungal attack and drought. In most plant species the $\mathrm{Cl}$ requirement for optimal growth is in the range of 0.2-0.4 mg/g dry matter. Excessive $\mathrm{Cl}$ is detrimental to chloride-sensitive crops, or if it is necessary to guard against excessive salinity, and in arid areas $\mathrm{Cl}$ deficiency may occur in highly leached soils with a low $\mathrm{Cl}$ input from rain. The principal effect of $\mathrm{Cl}$ deficiency in plants is a reduction in leaf surface area and thereby plants dry weight. With severe deficiency, necrosis might occur.

Environmental Partitioning: Environmental distribution of potassium chloride using a Generic Level I Fugacity Model under three emission scenarios showed that $\mathrm{KCl}$ is mainly distributed to water ( $99.7 \%$ ), while other compartments make minor contributions (< $0.03 \%$ to air, $<0.03 \%$ to soil solids, $<0.01 \%$ to sediment solids). This result applied under the three emissions scenarios $100 \%$ release to water, $100 \%$ release to air, or $100 \%$ release to soil [12]. Fugacity estimations may not be reliable for $\mathrm{KCl}$. The modelling programs used are designed for organic chemicals and the chemical is not expected to undergo photolysis or biodegradation. Taking into consideration the physico-chemical properties of potassium chloride, and a calculated octanol/water partition coefficient of 0.46 , no potential for bioaccumulation/ bioconcentration can be identified.

\section{Regulatory}

MCL (Drinking Water): none

Universal Treatment Standards (UTS: none in wastewater standards

NIOSH: $=1500 \mathrm{mg} / \mathrm{kg}$ Oral LD50 Mouse $=\mathbf{2 6 0 0} \mathrm{mg} / \mathrm{kg}$ Oral LD50 Rat

EPA RfD: none

EPA RfC: none

\section{Remediation}

$\mathrm{KCl}$ does not pose a threat. 
Spills/Leaks: Clean up spills immediately, observing precautions in the Protective Equipment section. Sweep up or absorb material, then place into a suitable clean, dry, closed container for disposal. Avoid generating dusty conditions. Provide ventilation.

\section{References}

Betlach, C.J., Arnold, J.D, Frost, R.W., Leese, P.T., Gonzalez, M.A. (1987) Bioavailability and pharmokonetics of a new sustained-release potassium chloride tablet. Parm. Res. 4(5):409-411.

Biesinger, K., Christensen, G.L. (1972) Effects of various metals on survival, growth, reproduction and metabolism of Daphnia magna. J. Fish. Res. Bd. Canada 29:1691-1700.

Lyman, W.J., Reehl, W.J., Rosenblatt, D.H. (1983) Handbook of chemical property estimation methods. McGraw-Hill, New York. p. 5-10.

Lyman, W.J., Reehl, W.J., Rosenblatt, D.H. (1983) Handbook of chemical property estimation methods. McGraw-Hill, New York. p. 5-4.

Marschner, H. (1995). Mineral Nutrition of higher Plants. 2nd ed Academic Press, London.

Mitchell, A.D., Rudd, C.J., Caspary, W.J. (1988) Evaluation of the L5178Y mouse lymphoma cell mutagenesis assay: Intralaboratory results for sixty-three coded chemicals tested at SRI international. Env. Molecular Mutagenesis 12(13):37-101. 


\section{Potassium Cyanide $\quad$ 151-50-8}

\section{ENVIRONMENTAL TRANSPORT, DISTRIBUTION, AND TRANSFORMATION}

Air: Most cyanide in the atmosphere exists almost entirely as hydrogen cyanide gas, although small amounts of metal cyanides may be present as particulate matter in the air (EPA 1984). Hydrogen cyanide is very resistant to photolysis at wavelengths of normal sunlight (EPA 1979). The most important reaction of hydrogen cyanide in air is the reaction with photochemicallygenerated hydroxyl radicals and subsequent rapid oxidation to carbon monoxide (CO) and nitric oxide (NO); photolysis and reaction with ozone are not important transformation processes, and reaction with singlet oxygen (O 1D) is not a significant transformation process except at stratospheric altitudes where singlet oxygen is present in significant concentrations (Cicerone and Zellner 1983). The rate of hydroxyl radical reaction with hydrogen cyanide in the atmosphere depends on the altitude, and the rate of the reaction is at least an order of magnitude faster at lower tropospheric altitudes $(0-8 \mathrm{~km})$ than at upper tropospheric altitudes $(10-12 \mathrm{~km})$ (Cicerone and Zellner 1983). Based on a reaction rate constant of 3x10-14 cm3/(molecule-sec) at $25{ }^{\circ} \mathrm{C}$ (Fritz et al. 1982) and assuming an average hydroxyl radical concentration of $5 \times 105$ molecules $/ \mathrm{cm} 3$, the residence time for the reaction of hydrogen cyanide vapor with hydroxyl radicals in the atmosphere is $\approx 2$ years. This value compares well with the atmospheric residence time derived by Cicerone and Zellner (1983) of approximately 2.5 years, with a range of 1.3-5.0 years, depending on the hydroxyl radical concentrations assumed. Using the equation $t^{1} / 2=0.693 \tau$ for converting residence time $(\tau)$ to half-life ( $\left.t^{1} / 2\right)$ (Lyman 1982) and an estimated atmospheric residence time for hydrogen cyanide of 2-3 years, and assuming first-order kinetics for the reaction of hydrogen cyanide with hydroxyl radicals, an atmospheric half-life of 1.4- 2.9 years can be calculated for hydrogen cyanide.

Water: Cyanide occurs most commonly as hydrogen cyanide in water, although it can also occur as the cyanide ion, alkali and alkaline earth metal cyanides (potassium cyanide, sodium cyanide, calcium cyanide), relatively stable metallocyanide complexes (ferricyanide complex $\left[\mathrm{Fe}(\mathrm{CN})_{6}\right]^{-3}$ ), moderately stable metallocyanide complexes (complex nickel and copper cyanide), or easily decomposable metallocyanide complexes (zinc cyanide $[\mathrm{Zn}(\mathrm{CN}) 2]$, cadmium cyanide $\left[\mathrm{Cd}(\mathrm{CN})_{2}\right]$ ). The environmental fate of these cyanide compounds varies widely (EPA 1979).

Biota: Most of the available information on the mechanisms of biodegradation of cyanides in water comes from studies on the evaluation and use of this process as a means of detoxifying cyanide-containing wastes (Akcil and Mudder 2003; EPA 1994; Raybuck 1992). It is known that there is a natural attentuation of the cyanide ion and thiocyanide concentrations in waste waters, for example reduction in gold mill tails that is due the acclimation of indigenous microflora in the tailings (Akcil and Mudder 2003; Oudjehani et al. 2002; Zagury et al. 2004). A number of microorganisms have been identified that are capable of uptake, conversion, sorption, and/or precipitation of the cyanide ion, cyanate, and thiocyanate, including species of the 
genera, Actinomyces, Alcaligenes, Arthrobacter, Bacillus, Micrococcus, Neisseria, Paracoccus, Pseudomonas, and Thiobacillus (Akcil and Mudder 2003). Some of these species, for example Pseudomonas, are capable of using the cyanide ion and thiocyanate as the sole source of carbon and nitrogen and therefore, are particularly effective at cyanide degradation. In fact, Pseudomonas is the basis of commercial applications for degrading the cyanide ion to ammonia and carbonate in waste waters generated in mining operations that use the cyanide ion to leach gold and other precious metals for low-grade ores (Akcil and Mudder 2003).

Sediment and Soil: Analogous to the fate of cyanides in water, it is predicted that the fate of cyanides in soil would be dependent on cyanide concentrations, $\mathrm{pH}$, temperature, metal content, concentration of microbes, availability of nutrients, and acclimation of microbes. Cyanide may occur as hydrogen cyanide, alkali metal salts, or as immobile metallocyanide complexes. In soil, cyanide present at low concentrations would biodegrade under aerobic conditions with the initial formation of ammonia, which would be converted to nitrite and nitrate in the presence of nitrifying bacteria. Under anaerobic conditions, the cyanides ion will denitrify to gaseous nitrogen (Richards and Shieh 1989). Upper limits of 200 and $2 \mathrm{ppm}\left(\mathrm{mg} / \mathrm{kg} \mathrm{CN}^{-}\right)$, respectively, have been reported for uninhibited aerobic and anaerobic biodegradation of cyanide in soil (Fueller 1985); however, these limits have not been confirmed in other studies (Thomas and Lester 1993). Cyanide ions in soil are not involved in oxidation-reduction reactions but may undergo complexation reactions with metal ions in soil (EPA 1978).

Environmental partitioning: Because hydrogen cyanide is a gas and has a relatively slow degradation rate in air, the atmosphere will be the ultimate sink for this compound. Almost all of the hydrogen cyanide released to the atmosphere remains in the lower altitudes (troposphere); only $2 \%$ of tropospheric hydrogen cyanide is transferred to the stratosphere (Cicerone and Zellner 1983). Cyanide has the potential to be transported over long distances from its emission source. Despite higher water solubility at saturated pressure, the removal of hydrogen cyanide by rainwater appears to be a negligible partitioning pathway (Cicerone and Zellner 1983). Because hydrogen cyanide is a gas, its removal from air by dry deposition is also likely to be negligible. However, metal cyanide particles, particularly water-soluble cyanide particles, are expected to be removed from the air by both wet and dry deposition.

Volatilization and sorption are the two physical processes that contribute to the loss of cyanide from water. At $\mathrm{pH}<9.2$, most of the free cyanide in solution should exist as hydrogen cyanide, a volatile cyanide form (EPA 1978). On the basis of Henry's law constant and the volatility characteristics associated with various ranges of Henry's law constant (Thomas 1982), volatilization is a significant and probably dominant fate process for hydrogen cyanide in surface water (EPA 1992). The most common alkali metal cyanides (e.g., sodium and potassium cyanide) may also be lost from surface water primarily through volatilization; whereas, the sparingly soluble metal cyanides such as copper (I) cyanide are removed from water predominantly by sedimentation and biodegradation (EPA 1992). Variations in the volatilization rate are expected because this process is affected by several parameters including temperature, $\mathrm{pH}$, wind speed, and cyanide concentration (EPA 1979). The volatilization rate was $\mathrm{pH}$-dependent, with the rate faster at a lower $\mathrm{pH}$. Data indicated that cyanide volatilization is a more important fate process than cyanide loss due to chemical and biodegradation reactions (Ludzack et al. 1951; Raef et al. 1977). Because volatilization is not an important fate process for cyanide in groundwater, cyanide would be expected to persist for considerably longer periods of time in underground aquifers than in surface water. 


\section{Regulatory}

MCL (Drinking Water): $0.2 \mathrm{mg} / \mathrm{L}$ cyanide

Universal Treatment Standards (UTS): $1.2 \mathrm{mg} / \mathrm{l}^{2}$ in wastewater standard

$590 \mathrm{mg} / \mathrm{L}$ in nonwastewater standard

EPA RfD: $0.05 \mathrm{mg} / \mathrm{kg}$-day

EPA RfC: none $\mathrm{mg} / \mathrm{m}^{3}$

Soil PQL: $3 \mathrm{mg} / \mathrm{kg}$

PRG: residential soil mg/kg industrial soil mg/kg ambient air ug/m^3 tap water $\mathrm{ug} / \mathrm{I}$

$\begin{array}{llll}11 & 35 & 3.1 & 6.2\end{array}$

\section{Remediation}

Biodegradation of cyanides has been investigated, with varying results, for several industrial processes, and additional research in this area would be valuable. While investigations of the potential for microbial species found in mineral processing waste waters demonstrate effective removal of cyanide, metal complexed cyanide, and thiocyanate (Boucabeille 1994; EPA 1994), complex cyanides did not appear amenable to biodegradation at gasworks sites (Thomas and Lester 1993). Application of formaldehyde to electroplating waste under basic conditions can convert the cyanide anion to substituted acetates in addition to recovering copper and silver as free metals with formaldehyde reduction (Tucker and Carson 1985). Calcium or sodium polysulfide treatment converts some cyanide wastes into less toxic thiocyanate (Higgins and Desher 1988). These examples suggest that typical treatments involve the decomposition of cyanides to less toxic compounds by physical or chemical processes. More than $97 \%$ of cyanide is typically removed from waste waters by alkaline chlorination, electrolysis, or ozonation process. Cyanide from some wastes can be removed by ion-exchange resins. After using an appropriate treatment method such as those described above, cyanide wastes may be disposed of in a secured sanitary landfill (Grosse 1986; Higgins and Desher 1988; Tucker and Carson 1985). Disposal by injection of high-pH cyanide wastes into sandstone was investigated by Scrivner et al. (1986). Currently, the injection of waste water containing hydrogen cyanide and cyanide compounds through underground injection is a major method for disposal of these wastes. 


\section{References}

Akcil A, Mudder T. (2003) Microbial destruction of cyanide wastes in gold mining:

Process review. Biotechnol Lett 25:445-450.

Boucabeille C, Bories A, Ollivier P, et al. (1994) Microbial degradation of metal complexed cyanides and thiocyanate from mining wastewaters. Environ Poll 84(1):59-67.

Cicerone RJ, Zellner R. (1983) The atmospheric chemistry of hydrogen cyanide (HCN). J Geophys Res 88:10689-10696.

EPA. (1978) Reviews of the environmental effects of pollutants. V. Cyanide. Cincinnati, OH: U.S. Environmental Protection Agency Health Effects Research Laboratory, Office of Research and Development. PB289920.

EPA. (1979) Cyanides. In: Water-related environmental fate of 129 priority pollutants. Vol. 1. Washington, DC: U.S. Environmental Protection Agency, Office of Water Planning and Standards, Office of Water and Waste Management. EPA440479029a. PB80204373. 12.1-12.12.

EPA. (1984) Health effects assessment for cyanide. Washington, DC: U.S. Environmental Protection Agency. EPA540186011.

EPA. (1992) U.S. Environmental Protection Agency. Fed Regist 57:26248.

EPA. (1994) Technical report: Treatment of cyanide heap leaches and tailings.

Washington, DC: U.S. Environmental Protection Agency, Office of Solid Waste. EPA530R94037. PB94201837.

Fueller WH. (1985) Cyanides in the environment with particular attention to the soil. In: Van Zyl D, ed. Cyanide and the environment: Proceedings of a conference Tucson, Arizona December 11-14, 1984, Fort Collins, CO: Colorado State University, 1:19-46.

Grosse DW. (1986) Treatment technologies for hazardous wastes part IV. A review of alternative treatment processes for metal-bearing hazardous waste streams. J Air Pollut Control Assoc 36:603-614.

Higgins TE, Desher DP. (1988) Metal finishing and processing. J Water Pollut Control Fed 60:904-909. 
Lyman, W. (1982) Atmospheric residence time. In: Lyman WJ, Reehl WF, Rosenblatt DH, eds. Handbook of chemical property estimation methods: Environmental behavior of organic compounds. New York, NY: McGraw Hill Book Company, pp. 10.2-10.33.

Ludzack FJ, Moore WA, Krieger HL, et al. (1951) Effect of cyanide on biochemical oxidation in sewage and polluted water. Sewage Ind Wastes 23:1298-1307.

Oudjehani K, Zagury GJ, Deschênes L. (2002) Natural attenuation potential of cyanide via microbial activity in mine tailings. Appl Microbiol Biotechnol 58:409-415.

Raef SF, Characklis WG, Kessick MA, et al. (1977) Fate of cyanide and related compounds in aerobic microbial systems--II. Microbial degradation. Water Res 11:485-492.

Raybuck SA. (1992) Microbes and microbial enzymes for cyanide degradation. Biodegradation 3(1):3 18.

Richards DJ, Shieh WK. (1989) Anoxic-oxic activated-sludge treatment of cyanides and phenols. Biotechnol Bioeng 33:32-38.

Scrivner NC, Bennett KE, Pease RA, et al. (1986) Chemical fate of injected wastes. Ground Water Monit Rev 6:53-58.

Thomas RG. (1982) Volatilization from water. In: Lyman WJ, Reehl, WF, Rosenblatt DH, eds. Handbook of chemical property estimation methods: Environmental behavior of organic compounds. New York, NY: McGraw-Hill Book Co., pp. 15-16.

Thomas AO, Lester JN. (1993) The microbial remediation of former gasworks sites. A review. Environ Technol 14(1):1-24.

Tucker SP, Carson GA.(1985) Deactivation of hazardous chemical wastes. Environ Sci Technol 19:215-220.

Zagury GJ, Oudjehani K, Deschenes L. (2004) Characterization and availability of cyanide in solid mine tailings from gold extraction plants. Sci Total Environ 320:211-224. 


\title{
Propylene Oxide 75-56-9
}

\section{ENVIRONMENTAL TRANSPORT, DISTRIBUTION, AND TRANSFORMATION}

\begin{abstract}
Air: According to a model of gas/particle partitioning of semivolatile organic compounds in the atmosphere (Bidleman 1988), 1,2-propylene oxide, which has a vapor pressure of $538 \mathrm{~mm} \mathrm{Hg}$ at $25 \mathrm{deg} \mathrm{C}$ (Boublik et al. 1984) is expected to exist solely as a vapor in the ambient atmosphere. Vapor-phase 1,2-propylene oxide is degraded in the atmosphere by reaction with photochemically-produced hydroxyl radicals(SRC); the half-life for this reaction in air is estimated to be 30 days (SRC), calculated from its rate constant of $5.2 \mathrm{X}^{-12} \mathrm{c}^{-12} \mathrm{cu} \mathrm{cm} / \mathrm{molecule}-\mathrm{sec}$ at $25 \mathrm{deg} \mathrm{C}$.
\end{abstract}

Water: Based on a classification scheme (Swann et al. 1983), an estimated Koc value of 25, determined from an measured log Kow of 0.03 and a regression-derived equation (Lyman et al. 1994), indicates that 1,2-propylene oxide is not expected to adsorb to suspended solids and sediment (SRC). Volatilization from water surfaces is expected (Lyman et al. 1994) based upon an estimated Henry's Law constant of 6.96X10-5 atm-cu $\mathrm{m} /$ mole, based upon its vapor pressure, $538 \mathrm{~mm} \mathrm{Hg}$ (Boublik et al. 1984), and water solubility, 5.9X105 mg/l (Bogyo et al. 1980). Using this Henry's Law constant and an estimation method (Lyman et al. 1994), volatilization half-lives for a model river and model lake are 12 hours and 6 days, respectively (SRC). According to a classification scheme (Franke et al. 1994), an estimated BCF of 3 (SRC), from its log Kow (Hansch et al. 1995) and a regression- derived equation (Meylan et al. 1999), suggests the potential for bioconcentration in aquatic organisms is low (SRC). In freshwater, propylene oxide will hydrolyze with estimated half-lives of 11.6 days (pH's 7-9) and 6.6 days ( $\mathrm{pH} 5$ ) at 25 deg C (Bogyo et al. 1980). 1, 2-Propylene oxide, present at $100 \mathrm{mg} / \mathrm{l}$, reached $95 \%$ of its theoretical BOD in 3 weeks using an activated sludge inoculum at $30 \mathrm{mg} / \mathrm{l}$ and the Japanese MITI test (CITI 1992), suggesting biodegradation will be an important fate process.

Soil: An estimated Koc value of 25 (SRC), determined from a measured log Kow of 0.03 (Hansch et al. 1995) and a regression-derived equation (Lyman et al. 1994), indicates that 1, 2-propylene oxide is expected to have very high mobility in soil (Swann et al. 1983). Volatilization of 1,2-propylene oxide from moist soil surfaces is expected to be an important fate process (SRC) given an estimated Henry's Law constant of 6.96X10-5 atmcu m/mole (SRC), calculated from its vapor pressure, $538 \mathrm{~mm} \mathrm{Hg}$ (Boublik et al. 1984), and water solubility, $5.9 \mathrm{X}_{10} \mathrm{mg} / \mathrm{l}$ (Bogyo et al. 1980). The potential for volatilization of 1,2-propylene oxide from dry soil surfaces may exist (SRC) based upon a vapor pressure of $538 \mathrm{~mm} \mathrm{Hg}$ (Boublik et al. 1984). The aqueous hydrolysis of propylene oxide occurs at 
an environmentally important rate; therefore, hydrolysis in moist soil is likely to be important (Bogyo et al. 1980). 1,2-Propylene oxide, present at $100 \mathrm{mg} / \mathrm{l}$, reached 95\% of its theoretical BOD in 3 weeks using an activated sludge inoculum at $30 \mathrm{mg} / \mathrm{l}$ and the Japanese MITI test (CITI 1992), suggesting biodegradation will be an important fate process.

Biota: An estimated BCF of 3 was calculated for 1,2-propylene oxide(SRC), using a log Kow of 0.03 (Hansch et al. 1995) and a regression-derived equation (Meylan et al. 1994). According to a classification scheme (Franke et al. 1994), this BCF suggests the potential for bioconcentration in aquatic organisms is low.

\section{Regulatory}

MCL (Drinking Water): none

Universal Treatment Standards (UTS): None

OPPTS RfD: $0.001 \mathrm{mg} / \mathrm{kg}$-day

EPA RfC: $0.03 \mathrm{mg} / \mathrm{m} 3$

Drinking Water Remedial Target: 35 g/L (see EPA 2005 c)

PRG: residential soil $\mathrm{mg} / \mathrm{kg}$ industrial soil $\mathrm{mg} / \mathrm{kg}$ ambient air $\mathrm{ug} / \mathrm{m3}$ tap water $\mathrm{ug} / \mathrm{L}$
1.9
9.1
0.52
0.22

\section{Remediation}

Land spill: Dig a pit, pond, lagoon, or holding area to contain liquid or solid material. If time permits, pits, ponds, lagoons, soak holes, or holding areas should be sealed with an impermeable flexible membrane liner. Dike surface flow using soil, sand bags, foamed polyurethane, or foamed concrete. Absorb bulk liquid with fly ash, cement powder, sawdust, or commercial sorbents. Apply "universal" gelling agent to immobilize spill. Water spill: Use natural barriers or oil spill control booms to limit spill. Use surface active agent (eg detergent, soaps, alcohols) to compress and thicken spilled material. Inject "universal" gelling agent to solidify encircled spill and increase effectiveness of booms. If dissolved, apply activated carbon at ten times the spilled amount in region of $10 \mathrm{ppm}$ or greater concentration. Use mechanical dredges or lifts to remove immobilized masses of pollutants and precipitates.

Air release: Apply water spray or mist to knock down vapors (Association of American Railroads 1994). 


\section{References}

Association of American Railroads. (1994) Emergency Handling of Hazardous Materials in Surface Transportation. Washington, DC: Association of American Railroads, Bureau of Explosives, p. 925.

Atkinson R; (1989) Kinetics and mechanisms of the gas-phase reactions of the hydroxyl radical with organic compounds. J Phys Chem Ref Data Monograph 1.

Bidleman T. F. (1988) Atmospheric processes. Envir. Sei. Technol. 22:361-367.

Bogyo DA, Lande SS, and Meylan WM (1980) Investigation of Selected Environmental Contaminants: Epoxides. Washington, DC: USEPA, USEPA-560/11-80-005. pp 202.

Boublik, T., Fried, V., and Hala, E. (1984). The Vapor Pressures of Pure Substances: Selected Values of the Temperature Dependence of the Vapor Pressures of Some Pure Substances in the Normal and Low Temperature Region. Amsterdam, Netherlands: Elsevier Sci Publ. 17.

Chemicals Inspection and Testing Institute (CITI) (1992). Japan Chemical Industry Ecology - Toxicology and Information Center. ISBN 4-89074-101-1

EPA (2005) Propylene Oxide - Revised HED Risk Assessment for Reregistration Eligibility Decision (RED) , PC Code: 042501, DP Barcode: D321759, Office of Prevention, Pesticides, and Toxic Substances, Washington, DC (Sept. 26);

http://cfpub.epa.gov/oppref/rereg/ status.cfm?show=rereg.

Franke, C., G. Studinger, G. Berger, S. Böhling, U. Bruckmann, D. Cohors-Fressenborg and U. Jöhncke (1994) The assessment of bioaccumulation. Chemosphere 29: 1501-14

Hansch, C., Leo, A., and Hoekman, D. (1994) Exploring QSAR. Hydrophobic, Electronic, and Steric Constants. ACS Prof Ref Book. Washington, DC: American Chemical Society, p. 3 .

Lyman, WJ, WF Reehl, and DH Rosenblatt (1990) Handbook of Chemical Property Estimation Methods. Washington, DC: American Chemical Society pp. 4.9, 15.1-15.29.

Meylan, WM; Howard, PH; and Boethling, RS (1999) Improved method for estimating bioconcentration/bioaccumulation factor from octanol/water partition coefficient. Environ. Toxicol. Chem. 18: 664-672 . 
Swann, R.L., Laskowski, D.A., McCall, P.J., van der Kuy, K. and Dishburger, H.J. (1983) A rapid method for estimation of the environmental parameters octanol/water partition coefficient, soil sorption constant water to air ratio and water solubility. Residue Rev. 85, pp. 17-28. (http://toxnet.nIm.nih.gov/cgi-bin/sis/search/r?dbs+hsdb:@term+@rn+60-34-4). 


\title{
Sodium Arsenite 7784-46-5
}

\section{ENVIRONMENTAL TRANSPORT, DISTRIBUTION, AND TRANSFORMATION}

\begin{abstract}
Air: Arsenic is released into the atmosphere primarily as arsenic trioxide or, less frequently, in one of several volatile organic compounds, mainly arsines (EPA 1982b). Trivalent arsenic and methyl arsines in the atmosphere undergo oxidation to the pentavalent state (EPA 1984), and arsenic in the atmosphere is usually a mixture of the trivalent and pentavalent forms (EPA 1984; Scudlark and Church 1988). Photolysis is not considered an important fate process for arsenic compounds (EPA 1979).
\end{abstract}

Water: Arsenic in water can undergo a complex series of transformations, including oxidation-reduction reactions, ligand exchange, precipitation, and biotransformation (EPA 1979, 1984; Sanders et al. 1994; Welch et al. 1988). Rate constants for these various reactions are not readily available, but the factors most strongly influencing fate processes in water include $\mathrm{Eh}, \mathrm{pH}$, metal sulfide and sulfide ion concentrations, iron concentrations, temperature, salinity, distribution and composition of the biota, season, and the nature and concentration of natural organic matter (Farago 1997; Redman et al. 2002; Wakao et al. 1988). Organic arsenical pesticides, such as MSMA, DSMA, and DMA do not degrade by hydrolysis or by aquatic photolysis (EPA 2006). No formation of arsine gas from marine environments has been reported (Tamaki and Frankenberger 1992). Inorganic species of arsenic are predominant in the aquatic environment. In the $\mathrm{pH}$ range of natural -waters, the predominant aqueous inorganic $\mathrm{As}(\mathrm{V})$ species are the arsenate ions, $\mathrm{H}_{2} \mathrm{AsO}_{4}$ and $\mathrm{HAsO}_{4}{ }^{2-}$; the predominant inorganic $\mathrm{As}(\mathrm{III})$ species is $\mathrm{As}(\mathrm{OH})_{3}$ (Aurillo et al. 1994; EPA 1982b). As(V) generally dominates in oxidizing environments such as surface water and As(III) dominates under reducing conditions such as may occur in groundwater containing high levels of arsenic. However, the reduction of arsenate to arsenite is slow, so arsenate can be found in reducing environments. Conversely, the oxidation of arsenite in oxidizing environments is moderately slow (half-life, $0.4-7$ days in coastal systems) and therefore, arsenite can be found in oxidizing environments (Mariner et al. 1996; Sanders et al. 1994). The main organic species in fresh water are MMA and DMA; however, these species are usually present at lower concentrations than inorganic arsenic species (Eisler 1994). Aquatic microorganisms may reduce the arsenate to arsenite, as well as methylate arsenate to its mono- or dimethylated forms (Aurillo et al. 1994; Benson 1989; Braman and Foreback 1973; Edmonds and Francesconi 1987; Sanders et al. 1994). Methylated species are also produced by the biogenic reduction of more complex organoarsenic compounds like arsenocholine or arsenobetaine.

Sediment and Soil: In soil, arsenic is found as a complex mixture of mineral phases, such as co-precipitated and sorbed species, as well as dissolved species (Roberts et al. 2007). The degree of arsenic solubility in soil will depend on the amount of arsenic distributed between these different mineral phases. The dissolution of arsenic is also affected by particle size. The distribution between these phases may reflect the arsenic source (e.g., pesticide application, wood treatment, tanning, or mining operations), and may change with weathering and associations with iron and manganese oxides and phosphate minerals in the soil (Roberts et al. 2007; Ruby et al. 1999). Davis et al. (1996) reported that in soil in Anaconda, Montana, a smelting site from 1860 to 1980, contained arsenic that is only in a sparingly soluble form, consisting of primarily arsenic oxides and phosphates. 
The arsenic cycle in soils is complex, with many biotic and abiotic processes controlling its overall fate and environmental impact. Arsenic in soil exists in various oxidation states and chemical species, depending upon soil $\mathrm{pH}$ and oxidation-reduction potential. Under most environmental conditions, inorganic As (V) will exist as a mixture of arsenate anions, $\mathrm{H}_{2} \mathrm{AsO}_{4}$ and $\mathrm{HAsO}_{4}{ }^{2-}$, and inorganic As (III) will exist as $\mathrm{H}_{3} \mathrm{AsO}_{3}$. The arsenate and arsenite oxyanions have various degrees of protonation depending upon $\mathrm{pH}$ (EPA 1982b; McGeehan 1996). As (V) predominates in aerobic soils, and As (III) predominates in slightly reduced soils (e.g., temporarily flooded) or sediments (EPA 1982b; Sanders et al. 1994). As (III) commonly partitions to the aqueous phase in anoxic environments, and would be more mobile. As (V) usually remains bound to minerals, such as ferrihydrite and alumina, limiting its mobility and bioavailability (Rhine et al. 2006).

Arsenite is moderately unstable in the presence of oxygen; however, it can be found under aerobic conditions as well (Sanders et al. 1994). While arsenate is strongly sorbed by soils under aerobic conditions, it is rapidly desorbed as the system becomes anaerobic. Once it is desorbed, arsenate can be reduced to arsenite, which exhibits greater mobility in soils (McGeehan 1996). Transformations between the various oxidation states and species of arsenic occur as a result of biotic or abiotic processes (Bhumbla and Keefer 1994). While degradation of an organic compound is typically considered complete mineralization, in the case of organic arsenic compounds, the element arsenic itself cannot be degraded. However, the organic portion of the molecule can be metabolized (Woolson 1976). Organic arsenical pesticides, such as MSMA, DSMA, and DMA, do not degrade by hydrolysis or by soil photolysis (EPA 2006).

Environmental partitioning: Arsenic in soil may be transported by wind or in runoff or may leach into the subsurface soil. However, because many arsenic compounds tend to partition to soil or sediment under oxidizing conditions, leaching usually does not transport arsenic to any great depth (EPA 1982a; Moore et al. 1988; Pantsar-Kallio and Manninen 1997; Welch et al. 1988). Arsenic is largely immobile in agricultural soils; therefore, it tends to concentrate and remain in upper soil layers indefinitely. Downward migration has been shown to be greater in a sandy soil than in a clay loam (Sanok et al. 1995). Arsenic from lead arsenate that was used for pest control did not migrate downward below $20 \mathrm{~cm}$ in one fruit orchard; in another orchard, 15 years after sludge amendments and deep plowing, essentially all arsenic residues remained in the upper 40 $\mathrm{cm}$ of soil (Merwin et al. 1994). Leaching of arsenic in polluted wetland soil was low; leaching was correlated with the amount of dissolved organic matter in the soil (Kalbitz and Wennrich 1998). The effect of soil characteristics, namely $\mathrm{pH}$, organic matter content, clay content, iron oxide content, aluminum oxide content, and cation exchange capacity (CEC), on the adsorption of various metals, including the metalloid arsenic, to 20 Dutch surface soils was assessed by regression analysis (Janssen et al. 1997). The most influential parameter affecting arsenic adsorption was the iron content of the soil.

Transport and partitioning of arsenic in water depends upon the chemical form (oxidation state and counter ion) of the arsenic and on interactions with other materials present. Soluble forms move with the water, and may be carried long distances through rivers (EPA 1979). However, arsenic may be adsorbed from water onto sediments or soils, 
especially clays, iron oxides, aluminum hydroxides, manganese compounds, and organic material (EPA 1979, 1982a; Welch et al. 1988). Under oxidizing and mildly reducing conditions, groundwater arsenic concentrations are usually controlled by adsorption rather than by mineral precipitation. The extent of arsenic adsorption under equilibrium conditions is characterized by the distribution coefficient, $\mathrm{Kd}$, which measures the equilibrium partitioning ratio of adsorbed to dissolved contaminant. The value of Kd depends strongly upon the $\mathrm{pH}$ of the water, the arsenic oxidation state, and the temperature. In acidic and neutral waters, $\mathrm{As}(\mathrm{V})$ is extensively adsorbed, while As (III) is relatively weakly adsorbed. Trivalent inorganic arsenic exists predominantly as arsenous acid $\left(\mathrm{H}_{3} \mathrm{AsO}_{3}\right)$ at environmental $\mathrm{pH}$ and is not strongly adsorbed to suspended solids and sediments in the water column.

Pentavalent inorganic arsenic exists predominantly as $\mathrm{H}_{2} \mathrm{AsO}_{4}$ and $\mathrm{HAsO}_{4}{ }^{2-}$ in most environmental waters, which has considerably greater adsorption characteristics than arsenous acid. While in acidic and neutral waters, $\mathrm{As}(\mathrm{V})$ is more strongly adsorbed relative to As(III), in high-pH waters $(\mathrm{pH}>9)$ aquifer $\mathrm{Kd}$ values are considerably lower for both oxidation states (Mariner et al. 1996). Sediment-bound arsenic may be released back into the water by chemical or biological interconversions of arsenic species. Arsenic enters rivers from where mining operations occurred and is transported downstream, moving from water and sediment into biofilm (attached algae, bacterial, and associated fine detrital material), and then into invertebrates and fish. The source of arsenic in the water column may be resuspended sediment. While arsenic bioaccumulates in animals, it does not appear to biomagnify between tropic levels (Eisler 1994; Farag et al. 1998; Williams et al. 2006).

Terrestrial plants may accumulate arsenic by root uptake from the soil or by absorption of airborne arsenic deposited on the leaves, and certain species may accumulate substantial levels (EPA 1982b). Yet, even when grown on highly polluted soil or soil naturally high in arsenic, the arsenic level taken up by the plants is comparatively low (Gebel et al. 1998b; Pitten et al. 1999). Kale, lettuce, carrots, and potatoes were grown in experimental plots surrounding a wood preservation factory in Denmark where waste wood was incinerated to investigate the amount and pathways for arsenic uptake by plants (Larsen et al. 1992). On incineration, the arsenate in the wood preservative was partially converted to arsenite; the arsenic emitted from the stack was primarily particle bound. Elevated levels of inorganic arsenic were found in the test plants and in the soil around the factory

\section{Regulatory}

MCL (Drinking Water): $0.01 \mathrm{mg} / \mathrm{L}$ 
Universal Treatment Standards (UTS): $1.4 \mathrm{mg} / \mathrm{l}^{2}$ in wastewater standards (Arsenic)

5.0 mg/L TCLP nonwastewater standard (Arsenic)

EPA Rf D: $0.0003 \mathrm{mg} / \mathrm{kg}$-day

EPA RfC: None

PRG: residential soil mg/kg industrial soil $\mathrm{mg} / \mathrm{kg}$ ambient air $\mathrm{ug} / \mathrm{m} \quad$ tap water $\mathrm{ug} / \mathrm{L}$ $\begin{array}{llll}0.39 & 2.7 & 0.00045 & 0.045\end{array}$

\section{Remediation}

The practice of liming to remediate contaminated soils and mine tailings has the potential to mobilize arsenic. Experiments performed by Jones et al. (1997) indicate that the increased mobility appears to be consistent with the $\mathrm{pH}$ dependence of sorption reactions of arsenic on iron oxide minerals rather than dissolution-precipitation reactions involving arsenic. They recommend that remediation of acidic mine tailings or other arseniccontaminated soils be carefully evaluated with respect to potential arsenic mobilization, especially at contaminated sites hydraulically connected to surface or groundwater.

\section{References}

Aurillo AC, Mason RP, Hemond HF (1994) Speciation and fate of arsenic in three lakes of the Aberjona Watershed. Environ Sci Tech 28(4):577-585.

Benson AA (1989) Arsonium compounds in algae. Proc Natl Acad Sci USA 86:6131-6132.

Braman RS, Foreback CC. (1973) Methylated forms of arsenic in the environment. Science 182:1247-1249.

Davis A, Ruby MV, Bloom M, et al. (1996) Mineralogic constraints on the bioavailability of arsenic in smelter-impacted soils. Environ Sci Technol 30(2):392-399.

Edmonds JS, Francesconi KA. (1987) Transformations of arsenic in the marine environment. Experientia 43:553-557.

Eisler R (1994) A review of arsenic hazards to plants and animals with emphasis on fishery and wildlife. In: Nriagu JO, ed. Arsenic in the environment: Part II: Human health and ecosystem effects. New York, NY: John Wiley \& Sons, Inc, pp. 185-259. 
EPA. (1982a) Exposure and risk assessment for arsenic. Washington, DC: U.S. Environmental Protection Agency, Office of Water Regulations and Standards. PB85221711. EPA440485005. 1.1 4.68.

EPA. (1982b) Inductively coupled plasma-atomic emission spectrometric method for trace element analysis of water and wastes - method 200.7. Cincinnati, OH: U.S. Environmental Protection Agency, Environmental Monitoring and Support Laboratory.

EPA. (1984) Health Assessment Document for Arsenic. Research Triangle Park, NC: U.S. Environmental Protection Agency. EPA600823021F.

EPA. (2006) Revised reregistration eligibility decision for MSMA, DSMA, CAMA, and cacodylic acid, August 10, 2006. Washington, DC: U.S. Environmental Protection Agency. http://www.epa.gov/oppsrrd1/REDs/organic_arsenicals_red.pdf. September 12, 2006

Farago ME (1997) Arsenic in the marine environment. In: Gianguzza A, Pelizzetti E, Sammartano S, eds. Marine chemistry. Netherlands: Kluwer Academic Publishers, pp. 275-291.

Farag AM, Woodward DF, Goldstein JN, et al. (1998) Concentrations of metal associated with mining waste in sediments, biofilm, benthic macroinvertebrates, and fish from the Coeur d'Alene River basin, Idaho. Arch Environ Contam Toxicol 34:119-127.

Gebel TW, Suchenwirth RHR, Bolten C, et al. 1998b. Human biomonitoring of arsenic and antimony in case of an elevated geogenic exposure. Environ Health Perspect 106(1):33-39.

Janssen RPT, Peijnenburg WJGM, Posthuma L, et al. (1997) Equilibrium partitioning of heavy metals in Dutch field soils: I. Relationship between metal partition coefficients and soil characteristics. Environ Toxicol Chem 16(12):2470-2478.

Jones CA, Inskeep WP, Neuman DR. (1997) Arsenic transport in contaminated mine tailings following liming. J Environ Qual 26(2):433-439.

Kalbitz K, Wennrich R. (1998) Mobilization of heavy metals and arsenic in polluted wetland soils and its dependence on dissolved organic matter. Sci Total Environ 209:2739.

Larsen EH, Moseholm L, Nielsen MM. (1992) Atmospheric deposition of trace elements around point sources and human health risk assessment: II. Uptake of arsenic and chromium by vegetables grown near a wood preservation factory. Sci Total Environ 126(3):263-275.

Mariner PE, Holzmer FJ, Jackson RE, et al. (1996) Effects of high pH on arsenic mobility in a shallow sandy aquifer and on aquifer permeability along the adjacent shoreline, 
Commencement Bay Superfund Site, Tacoma, Washington. Environ Sci Tech 30(5):16451651.

Merwin I, Pruyne PT, Ebel JG, et al. (1994) Persistence, phytotoxicity, and management of arsenic, lead and mercury residues in old orchard soils of New York State.

Chemosphere 29(6):1361-1367.

McGeehan SL. (1996) Arsenic sorption and redox reactions: Relevance to transport and remediation. J Environ Sci Health A31(9):2319-2336.

Moore JN, Ficklin WH, Johns C. (1988) Partitioning of arsenic and metals in reducing sulfidic sediments. Environ Sci Technol 22:432-437.

Pantsar-Kallio M, Manninen PKG. (1997) Speciation of mobile arsenic in soil samples as a function of pH. Sci Total Environ 204(2):193-200.

Pitten F, Müller G, König P, et al. (1999) Risk assessment of former military base contaminated with organoarsenic-based warfare agents: Uptake of arsenic by terrestrial plants. Sci Total Environ 226:237-245.

Redman AD, Macalady DL, Ahmann D. (2002) Natural organic matter affects arsenic speciation and sorption onto hematite. Environ Sci Technol 36:2889-2896.

Rhine ED, Phelps CD, Young LY. (2006) Anaerobic arsenite oxidation by novel denitrifying isolates. Environ Microbiol 8(5):899-908.

Roberts SM, Munson JW, Lowney YW, et al.(2007) Relative oral bioavailablity of arsenic from contaminated soils measured in the cynomolgus monkey. Toxicol Sci 95(1):281-288.

Ruby MV, Schoof R, Brattin W, et al. (1999) Advances in evaluating the oral bioavailability of inorganics in soil for use in human health risk assessment. Environ Sci Technol 33(21):3697-3705.

Sanders JG, Riedel GF, Osmann RW. (1994) Arsenic cycling and its impact in estuarine and coastal marine ecosystems. In: Nriagu JO, ed. Arsenic in the environment, part I: Cycling and characterization. New York, NY: John Wiley \& Sons, Inc., 289-308.

Sanok WJ, Ebel JG, JR, Manzell KL, et al. (1995) Residues of arsenic and lead in potato soils on long island. Chemosphere 30(4):803-806.

Scudlark JR, Church TM. (1988) The atmospheric deposition of arsenic and association with acid precipitation. Atmos Environ 22(5):937-943. 
Scudlark JR, Conko KM, Church TM. (1994) Atmospheric wet deposition of trace elements to Chesapeake Bay: CBAD study year 1 results. Atmos Environ 28(8):1487-1498.

Tamaki S, Frankenberger WT. (1992) Environmental biochemistry of arsenic. Rev Environ Contam Toxicol 124:79-110.

Wakao N, Koyatsu H, Komai Y, et al. (1988) Microbial oxidation of arsenite and occurrence of arsenite-oxidizing bacteria in acid mine water from a sulfur-pyrite mine. Geomicrobiol J 6:11-24.

Welch AH, Lico MS, Hughes JL. (1988) Arsenic in groundwater of the western United States. Ground Water 26(3):333-347.

Williams L, Schoof RA, Yager JW, et al. (2006) Arsenic bioaccumulation in freshwater fishes. Hum Ecol Risk Assess 12:904-923.

Woolson EA. (1976) Generation of dimethyl arsine from soil. 1976 Meeting of the Weed Science Society of America. February 3-5, No. 218. 


\title{
Sodium Cyanide 143-33-9 (Cyanide)
}

\section{ENVIRONMENTAL TRANSPORT, DISTRIBUTION, AND TRANSFORMATION}

\begin{abstract}
Air: Most cyanide in the atmosphere exists almost entirely as hydrogen cyanide gas, although small amounts of metal cyanides may be present as particulate matter in the air (EPA 1984). Hydrogen cyanide is very resistant to photolysis at wavelengths of normal sunlight (EPA 1979). The most important reaction of hydrogen cyanide in air is the reaction with photochemicallygenerated hydroxyl radicals and subsequent rapid oxidation to carbon monoxide (CO) and nitric oxide (NO); photolysis and reaction with ozone are not important transformation processes, and reaction with singlet oxygen $(\mathrm{O} 1 \mathrm{D})$ is not a significant transformation process except at stratospheric altitudes where singlet oxygen is present in significant concentrations (Cicerone and Zellner 1983). The rate of hydroxyl radical reaction with hydrogen cyanide in the atmosphere depends on the altitude, and the rate of the reaction is at least an order of magnitude faster at lower tropospheric altitudes $(0-8 \mathrm{~km})$ than at upper tropospheric altitudes $(10-12 \mathrm{~km})($ Cicerone and Zellner 1983). Based on a reaction rate constant of 3x10-14 cm3/(molecule-sec) at $25^{\circ} \mathrm{C}$ (Fritz et al. 1982) and assuming an average hydroxyl radical concentration of $5 \times 105$ molecules $/ \mathrm{cm} 3$, the residence time for the reaction of hydrogen cyanide vapor with hydroxyl radicals in the atmosphere is $\approx 2$ years. This value compares well with the atmospheric residence time derived by Cicerone and Zellner (1983) of approximately 2.5 years, with a range of 1.3-5.0 years, depending on the hydroxyl radical concentrations assumed. Using the equation $\mathrm{t}^{1} / 2=0.693 \tau$ for converting residence time $(\tau)$ to half-life ( $\left.t \frac{1}{2}\right)$ (Lyman 1982) and an estimated atmospheric residence time for hydrogen cyanide of 2-3 years, and assuming first-order kinetics for the reaction of hydrogen cyanide with hydroxyl radicals, an atmospheric half-life of 1.4- 2.9 years can be calculated for hydrogen cyanide.
\end{abstract}

Water: Cyanide occurs most commonly as hydrogen cyanide in water, although it can also occur as the cyanide ion, alkali and alkaline earth metal cyanides (potassium cyanide, sodium cyanide, calcium cyanide), relatively stable metallocyanide complexes (ferricyanide complex $\left[\mathrm{Fe}(\mathrm{CN})_{6}\right]^{-3}$ ), moderately stable metallocyanide complexes (complex nickel and copper cyanide), or easily decomposable metallocyanide complexes (zinc cyanide $\left[\mathrm{Zn}(\mathrm{CN})_{2}\right]$, cadmium cyanide $\left[\mathrm{Cd}(\mathrm{CN})_{2}\right]$ ). The environmental fate of these cyanide compounds varies widely (EPA 1979).

In water, hydrogen cyanide and cyanide ion exist in equilibrium with their relative concentrations primarily dependent on $\mathrm{pH}$ and temperature. At $\mathrm{pH}<8,>93 \%$ of the free cyanide in water will exist as undissociated hydrogen cyanide (EPA 1978). Hydrogen cyanide can be hydrolyzed to formamide, which is subsequently hydrolyzed to ammonium and formate ions (EPA 1979). However, the relatively slow rates of hydrolysis reported for hydrogen cyanide in acidic solution (Krieble and McNally 1929; Krieble and Peiker 1933) and of cyanides under alkaline conditions (Wiegand and Tremelling 1972) indicate that hydrolysis is not competitive with volatilization and biodegradation for removal of free cyanide from ambient waters (EPA 1979).

Sediment and Soil: Analogous to the fate of cyanides in water, it is predicted that the fate of cyanides in soil would be dependent on cyanide concentrations, $\mathrm{pH}$, temperature, metal content, concentration of microbes, availability of nutrients, and acclimation of microbes. Cyanide may occur as hydrogen cyanide, alkali metal salts, or as immobile metallocyanide complexes. In soil, cyanide present at low concentrations would biodegrade under aerobic conditions with the initial formation of ammonia, which would be converted to nitrite and nitrate in the presence of nitrifying bacteria. Under anaerobic conditions, the cyanides ion will denitrify to gaseous nitrogen (Richards and Shieh 1989). Upper limits of 200 and $2 \mathrm{ppm}(\mathrm{mg} / \mathrm{kg} \mathrm{CN})^{-}$, respectively, have been reported for uninhibited aerobic and anaerobic biodegradation of cyanide in soil (Fueller 1985); however, these limits have not been confirmed in other studies (Thomas and Lester 1993). Cyanide ions in 
soil are not involved in oxidation-reduction reactions but may undergo complexation reactions with metal ions in soil (EPA 1978).

Environmental partitioning: Because hydrogen cyanide is a gas and has a relatively slow degradation rate in air, the atmosphere will be the ultimate sink for this compound. Almost all of the hydrogen cyanide released to the atmosphere remains in the lower altitudes (troposphere); only $2 \%$ of tropospheric hydrogen cyanide is transferred to the stratosphere (Cicerone and Zellner 1983). Cyanide has the potential to be transported over long distances from its emission source. Despite higher water solubility at saturated pressure, the removal of hydrogen cyanide by rainwater appears to be a negligible partitioning pathway (Cicerone and Zellner 1983). Because hydrogen cyanide is a gas, its removal from air by dry deposition is also likely to be negligible. However, metal cyanide particles, particularly water-soluble cyanide particles, are expected to be removed from the air by both wet and dry deposition.

Volatilization and sorption are the two physical processes that contribute to the loss of cyanide from water. At $\mathrm{pH}<9.2$, most of the free cyanide in solution should exist as hydrogen cyanide, a volatile cyanide form (EPA 1978). On the basis of Henry's law constant and the volatility characteristics associated with various ranges of Henry's law constant (Thomas 1982), volatilization is a significant and probably dominant fate process for hydrogen cyanide in surface water (EPA 1992). The most common alkali metal cyanides (e.g., sodium and potassium cyanide) may also be lost from surface water primarily through volatilization; whereas, the sparingly soluble metal cyanides such as copper (I) cyanide are removed from water predominantly by sedimentation and biodegradation (EPA 1992). Variations in the volatilization rate are expected because this process is affected by several parameters including temperature, $\mathrm{pH}$, wind speed, and cyanide concentration (EPA 1979). EPA (1979) summarized results of a laboratory study that indicated that the volatilization half-life of hydrogen cyanide from solutions at concentrations of $25-200 \mu \mathrm{g} / \mathrm{L}$ ranged from 22 to 110 hours. First-order kinetics were observed. In outdoor experiments with moderate winds, the rate of hydrogen cyanide loss increased by a factor of 2 2.5. In a study to evaluate the effect of cyanide on biochemical oxidation, there was a $50 \%$ loss of $6 \mathrm{ppm}(\mathrm{mg} / \mathrm{L})$ cyanide in river water kept in open biochemical oxygen demand bottles (without aeration) at $\mathrm{pH} 7.4$ within $\approx 10$ days (Ludzack et al. 1951). When the bottles were aerated (rate of aeration not given), $50 \%$ loss occurred in only $\approx 10$ hours. The kinetics of the rate of loss due to volatilization were not rigorously investigated. The volatilization rate was $\mathrm{pH}$-dependent, with the rate faster at a lower $\mathrm{pH}$. Data indicated that cyanide volatilization is a more important fate process than cyanide loss due to chemical and biodegradation reactions (Ludzack et al. 1951; Raef et al. 1977a). Because volatilization is not an important fate process for cyanide in groundwater, cyanide would be expected to persist for considerably longer periods of time in underground aquifers than in surface water.

\section{Regulatory}

Canada Drinking Water Standards and Criteria (MAC): $200 \mathrm{ug} / \mathrm{L}$

Canadian Water Quality Guidelines for the Protection of Aquatic Life: 5 ug/L

MCL (Drinking Water): $0.2 \mathrm{mg} / \mathrm{L}(\mathrm{CN})$

Universal Treatment Standards (UTS) for CN totals: $1.2 \mathrm{mg} / \mathrm{l}^{2}$ in wastewater standards

$590 \mathrm{mg} / \mathrm{L}$ in nonwastewater standards 
EPA RfD: 0.04 mg/kg-day

EPA RfC: $3.0 \quad \mathrm{mg} / \mathrm{m}^{3}$

PRG: residential soil $\mathrm{mg} / \mathrm{kg}$ industrial soil $\mathrm{mg} / \mathrm{kg} \quad$ ambient air $\mathrm{ug} / \mathrm{m} \quad$ tap water $\mathrm{ug} / \mathrm{L}$
11
35
3.1
6.2

\section{Remediation}

The biodegradation of cyanides has been investigated, with varying results, for several industrial processes, and additional research in this area would be valuable. While investigations of the potential for microbial species found in mineral processing waste waters demonstrate effective removal of cyanide, metal complexed cyanide, and thiocyanate (Boucabeille 1994; EPA 1994), complex cyanides did not appear amenable to biodegradation at gasworks sites (Thomas and Lester 1993). Application of formaldehyde to electroplating waste under basic conditions can convert the cyanide anion to substituted acetates in addition to recovering copper and silver as free metals with formaldehyde reduction (Tucker and Carson 1985). Calcium or sodium polysulfide treatment converts some cyanide wastes into less toxic thiocyanate (Higgins and Desher 1988). These examples suggest that typical treatments involve the decomposition of cyanides to less toxic compounds by physical or chemical processes. More than $97 \%$ of cyanide is typically removed from waste waters by alkaline chlorination, electrolysis, or ozonation process. Cyanide from some wastes can be removed by ion-exchange resins. After using an appropriate treatment method such as those described above, cyanide wastes may be disposed of in a secured sanitary landfill (Grosse 1986; Higgins and Desher 1988; Tucker and Carson 1985). Disposal by injection of high-pH cyanide wastes into sandstone was investigated by Scrivner et al. (1986). Currently, the injection of waste water containing hydrogen cyanide and cyanide compounds through underground injection is a major method for disposal of these wastes.

\section{References}

Boucabeille C, Bories A, Ollivier P, et al. (1994) Microbial degradation of metal complexed cyanides and thiocyanate from mining wastewaters. Environ Poll 84(1):59-67.

Cicerone RJ, Zellner R (1983) The atmospheric chemistry of hydrogen cyanide (HCN). J Geophys Res 88:10689-10696.

EPA (1978) Reviews of the environmental effects of pollutants. V. Cyanide. Cincinnati, OH: U.S. Environmental Protection Agency Health Effects Research Laboratory, Office of Research and Development. PB289920. 
EPA (1979) Cyanides. In: Water-related environmental fate of 129 priority pollutants. Vol. 1. Washington, DC: U.S. Environmental Protection Agency, Office of Water Planning and Standards, Office of Water and Waste Management. EPA440479029a. PB80204373 pp. 12.1-12.12.

EPA (1992) U.S. Environmental Protection Agency. Fed Regist 57:26248.

EPA. (1994) Technical report: Treatment of cyanide heap leaches and tailings. Washington, DC: U.S. Environmental Protection Agency, Office of Solid Waste. EPA530R94037. PB94201837.

Fueller WH (1985) Cyanides in the environment with particular attention to the soil. In: Van Zyl D, ed. Cyanide and the environment: Proceedings of a conference Tucson, Arizona December 11-14, 1984: Volume 1. Fort Collins, CO: Colorado State University, pp. $19-46$.

Grosse DW (1986) Treatment technologies for hazardous wastes part IV. A review of alternative treatment processes for metal-bearing hazardous waste streams. J Air Pollut Control Assoc 36:603-614.

Higgins TE, Desher DP (1988) Metal finishing and processing. J Water Pollut Control Fed 60:904-909.

Krieble VK, McNally JG (1929) The hydrolysis of hydrogen cyanide by acids. J Am Chem Soc 51:3368-3375.

Krieble VK, Peiker AL (1933) The hydrolysis of hydrogen cyanide by acids II. J Am Chem Soc 55:2326-2331.

Lyman, W (1982) Atmospheric residence time. In: Lyman WJ, Reehl WF, Rosenblatt DH, eds. Handbook of chemical property estimation methods: Environmental behavior of organic compounds. New York, NY: McGraw Hill Book Company, pp. 10.2-10.33.

Ludzack FJ, Moore WA, Krieger HL, et al. (1951) Effect of cyanide on biochemical oxidation in sewage and polluted water. Sewage Ind Wastes 23:1298-1307.

Raef SF, Characklis WG, Kessick MA, et al. (1977) Fate of cyanide and related compounds in aerobic microbial systems--II. Microbial degradation. Water Res 11:485-492.

Scrivner NC, Bennett KE, Pease RA, et al. (1986) Chemical fate of injected wastes. Ground Water Monit Rev 6:53-58. 
Thomas AO, Lester JN (1993) The microbial remediation of former gasworks sites. A review. Environ Technol 14(1):1-24.

Thomas RG. (1982) Volatilization from water. In: Lyman WJ, Reehl, WF, Rosenblatt DH, eds. Handbook of chemical property estimation methods: Environmental behavior of organic compounds. New York, NY: McGraw-Hill Book Co., pp 15-16.

Tucker SP, Carson GA. (1985) Deactivation of hazardous chemical wastes. Environ Sci Technol 19:215-220.

Wiegand GH, Tremelling M (1972) The kinetics and mechanism of the decomposition of potassium cyanide in aqueous alkaline medium. Hydrolysis of the simplest nitrile. J Org Chem 37(6):914-916. 


\title{
Sulfur Dioxide 7446-09-5
}

\section{ENVIRONMENTAL TRANSPORT, DISTRIBUTION, AND TRANSFORMATION}

\begin{abstract}
Air: Wet deposition is, in general, much more easily measured than is dry deposition. Routine measurement of wet deposition is determined from sulfate concentration in precipitation samples and precipitation amount. Typically, the removal rate for particulate sulfate is of the order of $40 \%$ per $\mathrm{hr}$, and for sulfur dioxide, an order of magnitude less. The overall efficiency of wet removal depends on many factors: precipitation type, intensity, duration, frequency, the relative amounts of sulfur dioxide and sulfate present, and the size distribution of particulate sulfate
\end{abstract}

Direct surface uptake of sulfur dioxide is the most important dry removal process for atmospheric sulfur; good sinks /include/ oceans $(\mathrm{pH}=8)$, other non acidic moist surfaces, and some crops and forest species at certain growth stages; where as dry, snow covered surfaces and soils, for example, are less efficient (Monitoring and Assessment Research Centre 1978).

Water: Although snow covered surfaces are inefficient receptors of gaseous and particulate sulfur compound, the spring melt of the accumulated winter snowpack can result in rapid, short term inputs of high sulfate, low $\mathrm{pH}$ water to freshwater systems with resulting disastrous effects on fish (Monitoring \& Assessment Research Centre 1978).

Environmental Partitioning: When released into the environment, sulfur dioxide moves into the air. In the air, it can be converted to sulfuric acid, sulfur trioxide, and sulfates. Sulfur dioxide dissolves in water. Once dissolved in water, sulfur dioxide can form sulfurous acid. Sulfur dioxide can be absorbed into the soil, but we don't know if or how it moves in soil (ATSDR 1998).

\section{Regulations}

IDLH: $100 \mathrm{ppm}$

Acute MRL: 10ppm

MCL (Drinking Water): None

Universal Treatment Standards (UTS): none 
Groundwater Water Quality Criterion : none ug/L

EPA Rf D: none mg/kg-day

EPA RfC: none $\mathrm{mg} / \mathrm{m}^{3}$

\section{Remediation}

Ventilate area of leak to disperse gas. If sulfur dioxide is in gaseous form, stop the flow of gas. Remove to safe place in open air and allow emptying. If in the liquid form, allow to vaporize (Mackison et al. 1981).

Approach release from upwind. Stop or control the leak, if this can be done without undue risk. Use water spray to cool and disperse vapors and protect personnel. Control runoff and isolate discharged material for proper disposal. Releases may require isolation or evacuation (Fire Protection Guide 1997).

\section{References}

Agency for Toxic Substances and Disease Registry (ATSDR). 1998. Toxicological Profile for Sulfur Dioxide. Atlanta, GA: U.S. Department of Health and Human Services, Public Health Service.

Fire Protection Guide to Hazardous Materials. 12 ed. Quincy, MA: National Fire Protection Association, 1997. p. 49-123.

Mackison, F. W., R. S. Stricoff, and L. J. Partridge, Jr. (eds.). (1981) NIOSH/OSHA Occupational Health Guidelines for Chemical Hazards. DHHS (NIOSH) Publication No. 81-123 (3 VOLS). Washington, DC: U.S. Government Printing Office, Jan. p. 3.

Monitoring and Assessment Research Centre; Report \#7 p.13-14, 21 (1978). 


\title{
Sulfur Trioxide $7446-11-9$
}

\section{ENVIRONMENTAL TRANSPORT, DISTRIBUTION, AND TRANSFORMATION}

\begin{abstract}
Air: Sulfuric acid is formed in the atmosphere from sulfur dioxide. Both gaseous and aqueous phase reactions can occur, with gas phase reactions accounting for only about $5 \%$ of the sulfur dioxide oxidized during the summer (Pienaar and Helas 1996). In the process of sulfuric acid formation, sulfur trioxide is formed. After sulfur trioxide is formed, it rapidly reacts with water vapor to form sulfuric acid, so that processes that form sulfur trioxide in moist atmospheres are equivalent to the formation of sulfuric acid (Pienaar and Helas 1996). In the gaseous phase, substances that react with sulfur dioxide resulting in the production of sulfuric acid include $\mathrm{O}, \mathrm{HO} \bullet, \mathrm{HO}_{2} \bullet$, and $\mathrm{CH}_{2} \mathrm{O}_{3} \bullet$. Substances that result in the oxidation of sulfur dioxide in the aqueous phase include ozone and hydrogen peroxide. Metal ions including $\mathrm{CI}^{-} \mathrm{O}_{4}{ }^{2-}, \mathrm{VO}_{2+}, \mathrm{Fe}^{2+}, \mathrm{Fe}^{3+}, \mathrm{Mn}^{2+}$, and $\mathrm{Ni}^{2+}$ could also directly oxidize $\mathrm{SO}_{3}{ }^{2-}$, or catalyze $\mathrm{SO}_{3}{ }^{2-}$ oxidation by molecular oxygen. In a study using electron spin resonance, spin trapping, and high-performance liquid chromatography, Shi (1994) found that reactions of $\mathrm{SO}_{3}{ }^{2-}$ with $\mathrm{NO}_{2}$-also generate $\mathrm{SO}_{3}$ • radicals. The major reactions that form sulfuric acid from sulfur dioxide require light. Therefore, levels of sulfuric acid in the atmosphere show both seasonal and diurnal variations.
\end{abstract}

The production of sulfuric acid from sulfur dioxide can become limited if the concentrations of oxidants are less than the concentrations of sulfur dioxide. Oxidant limitations of the formation of sulfuric acid were shown in a study of sulfur dioxide and hydrogen peroxide concentrations over Columbus, Ohio; the study authors suggested that similar conditions exist over a large area of the eastern United States (Kleinman and Daum 199 1). A deficiency of hydrogen peroxide relative to sulfur dioxide was also shown in a study completed at Whiteface Mountain, NY (Dutkiewicz et al. 1995). These results suggest that there would be a nonlinear relationship between sulfur dioxide emissions and downwind acid precipitation in the northeastern United States where aqueous-phase oxidation by hydrogen peroxide is the principal mechanism for forming sulfuric acid. The aqueous oxidation of sulfur dioxide to sulfuric acid in precipitation has been estimated to account for 50-80\% of the sulfuric acid found in precipitation (Fung et al. 1991).

Water: Sulfuric acid is very corrosive and is highly toxic to aquatic and terrestrial life at low concentrations. In water, sulfuric acid dissociates. The sulfate anion may associate with other cations including calcium, magnesium, and aluminum. Sulfur that is in water may be oxidized to sulfuric acid by sulfur bacteria (Thiobacilli) that use sulfur to obtain energy for growth (Takeuchi and Suzuki 1994). Sulfate in water can also be reduced. 
Because sulfur dioxide and sulfate are transformed through similar pathways in water, the effect of sulfur on aquatic systems is not dependent on the chemical or physical form of deposition (wet or dry) (EPA 1985). In the surface layer of the ocean the sulfate anion may be formed from dissolved sulfur dioxide, which is transformed to sulfurous acid $(\mathrm{H}, \mathrm{SO}$,$) and subsequently oxidized. Because of the relative lack of salt in freshwater, the$ oxidation of sulfur dioxide to sulfate is less likely to occur in freshwater. In the depths of the ocean, sulfate is reduced to sulfur dioxide, sulfur, and hydrogen sulfide by bacteria (Kellogg et al. 1972).

Sediment and Soil: When released into the soil, this material may leach into groundwater. When released into the air, this material may be removed from the atmosphere to a moderate extent by wet deposition. When released into the air, this material may be removed from the atmosphere to a moderate extent by dry deposition. The ions (sulfate, hydrogen) can adsorb to soil particles or be converted to gases (EPA 1985). Anaerobic bacteria in sediments and soil can reduce sulfate to sulfur and hydrogen sulfide (Kellogg et al. 1972).

Transport and Partitioning: Factors that affect the dispersion of sulfur compounds and other air pollutants include the temperature and efflux velocity of the emissions, stack height, topography and the proximity of other buildings, and meteorology. Meteorological conditions that result in temperature inversions can result in the trapping of pollutants, which increases concentrations up to several hundred times the concentrations usually found (WHO 1979). Sulfates, including sulfuric acid, are removed from the air by both dry and wet deposition processes. Wet deposition processes including rainout (a process that occurs within the clouds) and washout (removal by precipitation below the clouds) contribute to the removal of sulfate from the atmosphere (Kellogg et al. 1972). In the stratosphere, sulfuric acid aerosols have lifetimes of about 14 and 2.4 days at altitudes of 15 and $20 \mathrm{~km}$, respectively (Kellogg et al. 1972). At cloud level, the residence time is about 6 days, with shorter residence times in surface air.

\title{
Regulatory-Sulfuric Acid
}

\author{
MCL (Drinking Water): None
}

IDLH: $15 \mathrm{mg} / \mathrm{m}^{3}$

Universal Treatment Standards (UTS): None

MRL: None 
EPA Rf D: None

EPA RfC: None

PRG: residential soil $\mathrm{mg} / \mathrm{kg}$ industrial soil $\mathrm{mg} / \mathrm{kg} \quad$ ambient air ug/m $\quad$ tap water ug/L None None None None

\section{Remediation}

Spent sulfuric acid can often be reprocessed to obtain a product of virgin quality. Enormous amounts of spent sulfuric acid are reprocessed since most of the sulfuric acid used for industrial processes acts only as a reagent and does not form part of the final product; one exception is the fertilizer industry (IARC 1992). It has been suggested that waste sulfuric acid can also be recycled using sulfate-reducing bacteria to produce hydrogen sulfide (Stucki et al. 1993).

It is not recommended that sulfuric acid or sulfur trioxide be placed in a landfill. Environmental regulatory agencies should be consulted for acceptable disposal practices (HSDB 1998). Sulfuric acid has been disposed of by being placed in sealed containers and by being absorbed in vermiculite, dry sand, or earth. Sulfuric acid may also be diluted and then neutralized. One method of neutralization is to add the acid slowly to a solution of soda ash and slaked lime, and to then flush with a large volume of water. Once sulfuric acid is diluted, and neutralized it can be discharged to a sewer. When diluting, the acid should always be added to a large volume of water because the heat released when a small bolus of water is added can cause the water to turn to steam, and the resulting effervescence can splatter the acid.

\section{References}

Budavari S, ed. 1989. The Merck index. 1 lth ed. Rahway, NJ: Merck and Co., Inc. 89538954.

Dutkiewicz VA, Burkhard EG, Husain L. 1995. Availability of H,O, for oxidation of SO, in clouds in the northeastern United States Atmos Environ 29:3281-3292.

EPA. 1985. The acidic deposition phenomenon and its effects: Critical assessment document. United States Environmental Protection Agency, Office of Acid Deposition, Environmental Monitoring, and Quality Assurance, Washington, DC. EPA/60o/885/001. 
Fung CS, Misra PK, Bloxam R, et al. 1991. A numerical experiment on the relative importance of $\mathrm{H}, \mathrm{O}$, and o, in aqueous conversion of $\mathrm{SO}_{2}$ to $\mathrm{SO}_{4}$ 2-. Atmos Environ 25A:41l-423.

HSDB. 1998. Hazardous Substances Data Bank National Library of Medicine, Toxicology Information Program, Bethesda, MD. March 1998.

IARC. 1992. Occupational exposures to mists and vapours from sulfuric acid and other strong inorganic acids. IARC Monogr Eva1 Carcinogen Risk Chem Hum 5441-130.

Kellogg WW, Cadle RD, Allen ER, et al. 1972. The sulfur cycle. Science 175:587-596.

Kerminen V-M, Wexler AS 1996. The occurrence of sulfuric acid-water nucleation in plumes: Urban environment. Tellus 45B:65-82.

Kleinman LI, Daum PH. 1991. Oxidant limitation to the formation of $\mathrm{H} 2 \mathrm{SO} 2$ near a $\mathrm{SO} 2$ source region. Atmos Environ 25A:2023-2028.

Pienaar JJ, Helas G. 1996. The kinetics of chemical processes affecting acidity in the atmosphere. South African Journal of Science 92: 128-132.

Shi X. 1994. Generation of SO;- and OH radicals iu SO,'- reactions with inorganic environmental pollutants and its implications to SO,- toxicity. J Inorg Biochem 56:155165 .

Stucki G, Hanselmann KW, Hurzeler RA. 1993. Biological sulfuric acid transformation: Reactor design and process optimization. Biotechnol Bioeng 41:303-3 15.

Takeuchi TL, Suzuki I. 1994. Effect of pH on sulfite oxidation by Thiobacillus thiooxiduns cells with sulfurous acid or sulfur dioxide as a possible substrate. Journal of Bacterial 176:9 13-9 16. 


\section{Trimethylamine 75-50-3}

\section{ENVIRONMENTAL TRANSPORT, DISTRIBUTION, AND TRANSFORMATION}

Air: According to a model of gas/particle partitioning of semivolatile organic compounds in the atmosphere (Bidleman 1988), trimethylamine, which has a vapor pressure of $1.6 \mathrm{X}_{10} \mathrm{~mm} \mathrm{Hg}^{\mathrm{m}}$ at $25 \mathrm{deg} \mathrm{C}$ (Daubert and Danner 1989), is expected to exist solely as a gas in the ambient atmosphere. Gas-phase trimethylamine is degraded in the atmosphere by reaction with photochemically-produced hydroxyl radicals (SRC); the half-life for this reaction in air is estimated to be $9 \mathrm{hrs}$ (SRC), calculated from its rate

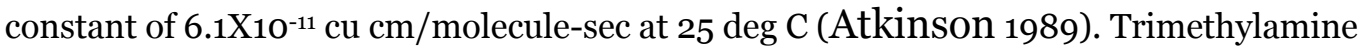
does not contain chromophores that absorb at wavelengths $>290 \mathrm{~nm}$ and therefore is not expected to undergo direct photolysis by sunlight (Lyman et al 1990).

Water: Based on a classification scheme (Swann et al 1983), an estimated Koc value of 29, determined from a measured log Kow of 0.16 (Hansch et al 1995) and a regressionderived equation (Lyman et al 1990), indicates that trimethylamine is not expected to adsorb to suspended solids and sediment (SRC). However, trimethylamine has a pKa of 9.8 (Perinn 1972) and should exist primarily as a cation under environmental conditions ( $\mathrm{pH}$ 5-9) (SRC). As a result, trimethylamine may have greater adsorption to suspended solids and sediment than its estimated Koc value indicates (SRC). Volatilization of trimethylamine from water surfaces will not be an important fate process, since cations do not volatilize (SRC). According to a classification scheme, an estimated BCF of 3(SRC), from its log Kow (Hansch et al 1995) and a regression-derived equation (Meylan et al 1999), suggests the potential for bioconcentration in aquatic organisms is low(SRC). Trimethylamine incubated in a marine sediment (Lowes Cove, ME) slurry underwent about 35\% removal in $12 \mathrm{hrs}$, as measured by production of $\mathrm{CO}_{2}$ and $\mathrm{CH}_{4}$ (King et al 1983). Degradation products formed under aerobic conditions include dimethylamine, formaldehyde, formate, and $\mathrm{CO}_{2}$ (Tate and Alexander 1976), while products formed under anaerobic conditions include dimethylamine, $\mathrm{NH}_{4}{ }^{+}$, and $\mathrm{CH}_{4}(\mathrm{Meiberg}$ and Harber 1978). Trimethylamine achieved 66-92\% of its theoretical BOD in 2 weeks using an activated sludge inoculum in the Japanese MITI test (Chemicals Evaluation Research Institute).

Soil/Sediment: Based on a classification scheme (Swann et al 1983), an estimated Koc value of 29(SRC) determined from a measured log Kow of 0.16(2) and a regressionderived equation (Lyman et al 1990), indicates that trimethylamine is expected to have very high mobility in soil (SRC). However, trimethylamine has a pKa of 9.8 (Perinn 1972) and should exist primarily as a cation under environmental conditions ( $\mathrm{pH}$ 5-9) 
(SRC). As a result, trimethylamine may have greater adsorption and less mobility than its estimated Koc value indicates since cations generally adsorb more strongly to soils containing organic carbon and clay than neutral species (Doucette 2000). Sorption coefficients for trimethylamine adsorption on montmorillonite, kaolinite and Flax Pond sediment (7\% clay, 2.8\% OM; Long Island, NY) were 15, 2 and $7 \mathrm{ml} / \mathrm{g}$, respectively (Wang and Lee 1993). The trimethylamine cation adsorbed strongest to the negatively-charged montmorillonite via electrostatic interactions (Wang and Lee 1993). Volatilization of trimethylamine from moist soil surfaces will not be an important fate process because cationic trimethylamine does not volatilize. The potential for volatilization of trimethylamine from dry soil surfaces may exist (SRC) based upon a vapor pressure of 1,610 mm Hg at 25 deg C (Daubert and Danner 1989). Microbial production of dimethylamine from trimethylamine in soil was found to be greater under acidic conditions than at near neutral $\mathrm{pH}$ and greater under aerobic conditions than anaerobic conditions (Tate and Alexander 1976). Degradation products formed under aerobic conditions include dimethylamine, formaldehyde, formate, and $\mathrm{CO}_{2}$ (Meiberg and Harber 1978), while products formed under anaerobic conditions include dimethylamine, $\mathrm{NH}_{4}{ }^{+}$, and $\mathrm{CH}_{4}$ (Hippe et al 1977).

Biota: An estimated BCF of 3 was calculated for trimethylamine (SRC), using a log Kow of 0.16 (Hansch et al 1995) and a regression-derived equation (Meylan et al 1999). According to a classification scheme (Franke et al 1994), this BCF suggests the potential for bioconcentration in aquatic organisms is low.

\section{Regulations}

MCL (Drinking Water): none

Universal Treatment Standards (UTS): None

EPA RfD: none $\mathrm{mg} / \mathrm{kg}$-day

EPA RfC: $0.007 \mathrm{mg} / \mathrm{m} 3$

$\begin{array}{cccc}\text { PRG: residential soil mg/kg industrial soil mg/kg } & \text { ambient air ug/m3 } & \text { tap water ug/L } \\ 23 & 88 & 7.3 & 12\end{array}$

\section{Remediation}

Water spill: Add sodium bisulfate ( $\mathrm{NaHSO}_{4}$ ). If dissolved, in region of $10 \mathrm{ppm}$ or greater concentration, apply activated carbon at ten times the spilled amount. Use mechanical dredges or lifts to remove immobilized masses of pollutants and precipitates. 
Air release: Apply water spray or mist to knock down vapors. Vapor knockdown water is corrosive or toxic and should be diked for containment (Association of American Railroads).

\section{References}

Association of American Railroads; Bureau of Explosives. Emergency Handling of Hazardous Materials in Surface Transportation. Association of American Railroads, Pueblo, CO. 2005, p. 914-915.

Atkinson R; (1989) Kinetics and mechanisms of the gas-phase reactions of the hydroxyl radical with organic compounds. J Phys Chem Ref Data Monograph 1.

Bidleman T. F. (1988) Atmospheric processes. Envir. Sei. Technol. 22:361-367

Chemicals Evaluation Research Institute (Japan); Biodegradation and Bioaccumulation, Data of Existing Chemicals (Trimethylamine). Available from the Database query page at http://www.safe.nite.go.jp/english/kizon/KIZON_start_hazkizon.html as of Dec 13, 2007.

Daubert TE, Danner RP. (1989) Physical and Thermodynamic Properties of Pure Chemicals: Data Compilation. Design Inst Phys Prop Data, Amer Inst Chem Eng, NY, NY: Hemisphere Pub Corp, 5 Vol.

Doucette WJ; (2000) in Handbook of Property Estimation Methods for Chemicals. Boethling RS, Mackay D, eds. Boca Raton, FL: Lewis Publ pp. 141-188.

Franke, C., G. Studinger, G. Berger, S. Böhling, U. Bruckmann, D. Cohors-Fressenborg and U. Jöhncke (1994) The assessment of bioaccumulation. Chemosphere 29: 1501-14

Hansch, C., Leo, A., and Hoekman, D. (1995) Exploring QSAR. Hydrophobic, Electronic, and Steric Constants. ACS Prof Ref Book. Washington, DC: American Chemical Society, p. 3 .

Hippe, H.D. Caspari, K. Fiebig and G. Gottschalk. (1979) Engineering, operation and economics of methane gas production. ProcNatl. Acad. Sci. USA 76: 494-98.

King, GM, M. J. Klug, and D. R. Lovely, (1983) Metabolism of Acetate, Methanol, And Methylated Amines in Intertidal Sediments of Lowes Cove, Maine. 
Lyman, WJ, WF Reehl, and DH Rosenblatt (1990) Handbook of Chemical Property Estimation Methods. Washington, DC: American Chemical Society pp. 8-12.

Meiberg, J. B. M., and W. Harder. 1978. Aerobic and anaerobic metabolism of trimethylamine, dimethylamine and methylamine in Hyphomicrobium X. J. Gen. Microbiol. 106:265-276.

Meylan, WM; Howard, PH; and Boethling, RS (1999) Improved method for estimating bioconcentration/bioaccumulation factor from octanol/water partition coefficient. Environ. Toxicol. Chem. 18: 664-672 .

Perinn DD. (1972) Dissociation Constants of Organic Bases in Aqueous Solution. IUPAC Chem Data Ser: Suppl 1972. London, England: Buttersworth.

Swann, R.L., Laskowski, D.A., McCall, P.J., van der Kuy, K. and Dishburger, H.J. (1983) A rapid method for estimation of the environmental parameters octanol/water partition coefficient, soil sorption constant water to air ratio and water solubility. Residue Rev. 85, pp. 17-28. (http://toxnet.nlm.nih.gov/cgi-bin/sis/search/r?dbs+hsdb:@term+@rn+6o34-4).

Tate RL, Alexander M; (1976) Microbial formation and degradation of dimethylamine. Appl Environ Microbiol 31: 399-403.

Wang XC, Lee C; (1993) Adsorption and desorption of aliphatic amines, Mar Chem 44: 123. 


\section{Appendix D: TIC-Master Fact Sheet}




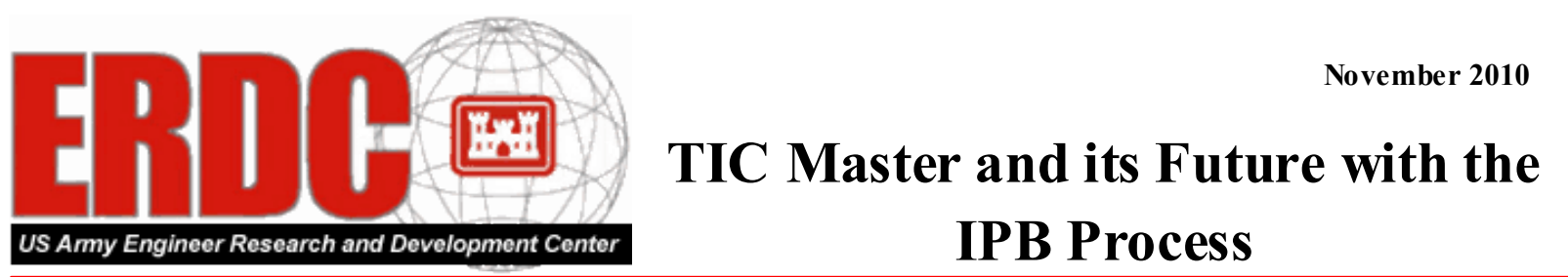

Toxic Industrial Che mical (TIC) exposures may result during military urban operations involving industrial or manufacturing facilities. Even if soldiers are adequately protected, TIC releases can affect neutral or friendly populations in the environs of an urban operation. A better understanding and mapping of the types of TICs that may be present in a battlefield from industrial sources is needed as an important part of an assessment tool that could be incorporated into the Intellige nce Preparation of the Battlefield (IPB) process.

TIC Database/GIS Platform Integration:

A database has been built that currently contains over 2,000 TICs, primarily from the International Task Force 40 and EPA Toxic Release Inventory (TRI). The database includes physical properties, hazard assessment information, and environmental effects and remediation information. A data model has been built to relate these chemicals and industrial types as listed as 6digit codes from the North American Industrial Classification System (NAICS). Data to relate the chemicals to the industries was queried from the TRI database. Analysis of the data allows for a crude estimation of the probability to encounter specific

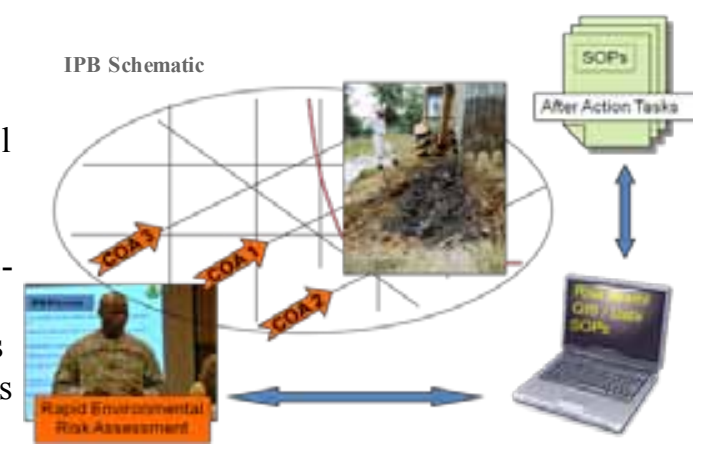
TICs at a particular industrial facility. A custom GIS standards based platform has been designed to specifically map industrial facilities within a designated region. The integration of the database and the GIS platform allows relevant information about the chemical(s) to be queried from the TIC database for a specific site and the mapping of areas of highest hazard probability. This technology offers a physical layout of industrial chemical hazards that can be the basis for the continuation of a more comprehensive effort to assess potential battlefields for TIC hazards in regions with less available data (OCONUS (Outside the Continental United States)).

\section{Test 5: Comparison of Two Facilities}

-Evaluated potential operation against 2 facilities, determined which presented least risk.

-Successfully determined industries from partial list of chemicals -Determined community risk (schools, population etc.)

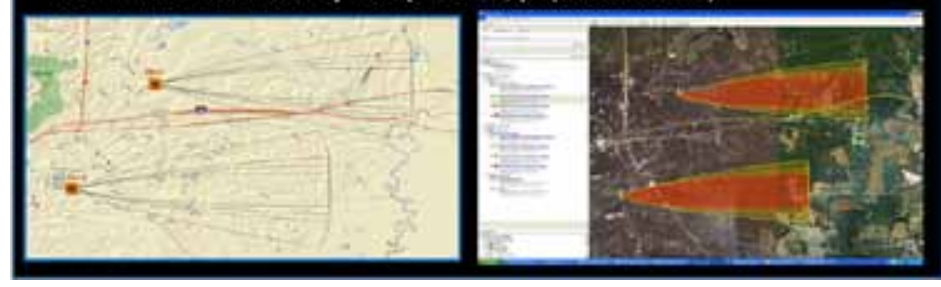

Developmental Testing:

A series of test scenarios were created by a semi-independent team to test the predictive aspects of the integrated geospatial system along with the TIC database. The tests were conducting using actual industrial facilities in the Vickburg Mississippi area using EPA Tier 2 data. There were five scenarios given to the test team that represented a broad spectrum of industries and hazardous situations. The industrial process, TIC re-

lease mechanism (depending on military action) and atmospheric conditions changed with each. Each scenario provided the geospatial location of the industrial facilities and various pieces of information such as industry type, partial list of chemicals, possible hazards, and/or other site characteristics. Sites varied in proximity to the community and geographic features. A limitation during the testing was that some of the chemical, industry, and hazard prediction was done manually because the analytic programming has not been completed. 


\section{Appendix E: Email from the Army Intelligence Center}

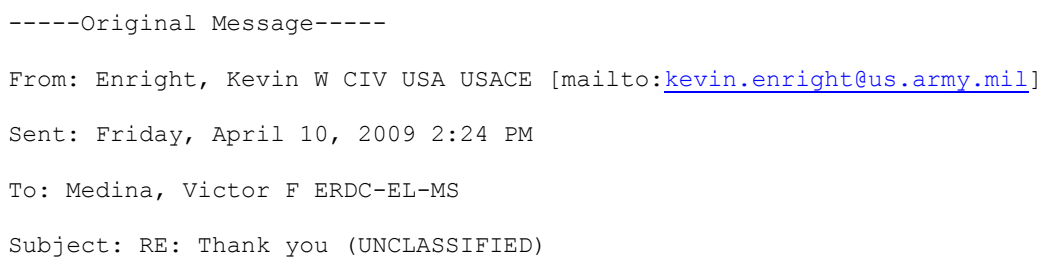


Modernized Integrated Database (MIDB) is a Department of Defense

Intelligence Information System (DODIIS) Intelligence Mission Application, serves as the primary repository for data production and dissemination of military intelligence involving worldwide orders of battle, facilities, command and control networks, targeting, battle damage assessments, and other related information required for strategic assessments and national policy decision-making. 


\section{Appendix F: White Paper for Modification of TIC-Master for Use by installations}




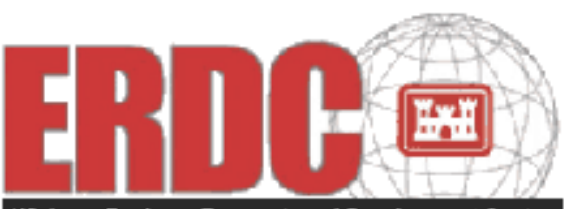

US Amy Enathoor Rusoaren and Lovelopmont Center

November 2010

\section{Installation Chemical Planning, Mapping and Response Tool}

Previously, ERDC developed a GIS linked database tool that allows military planners to predict toxic industrial chemicals from industrial facilities during the Intelligence Preparation of the Battlefield (IPB). Modifying this tool for application at military facilities could have tremendous value in planning and responding to chemical incidents.

TIC Database/GIS Platform Integration:

A database has been built that currently contains over 2,000 TICs, primarily from the International Task Force 40 and EPA Toxic Release Inventory (TRI). The database includes physical properties, hazard assessment information, and environmental effects and remediation information. A data model has been built to relate these chemicals and industrial types as listed as 6 digit codes from the North American Industrial Classification System (NAICS). Data to relate the chemicals to the industries was queried from the TRI database. Analysis of the data allows

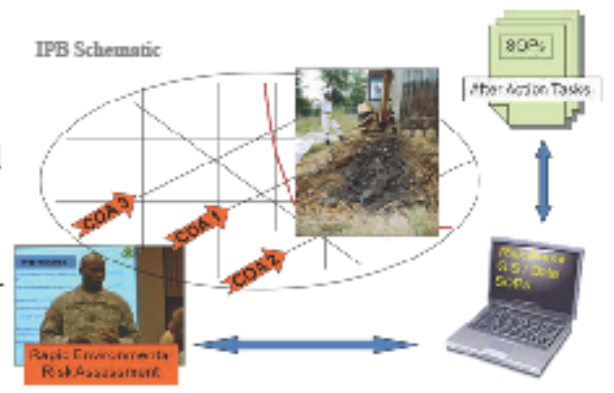
for a crude estimation of the probability to encounter specific TICs at a particular industrial facility. A custom GIS standards based platform has been designed to specifically map industrial facilities within a designated region. The integration of the database and the GIS platform allows relevant information about the chemical (s) to be queried from the TIC database for a specific site and the mapping of areas of highest hazard probability. This technology offers a physical layout of industrial chemical hazards that can be the basis for a more localized mapping of chemical hazards associated with buildings and facilities at military installations.

Test 5: Comparison of Two Facilities

- Evaluated polential operation against 2 facilities, determined which

presented least risk.

- Determined community risk ischools, population etc.l

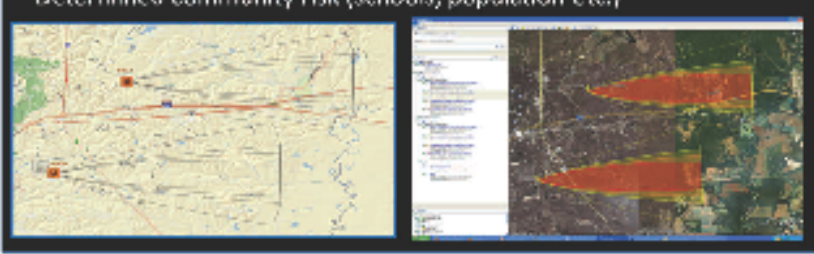

-Successfully determined industries from partial I st of chemicals

Developmental Testing:

A series of test scenarios were created by a semi-independent team to test the predictive aspects of the integrated geospatial system along with the TIC database. The tests were conducting using actual industrial facilities in the Vickburg Mississippi area using EPA Tier 2 data. There were five scenarios given to the test team that represented a broad spectrum of industries and hazardous situations. The industrial process, TIC release mechanism (depending on military action) and atmospheric conditions changed with each. Each scenario provided the geospatial location of the industrial facilities and various pieces of information such as industry type, partial list of chemicals, possible hazards, and/or other site characteristics. Sites varied in proximity to the community and geographic features. A limitation during the testing was that some of the chemical, industry, and hazard prediction was done manually because the analytic programming has not been completed. 


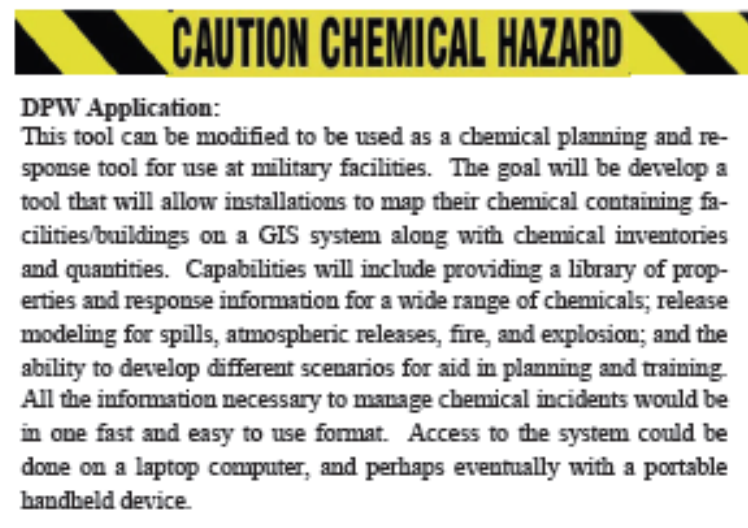

Steps to Developing Installation Chemical Planning, Mapping and Response Tool:

1. Implement previously suggested improvements to chemical database.

2. Obtain detailed information from targeted installation: buildings, inventories, contacts, procedures, etc.

(DANGER)

HAZARDOUS

3. Incorporate installation information into tool database including spill management documents and methodology. Complete hazard information in chemiCHEvicals cal database to adequately cover inventory chemicals.

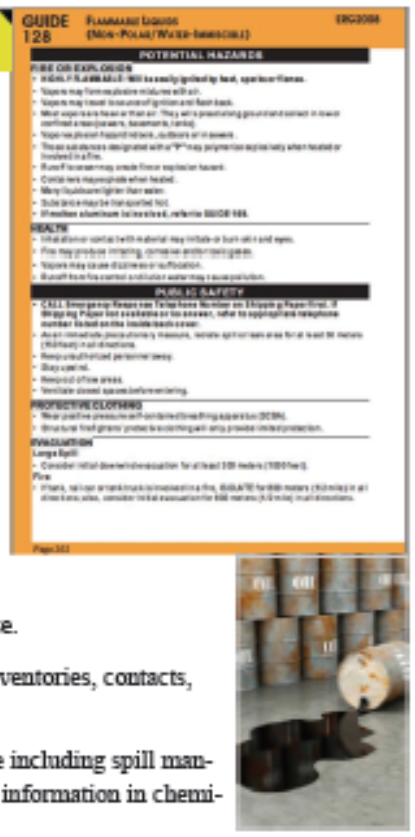

4. Focus GIS system on installation details including terrain t Inprovod respoese to cbemical inci- and special locations of interest.

dants by beving critical chemical

information in an $025 y$ to aso for- 5 . Incorporate inproved release modeling program in place of mat.

Aloha and include liquid/surface release modeling capability.

t The ability to use the tool to dervolop 6. Develop user interface program for intuitive easy-todifferont scenarios will sid in plan- use access to GIS system, database, and methodology for ung wed training spill management.

t Simplo computing requirements

t Elimieste the budcopy roforencen and guesswork

7. Test tool for functionality and applicability.
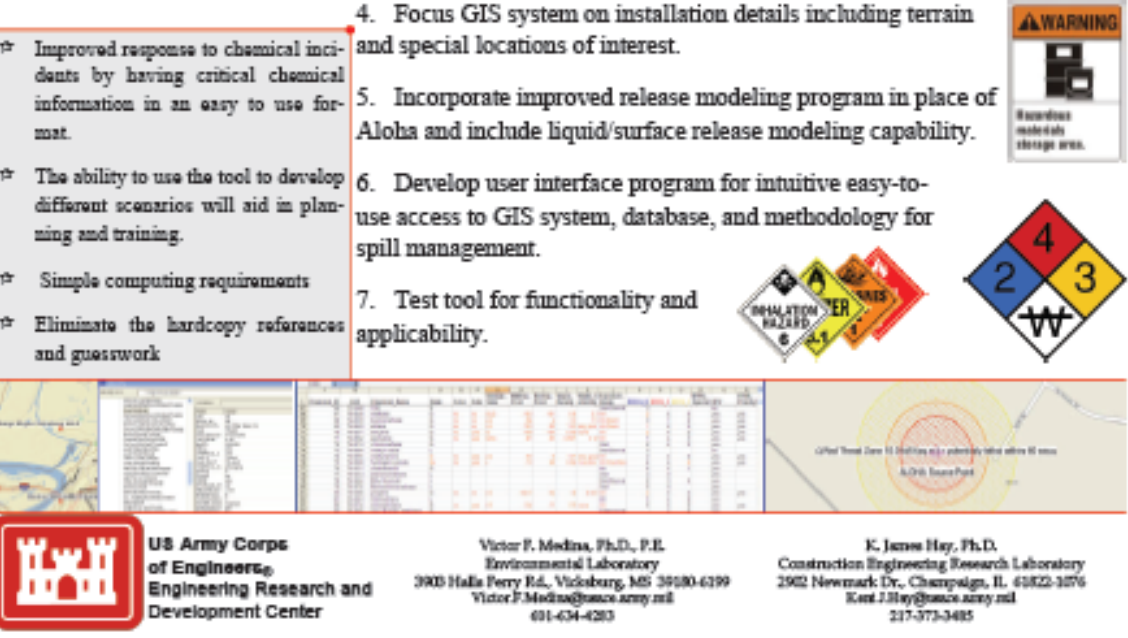


\section{Appendix G: Endorsement from MANSCEN}




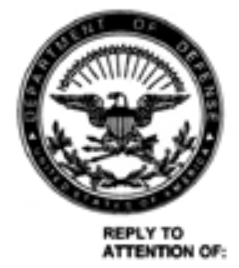

DEPARTMENT OF THE ARMY

U.S. ARMY MANEUVER SUPPORT CENTER AND FORT LEONARD WOOD

320 MANSCEN LOOP STE 316

FORT LEONARD WOOD, MISSOURI 65473-8929

ATZT-CDB

JUN 262009

\section{MEMORANDUM FOR DIRECTOR, U.S. ARMY ENGINEER RESEARCH \& DEVELOPMENT CENTER, 3909 HALLS FERRY ROAD, VICKSBURG, MS 39180-6199}

SUBJECT: Endorsement of Proposed Environmental Intelligence Preparation of the Battlefield Work Package

1. The U.S. Army Maneuver Support Center endorses the proposed work package titled Environmental Intelligence Preparation of the Battlefield.

2. This effort supports the following Warfighter Outcomes:

a. Detection and Identification of Toxic Industrial Chemicals and Material (TIC/TIM)

b. Detect, identify, and neutralize CBRN/TIM agent dispersal modes (sprayers, CBRN-

filled IEDs, bomblets, etc) at standoff

c. Computer-aided Analysis and Reasoning

d. Geospatial Data Management

3. This project is developing a database of significant Toxic Industrial Chemicals, including their important physical and chemical properties, and including information on their environmental transport, fate, and remediation approaches. This database will allow users to access these chemicals if the industrial processes are known and, conversely, estimate industries based on identified chemicals. This is being linked to a GIS system that will allow mapping of the industrial sites and allow plume modeling to estimate effects. A successful product generated from the project could aid in identifying environmental hazards associated with operational planning and in the evaluation of environmental problems associated with overseas bases. 
ATZT-CDB

SUBJECT: Endorsement of Proposed Environmental Intelligence Preparation of the Battlefield Work Package

4. The MANSCEN POC is Dennis Hutchinson, dennis.hutchinson@us.army.mil, commercial (573) 563-5253, DSN 676.

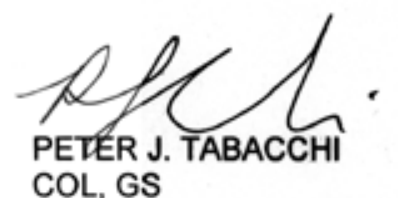
Director, Capability Development and Integration Directorate




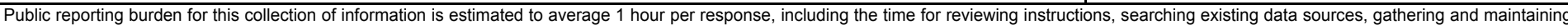

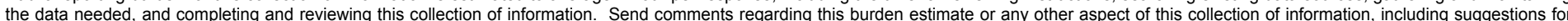

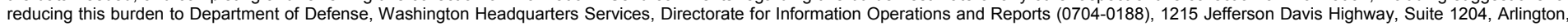

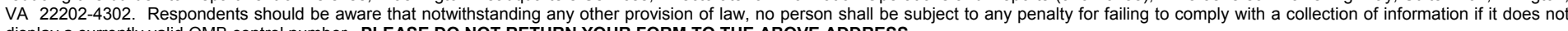
display a currently valid OMB control number. PLEASE DO NOT RETURN YOUR FORM TO THE ABOVE ADDRESS.

\begin{tabular}{c|c}
$\begin{array}{l}\text { 1. REPORT DATE (DD-MM-YYYY) } \\
\text { April } 2012\end{array}$ & $\begin{array}{c}\text { 2. REPORT TYPE } \\
\text { Final Report }\end{array}$ \\
\hline
\end{tabular}

\section{TITLE AND SUBTITLE}

Environmental Assessment During Intelligence Preparation of the Battlefield (IPB) Evaluation of the TIC-Master Computer Program

\section{AUTHOR(S)}

Victor F. Medina, Dennis L. Brandon, K. James Hay, Dhiren S. Khona, Heather K. Smith, and Agnes B. Morrow

\section{DATES COVERED (From - To)}

5a. CONTRACT NUMBER

\section{5b. GRANT NUMBER}

5c. PROGRAM ELEMENT NUMBER

5d. PROJECT NUMBER

5e. TASK NUMBER

5f. WORK UNIT NUMBER

8. PERFORMING ORGANIZATION REPORT NUMBER

U.S. Army Engineer Research and Development Center

3909 Halls Ferry Road, Vicksburg, MS 39180-6199;

ERDC TR-12-5

Construction Engineering Research Laboratory

2902 Newmark Ave., Champaign, IL 61826-9005;

Army Geospatial Center

7701 Telegrapn Rd., Alexandria, VA 22315-3864

9. SPONSORING / MONITORING AGENCY NAME(S) AND ADDRESS(ES)

U.S. Army Corps of Engineers

Washington, D.C. 20314-1000

10. SPONSOR/MONITOR'S ACRONYM(S)

11. SPONSOR/MONITOR'S REPORT NUMBER(S)

\section{DISTRIBUTION / AVAILABILITY STATEMENT}

Approved for public release; distribution is unlimited.

\section{SUPPLEMENTARY NOTES}

\section{ABSTRACT}

The Army needs to manage threats from toxic industrial chemicals (TICs) during the Intelligence Preparation of the Battlefield (IPB) Process. This work unit involved developing a database of TICs that could pose environmental/operational risk, creating an industry/chemical crosswalk, linking the chemical database to a GIS component, applying and evaluating the system in five hypothetical scenarios. The resultant program, which was christened "TIC-Master," has the capability to enhance urban theater of operations analysis to identify TIC/toxic industrial material (TIM) affiliated activities within the battlefield operational footprint and evaluate consequence management alternatives for decision support during the IPB process. TIC-Master was tested in a series of demonstrations. Facilities in the Jackson/Vicksburg MS area were used to test the program. Actual chemical data were obtained for several facilities by securing Tier 2 EPA (Environmental Protection Agency) reports for the facilities of interest. A test team was assembled to develop scenarios based on the industries represented by the facilities. TIC-Master was then used to address the scenarios. Although some deficiencies were identified, the program was successful at encompassing the chemical risk presented by the TICs found at the facilities. Plume modeling was used to assess release scenarios. Subsequent briefings of the program were conducted with potential users at the Army Maneuver Support Center of Excellence (MSCoE) and the Army Intelligency Center (AIC). Constructive suggestions were received and are presented in this document. Overall, the potential users agree that the TIC-Master could be a valuable tool for the Army and other organizations. Ultimately, populating tactical decision-making tools with critical information leads to a greater knowledge of the battlefield and increases survivability.

15. SUBJECT TERMS

Chemical database
Intelligence Preparation of the Battlefield (IPB)

TIC-Master computer program

Toxic industrial chemicals (TICs)

16. SECURITY CLASSIFICATION OF:

a. REPORT

UNCLASSIFIED

b. ABSTRACT
UNCLASSIFIED

c. THIS PAGE

UNCLASSIFIED

\begin{tabular}{l|c|}
$\begin{array}{l}\text { 17. LIMITATION } \\
\text { OF ABSTRACT }\end{array}$ & $\begin{array}{c}\text { 18. NUMBER } \\
\text { OF PAGES }\end{array}$ \\
\cline { 2 - 2 } & 267 \\
\end{tabular}

19a. NAME OF RESPONSIBLE PERSON

19b. TELEPHONE NUMBER (include area code) 